DOE/EIA-0185(97)

Distribution Category UC-950

\title{
Costs and Indices for \\ Domestic Oil and Gas Field Equipment and Production Operations
}

\section{Through 1997}

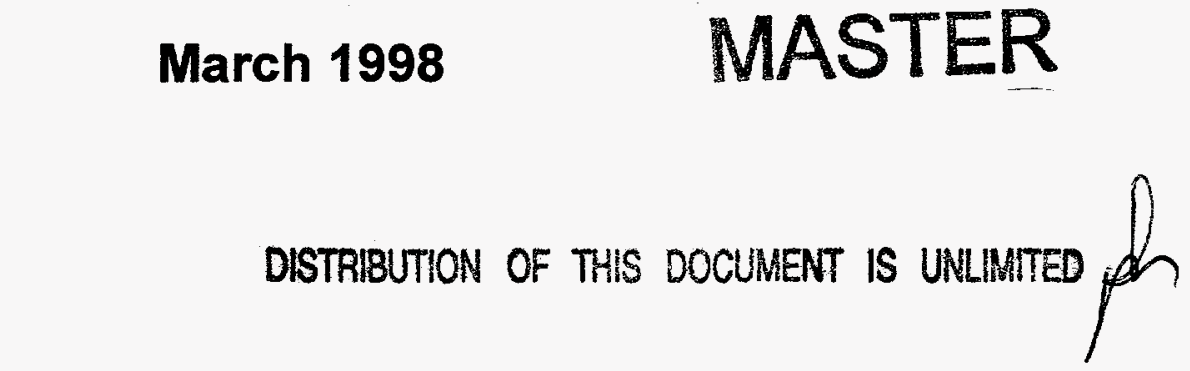

Energy Information Administration

Office of Oil and Gas

U.S. Department of Energy

Washington, DC 20585

This report was prepared by the Energy Information Administration, the independent statistical and analytical agency within the U.S. Department of Energy. The information contained herein should be attributed to the Energy Information Administration and should not be construed as advocating or reflecting any policy position of the Department of Energy or any other organization. 


\section{DISCLAIMER}

This report was prepared as an account of work sponsored by an agency of the United States Government. Neither the United States Government nor any agency thereof, nor any of their employees, makes any warranty, express or implied, or assumes any legal liability or responsibility for the accuracy, completeness, or usefulness of any information, apparatus, product, or process disclosed, or represents that its use would not infringe privately owned rights. Reference herein to any specific commercial product, process, or service by trade name, trademark, manufacturer, or otherwise does not necessarily constitute or imply its endorsement, recommendation, or favoring by the United States Government or any agency thereof. The views and opinions of authors expressed herein do not necessarily state or reflect those of the United States Government or any agency thereof. 


\section{DISCLAIMER}

Portions of this document may be illegible electronic image products. Images are produced from the best available original document. 


\section{Preface}

This is the Energy Information Administration's (EIA's) fifteenth report in the series on domestic costs and indices for oil and gas field equipment and production operations. The purpose of the series is to provide a continuing means of gauging changes in the oil and gas producing industry's costs. The cost data presented in this report are used by government agencies, the academic community, and the oil and gas industry. EIA gratefully acknowledges the cooperation received from personnel of service, supply, and production companies throughout the United States, without which this study would not have been possible. General information about this publication may be obtained from John Wood (214/720-6150), Director of the Reserves and Production Division. Specific information regarding the preparation or contents of this publication may be obtained from Ralph Russell (214/720-6196, rrussell@eia.doe.gov) or Velton Funk (214/720-6171, vfunk@eia.doe.gov), both of whom are petroleum engineers in EIA's Dallas Field Office (fax: 214/720-6155).

All of the tables which appear in this report are available in machine-readable formats, i.e., Lotus 123 or Excel 5.0. Call Ralph Russell at 214/720-6196 or visit the ElAweb site at http//www.eia.doe.gov (press Natural Gas in the Fuel Groups, then Other, then select the appropriate self-extracting file for downloading) 


\section{Contents}

Page

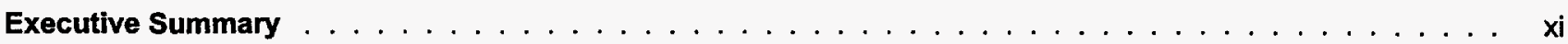

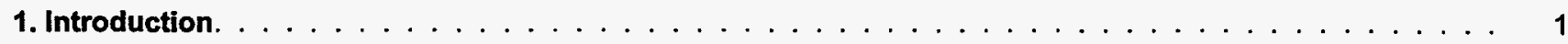

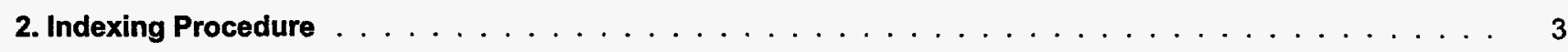

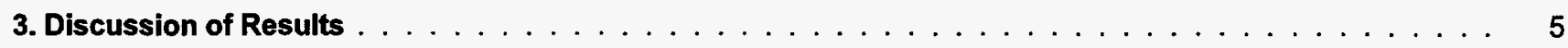

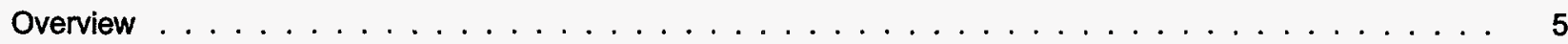

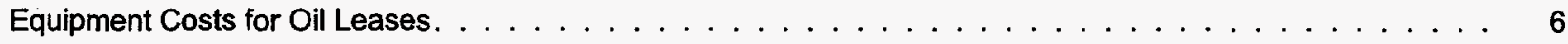

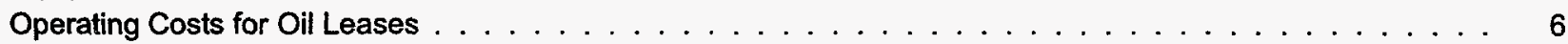

Equipment Costs for Gas Leases . . . . . . . . . . . . . . . . . . . . . . . . . . . . . . 12

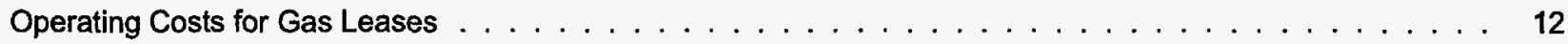

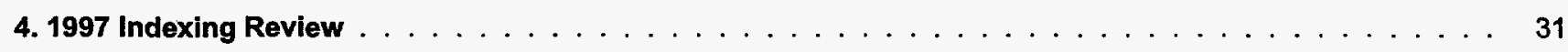

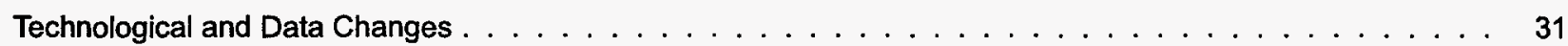

Primary Oil Recovery . . . . . . . . . . . . . . . . . . . . . . . . . . . . . . . . . . . . 31

Secondary Oil Recovery. . . . . . . . . . . . . . . . . . . . . . . . . . . . . . . 32

Offshore Gas and Primary Recovery . . . . . . . . . . . . . . . . . . . . . . . . 32

Gas Recovery . . . . . . . . . . . . . . . . . . . . . . . . . . . . . 32

Appendices

Section I (A throu G)

Costs and Indices for Domestic Oil Field Equipment and Production Operations . . . . . . . . . . . . . 35

Section II ( $H$ through $M)$

Costs and Indices for Domestic Gas Field Equipment and Production Operations ～. . . . . . . . . . . . 69

Section III (N)

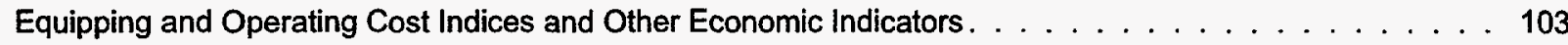

5. Glossary . . . . . . . . . . . . . . . . . . . . . . . . . . . . . . . . . 109 


\section{Tables}

1. Summary of Lease Equipment Costs and Composite Indices for Primary Oil Recovery Operations

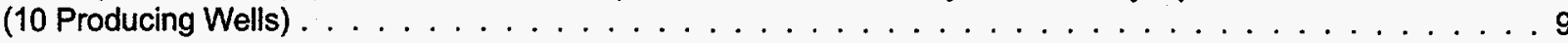

2. Summary of Additional Costs and Composite Indices for Lease Equipment and Injection Wells

in West Texas for Secondary Oil Recovery Operations (10 Producing and 11 Injection Wells) . . . . . . . . . . 11

3. Summary of Direct Annual Operating Costs and Composite Indices for Primary Oil Recovery

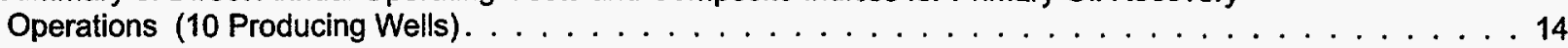

4. Summary of Direct Annual Operating Costs and Composite Indices for Secondary Oil Recovery

Operations in West Texas (10 Producing and 11 Injection Wells). . . . . . . . . . . . . . . . . . 16

5. Summary of Direct Annual Operating Costs and Composite Indices per Platform - Gulf of Mexico $(10,500$-Foot True Vertical Depth Wells) . . . . . . . . . . . . . . . . . . . . . . . . . . . 16

6. Average Equipment Costs and Indices for Gas Leases Aggregated for All Depths, Regions, and Production Rates (One Producing Well) . . . . . . . . . . . . . . . . . . . . . . 19

7. Summary of Gas Lease Equipment Costs and Composite Indices for One Well Producing

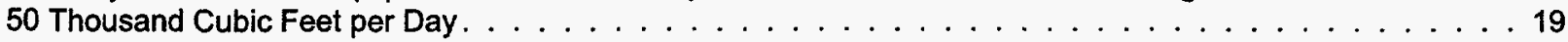

8. Summary of Gas Lease Equipment Costs and Composite Indices for One Well Producing

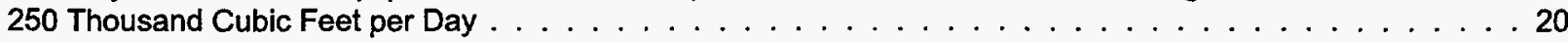

9. Summary of Gas Lease Equipment Costs and Composite Indices for One Well Producing

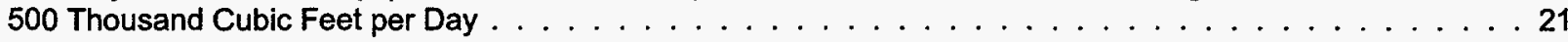

10. Summary of Gas Lease Equipment Costs and Composite Indices for One Well Producing 1 Million Cubic Feet per Day . . . . . . . . . . . . . . . . . . . . . . . 22

11. Summary of Gas Lease Equipment Costs and Composite Indices for One Well Producing 5 Million Cubic Feet per Day . . . . . . . . . . . . . . . . . . . . . . . . . . . 22

12. Summary of Gas Lease Equipment Costs and Composite Indices for One Well Producing 10 Million Cubic Feet per Day. . . . . . . . . . . . . . . . . . . . . . . . . . 23

13. Summary of Aggregate Average Gas Lease Equipment Costs by Depth (1992-1995) . . . . . . . . . . . . . . . 23

14. Average Operating Costs and Indices for Gas Leases Aggregated for All Depths, Regions, and Production Rates (One Producing Well) . . . . . . . . . . . . . . . . . . . . . . . . . . . . 24

15. Summary of Gas Lease Operating Costs and Composite Indicesfor One Well Producing 50 Thousand Cubic Feet per Day. . . . . . . . . . . . . . . . . . . . . . . . . . . . 24

16. Summary of Gas Lease Operating Costs and Composite Indicesfor One Well Producing 250 Thousand Cubic Feet per Day . . . . . . . . . . . . . . . . . . . . . . . . . 25

17. Summary of Gas Lease Operating Costs and Composite Indices for One Well Producing

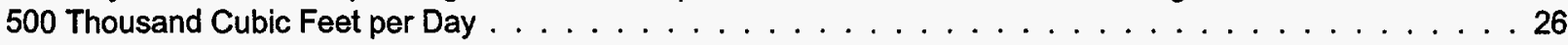

18. Summary of Gas Lease Operating Costs and Composite Indices for One Well Producing 1 Million Cubic Feet per Day . . . . . . . . . . . . . . . . . . . . . . . . . . . . 27

19. Summary of Gas Lease Operating Costs and Composite Indices for One Well Producing 5 Million Cubic Feet per Day . . . . . . . . . . . . . . . . . . . . . . . . . . 28

20. Summary of Gas Lease Operating Costs and Composite Indices for One Well Producing 10 Million Cubic Feet per Day. . . . . . . . . . . . . . . . . . . . . . . . 28

21. Summary of Aggregate Average Gas Lease Operating Costs by Depth . . . . . . . . . . . . . . . . . . . . . 28

22. Type of Artificial Lift and Prime Mover Used for Each Depth andRegion . . . . . . . . . . . . . . . . . . . 31 


\section{Figures}

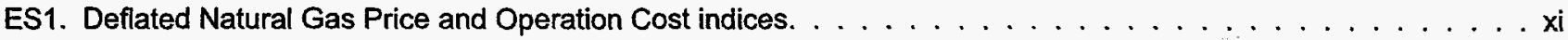

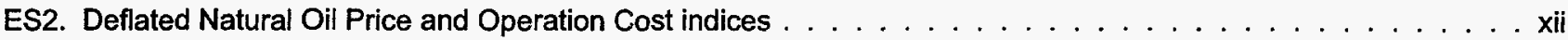

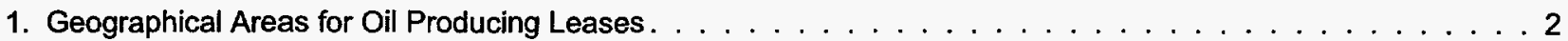

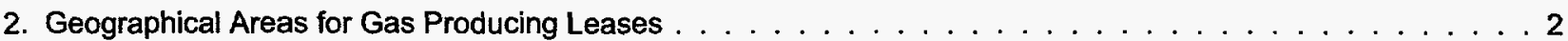

3. Aggregate Average Costs for Primary Oil Recovery, 1976-1995

(Operations and Equipment, With and Without Tubing Costs) $\ldots \ldots \ldots \ldots \ldots$

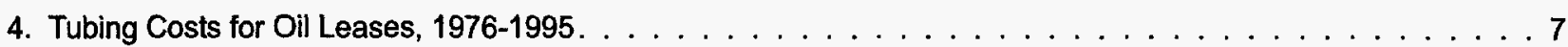

5. Non-Tubing Costs for Oil Leases, $1976-1995 \ldots \ldots \ldots \ldots \ldots \ldots \ldots$

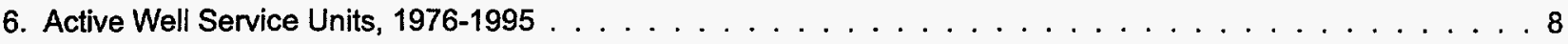

7. Aggregate Average Costs for Gas Recovery, 1976-1995 (Equipment and Operation Cost) . . . . . . . . . . 8

8. Aggregate Average Lease Equipment Costs for Primary Oil Recovery, 1992-1995

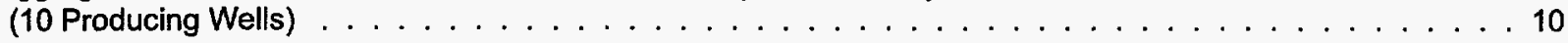

9. Additional Cost of Lease Equipment for Secondary Oil Recovery in West Texas, 1992-1995

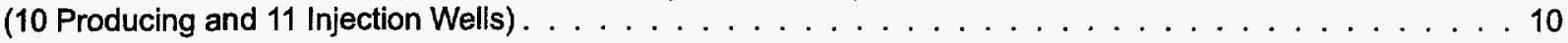

10. Aggregate Annual Operating Costs for Primary Oil Recovery Operations, 1992-1995 . . . . . . . . . . 15

11. Annual Operating Costs for Secondary Oil Recovery in West Texas, 1992-1995

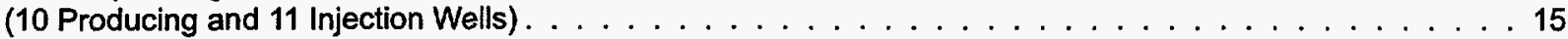

12. Fuel, Power, and Water Cost Indices for 12,000 -foot Oil Wells in California and the Rocky Mountains . . . . . 17

13. Fuel, Power, and Water Cost Indices for Primary and Secondary Operating Costs

for 4,000 -foot Wells in West Texas . . . . . . . . . . . . . . . . . . . . . . . 17

14. Annual Gas Well Equipment Costs by Depth and Production Rate (1995) . . . . . . . . . . . . . . . . 18

15. Aggregate Average Equipment Costs for a One Well Gas Lease by Production Rate, 1992-1995 . . . . . . . 18

16. Aggregate Average Annual Gas Well Operating Costs for a One-Well Gas Lease

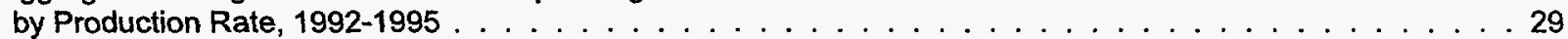

17. Annual Gas Well Operating Costs by Depth and Production Rate, $1995 \ldots \ldots \ldots$

N1. Gross Domestic Product Deflated Producer Price Indices, and Oil and Gas Field

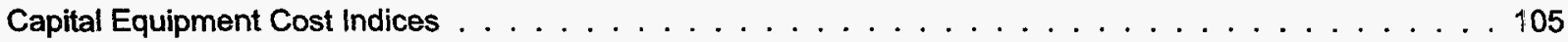

N2. Gross Domestic Product Deflated Operating Cost Indices for Oil and Gas Fields . . . . . . . . . . . . . 105

Appendix A

Lease Equipment Costs and Indices for Primary Oil Production in West Texas

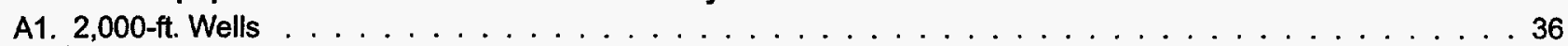

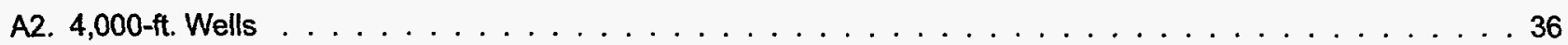

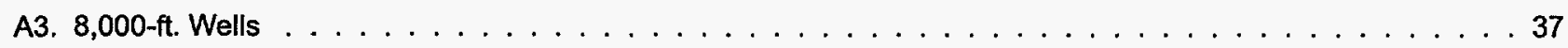

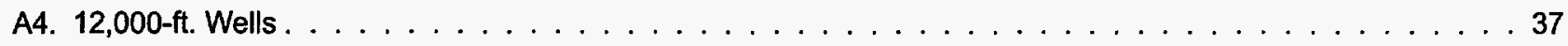

Direct Annual Operating Costs and Indices for Primary Oil Production in West Texas

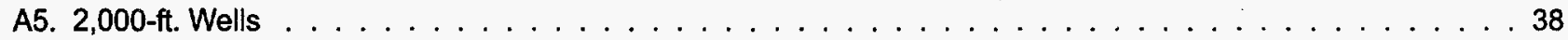

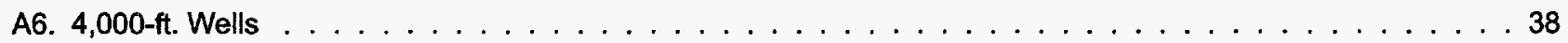

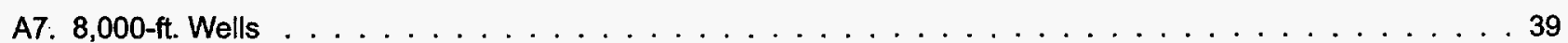

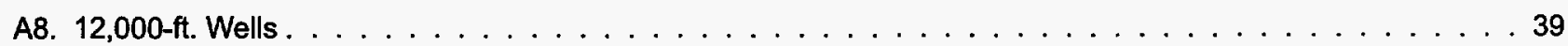

Additional Lease Equipment and Well Costs and Indices for Secondary Oil Production in West Texas

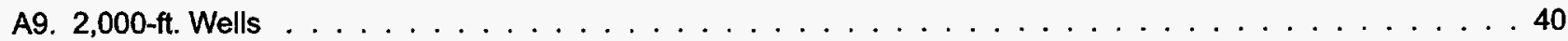

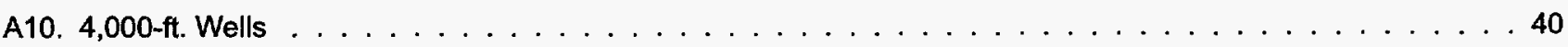

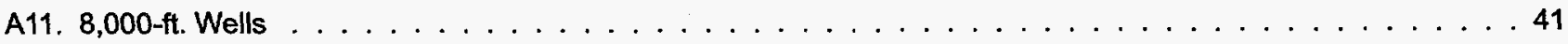


Direct Annual Operating Costs and Indices for Secondary Oil Production in West Texas

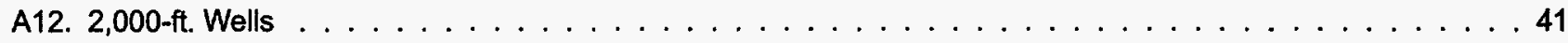

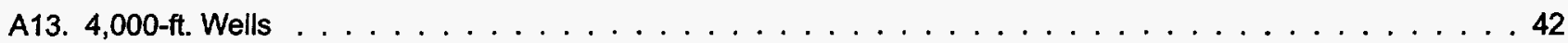

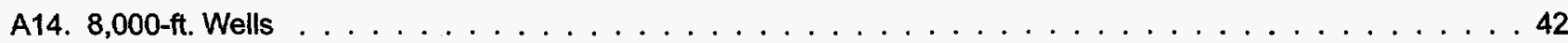

Detailed Equipment List

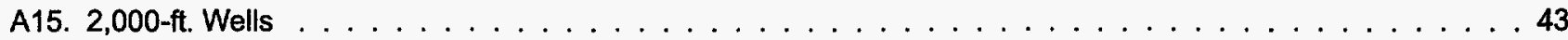

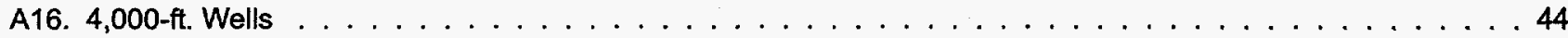

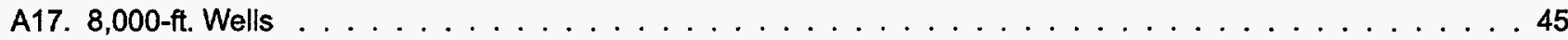

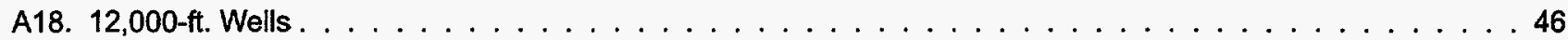

Appendix B

Lease Equipment Costs and Indices for Primary Oil Production in South Texas

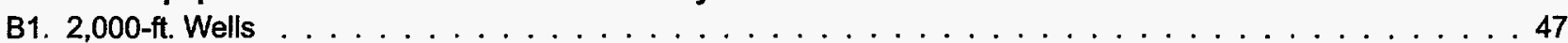

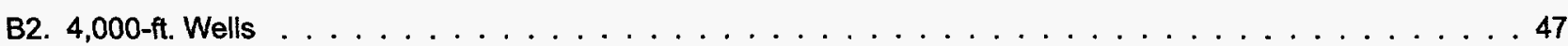

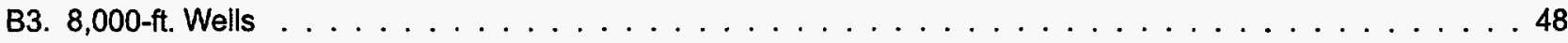

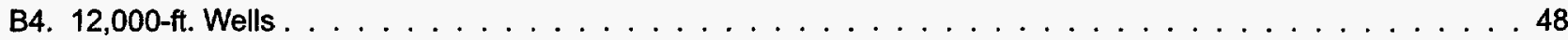

Direct Annual Operating Costs and Indices for Primary Oil Production in South Texas

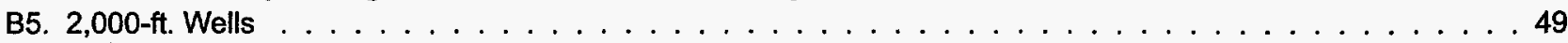

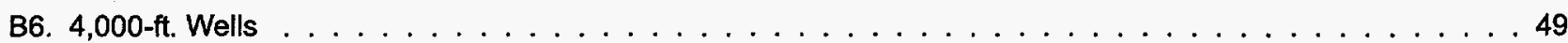

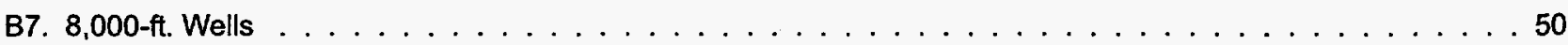

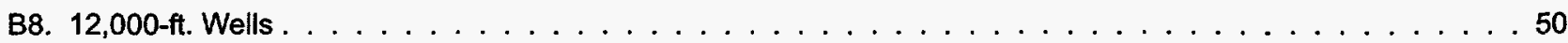

Appendix C

Lease Equipment Costs and Indices for Primary Oil Production in South Louisiana

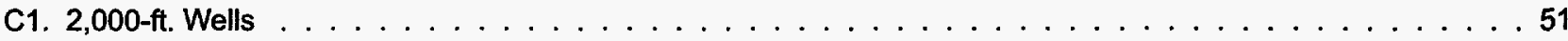

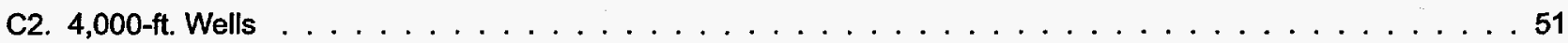

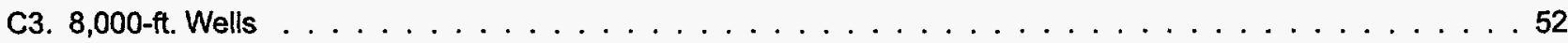

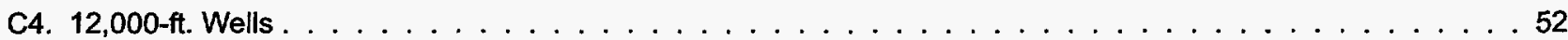

Direct Annual Operating Costs and Indices for Primary OII Production in South Louisiana

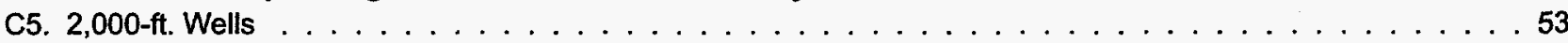

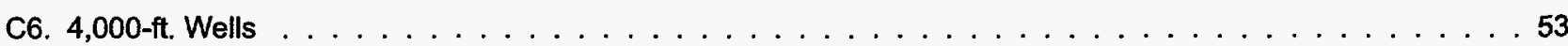

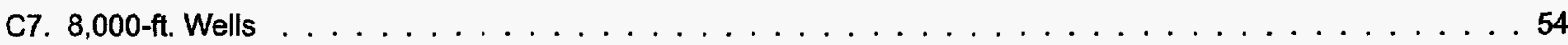

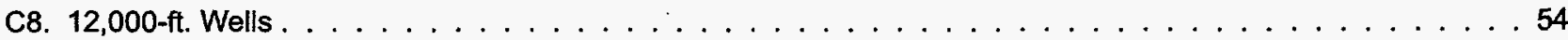

Appendix D

Lease Equipment Costs and Indices for Primary Oil Production in Oklahoma

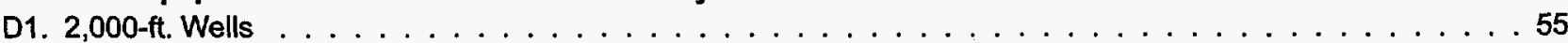

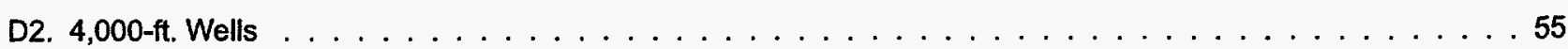

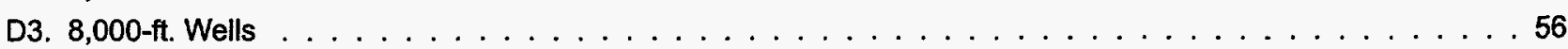

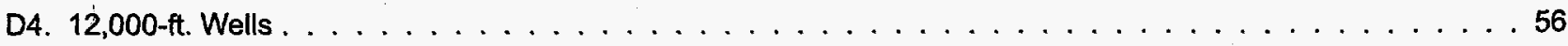

Direct Annual Operating Costs and Indices for Primary Oil Production in Oklahoma

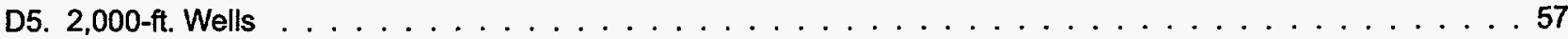

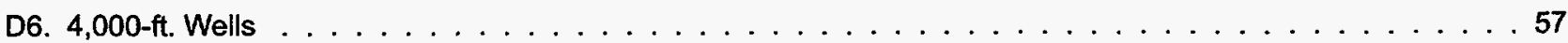

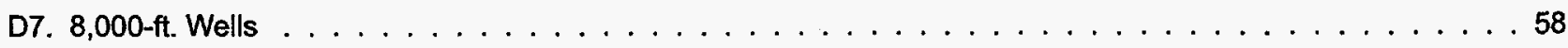

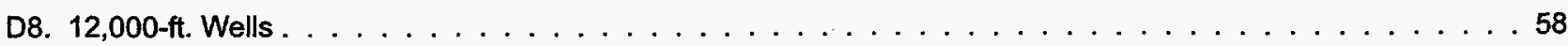


Appendix E

Lease Equipment Costs and Indices for Primary Oil Production in the Rocky Mountains

E1. 2,000-ft. Wells . . . . . . . . . . . . . . . . . . . . . . . . . . . . . . . . . . . . .

E2. 4,000-ft. Wells . . . . . . . . . . . . . . . . . . . . . . . . . . . 59

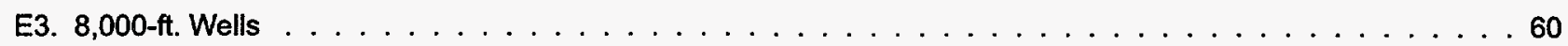

E4. 12,000-ft. Wells. . . . . . . . . . . . . . . . . . . . . . . . . . . . . 60

Direct Annual Operating Costs and Indices for Primary Oil Production in the Rocky Mountains

E5. 2,000-ft. Wells . . . . . . . . . . . . . . . . . . . . . . . . . . 61

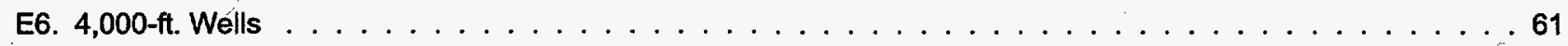

E7. 8,000-ft. Wells . . . . . . . . . . . . . . . . . . . . . . . . . 62

E8. 12,000-ft. Wells . . . . . . . . . . . . . . . . . . . . . . . . . . 62

Appendix F

Lease Equipment Costs and Indices for Primary Oil Production in California

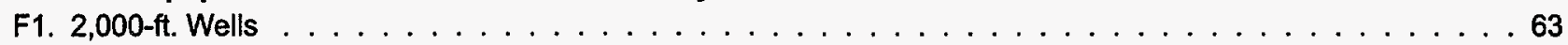

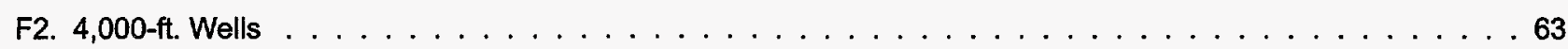

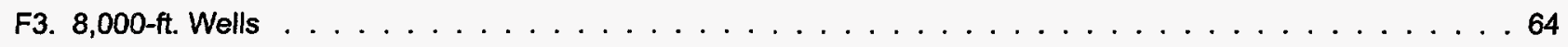

F4. 12,000-ft. Wells . . . . . . . . . . . . . . . . . . . . . . . . . . . . . . . 64

Direct Annual Operating Costs and Indices for Primary Oil Production in California

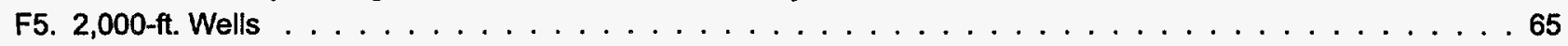

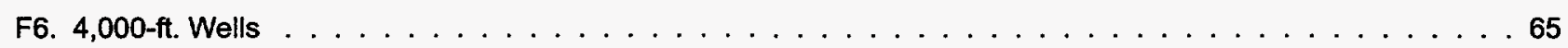

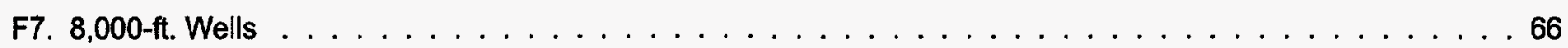

F8. 12,000-ft. Wells . . . . . . . . . . . . . . . . . . . . . . . . . 66

Appendix $\mathbf{G}$

Annual Operating Costs and Indices for Platforms in the Gulf of Mexico

G1. 100-foot Water Depth (12-Slot Platform) . . . . . . . . . . . . . . . . . . . . 67

G2. 300-foot Water Depth (12-Slot Platform) . . . . . . . . . . . . . . . . . . . . 67

G3. 100-foot Water Depth (18-Slot Platform) . . . . . . . . . . . . . . . . . . . . . . . . . 67

G4. 300-foot Water Depth (18-Slot Platform) . . . . . . . . . . . . . . . . . . . . . 68

G5. 600-foot Water depth (18-Slot Platform) . . . . . . . . . . . . . . . . . . . . 68

Appendix H

Lease Equipment Costs and Indices for Gas Production in West Texas

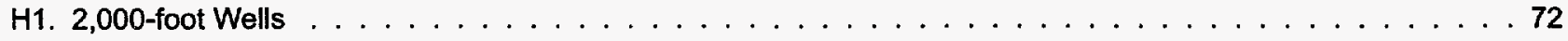

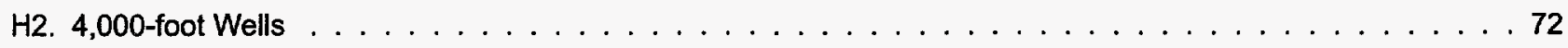

H3. 8,000-foot Wells . . . . . . . . . . . . . . . . . . . . . . . . . . . 73

H4. 12,000-foot Wells . . . . . . . . . . . . . . . . . . . . . . . . . . . 73

H5. 16,000-foot Wells . . . . . . . . . . . . . . . . . . . . . . . . . . 74

Direct Annual Operating Costs and Indices for Gas Production in West Texas

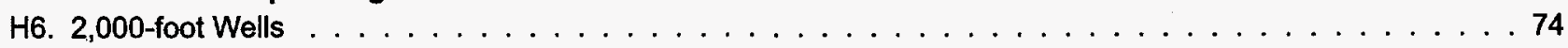

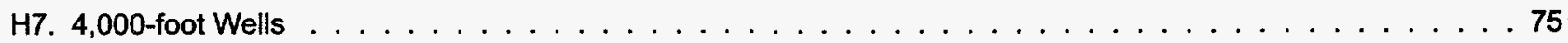

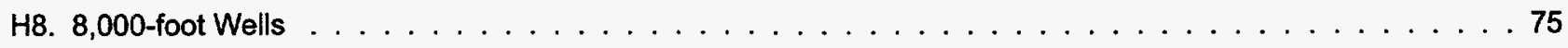

H9. 12,000-foot Wells . . . . . . . . . . . . . . . . . . . . . . . . . . 76

H10. 16,000-foot Wells . . . . . . . . . . . . . . . . . . . . . . . . . . . 76

H11. Detailed Lease Equipment List for 12,000-Foot Gas Wells in West Texas, Producing

1 Million Cubic Feet per Day. . . . . . . . . . . . . . . . . . . . . . . . . . . 77 
Appendix 1

Lease Equipment Costs and Indices for Gas Production in South Texas

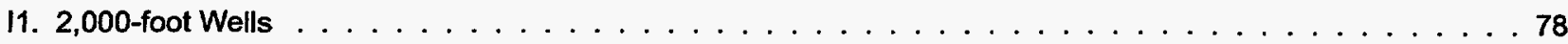

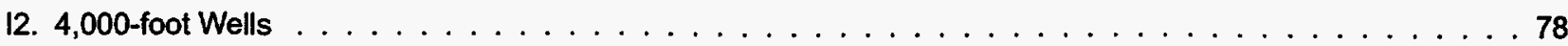

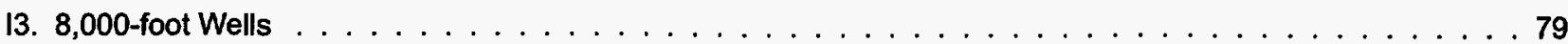

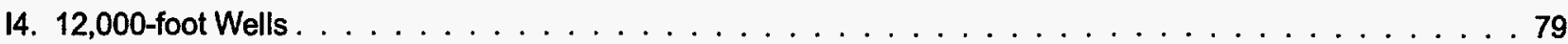

Direct Annual Operating Costs and Indices for Gas Production in South Texas

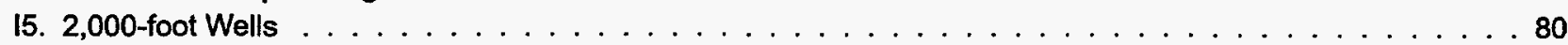

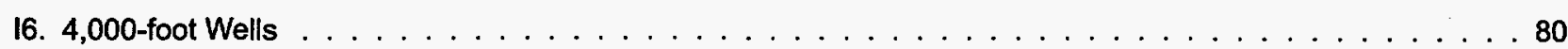

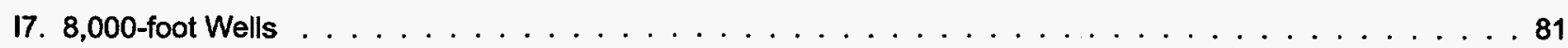

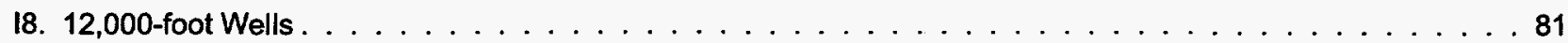

Appendix $\mathbf{J}$

Lease Equipment Costs and Indices for Gas Production in South Louisiana

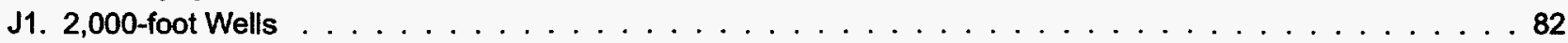

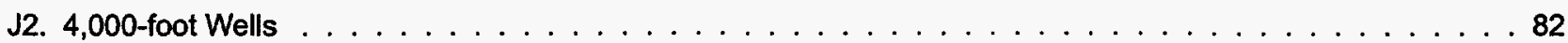

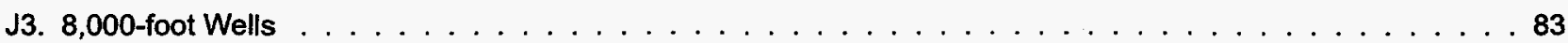

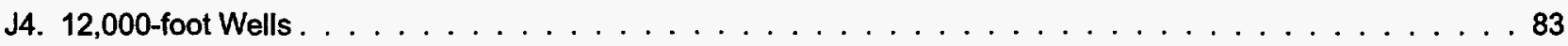

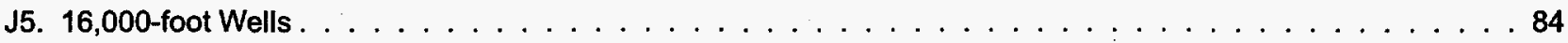

Direct Annual Operating Costs and Indices for Gas Production in South Louisiana

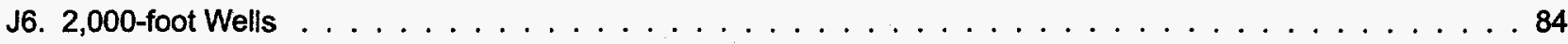

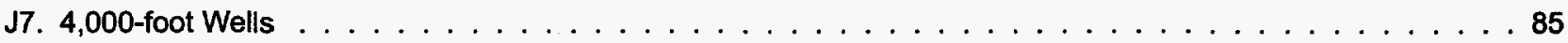

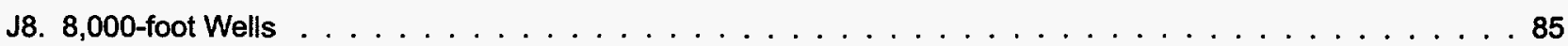

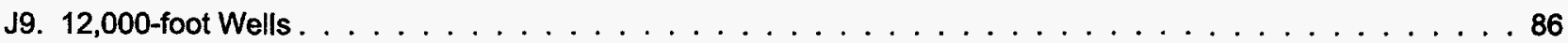

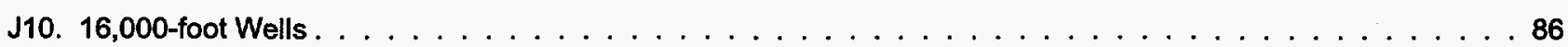

Appendix K

Lease Equipment Costs and Indices for Gas Production in North Louisiana

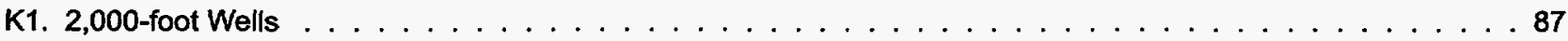

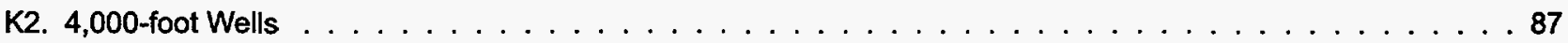

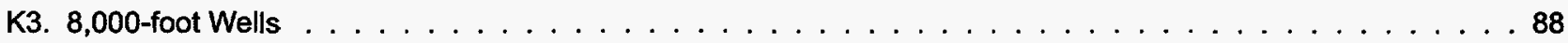

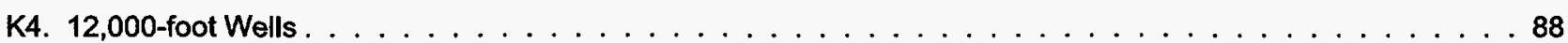

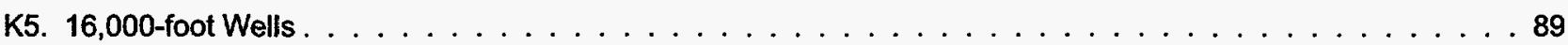

Direct Annual Operating Costs and Indices for Gas Production in North Louisiana

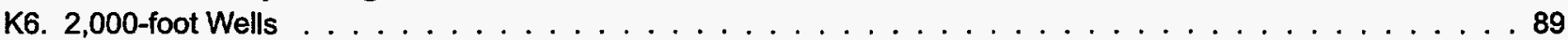

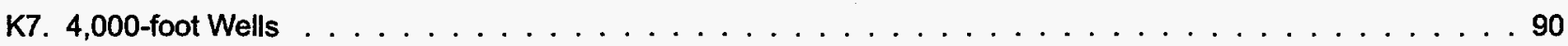

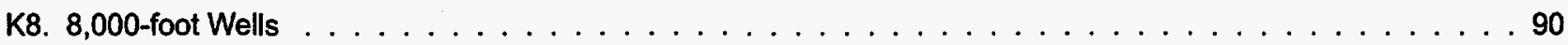

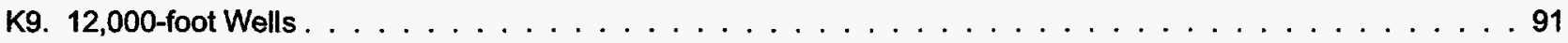

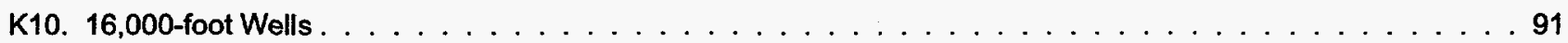

Appendix L

Lease Equipment Costs and Indices for Gas Production in the Mid-Continent

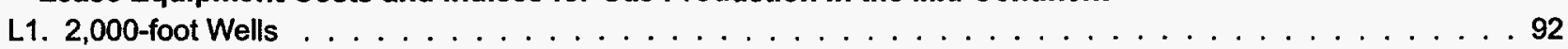

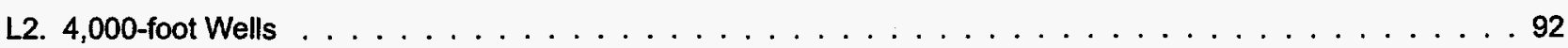

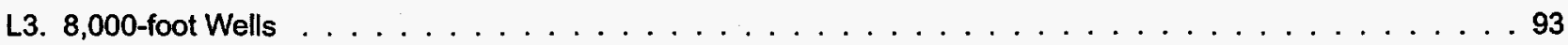

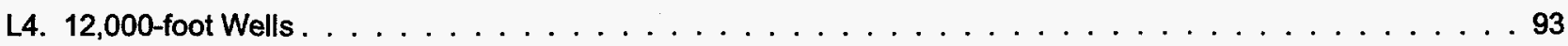

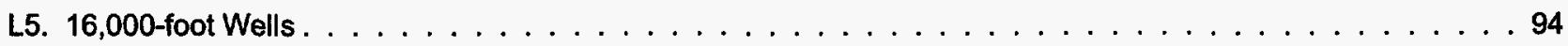


Direct Annual Operating Costs and Indices for Gas Production in the Mid-Continent

L6. 2,000-foot Wells . . . . . . . . . . . . . . . . . . . . . . . . . . . . . . . . . . . . .

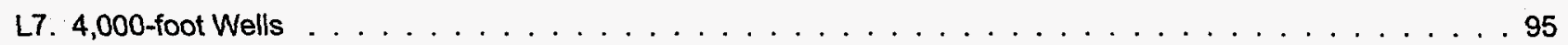

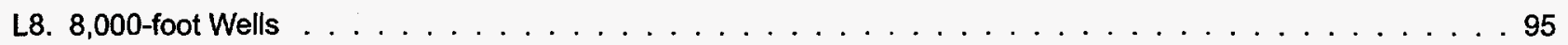

L9. 12,000-foot Wells . . . . . . . . . . . . . . . . . . . . . . . . . . 96

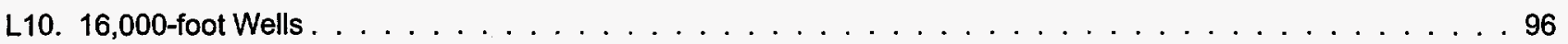

Appendix $M$

Lease Equipment Costs and Indices for Gas Production in the Rocky Mountains

M1. 2,000-foot Wells . . . . . . . . . . . . . . . . . . . . . . . . . . . 97

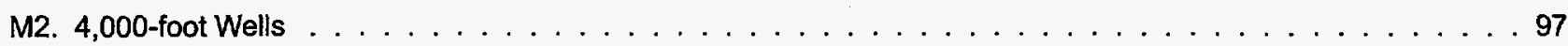

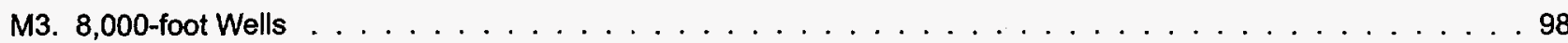

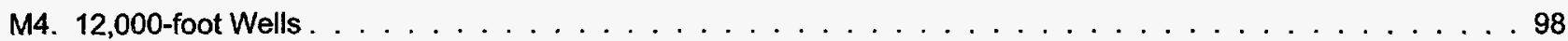

Direct Annual Operating Costs and Indices for Gas Production in the Rocky Mountains

M5. 2,000-foot Wells . . . . . . . . . . . . . . . . . . . . . . . . . . . . 99

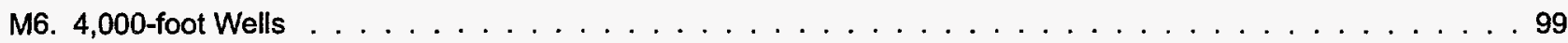

M7. 8,000-foot Wells . . . . . . . . . . . . . . . . . . . . . . . . . . 100

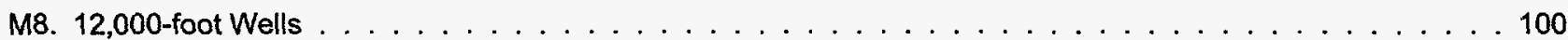

Appendix $\mathbf{N}$

N1. Indices and Gross Domestic Product Deflated Indices of the Aggregated Average Capital Equipment Costs for Oil and Gas Fields and the Producer Price Index (PPI) . . . . . . . . . . . . . . . . . . 104

N2. The Gross Domestic Product Implicit Price Deflator and the Gross Domestic Product Deflated Indices of Operating Costs for Oil and Gas Fields . . . . . . . . . . . . . . . . . . . . . . . . . . . . 104

N3. 


\section{Executive Summary}

This report presents estimated costs and cost indices for domestic oil and natural gas field equipment and production operations for 1994, 1995, 1996, and 1997. The costs of all equipment and services are those in effect during June of each year. The sums (aggregates) of the costs for representative leases by region, depth, and production rate were averaged and indexed. This provides a general measure of the increased or decreased costs from year to year for lease equipment and operations. These general measures do not capture changes in industry-wide costs exactly because of annual variations in the ratio of the total number of oil wells to the total number of gas wells. The detail provided in this report is unavailable elsewhere. The body of this report contains summary tables, and the appendices contain detailed tables.

Price changes for oil and gas, changes in taxes on oil and gas revenues, and environmental factors (compliance costs and lease availability) have a significant impact on the number and cost of oil and gas wells drilled. These changes also impact the cost of oil and gas equipment and production operations.
Oil and gas prices rose from 1976 to the early 1980's, when deflated oil prices peaked at an index of about 260. In 1976, the average price of oil was $\$ 8.19$ per barrel and the average price of gas was $\$ 0.58$ per thousand cubic feet (Mcf). Deflated gas prices, which also rose to an index of about 270 , were at a plateau from 1982 through 1984, before following oil prices downward. The 1995 oil price, after an increase from 1994, represents the second-lowest deflated oil price since 1976. By contrast, deflated gas prices have remained above 1976 prices, and, after heading downward in 1994 and 1995 , rose in 1996 and 1997. Clearly, the price trends reflect fundamental differences between the markets for oil and gas.

Figure ES1, with gas prices and operating costs indexed to 1976 , shows the variations in deflated gas prices and deflated operating costs for gas wells. The greatest difference between the two series was during 1984, and the current downward trend in product prices, coupled with declining operating costs, point to the fact that producer profitability is much more strongly affected by product prices than by increasing operating efficiency.

Gas activity has been spurred in recent years by favorable tax treatment (including tax credits for tight formations gas and

Figure ES1. Deflated Natural Gas Prices and Operating Cost Indices

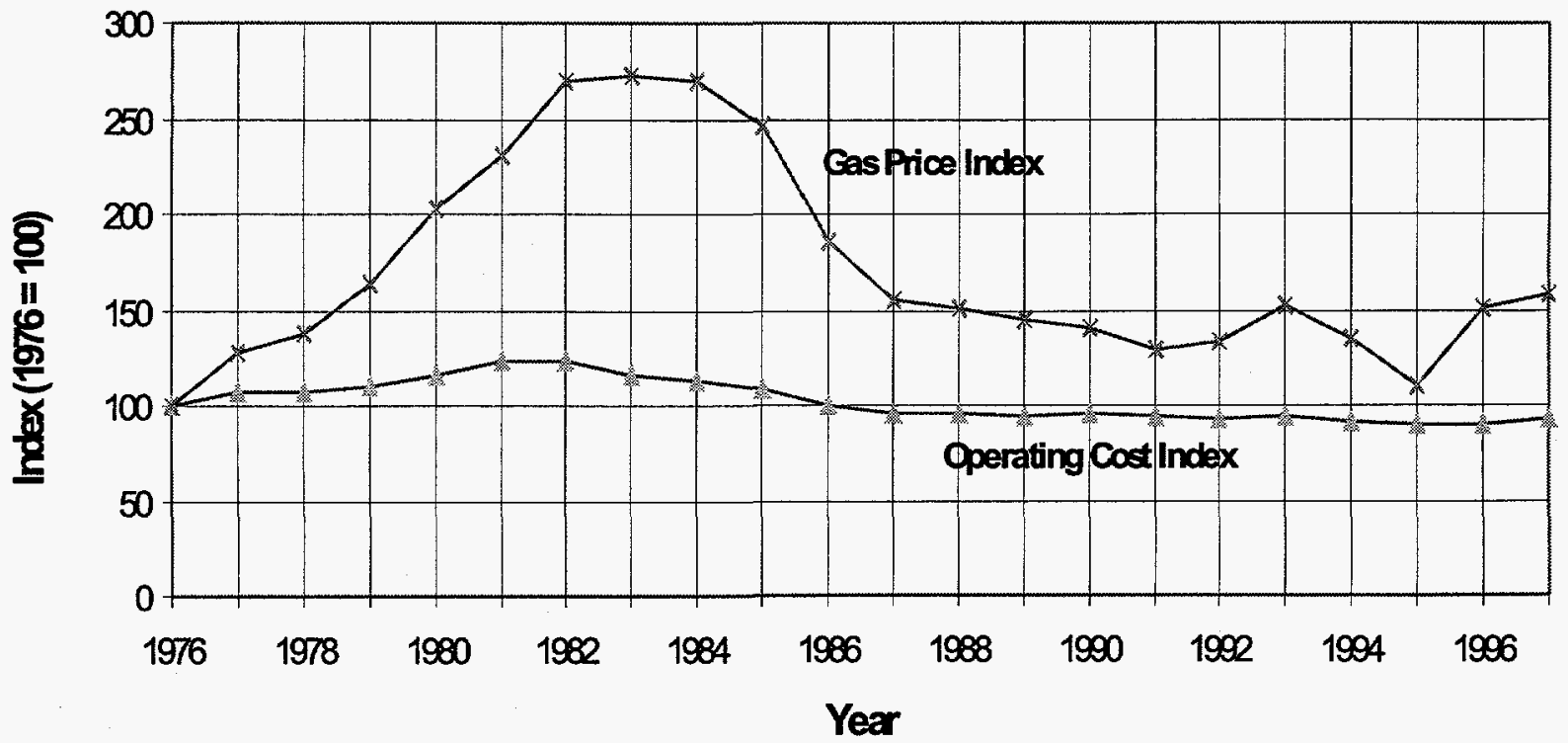

Source: Energy Information Administration, Office of Oil and Gas. 
coalbed methane). Environment-related costs for natural gas operations, generally less than for oil operations, may equal those for oil where coalbed methane leases are concerned as the main factor affecting operating costs in some coalbed methane regions is disposal of substantial amounts of formation water produced with the gas. No data have been collected on the environmental costs for gas wells.

Figure ES2 similarly depicts deflated oil prices and operating costs indexed to 1976. There are two main differences between Figures ES1 and ES2. Gas prices have remained nearly 50 percent higher than those in the 1976 base year, while oil prices have rarely reached 1976 levels after the collapse of 1986 . And, while deflated gas operating costs have remained below 1976 levels since 1986, deflated oil operating costs have never subsided to 1976 levels.

Oil production is an energy-intensive operation, and when fuel prices (natural gas prices) increase, so do oil production costs. Gas production is more labor intensive, with only minor fuel costs. Therefore, high energy prices are a boon to gas producers, and the natural gas-producing industry has fared better than the oil-producing industry for the past decade. The change in gas prices has surpassed the change in gas well operating costs. Oil prices have fallen faster arid farther than oil field operating costs since 1981, narrowirig the profit margin and reducing the amount of internally raised capital available for investment in drilling and production operations.

Useful insights from the data in this report lie primarily in the differences that are presented. The costs for equipment and operations are different in each area, differing between primary and secondary operations and differing between gas and oil operations. Cost trends for some items vary widely from time to time, while others remain unchanged for years at a time. The summary tables in Chapter 3, Discussion of Results, indicate the overall trends while the Appendix tables provide the detail needed by many readers.

Figure ES2. Deflated Oil Price and Operating Cost Indices

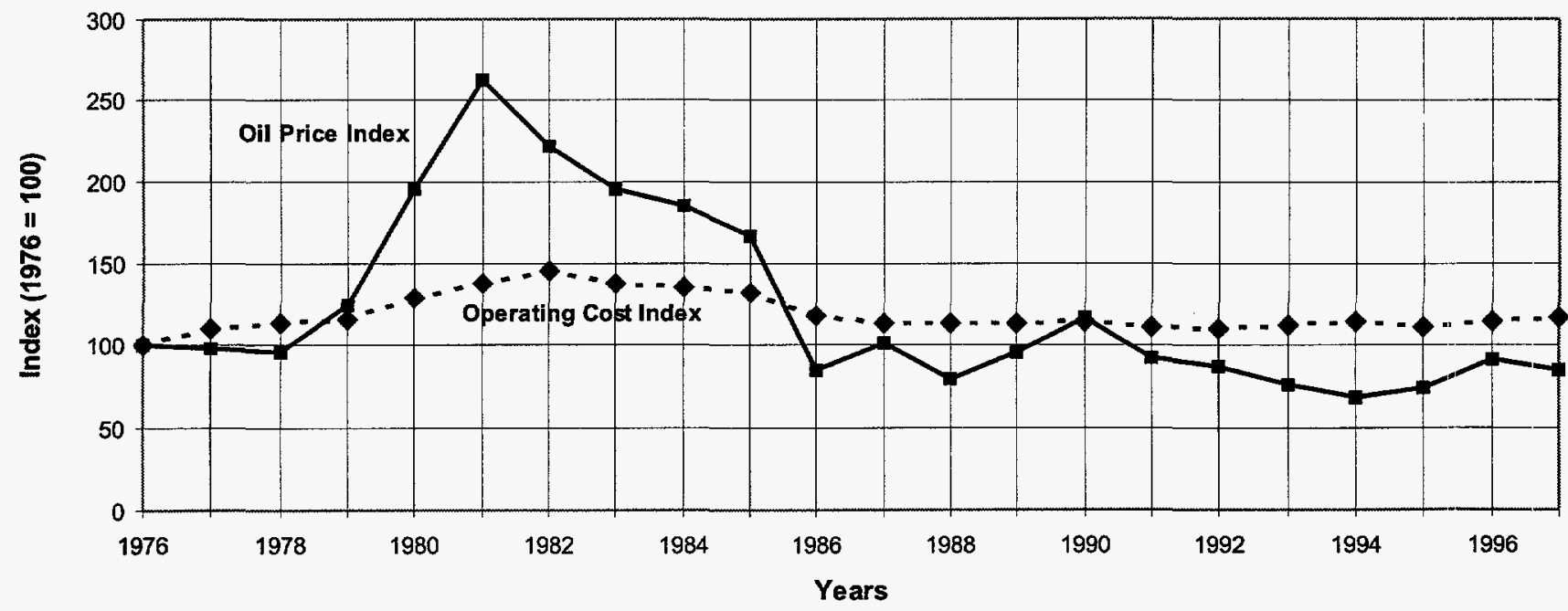

Source: Energy Information Administration, Office of Oil and Gas. 


\section{Introduction}

This report marks the continuation of the EIA series on equipment and operating costs and cost indices for oil and gas leases. In addition to cost comparisons within the petroleum industry, the data reported here are often used to assess the economic effects of specific plans and policies relating to the industry. No other source offers the detail of this report.

Only a few publications contain or have contained data relating to costs in the petroleum industry. For several years, the American Petroleum Institute (API), the Inde pendent Petroleum Association of America (IPAA), and the Mid-Continent Oil and Gas Association have published cost statistics in their Joint Association Survey of the U.S. Oil and Gas Producing Industry. ${ }^{1,2}$ Section I of that publication pertains to drilling costs. Section II (discontinued after 1975) presented total U.S. expenditures for exploration, development, and production.

For several years, the U.S. Bureau of the Census surveyed a group of companies classified by size and published a report titled Annual Survey of Oil and $\mathrm{Gas}^{3}$ in its Current Industrial Report series. These reports contained oil and gas operating cost data for both direct and indirect expenses but were discontinued after 1982 .

Other than EIA's, no series of non-aggregated oil and gas field equipment and operating costs and subsequent indices has been published on a regional basis. Equipment and operating costs vary from region to region because of differences in fuel costs, labor rates, and other variables. (See Figures 1 and 2 for areas of oil and gas production.) Therefore, equipment and operating costs and cost indices are estimated by EIA on a regional basis for both oil and gas fields.

The costs and cost indices provided in this report are for representative lease operations with equipment and operating procedures designed by EIA staff engineers for representative 10-well oil leases producing by artificial lift or 1 flowing well per gas lease. The design criteria took into account the predominant methods of operation in each region. Individual items of equipment were priced by using price lists and by communication with the manufacturer or supplier of the item in each region. Except as mentioned in the Executive Summary and treated in Appendix N, all costs presented in this report are current to their year and are not adjusted for inflation.

Freight costs and installation costs were determined on the basis of regional rates. These costs were summed for each category of equipment. For example, the category listed as "pumping equipment" for a rod pump system includes:

- A pumping unit

- Additional counter-weights

- Crank guards

- Belt guards, V-belts, and sheaves

- Freight costs

- Installation costs.

Conversion of primary oil-producing leases to secondary recovery (waterflood or water injection) involves:

- the drilling and equipping of 11 water injection wells

- the installation of water supply, storage, treatment, high pressure injection equipment, and related piping

- replacement of production facilities with larger equipment.

Costs for gas activities were investigated by determining equipping and operating costs for representative gas leases producing from depths of $2,000,4,000,8,000,12,000$ and 16,000 feet in 6 onshore regions of the lower 48 States. The summary tables contain composite costs and indices for flow rates of $50,250,500,1,000,5,000$, and 10,000 thousand cubic feet (Mcf) of gas per day by depth and region.

Equipment for gas wells does not cover hydrogen sulfide removal, compression, or special equipment for water removal. Tubing is also not included in the equipment list for gas wells.

\footnotetext{
${ }^{1}$ American Petroleum Institute, Independent Petroleum Association of America, Mid-Continent Oil and Gas Association, 1995 Joint Association Survey on Drilling Costs. (Washington, DC, November 1996), American Petroleum Institute.

${ }^{2}$ American Petroleum Institute, Independent Petroleum Association of America, Mid-Continent Oil and Gas Association, Joint Association Survey of the U.S. Oil and Gas Producing Industry, 1974, Section II: Expenditures for Exploration, Development and Production, (Washington, DC, May 1976), American Petroleum Institute.

${ }^{3}$ U.S. Bureau of the Census, Annual Survey of Oil and Gas, 1981, Current Industrial Reports pub. MA-13K (81)-1, (Washington, DC, March 1983).
} 
Figure 1. Geographical Regions for Oil Producing Leases

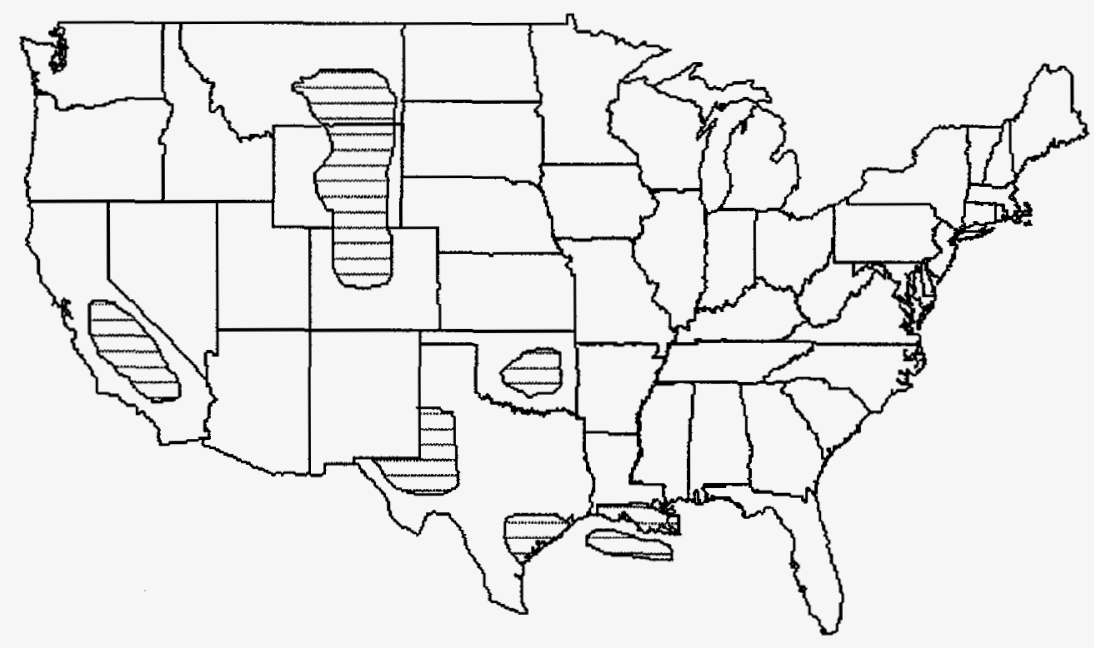

Source: Energy Information Administration, Office of Oil and Gas.

Figure 2. Geographical Regions for Gas Producing Leases

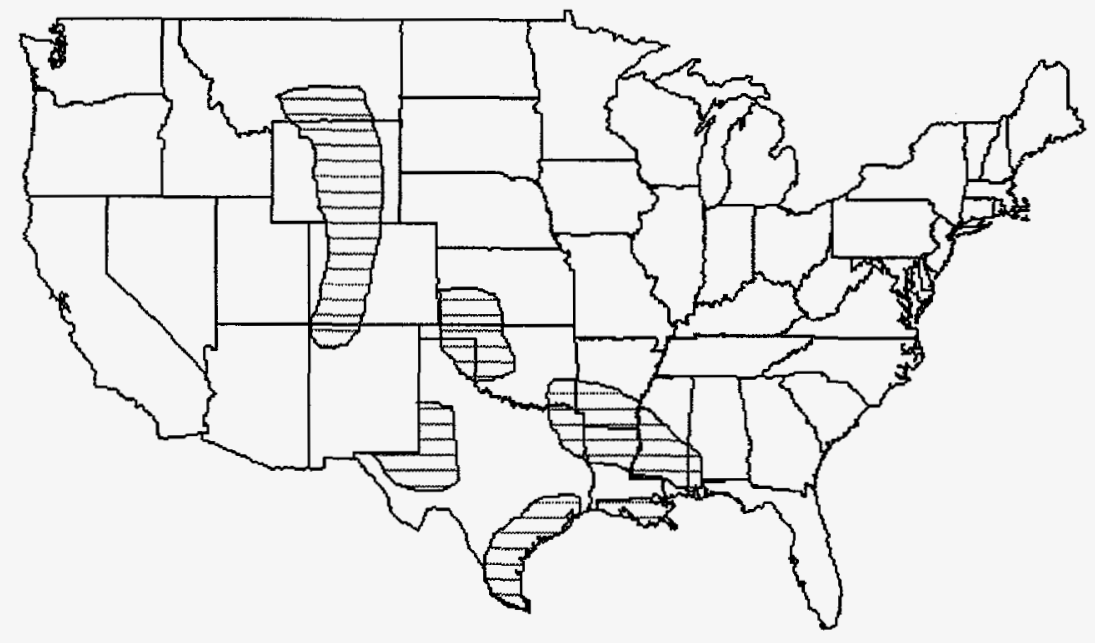

Source: Energy Information Administration, Office of Oil and Gas. 


\section{Indexing Procedure}

The leading supply, service, and contracting companies (active in one or more of the regions) were contacted every year (1976 through 1997) for local June prices for their component of equipment or operating function. The objective of this process was to acquire prices that were representative for each region. Total component costs were determined from these prices and summed to permit indexing.

The indices in this report reflect changes in the costs of items, and their related transportation charges and associated installation costs on representative leases. The index numbers in this report are "pure" cost indices. A pure cost index measures the change in cost of a fixed quantity of goods and/or services. Pure cost indices are applied to the individual line items presented in the appendix tables.

The subtotal and total indices are composite indices. A composite index measures the change in cost of an aggregate of goods and/or services. Any equipment changes that may be made due to technological advances will be reflected in the composite indices.
The annual operating cost indices measure the change in direct costs incident to the production of oil and gas and exclude changes in indirect costs, such as depreciation and ad valorem and severance taxes.

The indices are calculated with 1976 as the base year as follows: 1997 index $=(1997$ costs $/ 1976$ costs $) \times 100$.

Annual percentage changes can be determined by dividing the last year's index by the prior year's index, subtracting one (1.0), and multiplying by 100 . For example, to find the percent change from 1996 to 1997 , divide the 1997 index by the 1996 index, subtract one (1.0), and multiply by 100 .

The estimated region equipment costs for the representative oil leases were summed, averaged, and indexed by depth, providing a general measure of equipment expenditures relative to depth. The estimated region equipment costs for the representative gas leases were also summed, averaged, and indexed by depth and by production rate. The aggregate average cost for all regions and depths were indexed to allow general trend analyses by year. This same procedure was applied to the annual operating costs for the formulated oil leases and for the formulated gas leases. 



\section{Discussion of Results}

The summary of composite data and the detailed appendix tables permit analysis of equipment and operating costs for each region, depth, method, and type of production. The data in this report should be considered as revised, except for the 1997 data, which are preliminary. Some of the revisions which appear in this report affected equipment costs for the entire series, beginning in 1976. Though these were small, in most cases, the equipment cost revisions reflected a minor change in operating costs. There were no major revisions. The following is a discussion of the composite costs and indices.

\section{Overview}

This report continues a data series begun in 1976, providing a history of equipment and operating costs for oil and gas leases from 1976 through 1997. (See Appendix $N$ for both nominal and deflated full-series data). Figure 3 shows indices of the aggregate average costs for oil well equipment and operations, indicating general upward pressure on costs. The period of rapid cost increase which began before 1976 changed in 1982, the peak year for total equipment costs, which was followed by prices rising and falling within a range somewhat near the 1982 level. Operating costs followed a different path. The post-1982 drop was minimal, and the 1982 level has been exceeded each year since 1989. Operating costs for 1997 are at the all-time peak. These have been largely influenced by energy costs (natural gas and electric power) and the costs of oil field services such as well servicing units and chemicals. Labor has also been a factor in many areas.

Figure 4 is a plot of tubing costs for 10-well oil leases. The type of tubing used for deeper wells not only costs more than that used for shallower wells, but their price variations have been more extreme. Prices for 12,000-foot wells, which dropped from 1992 to 1994 , have risen since. Costs for shallower wells have generally followed the same trend.

Figure 5 is a plot of oil lease equipment costs excluding tubing. Contrasting Figures 4 and 5 show that the non-tubing equipment costs vary much less than those for tubing. However, the 1982 non-tubing equipment costs were nearly double the 1976 costs. Non-tubing equipment costs declined from 1982 through 1987 and have generally increased since.

The 1997 index of non-tubing equipment cost for all depths is about 212 , which is 112 percent higher than the 1976 cost. Since 1976, the non-tubing cost for 8,000-foot wells was exceeded by that for 12,000-foot wells about one-third of the

Figure 3. Aggregate Average Cost Indices for Primary Oil Recovery, 1976-1997

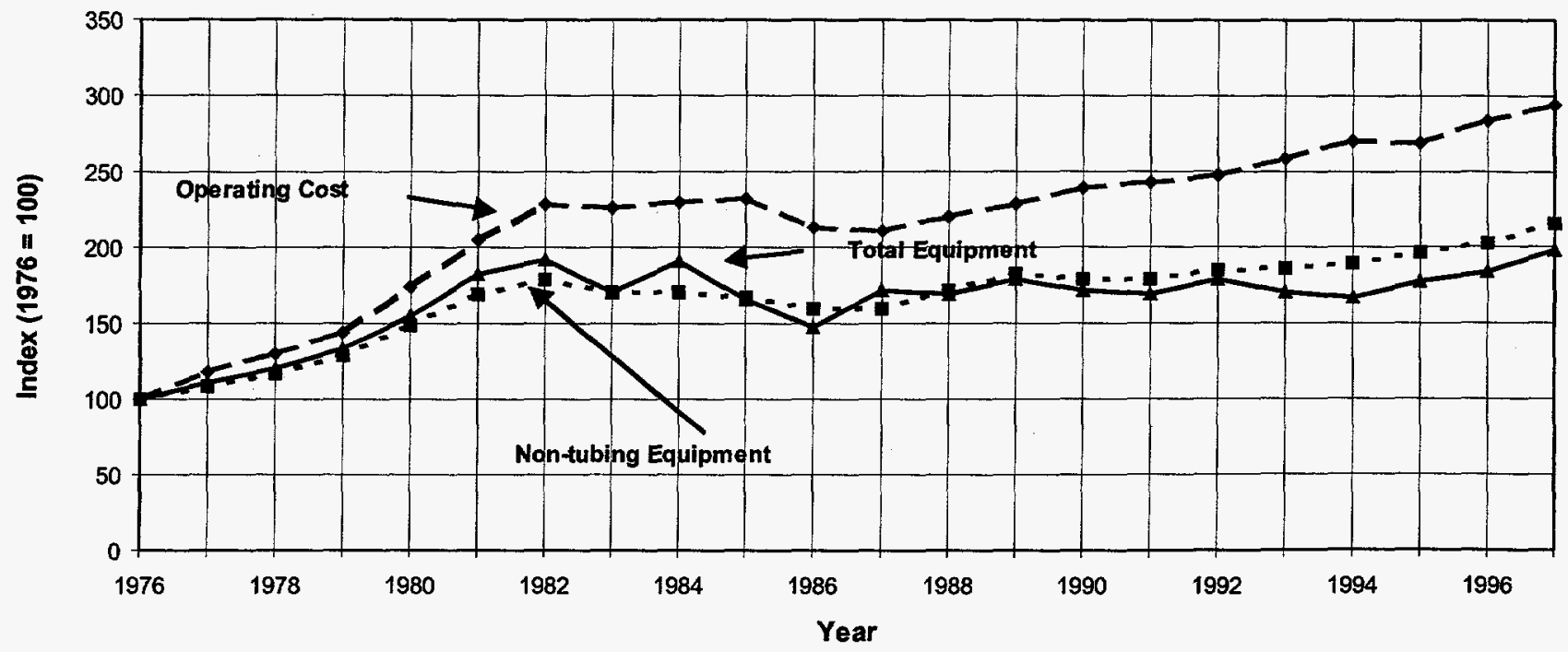

Source: Energy Information Administration, Office of Oil and Gas 
time, an anomaly related to the mixture of pumping equipment types used for 8,000-foot wells.

The availability of well service units (WSUs) is widely used as an indicator of price pressures on operating costs. When WSU utilization is high, prices of other operating cost items are usually firm. The active WSU count rose from about 2,600 in 1976 to 4,850 in 1981, when activity levels peaked (see Figure 6). Although the 1992 active WSU count dropped to near 1976 levels, later counts show a modest increase. At the same time, available WSUs dropped to 1980 levels. Pressure on the well service industry was the result of overbuilding in the early 1980 's. With a peak of about 8,000 WSU's available in 1985, the portion of WSUs at work was less than 60 percent. In 1986, working WSUs were only 40 percent of those available, and 1997 surveys reflect that 71 percent of the WSUs were working. Industry reports show that there are labor constraints that limit the current level of active WSUs, thus indicating industry-wide price increases to cover not only labor, but repair and replacement of equipment.

Figure 7 contains the equipping and operating cost indices for gas wells (note that gas well equipment costs do not include tubing costs). The index for gas equipment costs increased steadily from 1976 to a peak of about 183 in 1982. Lower levels of activity forced the index to a low of 153 in 1986, from which time costs increased to set new highs between 1990 and 1993, with a slight drop to 1994. The trend from 1994 to 1997 has been upward. Operating costs have set new highs beginning with 1990 , and rose at a steadier pace than equipment costs because of recent changes in labor prices, which are a major influence on the overall costs of gas well operation. The use of gas for fuel on gas leases is relatively insignificant, so increasing gas prices had little effect.

\section{Equipment Costs for Oil Leases}

\section{Primary Recovery}

Table 1 is a summary of the composite lease equipment costs and indices for primary oil recovery operations in 6 onshore producing regions by depth. The trends in costs varied by depth and region. The aggregate (or sum) of the 10-well oil lease equipment costs for the six regions and 4 depths increased 7.5 percent in the period from 1996 to 1997 . Table 1 also presents the average costs and indices of the 6 regions by depth. As shown in Figure 8, the average equipment costs increase with depth.

The annual increases are greater for deeper wells, as might be expected. Although there are regional differences in equipment costs for each depth of wells, the range of indexed values is larger for operating costs. The significant fact is that costs for primary oil equipment and operations rose 7.5 percent and 3.9 percent, respectively.

\section{Secondary Recovery}

Table 2 summarizes the additional lease equipment costs and indices associated with secondary oil recovery (waterflood) from depths of 2,000, 4,000, and 8,000 feet in west Texas. This region was the focus of a substantial part of the early secondary recovery work in the country, and the differences between primary and secondary costs are presumed to be similar to those in other regions. The method used in this report is waterflooding. The additional lease equipment is the equipment needed to convert from a primary recovery operation to a secondary recovery operation. The aggregate increase in additional equipment costs was about 18 percent for the 1996-1997 period. As noted before, drilling cost estimates are subject to major annual revisions and, as drilling costs can account for more than one-half of the additional equipment costs, revisions to drilling costs may obscure the changes in other costs. The 1997 drilling costs reflect the end of over-capacity in the industry, and limitations of equipment, personnel, and auxiliary services add to constrain activity in some areas. Figure 9 shows the additional costs of waterflood equipment for depths of 2,000, 4,000, and 8,000 feet for 1994 through 1997.

\section{Operating Costs for Oil Leases}

\section{Primary Recovery}

Table 3 is a summary of the annual operating costs and indices for primary oil recovery operations, which are shown in Figure 10, while those for secondary operations are shown in Figure 11. The average for the aggregate of the operating costs for the 6 regions and 4 depths was $\$ 228,600$ for the 10-well lease in 1997. This represents about a 3.9 percent increase over the average for 1996. Examination of Table 3 shows that most costs for oil operations rose from 1996 to 1997. The 1976 to 1997 history of aggregate operating costs is shown in Figure 3. The upward trend in operating costs began in 1988 .

Changes in individual components of operating costs show large variations. Fuel, power and water costs comprise one of the most volatile components for oil leases, primarily due to changes in the average price of natural gas in the different regions. Overall costs for fuel, power and water ranged from increases of from 4 to 6 percent for the 1996-1997 period for all regions except California and the Rocky Mountains. The increase for the Rocky Mountain region was about 1 to 3 percent, while California costs dropped by 2 to 4 percent. Contrasted with the rest of the nation, the primary energy source for California and the Rocky Mountains regions is electricity. In the Rocky Mountains, electricity is generated by coal-fired plants and hydroelectric plants that have adequate water supplies. In California, hydroelectric plants 
Figure 4. Tubing Costs for Oil Leases, 1976-1997 (10 Producing Wells)

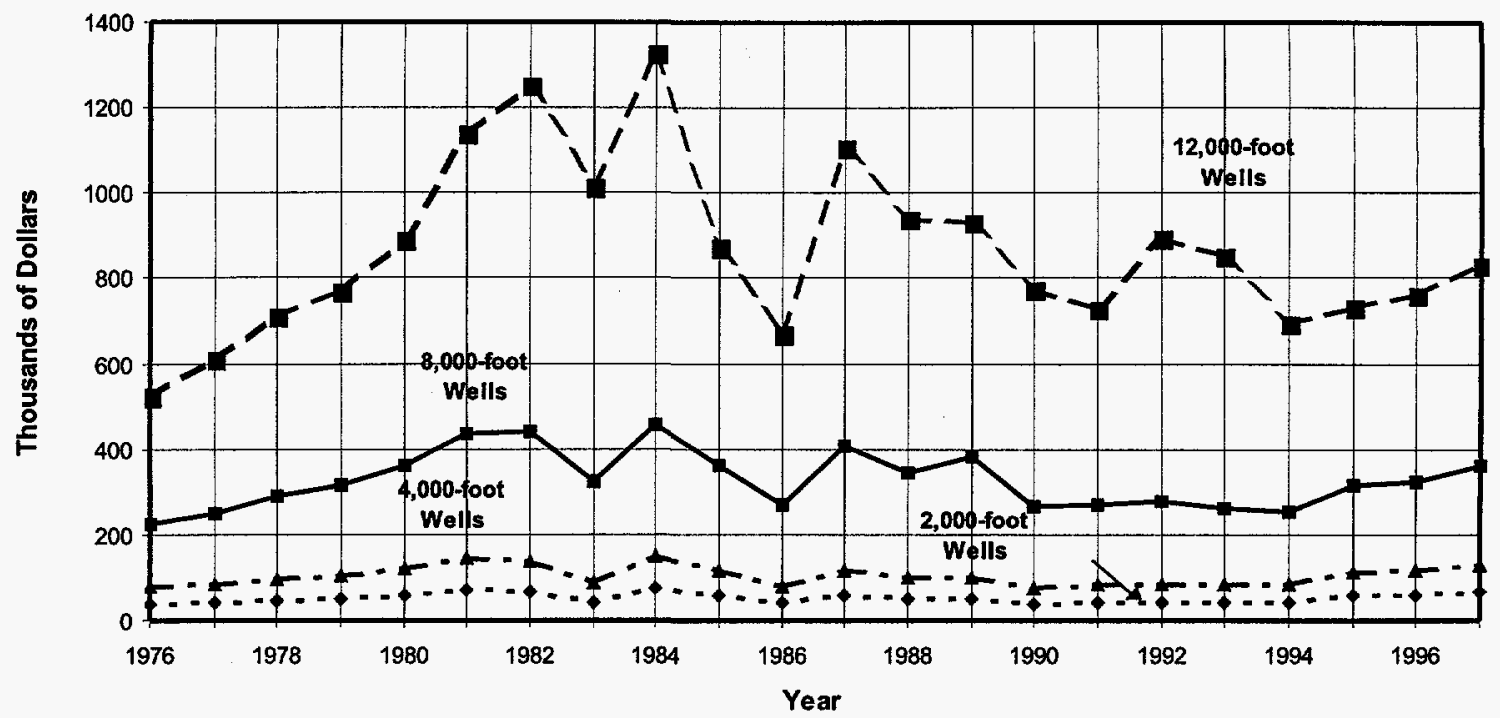

Source: Energy Information Administration, Office of Oil and Gas.

Figure 5. Non-tubing Equipment Costs for Oil Leases, 1976-1997 (10 Producing Wells)

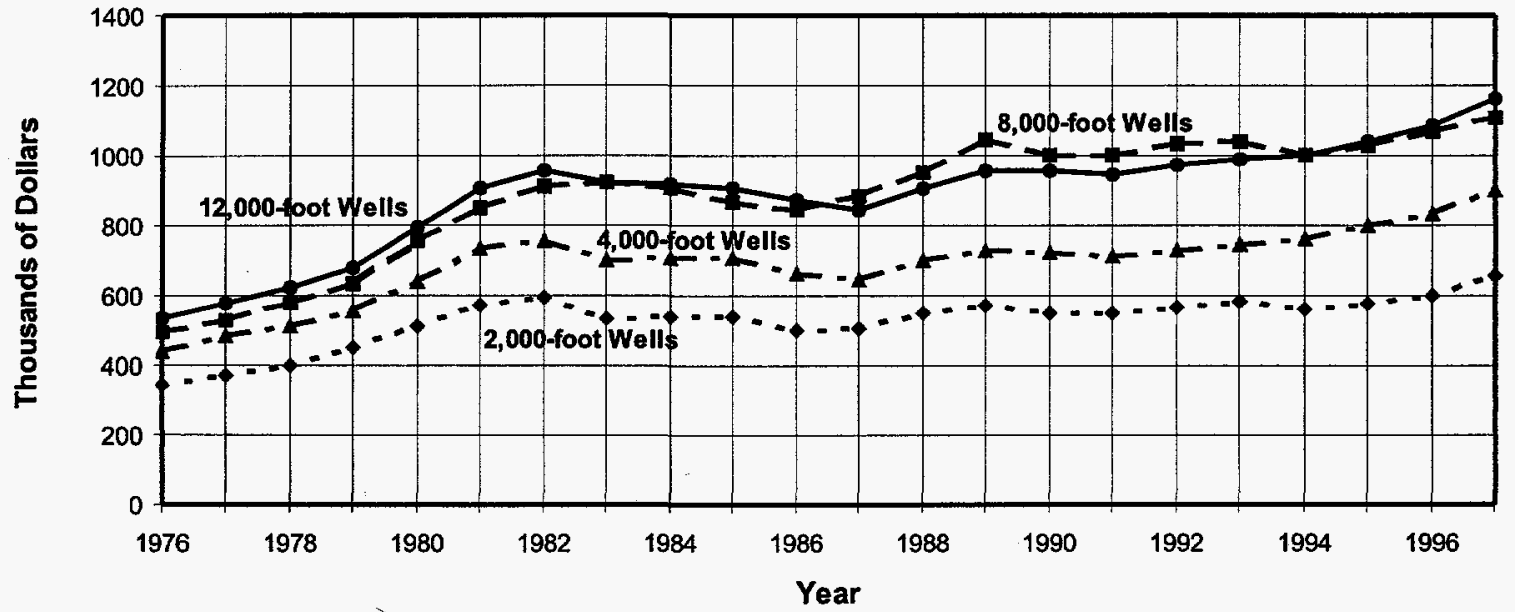

Source: Energy Information Administration, Office of Oil and Gas. 
Figure 6. Well Service Units, 1976-1997

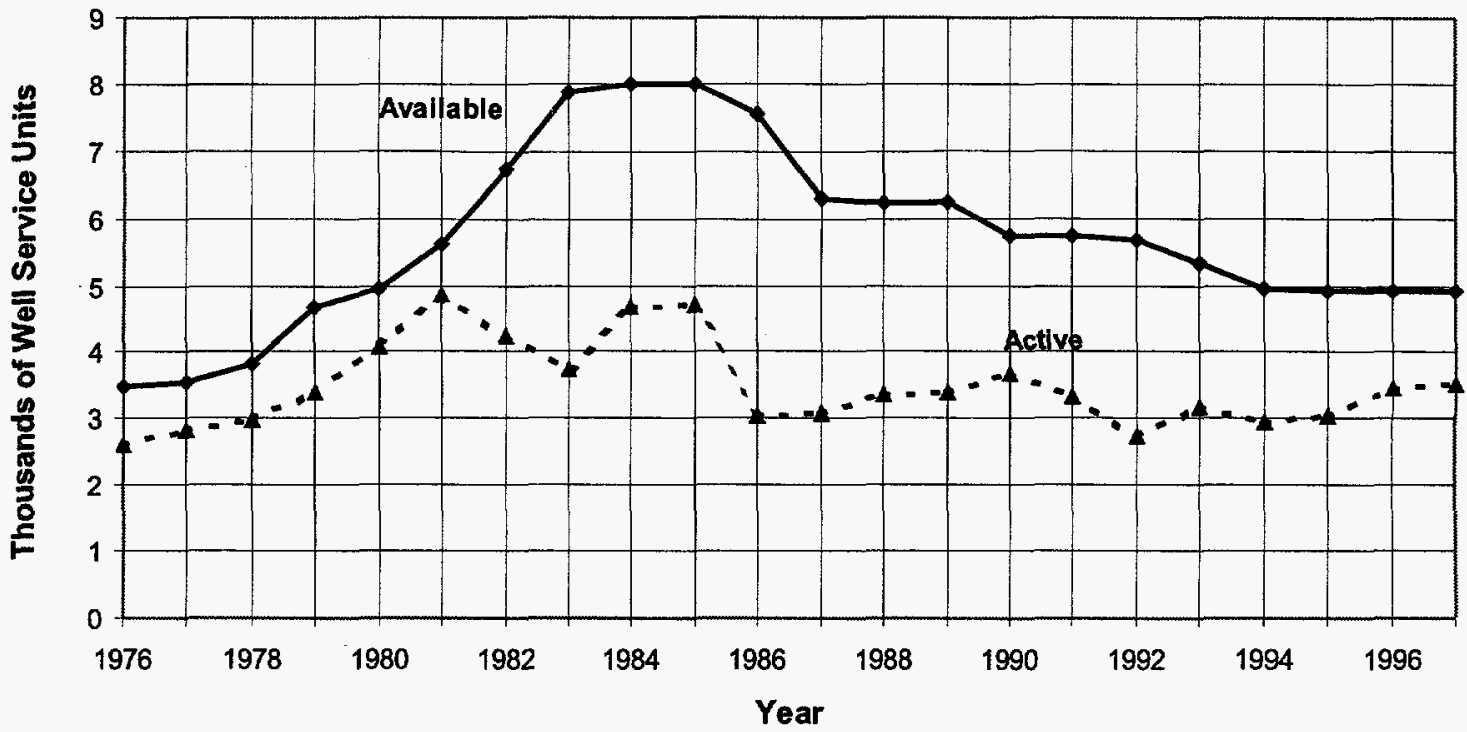

Source: Energy Information Administration, Office of Oil and Gas.

Figure 7. Aggregate Average Cost Indices for Gas Recovery, 1976-1997

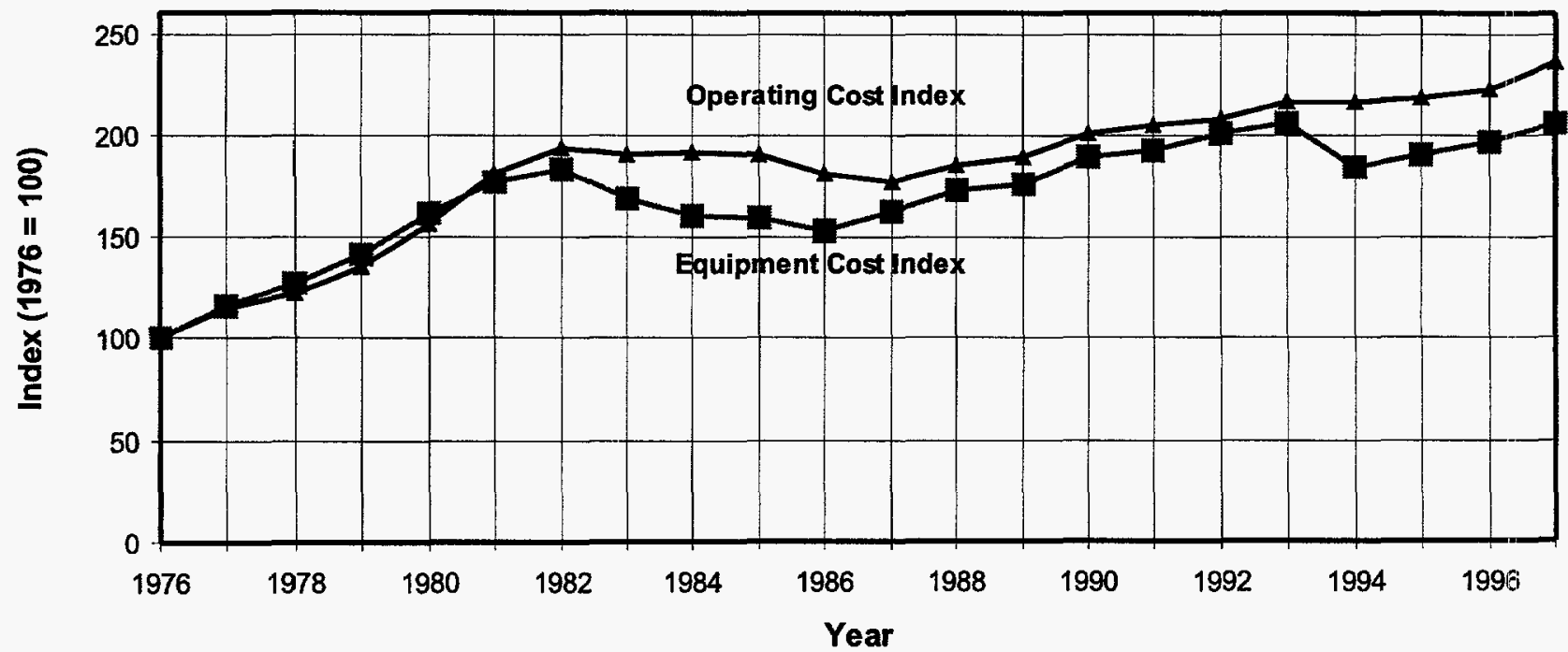

Source: Energy Information Administration, Office of Oil and Gas. 
Table 1. Summary of Lease Equipment Costs and Composite Indices for Primary Oil Recovery Operations (10 Producing Wells)

\begin{tabular}{|c|c|c|c|c|c|}
\hline \multirow[b]{2}{*}{ Area } & \multicolumn{4}{|c|}{ Index $(1976=100)$} & \multirow{2}{*}{$\begin{array}{c}1997^{\star} \\
\text { Cost } \\
\text { (dollars) }\end{array}$} \\
\hline & 1994 & 1995 & 1996 & 1997 & \\
\hline & \multicolumn{5}{|c|}{ 2,000-Foot Wells } \\
\hline California...................................... & 166.7 & 177.7 & 185.2 & 200.0 & $1,003,600$ \\
\hline 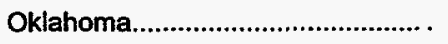 & 174.1 & 185.1 & 191.9 & 210.4 & 736,100 \\
\hline South Louisiana............................... & 181.1 & 192.2 & 201.1 & 220.3 & 815,600 \\
\hline 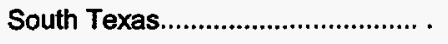 & 179.3 & 190.1 & 196.6 & 213.8 & 760,000 \\
\hline West Texas........................................ & 175.5 & 186.3 & 192.9 & 210.2 & 741,800 \\
\hline Rocky Mountains............................ & 171.8 & 181.8 & 189.3 & 211.5 & 745,500 \\
\hline \multirow[t]{2}{*}{ Average or Index....................... } & 174.3 & 185.1 & 192.4 & 210.4 & 800,400 \\
\hline & \multicolumn{4}{|c|}{ 4,000-Foot Wells } & \\
\hline 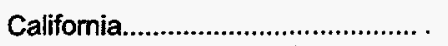 & 146.7 & 158.0 & 165.0 & 177.7 & $1,247,900$ \\
\hline Oklahoma....................................... & 161.4 & 178.0 & 185.3 & 192.5 & 969,200 \\
\hline South Louisiana............................ & 192.0 & 204.2 & 212.7 & 241.2 & $1,027,300$ \\
\hline South Texas.................................. & 182.0 & 192.7 & 199.3 & 224.2 & 976,500 \\
\hline West Texas................................. & 160.1 & 176.4 & 183.5 & 189.6 & 963,900 \\
\hline Rocky Mountains........................... & 154.5 & 170.5 & 177.6 & 193.5 & 982,200 \\
\hline \multirow[t]{2}{*}{ Average or Index......................... } & 163.8 & 177.7 & 184.9 & 200.0 & $1,027,800$ \\
\hline & \multicolumn{4}{|c|}{ 8,000-Foot Wells } & \\
\hline 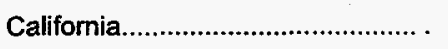 & 165.9 & 171.7 & 176.0 & 184.4 & $1,683,200$ \\
\hline Oklahoma............................................ & 175.1 & 190.4 & 197.6 & 210.3 & $1,541,000$ \\
\hline South Louisiana.............................. & 188.0 & 204.2 & 213.0 & 241.8 & $1,332,500$ \\
\hline South Texas................................... . & 175.0 & 189.2 & 195.9 & 220.7 & $1,246,300$ \\
\hline 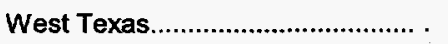 & 156.8 & 173.6 & 181.3 & 189.7 & $1,683,100$ \\
\hline Rocky Mountains........................... & 144.8 & 158.9 & 165.9 & 182.5 & $1,639,100$ \\
\hline \multirow[t]{2}{*}{ Average or Index......................... . } & 165.3 & 178.7 & 185.5 & 200.7 & $1,520,900$ \\
\hline & \multicolumn{4}{|c|}{ 12,000-Foot Wells } & \\
\hline California..................................... & 163.3 & 169.6 & 175.0 & 182.8 & $2,058,400$ \\
\hline Oklahoma......................................... & 163.8 & 170.3 & 176.5 & 187.9 & $1,824,800$ \\
\hline South Louisiana............................ & 169.1 & 176.3 & 184.4 & 197.6 & $2,040,100$ \\
\hline South Texas................................. & 169.3 & 175.5 & 182.6 & 192.5 & $1,954,300$ \\
\hline 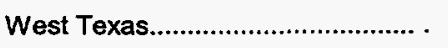 & 164.7 & 171.1 & 177.1 & 187.2 & $1,860,600$ \\
\hline Rocky Mountains............................. & 166.6 & 173.3 & 179.7 & 189.1 & $1,859,700$ \\
\hline Average or Index.......................... & 166.1 & 172.6 & 179.2 & 189.5 & $1,933,000$ \\
\hline Aggregate Average......................... & 166.6 & 177.1 & 183.9 & 197.7 & $1,320,500$ \\
\hline
\end{tabular}

- Preliminary

Note: Reported average or aggregate average indices are indices of the average costs. They are not an average of the index values.

Source: Energy Information Administration, Office of Oil and Gas 
Figure 8. Aggregate Average Lease Equipment Costs for Primary Oil Recovery,

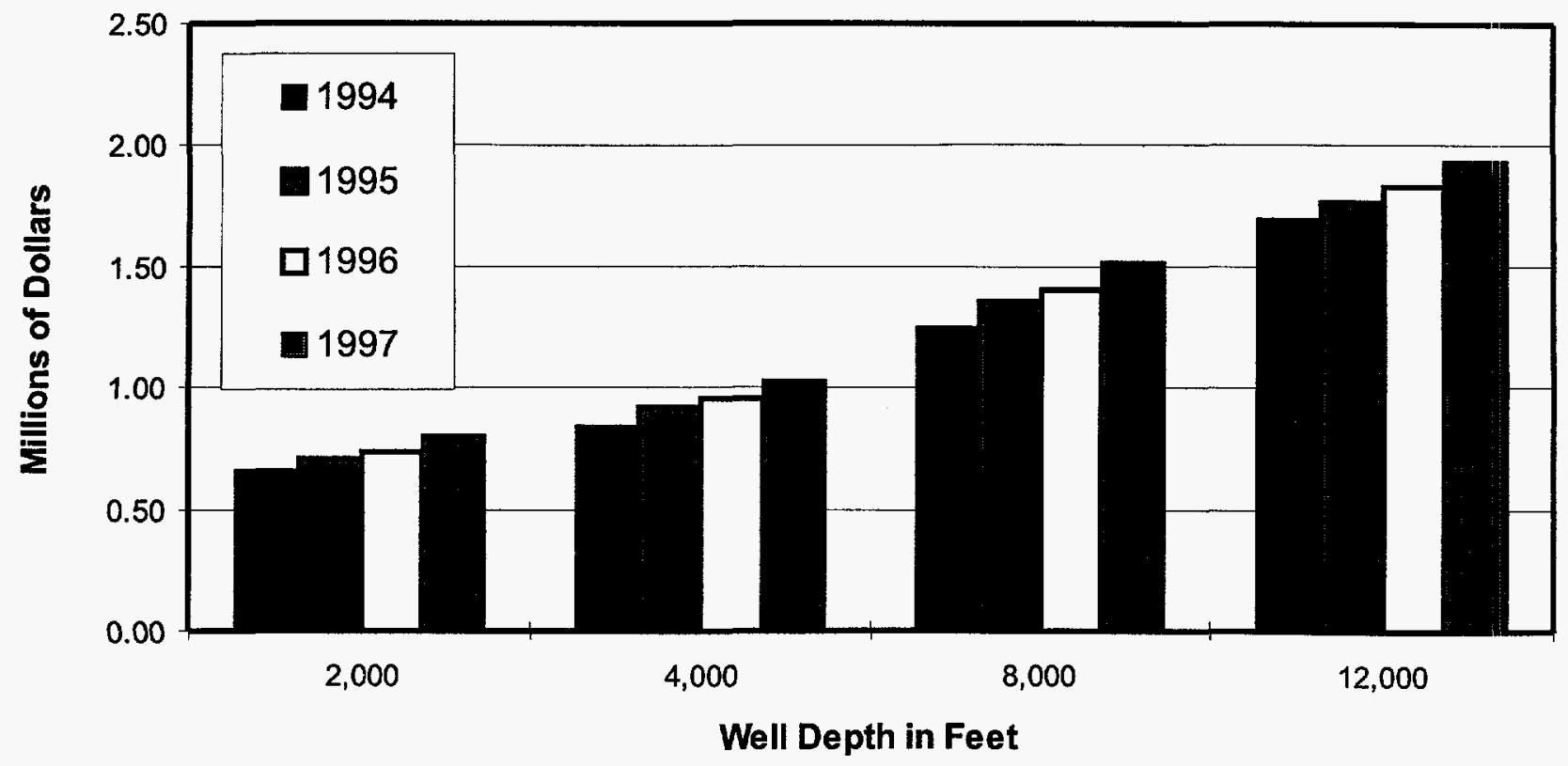

Source: Energy Information Administration, Office of Oil and Gas.

Figure 9. Additional Cost of Lease Equipment for Secondary Recovery in West Texas

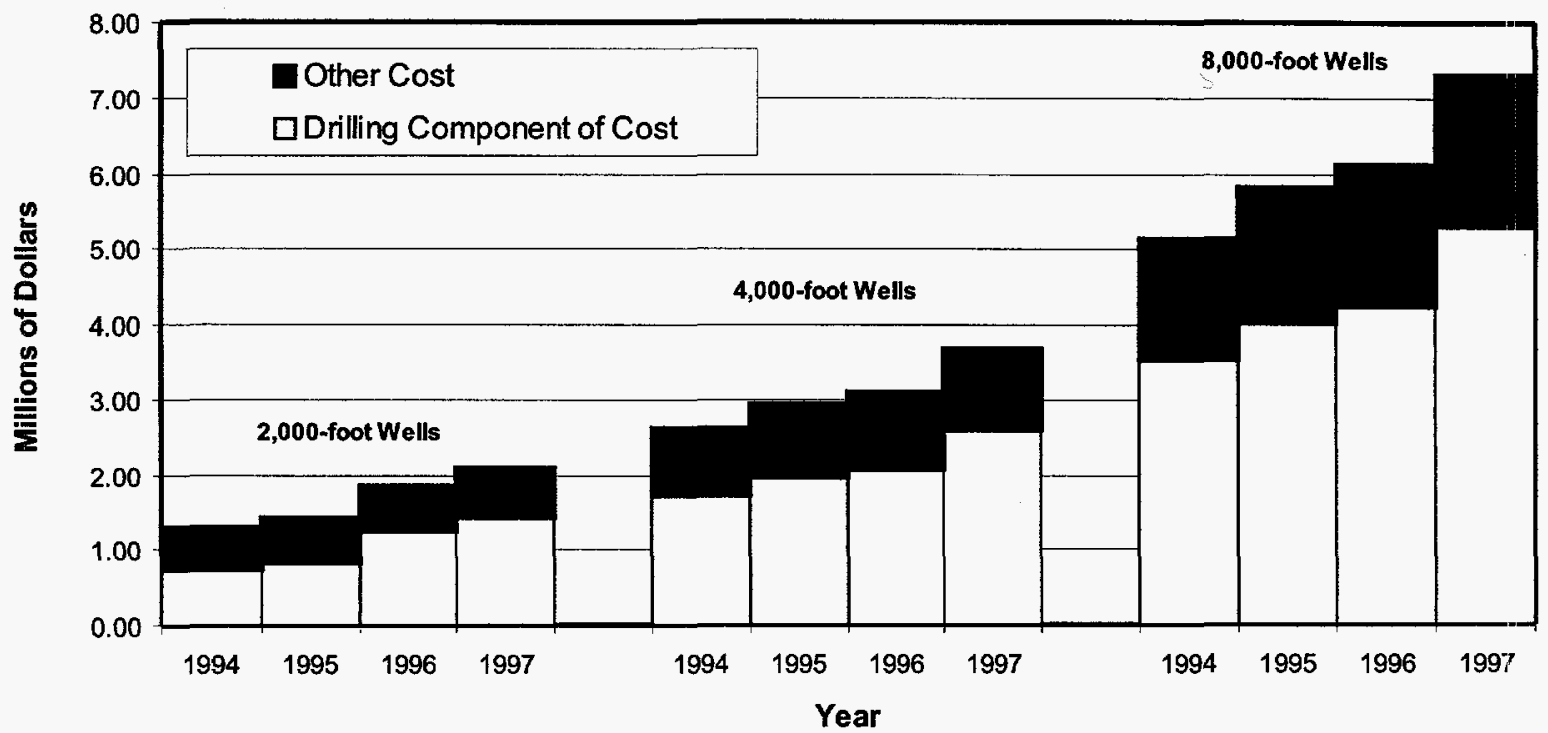

Source: Energy Information Administration, Office of Oil and Gas. 
Table 2. Summary of Additional Costs and Composite Indices for Lease Equipment and Injection Wells in West Texas for Secondary Oil Recovery

\begin{tabular}{|c|c|c|c|c|c|}
\hline \multirow[b]{2}{*}{ Component } & \multicolumn{4}{|c|}{ Index $(1976=100)$} & \multirow{2}{*}{$\begin{array}{c}1997^{*} \\
\text { Cost } \\
\text { (dollars) }\end{array}$} \\
\hline & 1994 & 1995 & 1996 & 1997 & \\
\hline & \multicolumn{5}{|c|}{ 2,000-Foot Wells } \\
\hline 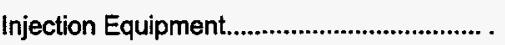 & 225.4 & 238.3 & 244.3 & 255.4 & 501,000 \\
\hline 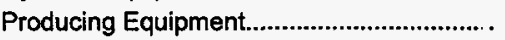 & 139.7 & 157.8 & 159.7 & 191.3 & 154,600 \\
\hline Injection Wells ${ }^{* \star}$ & 138.3 & 157.0 & 235.5 & 270.8 & $1,434,900$ \\
\hline \multirow[t]{2}{*}{ 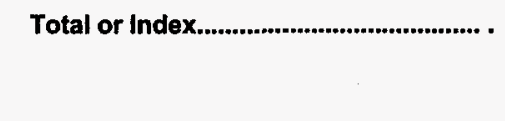 } & 159.7 & 176.9 & 230.1 & 259.2 & $2,090,500$ \\
\hline & \multicolumn{5}{|c|}{ 4,000-Foot Wells } \\
\hline 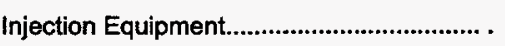 & 214.0 & 226.2 & 232.0 & 242.5 & 501,000 \\
\hline 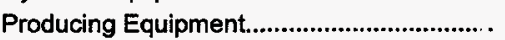 & 131.1 & 153.8 & 162.6 & 180.6 & 595,600 \\
\hline 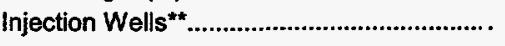 & 152.8 & 173.7 & 182.4 & 228.0 & $2,588,800$ \\
\hline \multirow[t]{2}{*}{ 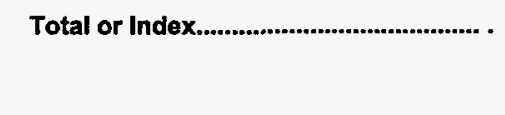 } & 156.1 & 176.3 & 184.6 & 220.5 & $3,685,400$ \\
\hline & \multicolumn{5}{|c|}{8,000 -Foot Wells } \\
\hline 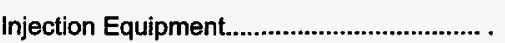 & 218.0 & 232.1 & 237.9 & 249.6 & 847,000 \\
\hline Producing Equipment....................................... & 128.5 & 148.3 & 156.9 & 172.3 & $1,184,700$ \\
\hline 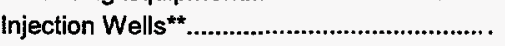 & 118.3 & 135.1 & 141.9 & 177.3 & $5,285,300$ \\
\hline Total or Index & 128.5 & 145.6 & 152.6 & 182.6 & $7,317,000$ \\
\hline Aggregate Average.................................... & 139.5 & 157.4 & 170.5 & 201.9 & $4,364,300$ \\
\hline
\end{tabular}

* Preliminary

* Costs from Joint Association Survey data.

Note: Reported average or aggregate average indices are indices of the average costs. They are not an average of the index values.

Source: Energy Information Administration, Office of Oil and Gas

operate at full generating capacity as long as there is an ample water supply. However, their water supply is weather-dependent with little or no reserve,so a dry winter can cause reduction in hydroelectric generation of electricity the following year. The reduction in hydroelectric capacity is made up by power from generators using alternate fuels, or is purchased from out-of-State sources. Therefore, electric rates can vary widely in California. Figure 12 depicts fuel, power, and water indices for 12,000-foot wells in the Rocky Mountains and California. Note that the fuel, power, and water costs decreased from 1994 to 1997 for California, while the Rocky Mountain region costs rose. Even with the recent cost increases in the Rocky Mountains, the period since 1984 shows costs have remained in a narrow range, unlike costs in California, which have trended upward since 1976.

\section{Secondary Recovery}

Table 4 provides a summary of the composite secondary oil recovery operating costs for west Texas. The average aggregated lease costs ( 10 producing and 11 injection wells) for all depths rose less than 2 percent from 1996 to 1997. Fuel, power, and water costs for secondary recovery operations increased by about 4 percent for the 1994-1997 period and costs for primary recovery operations in this region increased about 49 percent for the same period. The differences in the changes for fuel, power, and water costs occurred because engines powered by natural gas were the prime movers for primary recovery operations in this region, and electric motors were used for secondary recovery operations. As an example, Figure 13 shows fuel, power, and 
water cost indices for primary and secondary oil recovery in west Texas for 4,000-foot wells. Only 6 years of the 1976 to 1997 period indicated indices in excess of those for primary oil operation.

\section{Offshore Operations}

Table 5 presents a summary of annual operating costs and composite indices for offshore production operations in the Gulf of Mexico for wells with a true vertical depth of 10,500 feet. Production from offshore installations includes large gas volumes compared to the volumes in the average onshore lease, a fact that is reflected in operating costs. The aggregate average of costs at all water depths decreased by about 2 percent from 1996 to $\$ 3,693,500$ per platform in 1997 .

\section{Equipment Costs for Gas Leases}

Equipment and operating costs for gas leases producing from depths of $2,000,4,000,8,000,12,000$, and 16,000 feet, were determined for 6 onshore regions of the lower 48 States (Figure 2). For each region and depth, costs and indices for equipment for a one-well lease were determined for representative or average gas production rates. Costs and indices were also calculated for a higher and, where possible, for a lower production rate. Composite indices and costs for equipment are presented for a one-well lease with production rates of 50,250,500,1,000, 5,000, and 10,000 Mcf of gas per day by depth and region. Figure 14 displays the average equipment costs by rate of production and well depth for 1997. There is a large difference between the equipment costs for some production rates and depths, such as wells of various depths producing $250 \mathrm{Mcf}$ per day. This difference is the result of variations in the type and size of equipment needed in different regions, for different depths, and for different production rates. For example, dehydrators and line heaters are needed in cold climates but may not be needed in more temperate climates.

The indices for the aggregate costs of gas lease equipment for all depths and regions rose 4.8 percent for the 1996-1997 period. Table 6 shows that the overall aggregate average gas lease had an equipment cost of $\$ 47,000$ in 1997.

Tables 7 through 12 present summaries of composite gas lease equipment costs and indices for a given production rate by depth and region. For each production rate, the costs are summed and averaged for the selected regions and depths. These average costs and the corresponding indices are presented in each table. The 1996-1997 change in equipment costs ranged from an increase of 4 percent for wells producing $50 \mathrm{Mcf}$ of gas per day to an increase of about 6 percent for wells flowing $5 \mathrm{MMcf}$ of gas per day.
Table 13 contains gas lease equipment costs aggregated by depth. Changes in gas equipment costs from 1996 to 1997 were positive for all wells, and ranged from increases of 4.1 to 5.4 percent. The dominant factor in determining gas well equipment costs is the production capacity of the equipment. Figure 15 illustrates the aggregate average gas well equipment costs for 1994 through 1997 by production rate. The stair-step appearance of the costs for each production rate shows greater year-to-year variation for higher flow rates.

\section{Operating Costs for Gas Leases}

Operating costs for gas leases aggregated for all depths, regions, and production rates are shown in Table 14. There was an increase of 5.9 percent from 1996 to 1997 , to $\$ 25,000$. Tables 15 through 20 are summaries of composite costs and indices for operating a gas lease. Each table is a summary for: one production rate for the same depth and region used for: lease equipment costs. For each depth and production rate, the individual operating costs by region were averaged and indices were calculated. From 1996 to 1997, wells producing; 5,000 and 10,000 Mcf per day exhibited an operating cosi: increase of about 4.6 percent, while costs for wells producing. at a rate of $250 \mathrm{Mcf}$ per day rose at about 6.5 percent.

Well depth has more effect on gas well operating costs than. on equipment costs, since depth is a major factor in the cost: of down-hole repairs, the amount of chemicals used, and other maintenance cost components. However, the changes in operating costs aggregated by depth from 1996 through 1997 show little variation across time. The annual changes ranged from 5.7 to 6.8 percent from 1996 to 1997 . The annual gas well operating costs aggregated by depth are shown in Table 21 .

Figure 17 depicts the aggregate average annual gas well operating costs by depth and producing rate for 1997. Operating costs decreased as the producing rate increased from 250 to 500 thousand cubic feet of gas per day in 8,000 -and 12,000-foot wells. This is a result of the well design and the completion techniques used. Wells producing at 500 thousand cubic feet of gas per day, or more, were considered to be completed with packers. Packers protect the casing-tubing annulus and the casing wellhead from the bottom-hole pressure and any corrosive properties of the well's fluids. With these flow rates, the tubing flow velocity is sufficient to remove the well liquids which accumulate in the tubing. Either tubing displacement or corrosion inhibitor squeeze jobs can be used to protect the production string from corrosion or scale deposition. Wells producing at rates of 250 thousand cubic feet of gas per day or less have lower tubing flow velocities, which are not always adequate to remove accumulated liquids from the well. Increasing liquid levels usually causes reduced gas production. Therefore, these wells were considered to be completed without packers 
to permit fluids to be forced up the tubing by expansion of the compressed gas in the casing-tubing annulus. Because the gas wells which produce at lower flow rates have no packers, the casing-tubing annulus is exposed to the corrosive properties of the well fluids and often needs chemical protection. Tubing displacement and corrosion inhibitor squeeze jobs are not effective without a packer, or making them effective would be cost prohibitive. Therefore, continuous chemical injection down the casing-tubing annulus is a common practice. This involves surface chemical injection pumps, maintenance, and larger volumes of chemicals. As a result, wells which produce less than 250 thousand cubic feet of gas per day have higher fuel, chemical and disposal costs, and higher surface maintenance 
Table 3. Summary of Direct Annual Operating Costs and Composite Indices for Primary Oil Recovery Operations (10 Producing Wells)

\begin{tabular}{|c|c|c|c|c|c|}
\hline \multirow[b]{2}{*}{ Area } & \multicolumn{4}{|c|}{ Index $(1976=100)$} & \multirow{2}{*}{ 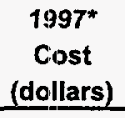 } \\
\hline & 1994 & 1995 & 1996 & 1997 & \\
\hline & \multicolumn{5}{|c|}{ 2,000-Foot Wells } \\
\hline 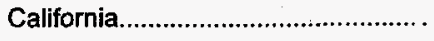 & 270.6 & 269.8 & 271.5 & 275.7 & 150,800 \\
\hline Oklahoma............................................... & 247.9 & 247.2 & 266.5 & 285.4 & 127,000 \\
\hline South Louisiana............................... . & 250.4 & 248.3 & 263.2 & 286.0 & 155,600 \\
\hline 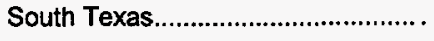 & 245.4 & 245.4 & 256.1 & 262.1 & 143,900 \\
\hline West Texas........................................ & 236.2 & 235.5 & 248.9 & 256.1 & 116,800 \\
\hline Rocky Mountains.............................. . & 219.4 & 220.8 & 232.7 & 247.4 & 137,800 \\
\hline \multirow[t]{2}{*}{ Average or Index............................ } & 245.2 & 244.8 & 256.4 & 268.8 & 138,700 \\
\hline & \multicolumn{5}{|c|}{ 4,000-Foot Wells } \\
\hline 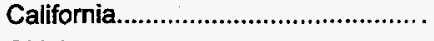 & 291.9 & 287.5 & 288.0 & 289.6 & 199,800 \\
\hline Oklahoma............................................. & 246.9 & 246.9 & 269.3 & 288.1 & 147,500 \\
\hline South Louisiana................................ & 246.7 & 243.0 & 261.8 & 290.9 & 227,500 \\
\hline 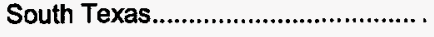 & 243.2 & 232.5 & 243.7 & 254.5 & 200,300 \\
\hline 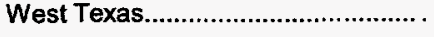 & 233.6 & 233.8 & 248.9 & 256.8 & 136,100 \\
\hline Rocky Mountains............................. . & 213.7 & 216.8 & 228.2 & 242.4 & 155,400 \\
\hline \multirow[t]{2}{*}{ Average or Index............................ } & 246.9 & 243.7 & 256.6 & 270.6 & 177,800 \\
\hline & \multicolumn{5}{|c|}{ 8,000-Foot Wells } \\
\hline 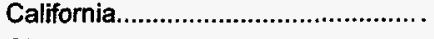 & 380.1 & 371.4 & 371.7 & 367.6 & 340,400 \\
\hline 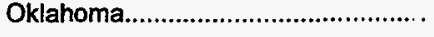 & 293.0 & 295.2 & 322.6 & 336.6 & 254,100 \\
\hline South Louisiana................................. . & 253.0 & 249.7 & 268.3 & 298.8 & 271,600 \\
\hline 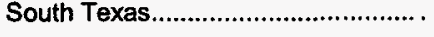 & 240.8 & 238.6 & 252.6 & 264.4 & 245,400 \\
\hline West Texas............................................ & 232.3 & 231.4 & 246.1 & 254.8 & 188,300 \\
\hline Rocky Mountains................................ . & 207.6 & 209.8 & 213.6 & 224.3 & 198,300 \\
\hline \multirow[t]{2}{*}{ Average or Index............................. } & 268.7 & 266.7 & 279.5 & 291.4 & 249,700 \\
\hline & \multicolumn{5}{|c|}{ 12,000-Foot Wells } \\
\hline 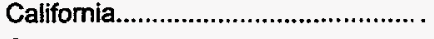 & 395.4 & 384.2 & 385.6 & 379.6 & 494,600 \\
\hline 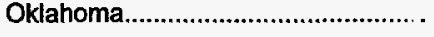 & 294.9 & 295.2 & 325.3 & 339.8 & 304,100 \\
\hline South Louisiana.................................. & 274.2 & 270.6 & 292.1 & 308.7 & $35,4,400$ \\
\hline 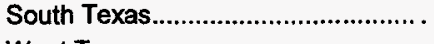 & 271.1 & 272.3 & 290.7 & 298.0 & 355,800 \\
\hline 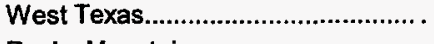 & 269.5 & 269.8 & 292.3 & 300.0 & 283,200 \\
\hline Rocky Mountains.............................. & 267.0 & 272.4 & 286.4 & 296.0 & 297,800 \\
\hline Average or Index............................ & 298.9 & 297.2 & 314.2 & 321.9 & 348,300 \\
\hline Aggregate Average.......................... . & 270.7 & 268.8 & 282.9 & 293.8 & 228,600 \\
\hline
\end{tabular}

- Preliminary

Note: Reported average or aggregate average indices are indices of the average costs. They are not an average of the index values.

Source: Energy Information Administration, Office of Oil and Gas 
Figure 10. Aggregate Operating Costs for Primary Oil Recovery Operations, 1994-1997

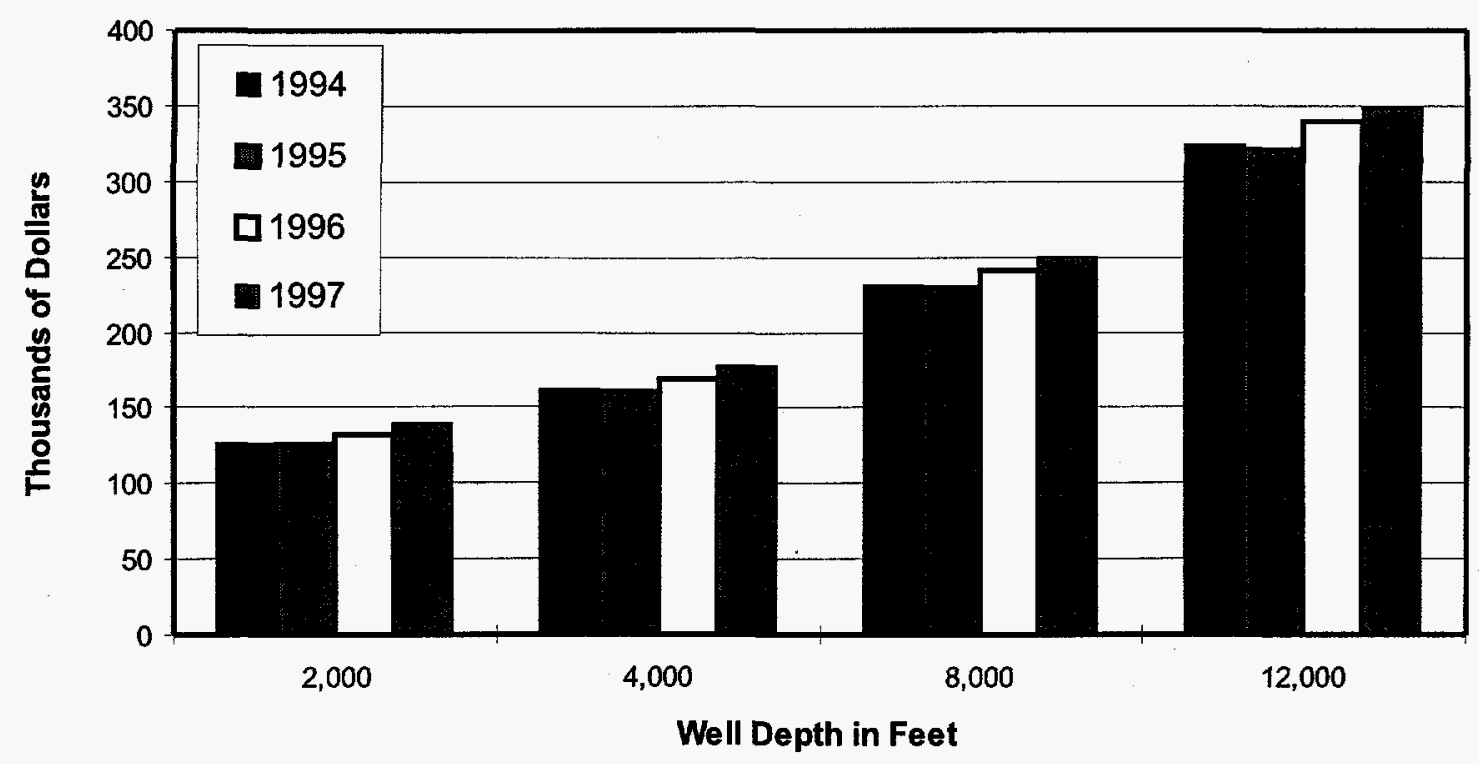

Source: Energy Information Administration, Office of Oil and Gas.

Figure 11. Annual Operating Costs for Secondary Oil Recovery in west Texas, 1994-1997

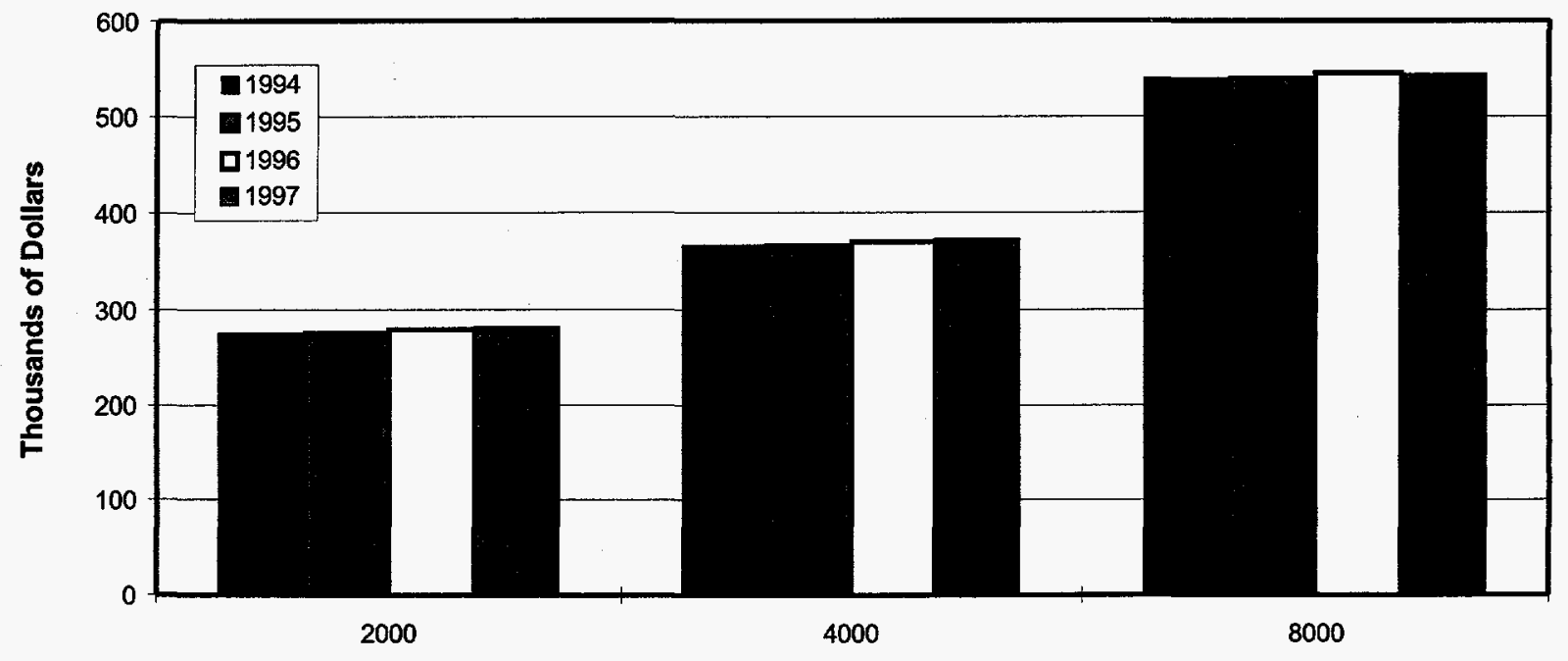

Depth in Feet

Source: Energy Information Administration, Office of Oil and Gas. 
Table 4. Summary of Direct Annual Operating Costs and Composite Indices for Secondary Oil Recovery Operations in West Texas

\begin{tabular}{|c|c|c|c|c|c|}
\hline \multirow[b]{2}{*}{ Component } & \multicolumn{4}{|c|}{ Index $(1976=100)$} & \multirow{2}{*}{$\begin{array}{c}1997^{*} \\
\text { Cost } \\
\text { (dollars) }\end{array}$} \\
\hline & 1994 & 1995 & 1996 & 1997 & \\
\hline & \multicolumn{5}{|c|}{ 2,000-Foot Wells } \\
\hline 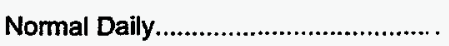 & 277.8 & 280.2 & 285.9 & 290.8 & 179,400 \\
\hline Surface Repair....................................... & 226.3 & 223.5 & 225.8 & 228.1 & 49,500 \\
\hline Subsurface Repair.............................. . & 200.4 & 201.1 & 196.0 & 202.9 & 55,200 \\
\hline \multirow[t]{2}{*}{ 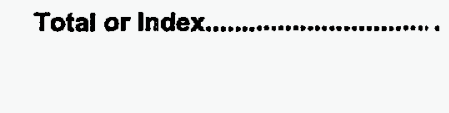 } & 248.6 & 249.6 & 252.0 & 256.9 & 284,100 \\
\hline & \multicolumn{5}{|c|}{ 4,000-Foot Wells } \\
\hline 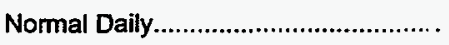 & 274.9 & 277.0 & 281.3 & 285.4 & 215,800 \\
\hline 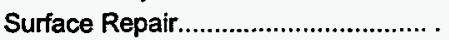 & 232.7 & 228.3 & 230.3 & 232.4 & 79,700 \\
\hline Subsurface Repair................................. . & 196.6 & 197.5 & 193.4 & 199.8 & 81,500 \\
\hline \multirow[t]{2}{*}{ 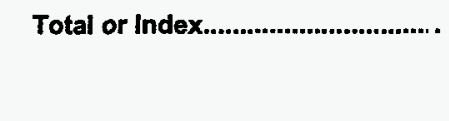 } & 244.1 & 244.4 & 245.9 & 250.2 & 377,000 \\
\hline & \multicolumn{5}{|c|}{ 8,000-Foot Wells } \\
\hline 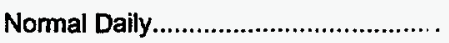 & 287.0 & 289.0 & 292.3 & 295.8 & 310,600 \\
\hline Surface Repair...................................... . & 236.6 & 232.4 & 234.2 & 236.1 & 89,700 \\
\hline Subsurface Repair................................ . & 202.8 & 203.6 & 199.9 & 206.0 & 155,300 \\
\hline 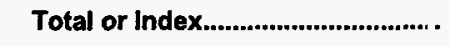 & 249.2 & 249.7 & 250.3 & 254.4 & 555,600 \\
\hline Aggregate Average............................ & 247.5 & 248.0 & 249.3 & 253.7 & 405,600 \\
\hline
\end{tabular}

- Preliminary

Note: Reported average or aggregate average indices are indices of the average costs. They are not an average of the index values.

Source: Energy Information Administration, Office of Oil and Gas

Table 5. Summary of Direct Annual Operating Costs and Composite Indices per Platform--Gulf of Mexico (10,500-Foot True Vertical Depth Wells)

\begin{tabular}{|c|c|c|c|c|c|}
\hline \multirow[b]{2}{*}{ Water Depth } & \multicolumn{4}{|c|}{ Index $(1976=100)$} & \multirow{2}{*}{$\begin{array}{c}1997^{*} \\
\text { Cosit } \\
\text { (dollars) }\end{array}$} \\
\hline & 1994 & 1995 & 1996 & 1997 & \\
\hline & \multicolumn{5}{|c|}{ 12-Slot Platforms } \\
\hline 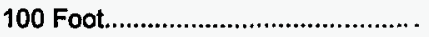 & 242.7 & 252.0 & 258.5 & 258.5 & $3,178,000$ \\
\hline 300 Foot & 238.0 & 247.1 & 253.6 & 252.7 & $3,318,600$ \\
\hline \multirow[t]{2}{*}{ Average or Index.......................... } & 241.7 & 251.0 & 257.6 & 257.1 & $3,248,300$ \\
\hline & \multicolumn{5}{|c|}{ 18-Slot Platforms } \\
\hline 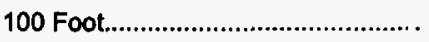 & 234.6 & 244.2 & 251.8 & 246.9 & $3,818,100$ \\
\hline 300 Foot & 227.1 & 236.5 & 244.2 & 239.0 & $3,882,300$ \\
\hline 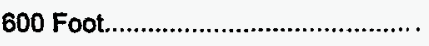 & 222.9 & 233.8 & 240.7 & 231.4 & $4,270,680$ \\
\hline Average or Index........................... . & 230.5 & 240.5 & 248.0 & 241.3 & $3,990,360$ \\
\hline Aggregate Average.......................... & 234.4 & 244.2 & 251.3 & 246.7 & $3,693,500$ \\
\hline
\end{tabular}

- Preliminary

Note: Reported average or aggregate average indices are indices of the average costs. They are not an average of the index values.

Source: Energy information Administration, Office of Oil and Gas 
Figure 12. Fuel, Power, and Water Cost Indices for 12,000-Foot Oil Wells in

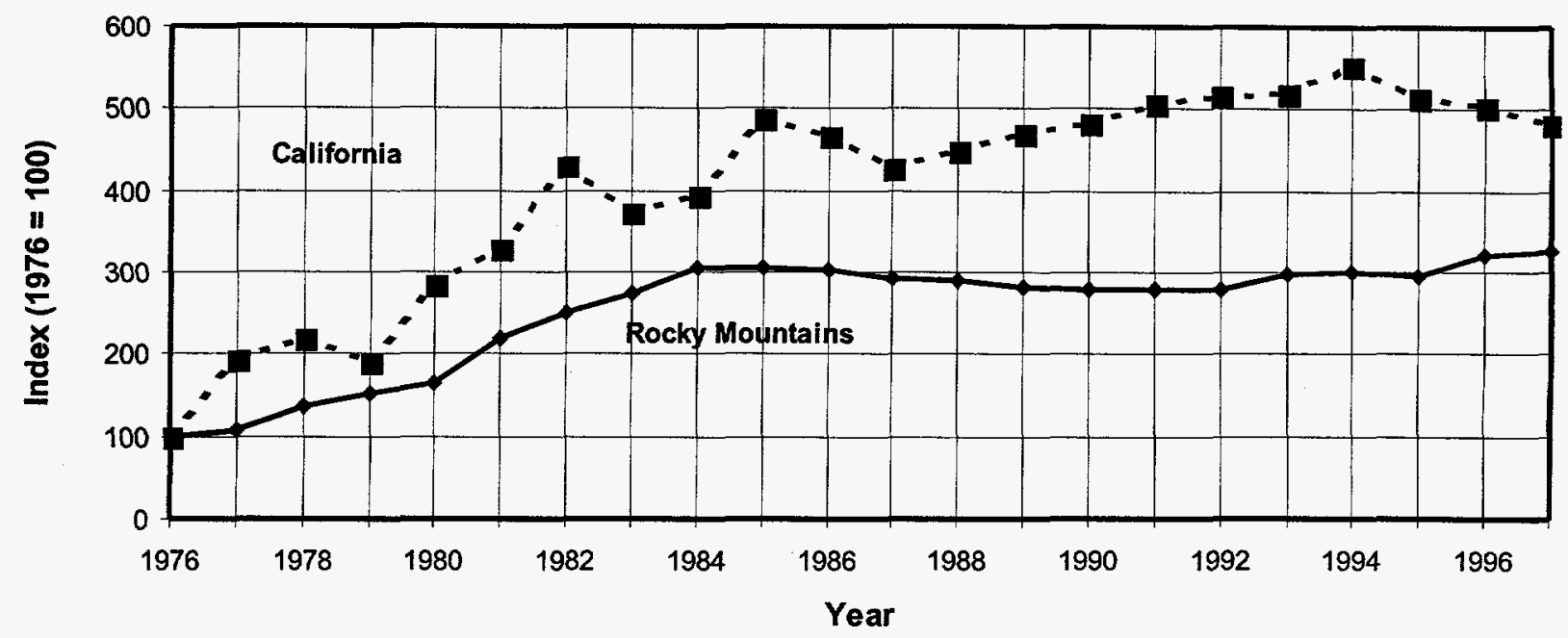

Source: Energy Information Administration, Office of Oil and Gas.

Figure 13. Fuel, Power, and Water Cost Indices for Primary and Secondary Operating Costs

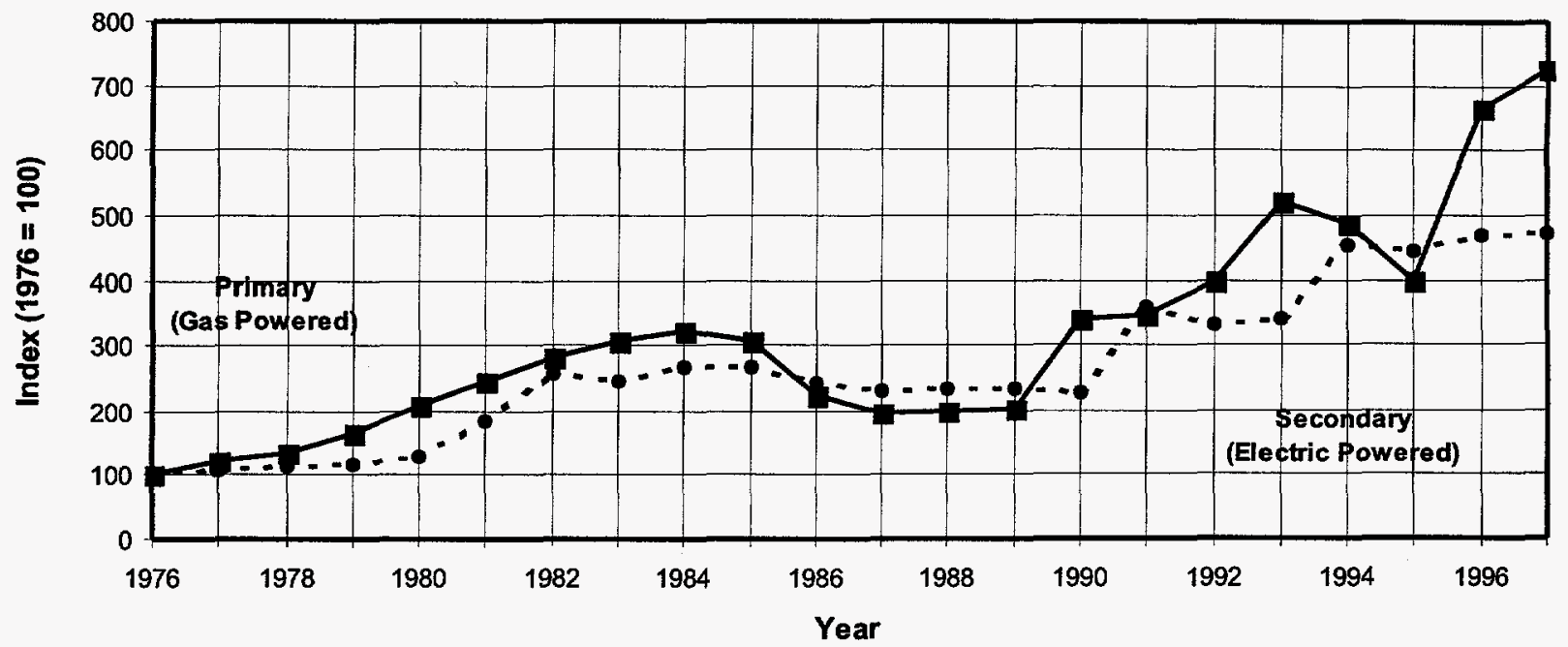

Source: Energy Information Administration, Office of Oil and Gas. 
Figure 14. Annual Gas Well Equipment Costs by Well Depth and Production Rate (1997)

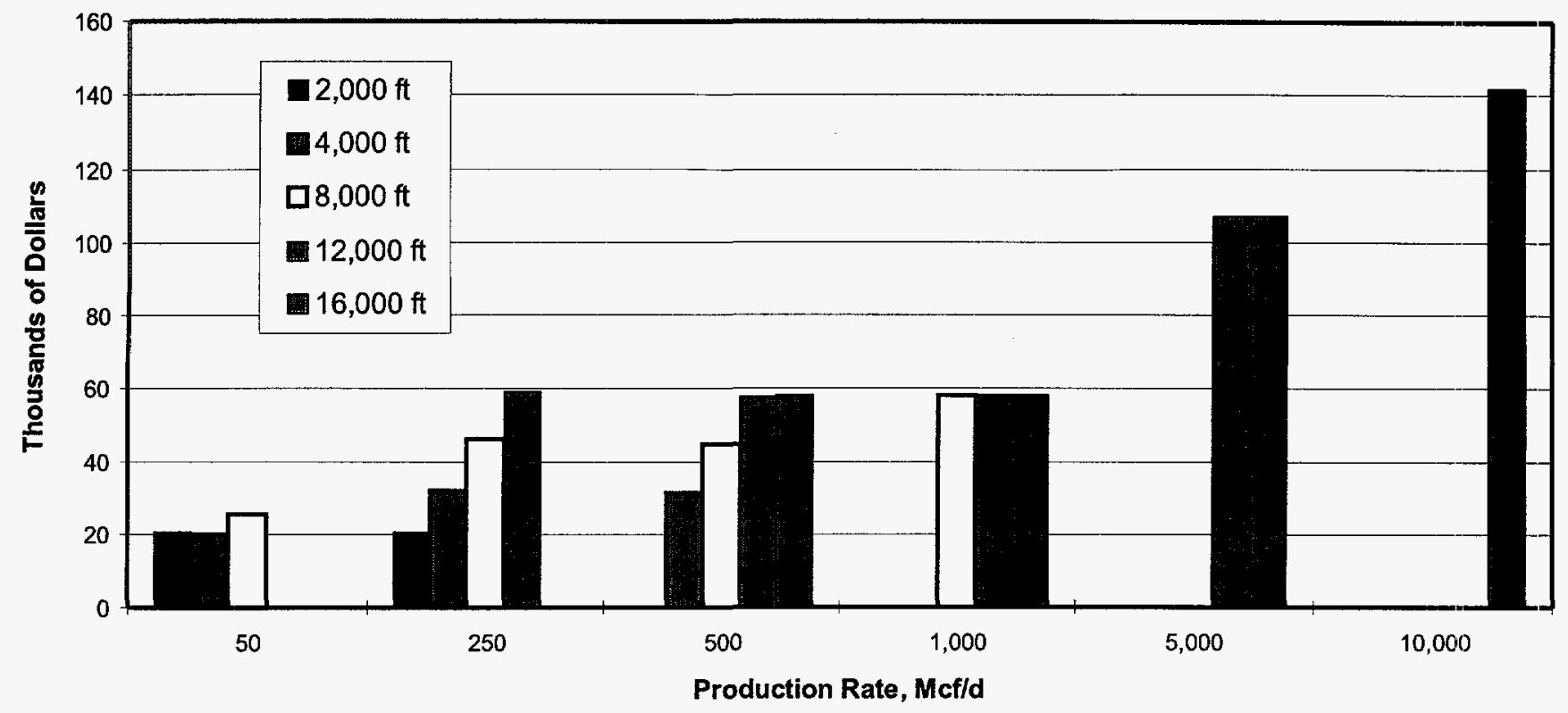

Source: Energy Information Administration, Office of Oil and Gas.

Figure 15. Aggregate Average Equipment Costs for a One-well Gas Lease by Production Rate,

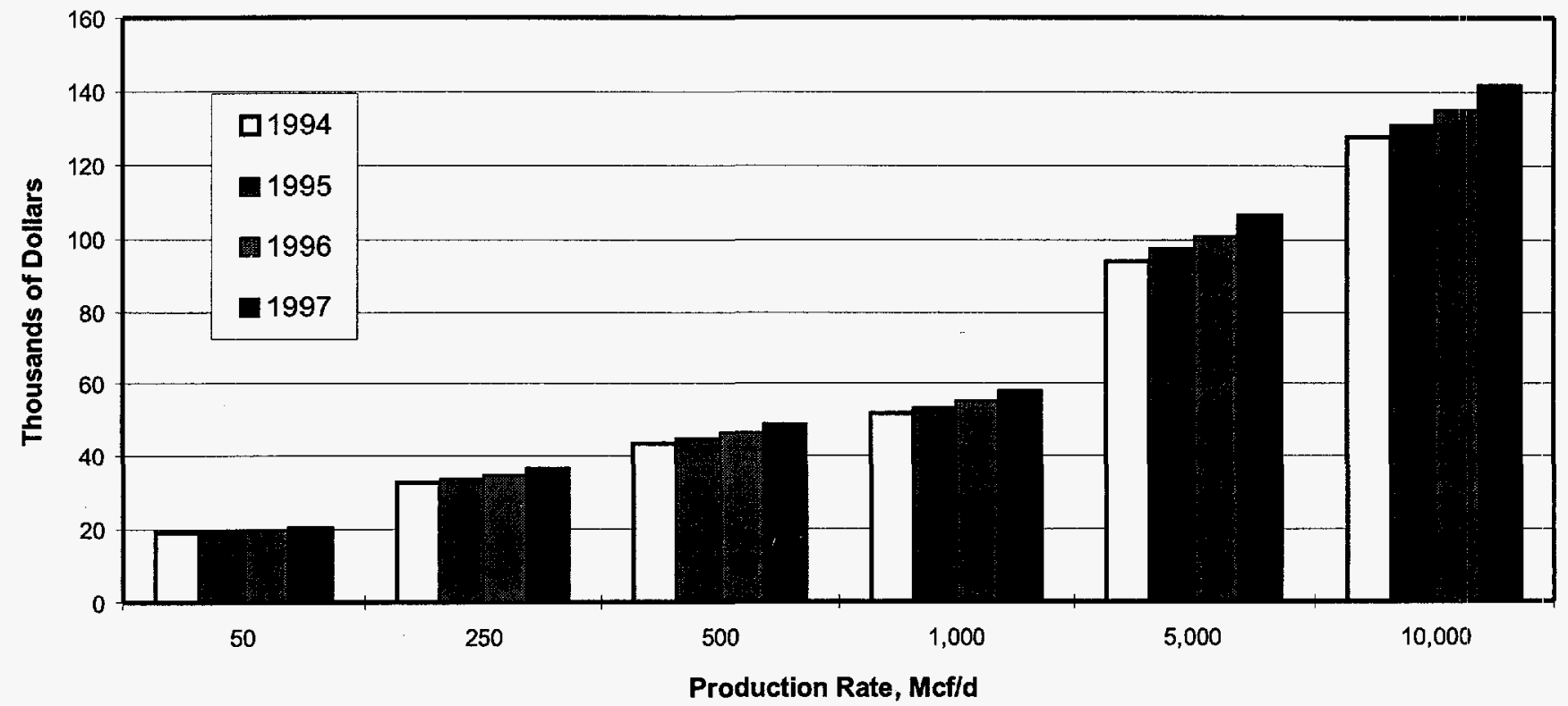

Source: Energy Information Administration, Office of Oil and Gas. 
Table 6. Average Equipment Costs and Indices for Gas Leases Aggregated for All Depths, Areas and Production Rates (One Producing Well)

\begin{tabular}{|c|c|c|c|c|}
\hline \multicolumn{4}{|c|}{ Index $(1976=100)$} & \multirow{2}{*}{$\begin{array}{c}1997^{*} \\
\text { Cost } \\
\text { (dollars) }\end{array}$} \\
\hline 1994 & 1995 & 1996 & 1997 & \\
\hline 184.2 & 190.2 & 196.6 & 206.0 & 48,200 \\
\hline
\end{tabular}

Aggregate average for

all Production Rates................................

- Preliminary

Note: Reported average or aggregate average indices are indices of the average costs. They are not an average of the index values.

Source: Energy Information Administration, Office of Oil and Gas

Table 7. Summary of Gas Lease Equipment Costs and Composite Indices for One Well Producing 50 Thousand Cubic Feet per Day

\begin{tabular}{|c|c|c|c|c|c|}
\hline \multirow[b]{2}{*}{ Area } & \multicolumn{4}{|c|}{ Index $(1976=100)$} & \multirow{2}{*}{$\begin{array}{c}1997^{\star} \\
\text { Cost } \\
\text { (dollars) }\end{array}$} \\
\hline & 1994 & 1995 & 1996 & 1997 & \\
\hline & \multicolumn{5}{|c|}{ 2,000-Foot Wells } \\
\hline 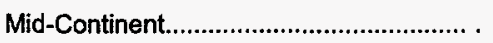 & 181.2 & 186.1 & 194.1 & 203.0 & 20,500 \\
\hline 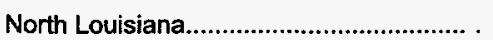 & 172.4 & 178.1 & 185.7 & 195.2 & 20,500 \\
\hline 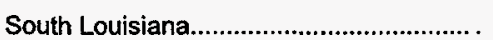 & 172.4 & 178.1 & 185.7 & 195.2 & 20,500 \\
\hline 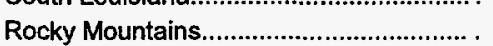 & 177.7 & 182.1 & 189.3 & 197.3 & 22,100 \\
\hline 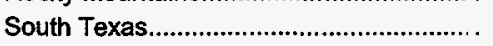 & 174.8 & 179.6 & 186.4 & 192.2 & 19,800 \\
\hline 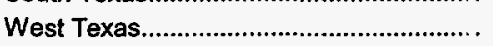 & 180.2 & 185.1 & 168.3 & 173.3 & 17,500 \\
\hline \multirow[t]{2}{*}{ 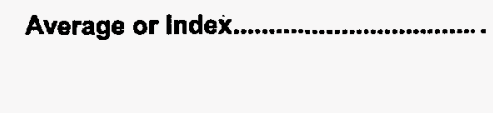 } & 175.2 & 181.0 & 183.8 & 192.4 & 20,200 \\
\hline & \multicolumn{5}{|c|}{ 4,000-Foot Wells } \\
\hline 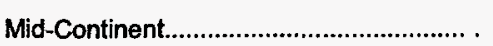 & 181.2 & 186.1 & 194.1 & 203.0 & 20,500 \\
\hline 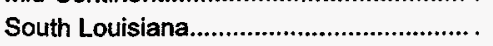 & 172.4 & 178.1 & 185.7 & 195.2 & 20,500 \\
\hline 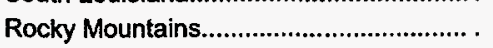 & 177.7 & 182.1 & 189.3 & 197.3 & 22,100 \\
\hline 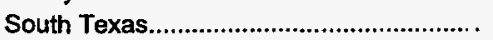 & 174.8 & 179.6 & 186.4 & 192.2 & 19,800 \\
\hline 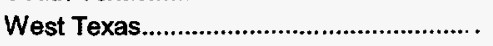 & 180.2 & 185.1 & 168.3 & 173.3 & 17,500 \\
\hline \multirow[t]{2}{*}{ 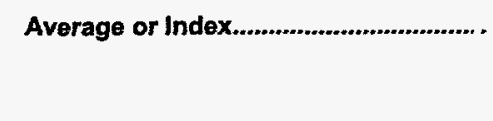 } & 177.9 & 182.7 & 185.6 & 193.3 & 20,100 \\
\hline & \multicolumn{5}{|c|}{ 8,000-Foot Wells } \\
\hline 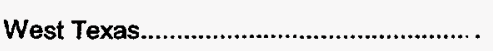 & 189.3 & 196.9 & 187.8 & 193.9 & 25,400 \\
\hline \multicolumn{6}{|l|}{ Aggregate } \\
\hline Average for Production Rate.................. . & 177.6 & 183.2 & 185.0 & 192.5 & 20,600 \\
\hline
\end{tabular}

* Preliminary

Note: Reported average or aggregate average indices are indices of the average costs. They are not an average of the index values.

Source: Energy Information Administration, Office of Oil and Gas 
Table 8. Summary of Gas Lease Equipment Costs and Composite Indices for One Well Producing 250 Thousand Cubic Feet per Day

\begin{tabular}{|c|c|c|c|c|c|}
\hline \multirow[b]{2}{*}{ Area } & \multicolumn{4}{|c|}{ Index $(1976=100)$} & \multirow{2}{*}{$\begin{array}{c}1997^{\star} \\
\text { Cost } \\
\text { (dollars) }\end{array}$} \\
\hline & 1994 & 1995 & 1996 & 1997 & \\
\hline & \multicolumn{5}{|c|}{ 2,000-Foot Wells } \\
\hline Mid-Continent................................................. & 187.9 & 192.5 & 200.0 & 209.3 & 22,400 \\
\hline North Louisiana & 172.4 & 178.1 & 185.7 & 195.2 & 20,500 \\
\hline 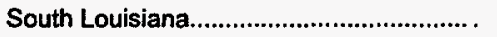 & 172.4 & 178.1 & 185.7 & 195.2 & 20,500 \\
\hline 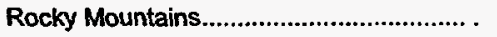 & 177.7 & 182.1 & 189.3 & 197.3 & 22,100 \\
\hline 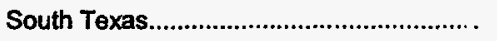 & 174.8 & $\uparrow 79.6$ & 186.4 & 192.2 & 19,800 \\
\hline 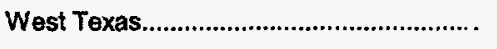 & 180.2 & 185.1 & 168.3 & 173.3 & 17,500 \\
\hline \multirow[t]{2}{*}{ Average or Index................................... } & 176.4 & 182.1 & 184.9 & 193.4 & 20,500 \\
\hline & \multicolumn{5}{|c|}{4,000 -Foot Wells } \\
\hline 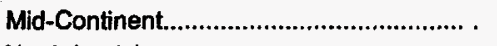 & 193.3 & 202.2 & 207.5 & 217.9 & 29,200 \\
\hline North Louisiana............................................. & 189.2 & 197.8 & 205.0 & 215.1 & 29,900 \\
\hline South Louisiana............................................ & 186.3 & 194.2 & 203.6 & 213.7 & 29,700 \\
\hline Rocky Mountains......................................... & 173.6 & 177.9 & 184.7 & 194.5 & 45,700 \\
\hline 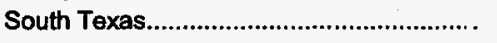 & 187.7 & 194.2 & 202.9 & 209.4 & 28,900 \\
\hline West Texas.................................................... & 194.0 & 200.0 & 191.8 & 197.8 & 26,500 \\
\hline \multirow[t]{2}{*}{ Average or Index..................................... } & 186.3 & 192.8 & 198.0 & 207.2 & 31,700 \\
\hline & \multicolumn{5}{|c|}{$8,000-$ Foot Welis } \\
\hline Mid-Continent................................................. & 180.9 & 185.2 & 192.6 & 203.0 & 46,700 \\
\hline 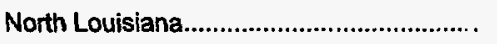 & 173.1 & 177.7 & 185.3 & 195.8 & 46,600 \\
\hline 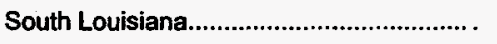 & 173.9 & 178.6 & 187.4 & 197.9 & 47,100 \\
\hline 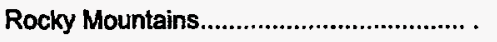 & 147.0 & 149.3 & 155.1 & 162.5 & 48,100 \\
\hline 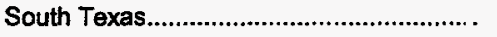 & 175.4 & 179.2 & 187.7 & 194.5 & 45,900 \\
\hline 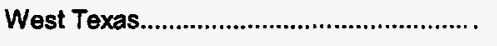 & 179.1 & 182.6 & 181.3 & 188.3 & 43,300 \\
\hline \multirow[t]{2}{*}{ 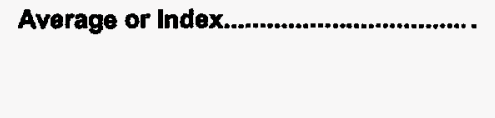 } & 170.2 & 174.3 & 180.4 & 189.0 & 46,300 \\
\hline & \multicolumn{5}{|c|}{ 12,000-Foot Wells } \\
\hline Mid-Continent........................................... . & 207.4 & 214.1 & 225.0 & 236.7 & 60,600 \\
\hline Rocky Mountains........................................ . & 171.8 & 176.6 & 184.0 & 192.9 & 60,200 \\
\hline 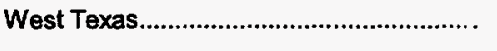 & 204.7 & 210.9 & 212.5 & 221.5 & 56,700 \\
\hline Average or Index.................................... & 192.7 & 198.5 & 205.5 & 215.3 & 59,200 \\
\hline \multicolumn{6}{|l|}{ Aggregate } \\
\hline Average for Production Rate.................. . & 180.3 & 185.2 & 190.7 & 200.0 & 36,600 \\
\hline
\end{tabular}

- Preliminary

Note: Reported average or aggregate average indices are indices of the average costs. They are not an average of the index values.

Source: Energy Information Administration, Office of Oil and Gas 
Table 9. Summary of Gas Lease Equipment Costs and Composite Indices for One Well Producing 500 Thousand Cubic Feet per Day

\begin{tabular}{|c|c|c|c|c|c|}
\hline \multirow[b]{2}{*}{ Area } & \multicolumn{4}{|c|}{ Index $(1976=100)$} & \multirow{2}{*}{$\begin{array}{c}1997^{\star} \\
\text { Cost } \\
\text { (dollars) }\end{array}$} \\
\hline & 1994 & 1995 & 1996 & 1997 & \\
\hline & \multicolumn{5}{|c|}{ 4,000-Foot Wells } \\
\hline Mid-Continent & 189.8 & 199.2 & 204.7 & 215.0 & 27,300 \\
\hline North Louisiana a & 174.0 & 179.8 & 188.5 & 197.1 & 20,500 \\
\hline 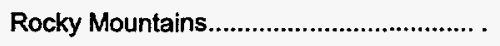 & 144.4 & 147.2 & 152.4 & 159.8 & 45,700 \\
\hline \multirow[t]{2}{*}{ Average or Index } & 161.6 & 166.9 & 172.7 & 181.4 & 31,200 \\
\hline & \multicolumn{5}{|c|}{ 8,000-Foot Wells } \\
\hline 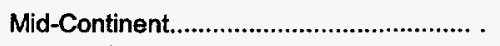 & 175.9 & 179.4 & 186.8 & 195.2 & 44,500 \\
\hline 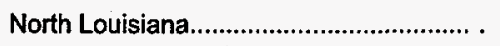 & 168.9 & 173.2 & 182.1 & 192.3 & 45,200 \\
\hline 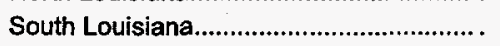 & 168.9 & 173.2 & 182.1 & 192.3 & 45,200 \\
\hline 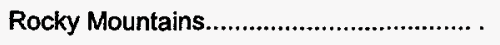 & 144.8 & 147.6 & 153.1 & 160.4 & 46,200 \\
\hline 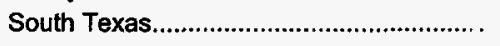 & 170.0 & 173.8 & 182.4 & 189.7 & 44,200 \\
\hline 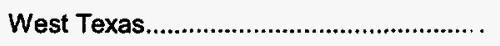 & 173.2 & 176.8 & 175.4 & 182.5 & 41,600 \\
\hline \multirow[t]{2}{*}{ 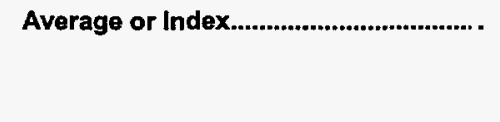 } & 166.4 & 169.7 & 176.3 & 184.6 & 44,500 \\
\hline & \multicolumn{5}{|c|}{ 12,000-Foot Wells } \\
\hline 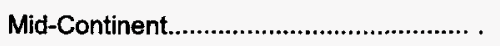 & 206.4 & 212.9 & 224.1 & 235.7 & 58,700 \\
\hline 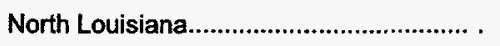 & 199.2 & 206.3 & 217.2 & 228.9 & 58,600 \\
\hline 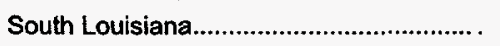 & 199.2 & 206.3 & 217.2 & 228.9 & 58,600 \\
\hline Rocky Mountains. & 170.4 & 175.7 & 182.9 & 191.8 & 58,300 \\
\hline 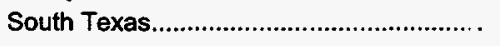 & 200.4 & 207.1 & 217.7 & 226.8 & 57,600 \\
\hline 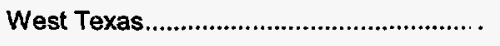 & 204.0 & 210.4 & 212.0 & 220.9 & 55,000 \\
\hline \multirow[t]{2}{*}{ 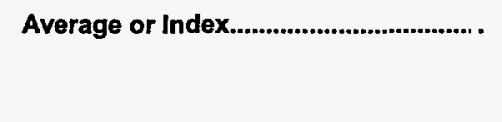 } & 196.2 & 202.3 & 211.1 & 221.5 & 57,800 \\
\hline & \multicolumn{5}{|c|}{ 16,000-Foot Wells } \\
\hline 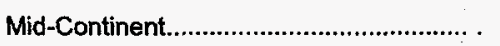 & 174.8 & 179.9 & 187.2 & 197.0 & 58,700 \\
\hline South Louisiana & 199.2 & 206.3 & 217.2 & 228.9 & 58,600 \\
\hline 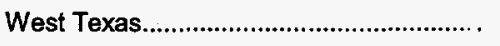 & 172.1 & 177.5 & 184.2 & 192.6 & 57,400 \\
\hline Average or Index & 181.3 & 187.0 & 195.1 & 204.9 & 58,200 \\
\hline \multicolumn{6}{|l|}{ Aggregate } \\
\hline Average for Production Rate................. . & 178.7 & 184.0 & 191.4 & 200.8 & 49,000 \\
\hline
\end{tabular}

* Preliminary

Note: Reported average or aggregate average indices are indices of the average costs. They are not an average of the index values.

Source: Energy Information Administration, Office of Oil and Gas 
Table 10. Summary of Gas Lease Equipment Costs and Composite Indices for One Well Producing 1 Million Cubic Feet per Day

\begin{tabular}{|c|c|c|c|c|c|}
\hline \multirow[b]{2}{*}{ Area } & \multicolumn{4}{|c|}{ Index $(1976=100)$} & \multirow{2}{*}{$\begin{array}{c}1997 * \\
\text { Cost } \\
\text { (dollars) }\end{array}$} \\
\hline & 1994 & 1995 & 1996 & 1997 & \\
\hline & \multicolumn{5}{|c|}{8,000 Foot Wells } \\
\hline 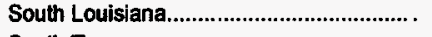 & 170.4 & 175.7 & 182.9 & 192.8 & 58,600 \\
\hline 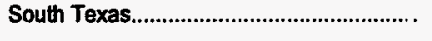 & 171.8 & 177.1 & 184.1 & 191.7 & 57,700 \\
\hline \multirow[t]{2}{*}{ 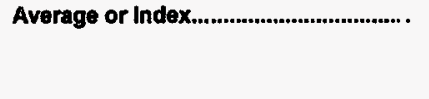 } & 171.0 & 176.2 & 183.2 & 192.1 & 58,200 \\
\hline & \multicolumn{5}{|c|}{ 12,000-Foot Wells } \\
\hline 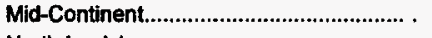 & 174.8 & 179.9 & 187.2 & 197.0 & 58,700 \\
\hline 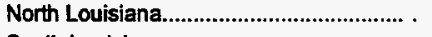 & 170.4 & 175.7 & 182.9 & 192.8 & 58,600 \\
\hline 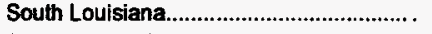 & 170.4 & 175.7 & 182.9 & 192.8 & 58,600 \\
\hline 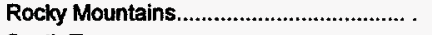 & 170.4 & 175.7 & 182.9 & 191.8 & 58,300 \\
\hline 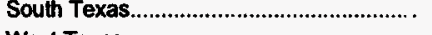 & 171.2 & 176.5 & 183.4 & 191.1 & 57,700 \\
\hline West Texas & 172.1 & 177.5 & 184.2 & 192.6 & $\mathbf{5 7 , 4 0 0}$ \\
\hline \multirow[t]{2}{*}{ 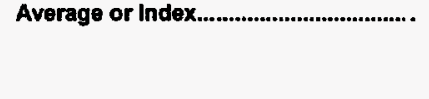 } & 171.5 & 176.5 & 183.8 & 192.7 & 58,200 \\
\hline & \multicolumn{5}{|c|}{ 16,000-Foot Wells } \\
\hline 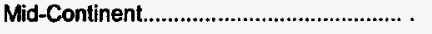 & 174.8 & 179.9 & 187.2 & 197.0 & 58,700 \\
\hline 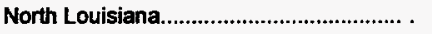 & 170.4 & 175.7 & 182.9 & 192.8 & 58,600 \\
\hline 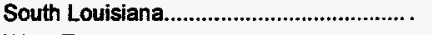 & 170.4 & 175.7 & 182.9 & 192.8 & 58,600 \\
\hline 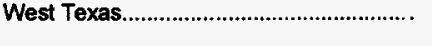 & 172.1 & 177.5 & 184.2 & 192.6 & 57,400 \\
\hline Average or Index & 172.1 & 177.1 & 184.4 & 193.7 & 58,300 \\
\hline \multicolumn{6}{|l|}{ Aggregate } \\
\hline Average for Production Rate.................... . & 171.5 & 176.5 & 183.8 & 192.7 & 58,200 \\
\hline
\end{tabular}

- Preliminary

Note: Reported average or aggregate average indices are indices of the average costs. They are not an average of the index values.

Source: Energy Information Administration, Office of Oil and Gas

Table 11. Summary of Gas Lease Equipment Costs and Composite Indices for One Well Producing 5 Million Cubic Feet per Day

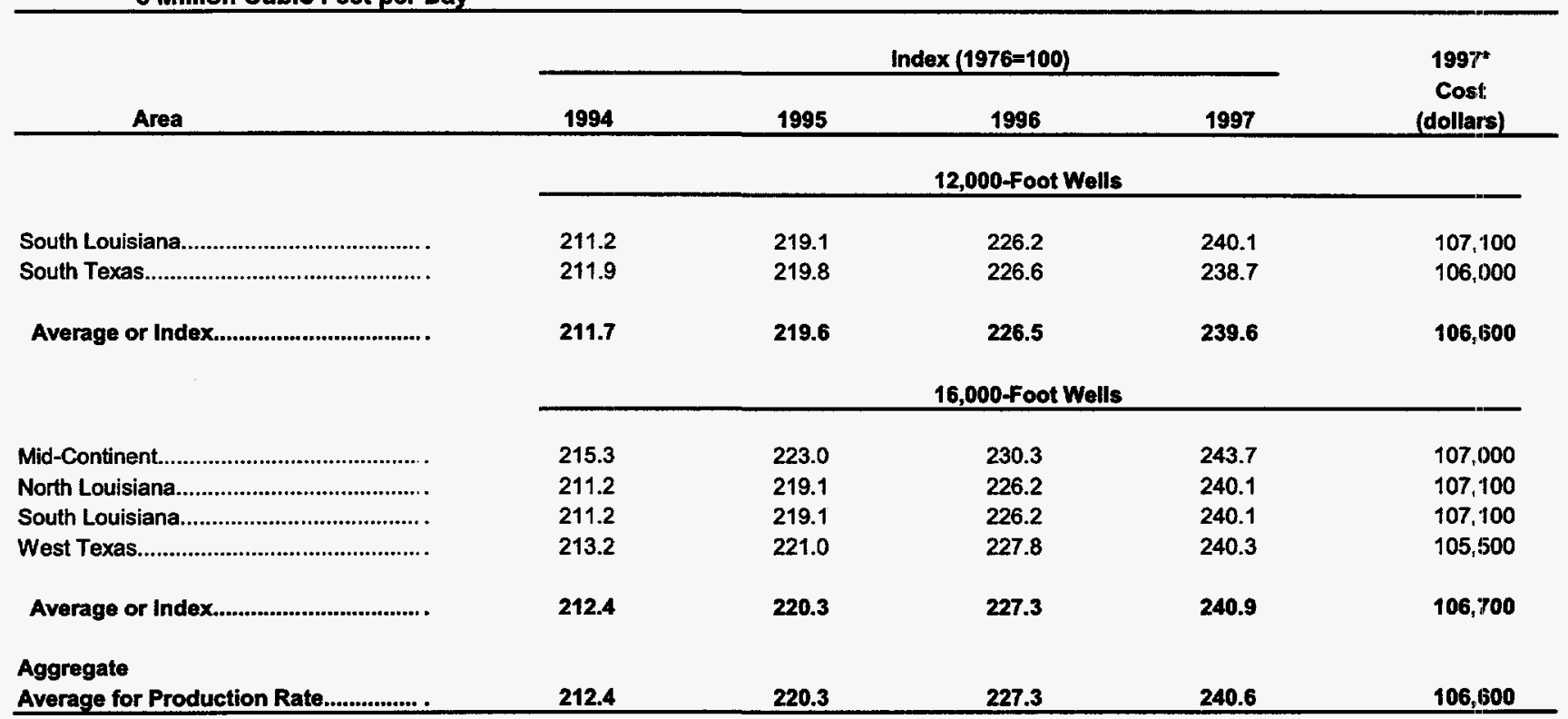

- Preliminary

Note: Reported average or aggregate average indices are indices of the average costs. They are not an average of the index values.

Source: Energy Information Administration, Office of Oil and Gas 
Table 12. Summary of Gas Lease Equipment Costs and Composite Indices for One Well Producing 10 Million Cubic Feet per Day

\begin{tabular}{|c|c|c|c|c|c|}
\hline \multirow[b]{2}{*}{ Area } & \multicolumn{4}{|c|}{ Index $(1976=100)$} & \multirow{2}{*}{$\begin{array}{c}1997^{*} \\
\text { Cost } \\
\text { (dollars) }\end{array}$} \\
\hline & 1994 & 1995 & 1996 & 1997 & \\
\hline North Louisiana................................. . & 215.0 & 221.1 & 227.7 & 239.1 & 141,800 \\
\hline
\end{tabular}

*Preliminary

Note: Reported average or aggregate average indices are indices of the average costs. They are not an average of the index values.

Source: Energy Information Administration, Office of Oil and Gas

Table 13. Summary of Aggregate Average Gas Lease Equipment Costs by Well Depth (1994-1997)

\begin{tabular}{|c|c|c|c|c|}
\hline \multirow{2}{*}{$\begin{array}{l}\text { Well Depth } \\
\text { (feet) }\end{array}$} & \multicolumn{4}{|c|}{ Average Costs (dollars) } \\
\hline & 1994 & 1995 & 1996 & 1997 \\
\hline 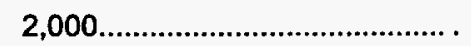 & 18,600 & 19,100 & 19,500 & 20,300 \\
\hline 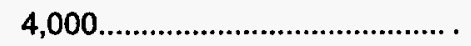 & 24,800 & 25,600 & 26,200 & 27,400 \\
\hline 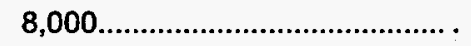 & 41,300 & 42,300 & 43,700 & 45,800 \\
\hline 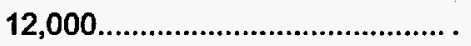 & 56,800 & 58,600 & 60,900 & 63,900 \\
\hline 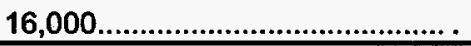 & 72,100 & 74,500 & 77,200 & 81,400 \\
\hline
\end{tabular}

- Preliminary

Source: Energy Information Administration, Office of Oil and Gas 
Table 14. Average Operating Costs and Indices for Gas Leases Aggregated for All Depths, Areas and Production Rates (One Producing Well)

\begin{tabular}{|c|c|c|c|c|c|}
\hline \multirow[b]{2}{*}{ Area } & \multicolumn{4}{|c|}{ Index $(1976=100)$} & \multirow{2}{*}{$\begin{array}{c}1997^{*} \\
\text { Cost } \\
\text { (dollars) }\end{array}$} \\
\hline & 1994 & 1995 & 1996 & 1997 & \\
\hline \multicolumn{6}{|l|}{ Aggregate Average for } \\
\hline all Production Rates............................... & 216.0 & 218.9 & 222.6 & 235.8 & 25,000 \\
\hline
\end{tabular}

- Preliminary

Note: Reported average or aggregate average indices are indices of the average costs. They are not an average of the index values.

Source: Energy Information Administration, Office of Oil and Gas

Table 15. Summary of Gas Lease Operating Costs and Composite Indices for One Well Producing 50 Thousand Cubic Feet per Day

\begin{tabular}{|c|c|c|c|c|c|}
\hline \multirow[b]{2}{*}{ Area } & \multicolumn{4}{|c|}{ Index $(1976=100)$} & \multirow{2}{*}{$\begin{array}{c}\text { 19s7* } \\
\text { Cost } \\
\text { (dollars) }\end{array}$} \\
\hline & 1994 & 1995 & 1996 & 1997 & \\
\hline & \multicolumn{5}{|c|}{ 2,000-Foot Wells } \\
\hline 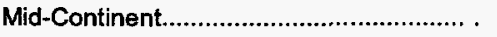 & 270.6 & 276.5 & 282.4 & 302.9 & 10,300 \\
\hline North Louisiana............................................. . & 222.0 & 229.3 & 229.3 & 251.2 & 10,300 \\
\hline South Louisiana.......................................... & 224.4 & 231.7 & 231.7 & 251.2 & 10,300 \\
\hline Rocky Mountains........................................ . & 220.8 & 222.9 & 225.0 & 241.7 & 11,600 \\
\hline 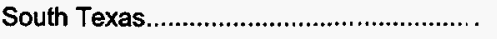 & 248.7 & 256.4 & 253.8 & 256.4 & 10,000 \\
\hline 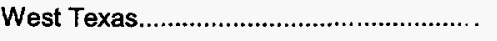 & 250.0 & 252.9 & 250.0 & 252.9 & 8,600 \\
\hline \multirow[t]{2}{*}{ Average or Index.................................... . } & 235.0 & 240.0 & 240.0 & 255.0 & 10,200 \\
\hline & \multicolumn{5}{|c|}{ 4,000-Foot Wells } \\
\hline 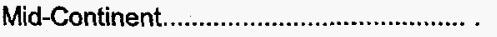 & 250.0 & 254.8 & 259.5 & 278.6 & 11,700 \\
\hline 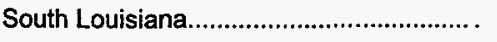 & 225.5 & 231.9 & 234.0 & 253.2 & 11,900 \\
\hline Rocky Mountains & 216.1 & 221.4 & 221.4 & 239.3 & 13,400 \\
\hline 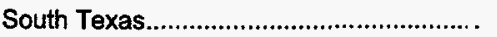 & 246.7 & 251.1 & 251.1 & 255.6 & 11,500 \\
\hline 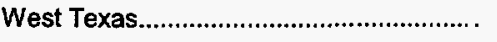 & 248.8 & 251.2 & 253.7 & 258.5 & 10,600 \\
\hline \multirow[t]{2}{*}{ Average or Index.................................... } & 237.0 & 241.3 & 243.5 & 256.5 & 11,800 \\
\hline & \multicolumn{5}{|c|}{8,000 -Foot Wells } \\
\hline 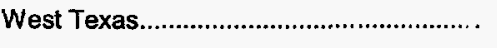 & 225.9 & 229.3 & 227.6 & 237.9 & 13,800 \\
\hline \multicolumn{6}{|l|}{ Aggregate } \\
\hline Average for Production Rate.................. . & 234.1 & 238.6 & 240.9 & 254.5 & 11,200 \\
\hline
\end{tabular}

* Preliminary

Note: Reported average or aggregate average indices are indices of the average costs. They are not an average of the index values.

Source: Energy Information Administration, Office of Oil and Gas 
Table 16. Summary of Gas Lease Operating Costs and Composite Indices for One Well Producing 250 Thousand Cubic Feet per Day

\begin{tabular}{|c|c|c|c|c|c|}
\hline \multirow[b]{2}{*}{ Area } & \multicolumn{4}{|c|}{ Index $(1976=100)$} & \multirow{2}{*}{$\begin{array}{c}1997^{\star} \\
\text { Cost } \\
\text { (dollars) }\end{array}$} \\
\hline & 1994 & 1995 & 1996 & 1997 & \\
\hline & \multicolumn{5}{|c|}{ 2,000-Foot Wells } \\
\hline 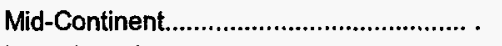 & 261.7 & 266.0 & 268.1 & 291.5 & 13,700 \\
\hline 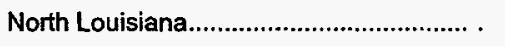 & 218.4 & 224.5 & 224.5 & 242.9 & 11,900 \\
\hline 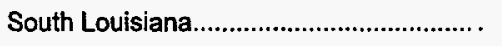 & 220.4 & 226.5 & 226.5 & 246.9 & 12,100 \\
\hline 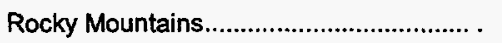 & 217.9 & 219.6 & 221.4 & 239.3 & 13,400 \\
\hline 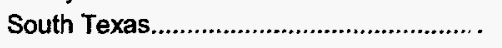 & 240.4 & 246.8 & 244.7 & 251.1 & 11,800 \\
\hline 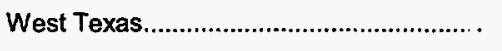 & 240.5 & 242.9 & 240.5 & 247.6 & 10.400 \\
\hline \multirow[t]{2}{*}{ 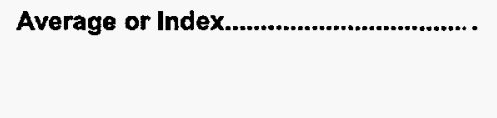 } & 233.3 & 239.6 & 239.6 & 254.2 & 12,200 \\
\hline & \multicolumn{5}{|c|}{ 4,000-Foot Wells } \\
\hline 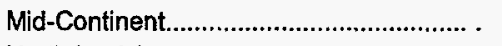 & 247.0 & 251.5 & 254.5 & 274.2 & 18,100 \\
\hline 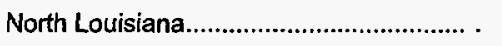 & 217.9 & 222.4 & 226.9 & 244.8 & 16,400 \\
\hline 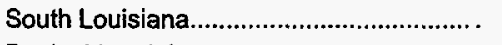 & 222.4 & 228.4 & 229.9 & 249.3 & 16,700 \\
\hline 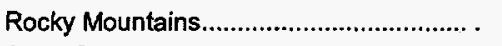 & 216.1 & 219.4 & 220.4 & 236.6 & 22,000 \\
\hline South Texas & 232.3 & 235.4 & 235.4 & 241.5 & 15,700 \\
\hline 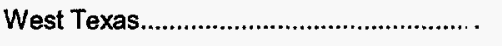 & 229.5 & 232.8 & 232.8 & 239.3 & 14,600 \\
\hline \multirow[t]{2}{*}{ 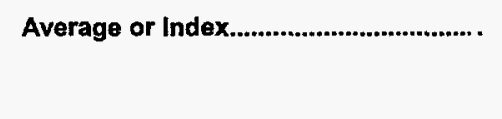 } & 225.7 & 230.0 & 231.4 & 247.1 & 17,300 \\
\hline & \multicolumn{5}{|c|}{8,000 -Foot Wells } \\
\hline 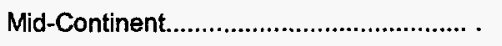 & 243.4 & 246.2 & 248.1 & 267.9 & 28,400 \\
\hline 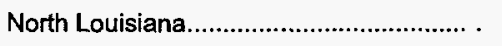 & 214.4 & 218.6 & 220.3 & 239.0 & 28,200 \\
\hline 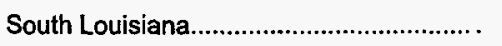 & 216.1 & 220.3 & 222.0 & 240.7 & 28,400 \\
\hline 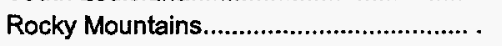 & 215.2 & 216.0 & 220.0 & 236.0 & 29,500 \\
\hline 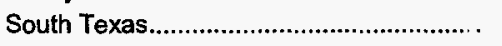 & 224.3 & 227.8 & 227.8 & 233.9 & 26,900 \\
\hline 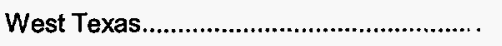 & 225.5 & 228.3 & 227.4 & 236.8 & 25,100 \\
\hline \multirow[t]{2}{*}{ Average or Index } & 221.7 & 225.2 & 227.0 & 241.7 & 27,800 \\
\hline & \multicolumn{5}{|c|}{ 12,000-Foot Wells } \\
\hline 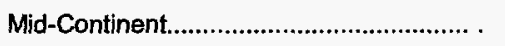 & 236.8 & 239.8 & 241.4 & 260.2 & 34,600 \\
\hline 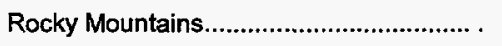 & 217.0 & 219.0 & 221.6 & 237.9 & 36,400 \\
\hline 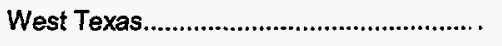 & 220.0 & 223.7 & 223.0 & 231.9 & 31,300 \\
\hline 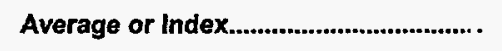 & 225.0 & 227.9 & 228.6 & 243.6 & 34,100 \\
\hline \multicolumn{6}{|l|}{ Aggregate } \\
\hline Average for Production Rate................. . & 224.1 & 227.6 & 228.7 & 243.7 & 21,200 \\
\hline
\end{tabular}

* Preliminary

Note: Reported average or aggregate average indices are indices of the average costs. They are not an average of the index values.

Source: Energy Information Administration, Office of Oil and Gas 
Table 17. Summary of Gas Lease Operating Costs and Composite Indices for One Well Producing 500 Thousand Cubic Feet per Day

\begin{tabular}{|c|c|c|c|c|c|}
\hline \multirow[b]{2}{*}{ Area } & \multicolumn{4}{|c|}{ Index (1976=100) } & \multirow{2}{*}{$\begin{array}{c}1997^{*} \\
\text { Cosit } \\
\text { (dollars) }\end{array}$} \\
\hline & 1994 & 1995 & 1996 & 1997 & \\
\hline & \multicolumn{5}{|c|}{ 4,000-Foot Wells } \\
\hline 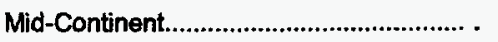 & 263.3 & 266.7 & 270.0 & 291.7 & 17,500 \\
\hline North Louisiana......................................... . & 215.5 & 219.7 & 222.5 & 245.1 & 17,400 \\
\hline Rocky Mountains.......................................... . & 211.6 & 213.7 & 217.9 & 234.7 & 22,300 \\
\hline \multirow[t]{2}{*}{ Average or Index.................................. } & 228.0 & 230.7 & 234.7 & 254.7 & 19,100 \\
\hline & \multicolumn{5}{|c|}{8,000 -Foot Wells } \\
\hline Mid-Continent.............................................. & 257.8 & 261.4 & 262.7 & 285.5 & 23,700 \\
\hline North Louisiana............................................ . & 209.6 & 214.4 & 217.3 & 237.5 & 24,700 \\
\hline South Louisiana........................................ & 212.5 & 217.3 & 220.2 & 241.3 & 25,100 \\
\hline Rocky Mountains.................................... . & 214.3 & 216.2 & 221.9 & 239.0 & 25,100 \\
\hline 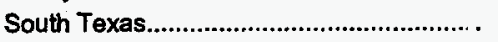 & 192.7 & 195.5 & 197.3 & 196.4 & 21,600 \\
\hline 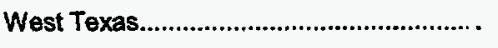 & 227.7 & 228.9 & 230.1 & 234.9 & 19,500 \\
\hline \multirow[t]{2}{*}{ Average or Index.................................. } & 217.3 & 220.4 & 223.5 & 237.8 & 23,300 \\
\hline & \multicolumn{5}{|c|}{ 12,000-Foot Wells } \\
\hline Mid-Continent............................................. & 248.5 & 252.4 & 254.4 & 274.8 & 28,300 \\
\hline North Louisiana....................................... . & 205.1 & 211.0 & 213.6 & 232.2 & 27,400 \\
\hline South Louisiana & 215.3 & 219.5 & 222.9 & 241.5 & 28,500 \\
\hline Rocky Mountains...................................... . & 215.7 & 217.3 & 222.0 & 238.6 & 30,300 \\
\hline 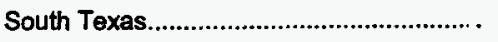 & 229.8 & 233.3 & 235.1 & 237.7 & 27,100 \\
\hline 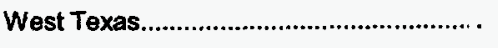 & 219.0 & 222.9 & 223.8 & 227.6 & 23,900 \\
\hline \multirow[t]{2}{*}{ 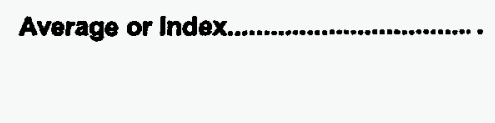 } & 221.9 & 225.4 & 228.1 & 242.1 & 27,600 \\
\hline & \multicolumn{5}{|c|}{16,000 -Foot Wells } \\
\hline Mid-Continent & 237.3 & 239.0 & 243.2 & 262.7 & 31,000 \\
\hline South Louisiana...................................... & 209.8 & 215.2 & 217.4 & 234.8 & 31,000 \\
\hline 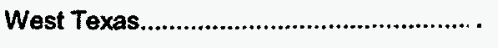 & 217.5 & 220.8 & 222.5 & 227.5 & 27,300 \\
\hline 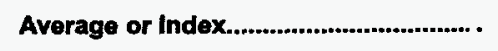 & 222.0 & 225.2 & 228.5 & 242.3 & $\mathbf{2 9 , 8 0 0}$ \\
\hline \multicolumn{6}{|l|}{ Aggregate } \\
\hline Average for Production Rate.................. & 220.2 & 224.0 & 226.9 & 241.3 & 25,100 \\
\hline
\end{tabular}

- Preliminary

Note: Reported average or aggregate average indices are indices of the average costs. They are not an average of the index values.

Source: Energy Information Administration, Office of Oil and Gas 
Table 18. Summary of Gas Lease Operating Costs and Composite Indices for One Well Producing 1 Million Cubic Feet per Day

\begin{tabular}{|c|c|c|c|c|c|}
\hline \multirow[b]{2}{*}{ Area } & \multicolumn{4}{|c|}{ Index $(1976=100)$} & \multirow{2}{*}{$\begin{array}{c}1997^{\star} \\
\text { Cost } \\
\text { (dollars) }\end{array}$} \\
\hline & 1994 & 1995 & 1996 & 1997 & \\
\hline & \multicolumn{5}{|c|}{ 8,000-Foot Wells } \\
\hline 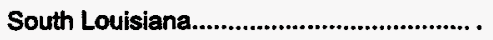 & 212.2 & 216.0 & 220.6 & 242.0 & 31,700 \\
\hline 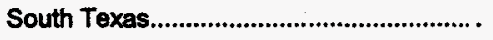 & 248.8 & 250.4 & 252.8 & 255.1 & 32,400 \\
\hline \multirow[t]{2}{*}{ Average or Index } & 230.2 & 233.3 & 236.4 & 248.8 & 32,100 \\
\hline & \multicolumn{5}{|c|}{ 12,000-Foot Wells } \\
\hline 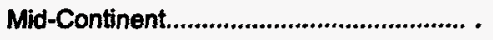 & 247.4 & 249.6 & 254.1 & 275.2 & 36,600 \\
\hline 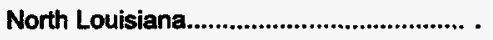 & 203.3 & 207.2 & 211.8 & 231.4 & 35,400 \\
\hline 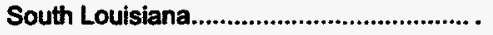 & 213.7 & 217.6 & 222.2 & 241.8 & 37,000 \\
\hline 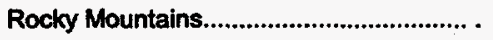 & 219.9 & 221.2 & 226.3 & 243.6 & 38,000 \\
\hline 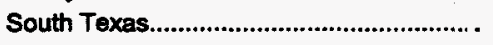 & 207.4 & 210.1 & 212.8 & 214.8 & 32,000 \\
\hline 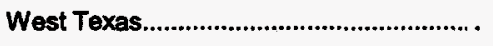 & 216.9 & 219.1 & 221.3 & 226.5 & 30,800 \\
\hline \multirow[t]{2}{*}{ 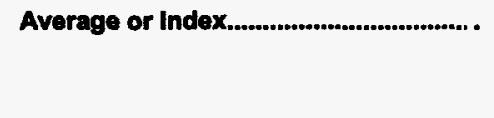 } & 217.0 & 219.7 & 223.8 & 238.1 & 35,000 \\
\hline & \multicolumn{5}{|c|}{ 16,000-Foot Wells } \\
\hline 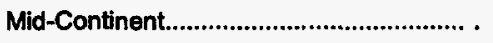 & 240.5 & 242.6 & 247.3 & 266.9 & 39,500 \\
\hline 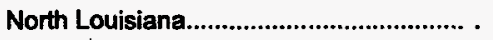 & 205.2 & 208.7 & 212.8 & 230.8 & 39,700 \\
\hline 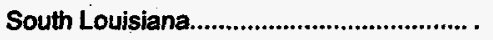 & 207.0 & 210.5 & 214.5 & 232.6 & 40,000 \\
\hline 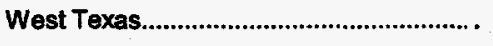 & 217.3 & 220.7 & 222.0 & 227.3 & 34,100 \\
\hline Average or Index & 216.1 & 219.3 & 223.0 & 237.9 & 38,300 \\
\hline \multicolumn{6}{|l|}{ Aggregate } \\
\hline Average for Production Rate.................. . & 219.6 & 222.3 & 226.4 & 240.5 & 35,600 \\
\hline
\end{tabular}

- Preliminary

Note; Reported average or aggregate average indices are indices of the average costs. They are not an average of the index values.

Source: Energy Information Administration, Office of Oil and Gas 
Table 19. Summary of Gas Lease Operating Costs and Composite Indices for One Well Producing 5 Million Cubic Feet per Day

\begin{tabular}{|c|c|c|c|c|c|}
\hline \multirow[b]{2}{*}{ Area } & \multicolumn{4}{|c|}{ Index $(1976=100)$} & \multirow{2}{*}{$\begin{array}{c}19917^{*} \\
\text { Cost } \\
\text { (dollars) }\end{array}$} \\
\hline & 1994 & 1995 & 1996 & 1997 & \\
\hline & \multicolumn{5}{|c|}{ 12,000-Foot Wells } \\
\hline 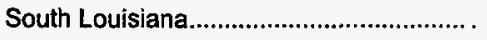 & 204.9 & 207.6 & 216.7 & 229.9 & 33,100 \\
\hline 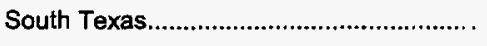 & 195.8 & 199.4 & 206.0 & 210.2 & 34,900 \\
\hline \multirow[t]{2}{*}{ Average or Index } & 200.0 & 203.2 & 211.0 & 219.4 & 34,000 \\
\hline & \multicolumn{5}{|c|}{16,000 -Foot Wells } \\
\hline Mid-Continent.............................................. . & 187.2 & 189.8 & 197.4 & 209.7 & 41,100 \\
\hline 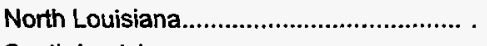 & 185.7 & 188.1 & 195.7 & 205.7 & 43,200 \\
\hline 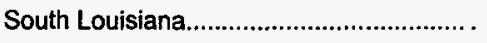 & 185.6 & 188.5 & 195.2 & 204.8 & 42,800 \\
\hline 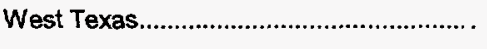 & 180.0 & 183.0 & 189.0 & 194.5 & 38,900 \\
\hline 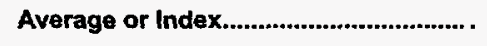 & 184.3 & 187.3 & 194.1 & 203.4 & 41,500 \\
\hline \multicolumn{6}{|l|}{ Aggregate } \\
\hline Average for Production Rate.................. . & 188.3 & 191.5 & 198.4 & 207.4 & 39,000 \\
\hline
\end{tabular}

- Preliminary

Note: Reported average or aggregate average indices are indices of the average costs. They are not an average of the index values.

Source: Energy Information Administration, Office of Oil and Gas

Table 20. Summary of Gas Lease Operating Costs and Composite Indices for One Well Producing 10 Million Cubic Feet per Day

\begin{tabular}{|c|c|c|c|c|c|}
\hline \multirow[b]{2}{*}{ Area } & \multicolumn{4}{|c|}{ Index $(1976=100)$} & \multirow{2}{*}{$\begin{array}{c}1997^{*} \\
\text { Cost } \\
\text { (dollairs) }\end{array}$} \\
\hline & 1994 & 1995 & 1996 & 1997 & \\
\hline & \multicolumn{5}{|c|}{16,000 -Foot Wells } \\
\hline 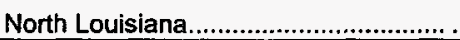 & 180.1 & 180.8 & 189.2 & 197.9 & 56,800 \\
\hline
\end{tabular}

\section{*Preliminary}

Note: This is the only area in which a 16,000 -foot well producing 10 MMcf per day is reported.

Source: Energy Information Administration, Office of Oil and Gas

Table 21. Summary of Aggregate Average Gas Lease Operating Cost, by Well Depth (1994-97)

\begin{tabular}{|c|c|c|c|c|}
\hline \multirow{2}{*}{$\begin{array}{c}\text { Well Depth } \\
\text { (feet) }\end{array}$} & \multicolumn{4}{|c|}{ Average Cost, Dollars } \\
\hline & 1994 & 1995 & 1996 & $1997^{*}$ \\
\hline $2,000 \ldots \ldots \ldots \ldots \ldots$ & 10,300 & 10,500 & 10,500 & 11,200 \\
\hline $4,000 \ldots \ldots \ldots \ldots \ldots \ldots \ldots \ldots$ & 14,300 & 14,600 & 14,700 & 15,700 \\
\hline $8,000 \ldots \ldots \ldots \ldots$ & 23,600 & 23,900 & 24,100 & 25,600 \\
\hline 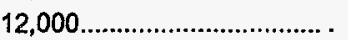 & 29,400 & 29,800 & 30,300 & 32,100 \\
\hline 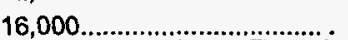 & 35,300 & 35,700 & 36,700 & 38,800 \\
\hline
\end{tabular}

- Preliminary

Source: Energy Information Administration, Office of Oil and Gas 
Figure 16. Aggregate Average Annual Gas Well Operating Costs for a One-Well Gas Lease by

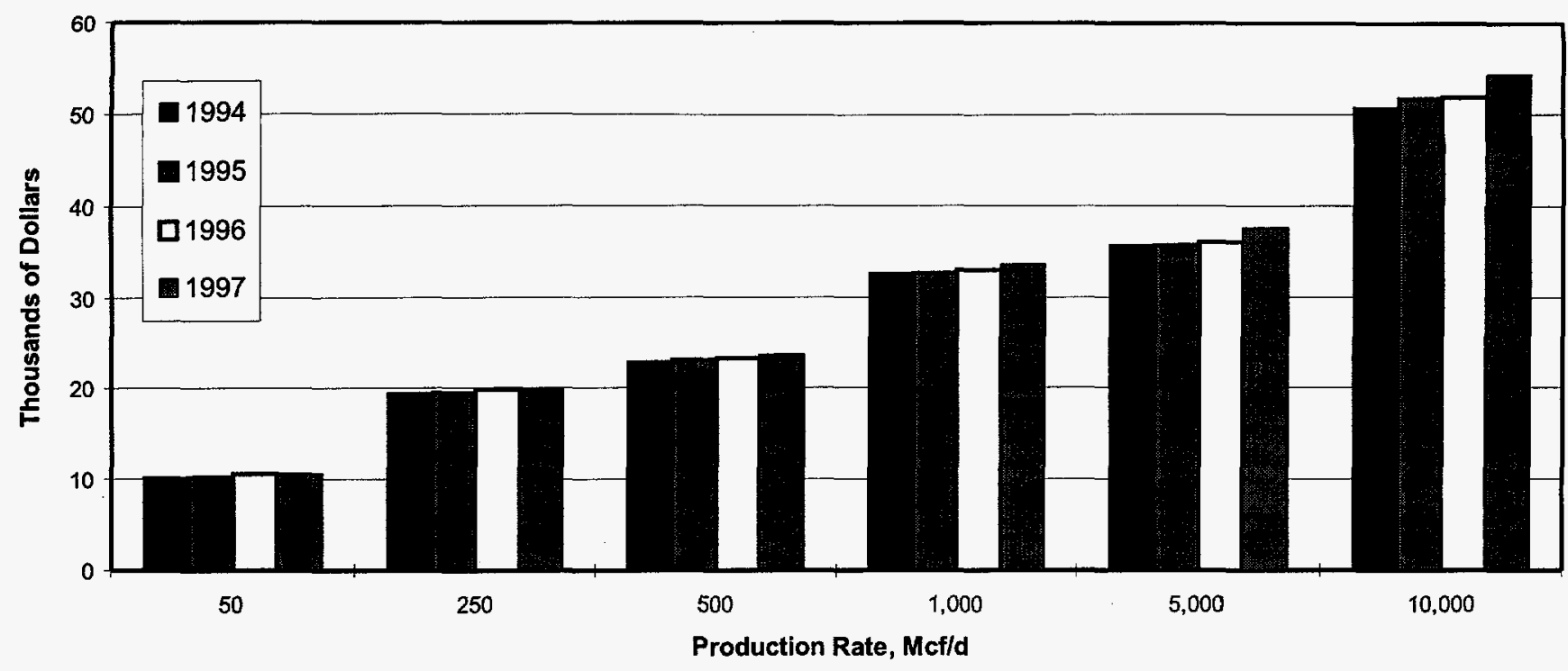

Source: Energy Information Administration, Office of Oil and Gas.

Figure 17. Annual Gas Well Operating Costs by Depth and Production Rate, 1997

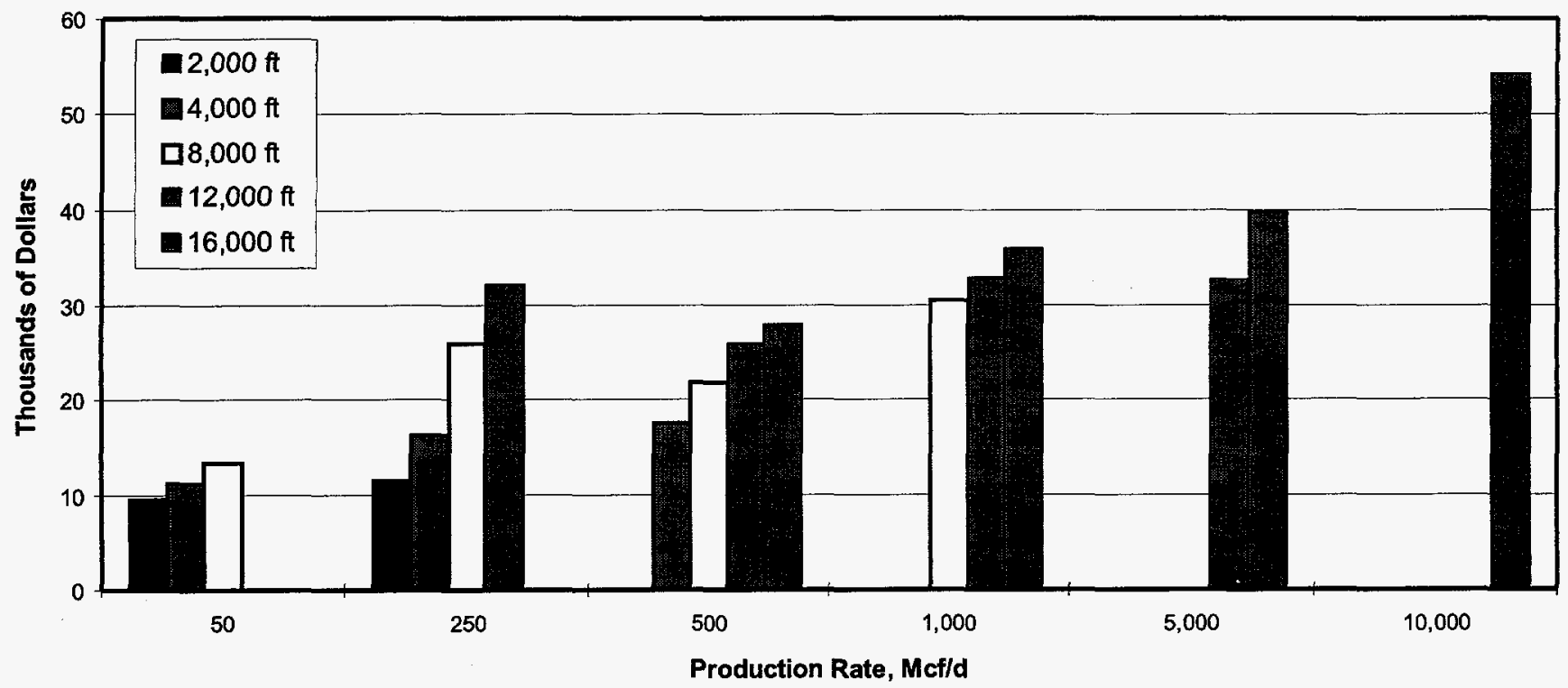

Source: Energy Information Administration, Office of Oil and Gas. 



\section{Indexing Review}

\section{Technological and Data Changes}

The uniform oil lease equipment design adopted in 1976 was the basic criterion for oil lease equipment cost estimates. Revisions have been made to stay current with engineering and competitive practices. Individual component prices were combined into one price for a group of equipment, as necessary, to assure confidentiality of prices. Appendix Tables A15 through A18 contain detailed equipment lists of representative wells in west Texas for each depth, reflecting all changes made to date.

Standardization of the data used has evolved during the past 19 years. Improved methods for measuring various contractor costs were used and applied to previous estimates. The gas lease equipment designs were made in 1980 and the equipment and operating components were priced back through 1976. There have been no recent design changes for gas equipment. A typical design is shown in Appendix Table H11, which contains a list of equipment for a 12,000-foot gas well producing $1 \mathrm{MMcf}$ per day in west Texas.

Estimated preliminary costs for the prior report were revised to reflect new data. Some of these changes and factors were:
- New projections of Joint Association Survey (JAS) data for west Texas were made to estimate 1997 drilling costs.

- Regional wellhead gas prices for 1994-1997 are from the latest edition of the EIA Natural Gas Annual (DOE/EIA-0131 96). These 1997 prices are estimated.

\section{Primary Oil Recovery}

Leases for oil wells were assumed to consist of 10 wells producing by artificial lift into a centrally located tank battery. The depths of all wells on the leases were $2,000,4,000,8,000$, or 12,000 feet.

Costs were determined for new equipment capable of producing 200 barrels of liquid per day per well for onshore primary operations. Tubing costs were included for information only. Note that care must be exercised when combining these equipment costs with drilling costs to obtain total lease development and equipment costs, because most drilling cost estimates include tubing costs. The artificial lift selected was dependent upon the type of lift found to be dominant for each depth in each region. The two types of prime movers considered were electric motors and natural gas engines. Table 22 details the type of lift and prime mover used in each region and depth.

Table 22. Type of Artificial Lift and Prime Mover Used for Each Depth and Region

\begin{tabular}{|c|c|c|c|c|}
\hline Region & $\begin{array}{c}\text { Type of } \\
\text { Lift }\end{array}$ & $\begin{array}{l}\text { Prime } \\
\text { Mover }\end{array}$ & $\begin{array}{c}\text { Type of } \\
\text { Lift }\end{array}$ & $\begin{array}{l}\text { Prime } \\
\text { Mover }\end{array}$ \\
\hline & \multicolumn{2}{|c|}{ 2,000-Foot Wells } & \multicolumn{2}{|c|}{ 4,000-Foot Wells } \\
\hline California............................ . & Rod & Motor & Rod & Motor \\
\hline 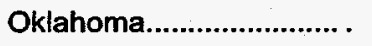 & Rod & Engine & Rod & Engine \\
\hline South Louisiana................ . & Rod & Engine & Gas & Engine \\
\hline South Texas...................... & Rod & Engine & Gas & Engine \\
\hline West Texas........................ & Rod & Engine & Rod & Engine \\
\hline \multirow[t]{2}{*}{ Rocky Mountains.............. } & Rod & Motor & Rod & Motor \\
\hline & \multicolumn{2}{|c|}{ 8,000-Foot Wells } & \multicolumn{2}{|c|}{ 12,000-Foot Wells } \\
\hline 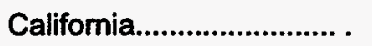 & Hydraulic & Motor & Hydraulic & Motor \\
\hline 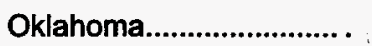 & Hydraulic & Engine & Hydraulic & Engine \\
\hline South Louisiana................ & Gas & Engine & Hydraulic & Engine \\
\hline South Texas...................... . & Gas & Engine & Hydraulic & Engine \\
\hline West Texas........................ & Rod & Engine & Hydraulic & Engine \\
\hline Rocky Mountains............. . & Rod & Motor & Hydraulic & Motor \\
\hline
\end{tabular}

Source: Energy Information Administration, Office of Oil and Gas. 
Annual operating costs were estimated for daily production rates of 100 barrels of liquid per day per well for each depth in each region of operation.

\section{Secondary Oil Recovery}

Costs for secondary oil recovery in west Texas were calculated for wells producing from depths of 2,000, 4,000, and 8,000 feet. Each lease had 10 producing wells, 11 injection wells, and 1 disposal well. Additional costs included those for water supply wells, water storage tanks, injection plant, filtering systems, and injection lines. Equipment was designed to handle 350 barrels of liquid per day per producing well. Gas engines used in primary operations were replaced by electric motors for secondary oil recovery. Some equipment for primary oil production was replaced with larger equipment to accommodate the increased liquid volumes assumed for secondary recovery production. Increases in operational costs for secondary oil recovery are indicated for the increased liquid lift of 290 barrels of liquid per day per producing well and the water injection system. Additional equipment costs are presented in Appendix Tables A9, A10, and A11, and direct annual operating costs are presented in Tables A12, A13, and A14.

\section{Offshore Gas and Primary Oil Recovery}

Equipment and operating costs for the offshore Gulf of Mexico were estimated for 12- and 18-slot platforms containing one dually completed well in each slot. Maximum crude oil production was assumed to total 11,000 barrels of oil per day from wells on each platform. Maximum associated gas production was assumed to be $40 \mathrm{MMcf}$ cubic feet of gas per day per platform. Note that the balance between gas and oil is weighted more heavily toward gas in offshore operations than in onshore leases. Operating costs were derived for platforms assumed to be 50,100, and 125 miles from shore corresponding to water depths of 100,300 , and 600 feet, respectively. Meals, platform maintenance, helicopter and boat transportation of personnel and supplies, communication costs, insurance costs for platform and production equipment and administrative expenses are included in normal production expenses. Crude oil and natural gas transportation costs to shore were excluded, as were water disposal costs.

\section{Gas Recovery}

Leases for gas wells were assumed to consist of one well producing into an onsite separator with two storage tanks (a lease condensate sales tank and a water storage tank). Line heaters, dehydration units, and methanol injectors were included where needed. It was assumed that any compression or gas treatment would be provided by the furst purchaser. The cost data presented were based on the installation of new equipment and included items needed from the wellhead to the inlet on the meter run for the gas stream and through the tank for the liquid streams. Downhole tubing costs were not included, nor were equipment for disposal of produced water above nominal amounts of water entrained in the gas stream. Gas production rates of $50,250,500,1,000,5,000$, and $10,000 \mathrm{Mcf}$ of gas per day and well depths of 2,000,4,000, $8,000,12,000$, and 16,000 feet were the assumed volume and depth divisions for the cost determinations. These volumes were selected because of different processing equipment requirements for each of these flow rates. Production records were used to determine the average production rate for each depth in each region. The equipment and operating costs for each of these average production rates were then calculated. For a broader view of each flow rate in each region at each depth, the equipment and operating costs of the next higher and/or lower rates are shown. Costs were calculated for equipping gas wells at producing rates of $50 \mathrm{Mcf}$ per day even though a new well coming onstream at this rate may never reach payout. This low rate of flow was selected to identify costs of production from stripper gas wells. Flow rates above $10 \mathrm{MMcf}$ per day usually require custom design of equipment and are not priced in this report.

The depths of $2,000,4,000,8,000$, and 12,000 feet were chosen to be compatible with data provided for oil production. An additional depth of 16,000 feet was added for gas equipment and operations because there was significant gas production from this depth in some regions studied. 


\section{Section I}

Appendices A Through $\mathbf{G}$

Costs and Indices for Domestic Oil Field Equipment and Production Operations 


\section{0}




\section{Appendices A Through G}

\section{Costs and Indices for Domestic Oil Field Equipment and Production Operations}

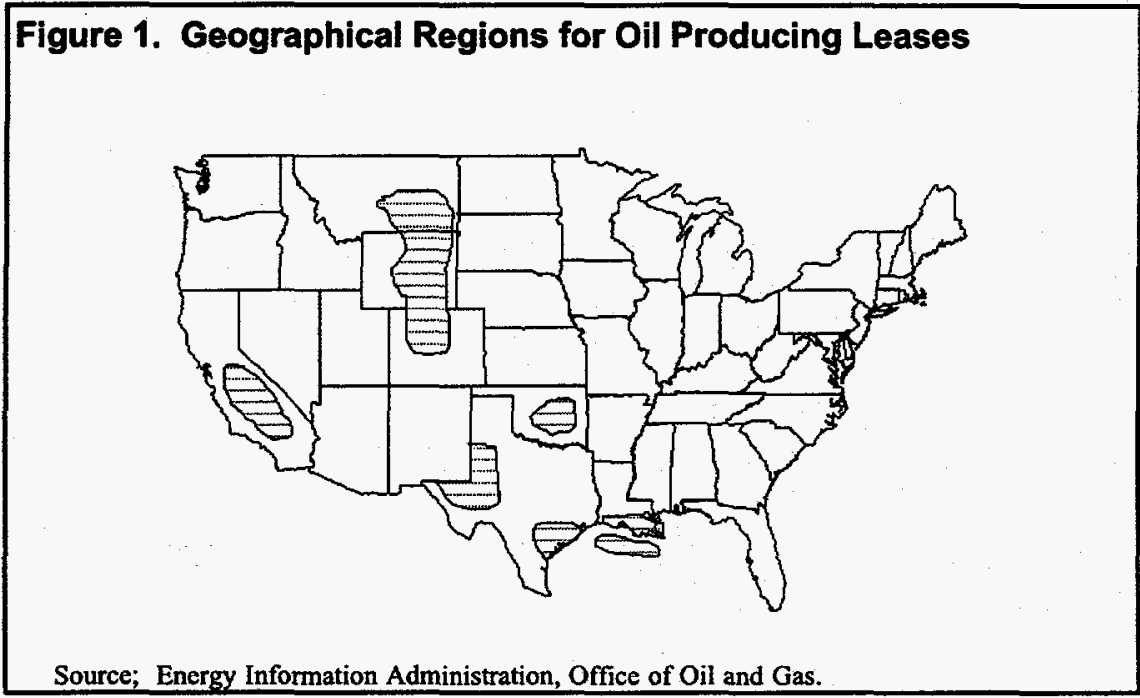

A detailed breakdown of costs and cost indices for 1994 through 1997 is shown in each of the oil lease appendix tables. These tables include both lease equipment costs and direct annual operating costs with their appropriate index numbers. Appendices $A$ through $G$ present the costs and indices for each region and type of operation for oil production.

The tables are arranged by region with each region identified by an alpha character. For example, Tables A1 through A18 are for west Texas. Tables A1 through A4 contain equipment costs and indices for primary production for four depths, beginning with the shallowest depth. Tables A5 through A8 are the annual operating costs and indices by depth for primary operations. Tables A9, A10, and A11 present additional equipment costs required for secondary operations for three depths.Tables A12, A13, and A14 contain annual operating costs by depth for secondary production. Tables A15 through A18 are sample detailed equipment listings by depth for the region. The remaining Tables containing costs and indices for oil leases by region are arranged in similar order. They are: Appendix B--south Texas, Appendix C--south Louisiana, Appendix D--Oklahoma, Appendix E--Wyoming, Appendix F--California, and Appendix G--Gulf of Mexico.

Notes: - 1997 data are preliminary and are marked with a single asterisk $\left({ }^{*}\right)$. - All prior data were revised. - Indices marked with a double asterisk $\left({ }^{* *}\right)$ are composite indices. $\bullet$ Other indices are pure cost. $\bullet$ Entries with (***) are estimated from Joint Association Survey on Drilling Costs data. 
Table A1. Lease Equipment Costs and Indices for Primary Oil Production in West Texas (10 Wells Producing from 2,000 Feet by Rod Lift)

\begin{tabular}{|c|c|c|c|c|c|}
\hline \multirow{2}{*}{ Component } & \multicolumn{4}{|c|}{ Index $(1976=100)$} & \multirow{2}{*}{$\begin{array}{c}1997^{*} \\
\text { Cost: } \\
\text { (dollars) }\end{array}$} \\
\hline & 1994 & 1995 & 1996 & 1997 & \\
\hline \multicolumn{6}{|l|}{ Producing Equipment: } \\
\hline 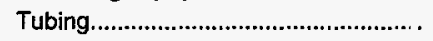 & 110.9 & 155.0 & 157.8 & 175.1 & 62,700 \\
\hline 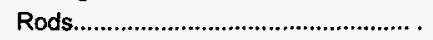 & 122.0 & 122.0 & 122.5 & 120.4 & 23,000 \\
\hline Pumps. & 147.6 & 152.4 & 157.1 & 166.7 & 14,000 \\
\hline Pumping Equipment.............................. . & 175.9 & 188.1 & 199.6 & 233.3 & 305,100 \\
\hline Subtotal or Index $x^{* *} \ldots$ & 157.4 & 173.9 & 182.5 & 208.6 & $404,8: 00$ \\
\hline \multicolumn{6}{|l|}{ Gathering System: } \\
\hline 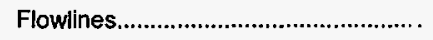 & 251.0 & 254.5 & 260.0 & 268.3 & 38,900 \\
\hline 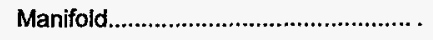 & 263.6 & 266.7 & 273.5 & 290.2 & 38,300 \\
\hline Subtotal or Index & 257.0 & 260.3 & 266.4 & 278.7 & 77,2010 \\
\hline \multicolumn{6}{|l|}{ Lease Equipment: } \\
\hline Producing Separator................................... & 173.4 & 173.4 & 182.8 & 187.5 & 12,000 \\
\hline Test Separator............................................ & 189.1 & 188.1 & 187.1 & 198.0 & 20,000 \\
\hline Heater Treater......................................... & 136.8 & 145.8 & 150.3 & 154.8 & 24,000 \\
\hline Storage Tanks.......................................... & 193.2 & 198.2 & 203.0 & 207.4 & 69,700 \\
\hline 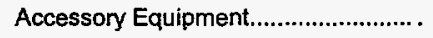 & 212.9 & 220.4 & 227.2 & 227.9 & 33,500 \\
\hline 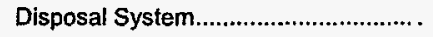 & 189.9 & 193.5 & 196.4 & 204.1 & 84,700 \\
\hline 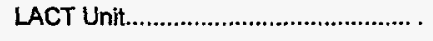 & 174.2 & 171.0 & 171.0 & 171.0 & 15,900 \\
\hline Subtotal or Index ${ }^{* *} \ldots$ & 185.0 & 189.1 & 192.9 & 198.2 & 259,800 \\
\hline Total or Index ${ }^{* *} \ldots$ & 175.5 & 186.3 & 192.9 & 210.2 & 741,800 \\
\hline
\end{tabular}

Table A2. Lease Equipment Costs and Indices for Primary Oil Production in West Texas (10 Wells Producing from 4,000 Feet by Rod Lift)

\begin{tabular}{|c|c|c|c|c|c|}
\hline \multirow{2}{*}{ Component } & \multicolumn{4}{|c|}{ Index $(1976=100)$} & \multirow{2}{*}{$\begin{array}{c}\text { 1997* } \\
\text { Cost' } \\
\text { (dollars) }\end{array}$} \\
\hline & 1994 & 1995 & 1996 & 1997 & \\
\hline \multicolumn{6}{|l|}{ Producing Equipment: } \\
\hline Tubing & 109.2 & 152.9 & 155.8 & 172.6 & 125,300 \\
\hline 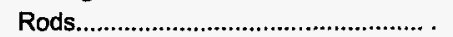 & 113.3 & 113.6 & 113.6 & 112.3 & 43,900 \\
\hline 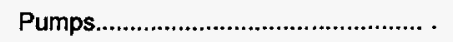 & 134.1 & 154.9 & 174.4 & 185.4 & 15,200 \\
\hline Pumping Equipment............................ & 158.4 & 177.6 & 188.9 & 192.4 & 431,400 \\
\hline Subtotal or Index ${ }^{* \star} \ldots \ldots . . . . . . . . . . . . . . . . . . .$. & 142.3 & 164.6 & 173.0 & 179.0 & 615,800 \\
\hline \multicolumn{6}{|l|}{ Gathering System: } \\
\hline 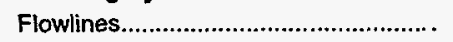 & 238.8 & 242.0 & 246.8 & 253.7 & 47,700 \\
\hline 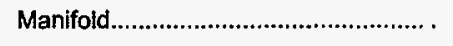 & 263.6 & 266.7 & 273.5 & 290.2 & 38,300 \\
\hline Subtotal or Index ${ }^{* *} \ldots . . . . . . . . . . . . . . . . . . .$. & 249.1 & 252.2 & 257.8 & 268.8 & 86,000 \\
\hline \multicolumn{6}{|l|}{ Lease Equipment: } \\
\hline Producing Separator........................... & 173.4 & 173.4 & 182.8 & 187.5 & 12,000 \\
\hline 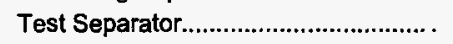 & 189.1 & 188.1 & 187.1 & 198.0 & 20,000 \\
\hline Heater Treater........................................ . & 136.8 & 145.8 & 150.3 & 154.8 & 24,000 \\
\hline Storage Tanks....................................... . & 193.2 & 198.2 & 203.0 & 207.4 & 69,700 \\
\hline Accessory Equipment.......................... & 212.9 & 220.4 & 227.2 & 227.9 & 33,500 \\
\hline Disposal System & 189.5 & 193.0 & 195.8 & 203.3 & 87,000 \\
\hline 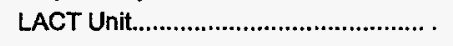 & 174.2 & 171.0 & 171.0 & 171.0 & 15,900 \\
\hline Subtotal or index ${ }^{* *}$ & 185.0 & 189.0 & 192.7 & 198.0 & 262,100 \\
\hline Total or Index & 160.1 & 176.4 & 183.5 & 189.6 & 963,900 \\
\hline
\end{tabular}


Table A3. Lease Equipment Costs and Indices for Primary Oil Production in West Texas (10 Wells Producing from 8,000 Feet by Rod Lift)

\begin{tabular}{|c|c|c|c|c|c|}
\hline \multirow{2}{*}{ Component } & \multicolumn{4}{|c|}{ Index (1976=100) } & \multirow{2}{*}{$\begin{array}{c}1997^{\star} \\
\text { Cost } \\
\text { (dollars) }\end{array}$} \\
\hline & 1994 & 1995 & 1996 & 1997 & \\
\hline \multicolumn{6}{|l|}{ Producing Equipment: } \\
\hline Tubing & 111.5 & 147.6 & 150.6 & 167.1 & 313,000 \\
\hline 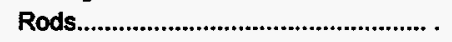 & 100.1 & 100.1 & 100.2 & 99.6 & 99,500 \\
\hline 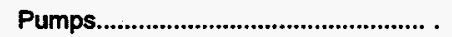 & 157.8 & 172.2 & 187.8 & 197.8 & 17,800 \\
\hline Pumping Equipment........................... . & 173.0 & 190.3 & 203.1 & 211.1 & 897,000 \\
\hline Subtotal or Index & 146.7 & 166.5 & 175.0 & 184.1 & $1,327,300$ \\
\hline \multicolumn{6}{|l|}{ Gathering System: } \\
\hline 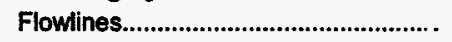 & 226.6 & 229.7 & 234.0 & 240.5 & 62,300 \\
\hline 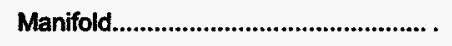 & 263.6 & 266.7 & 273.5 & 290.2 & 38,300 \\
\hline Subtotal or Index & 239.1 & 242.2 & 247.3 & 257.3 & 100,600 \\
\hline \multicolumn{6}{|l|}{ Lease Equipment: } \\
\hline Producing Separator.............................. & 173.4 & 173.4 & 182.8 & 187.5 & 42,000 \\
\hline Test Separator....................................... & 189.1 & 188.1 & 187.1 & 198.0 & 20,000 \\
\hline Heater Treater........................................ . & 136.8 & 145.8 & 150.3 & 154.8 & 24,000 \\
\hline Storage Tanks..................................... . & 193.2 & 198.2 & 203.0 & 207.4 & 69,700 \\
\hline Accessory Equipment........................... & 212.9 & 220.4 & 227.2 & 227.9 & 33,500 \\
\hline 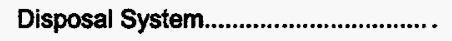 & 203.5 & 207.5 & 209.7 & 214.7 & 80,100 \\
\hline 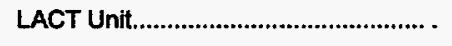 & 174.2 & 171.0 & 171.0 & 171.0 & 15,900 \\
\hline Subtotal or Index ${ }^{* *}$ & 188.9 & 193.1 & 196.7 & 201.1 & 255,200 \\
\hline Total or Index & 156.8 & 173.6 & 181.3 & 189.7 & $1,683,100$ \\
\hline \multirow[b]{2}{*}{ Component } & \multicolumn{4}{|c|}{ Index $(1976=100)$} & $1997^{*}$ \\
\hline & 1994 & 1995 & 1996 & 1997 & $\begin{array}{c}\text { Cost } \\
\text { (dollars) }\end{array}$ \\
\hline \multicolumn{6}{|l|}{ Producing Equipment: } \\
\hline 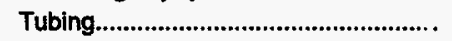 & 136.6 & 144.1 & 149.2 & 163.1 & 832,900 \\
\hline 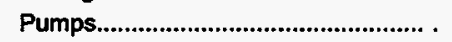 & 250.5 & 261.7 & 280.0 & 280.0 & 209,700 \\
\hline Pumping Equipment.............................. . & 171.7 & 176.2 & 182.7 & 194.2 & 345,300 \\
\hline 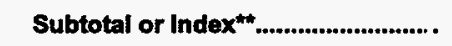 & 155.9 & 163.1 & 169.8 & 181.8 & $1,387,900$ \\
\hline \multicolumn{6}{|l|}{ Gathering System: } \\
\hline 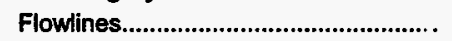 & 190.3 & 192.9 & 196.7 & 197.8 & 179,200 \\
\hline 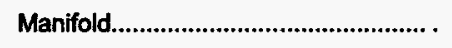 & 263.6 & 266.7 & 273.5 & 290.2 & 38,300 \\
\hline Subtotal or Index ${ }^{* *}$ & 199.6 & 202.3 & 206.5 & 209.5 & 217,500 \\
\hline \multicolumn{6}{|l|}{ Lease Equipment: } \\
\hline Producing Separator............................. & 173.4 & 173.4 & 182.8 & 187.5 & 12,000 \\
\hline Test Separator....................................... & 189.1 & 188.1 & 187.1 & 198.0 & 20,000 \\
\hline Heater Treater.................................... . & 136.8 & 145.8 & 150.3 & 154.8 & 24,000 \\
\hline Storage Tanks..................................... . & 193.2 & 198.2 & 203.0 & 207.4 & 69,700 \\
\hline Accessory Equipment........................... & 212.9 & 220.4 & 227.2 & 227.9 & 33,500 \\
\hline 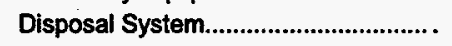 & 203.5 & 207.5 & 209.7 & 214.7 & 80,100 \\
\hline 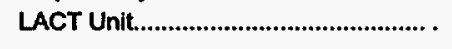 & 174.2 & 171.0 & 171.0 & 171.0 & 15,900 \\
\hline Subtotal or Index & 188.9 & 193.1 & 196.7 & 201.1 & 255,200 \\
\hline Total or Index ${ }^{\star *} \ldots . . . . .$. & 164.7 & 171.1 & 177.1 & 187.2 & $1,860,600$ \\
\hline
\end{tabular}


Table A5. Direct Annual Operating Costs and Indices for Primary Oil Production in West Texas (10 Wells Producing from 2,000 Feet by Rod Lift)

\begin{tabular}{|c|c|c|c|c|c|}
\hline \multirow{2}{*}{ Component } & \multicolumn{4}{|c|}{ Index $(1976=100)$} & \multirow{2}{*}{$\begin{array}{c}1997^{\star} \\
\text { Cost } \\
\text { (dolllars) }\end{array}$} \\
\hline & 1994 & 1995 & 1996 & 1997 & \\
\hline \multicolumn{6}{|l|}{ Normal Daily Expense: } \\
\hline Supervision and Overhead.................................... . & 316.7 & 336.7 & 340.0 & 355.0 & 21,300 \\
\hline Labor (pumper) & 255.4 & 255.4 & 255.4 & 255.4 & $13 ; 900$ \\
\hline 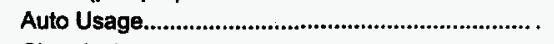 & 261.5 & 273.1 & 284.6 & 296.2 & 7,700 \\
\hline 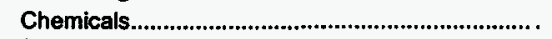 & 203.7 & 203.7 & 203.7 & 214.8 & 5,800 \\
\hline 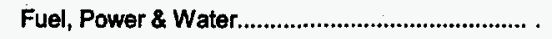 & 266.7 & 237.5 & 316.7 & 330.6 & 23,800 \\
\hline Operative Supplies................................................ . & 233.3 & 233.3 & 233.3 & 233.3 & 1,400 \\
\hline 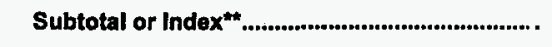 & 267.2 & 264.9 & 288.3 & 297.7 & $7: 3,900$ \\
\hline \multicolumn{6}{|l|}{ Surface Maintenance, Repair \& Services: } \\
\hline 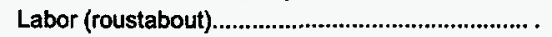 & 231.0 & 231.0 & 231.0 & 231.0 & 6,700 \\
\hline Supplies \& Services................................................. & 234.4 & 234.4 & 234.4 & 234.4 & 7,500 \\
\hline 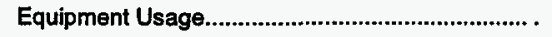 & 253.8 & 253.8 & 253.8 & 253.8 & 3,300 \\
\hline 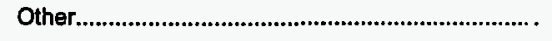 & 173.3 & 173.3 & 173.3 & 173.3 & 2,600 \\
\hline Subtotal or Index & 225.8 & 225.8 & 225.8 & 225.8 & 20,100 \\
\hline \multicolumn{6}{|l|}{ Subsurface Maintenance, Repair \& Services: } \\
\hline Workover Rig Services.............................................. . & 197.4 & 197.4 & 189.7 & 197.4 & 7,700 \\
\hline 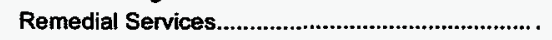 & 137.5 & 137.5 & 137.5 & 143.8 & 2,300 \\
\hline 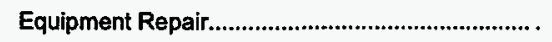 & 144.4 & 151.1 & 155.6 & 164.4 & 7,400 \\
\hline 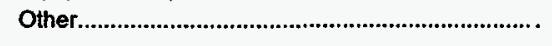 & 200.0 & 200.0 & 200.0 & 200.0 & 400 \\
\hline Subtotal or Index & 164.7 & 167.6 & 166.7 & 174.5 & 17,800 \\
\hline Total or Index & 236.2 & 235.5 & 248.9 & 256.1 & 116,800 \\
\hline
\end{tabular}

Table A6. Direct Annual Operating Costs and Indices for Primary Oil Production in West Texas (10 Wells Producing from 4,000 Feet by Rod Lift)

\begin{tabular}{|c|c|c|c|c|c|}
\hline \multirow{2}{*}{ Component } & \multicolumn{4}{|c|}{ Index $(1976=100)$} & \multirow{2}{*}{$\begin{array}{c}1997^{\star} \\
\text { Cost } \\
\text { (dollars) }\end{array}$} \\
\hline & 1994 & 1995 & 1996 & 1997 & \\
\hline \multicolumn{6}{|l|}{ Normal Daily Expense: } \\
\hline 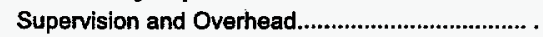 & 316.2 & 335.3 & 339.7 & 355.9 & 24,200 \\
\hline Labor (pumper) & 255.4 & 255.4 & 255.4 & 255.4 & 18,900 \\
\hline 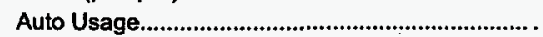 & 261.5 & 273.1 & 284.6 & 296.2 & 7,700 \\
\hline 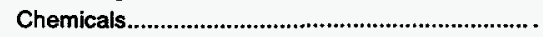 & 218.5 & 218.5 & 214.8 & 229.6 & 6,200 \\
\hline 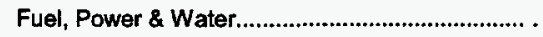 & 265.9 & 236.4 & 318.2 & 334.1 & 29,400 \\
\hline 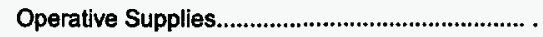 & 250.0 & 250.0 & 250.0 & 250.0 & 1,500 \\
\hline Subtotal or Index & 269.9 & 266.4 & 293.1 & 304.2 & 87,900 \\
\hline \multicolumn{6}{|l|}{ Surface Maintenance, Repair \& Services: } \\
\hline Labor (roustabout) & 231.0 & 231.0 & 231.0 & 231.0 & 6,700 \\
\hline 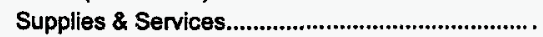 & 231.0 & 231.0 & 231.0 & 231.0 & 6,700 \\
\hline 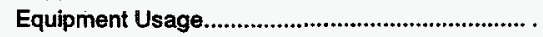 & 261.5 & 261.5 & 261.5 & 261.5 & 3,400 \\
\hline 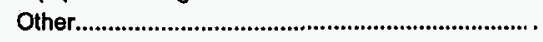 & 183.3 & 183.3 & 183.3 & 183.3 & 4,400 \\
\hline Subtotal or Index & 225.0 & 225.0 & 226.0 & 226.0 & 22,600 \\
\hline \multicolumn{6}{|l|}{ Subsurtace Maintenance, Repair \& Services: } \\
\hline Workover Rig Services............................................ & 195.5 & 195.5 & 189.4 & 195.5 & 12,900 \\
\hline 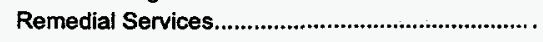 & 156.5 & 160.9 & 160.9 & 165.2 & 3,800 \\
\hline 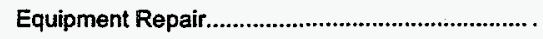 & 128.6 & 149.0 & 161.2 & 171.4 & 8,400 \\
\hline Other & 166.7 & 166.7 & 166.7 & 166.7 & 500 \\
\hline Subtotal or Index & 165.2 & 173.0 & 174.5 & 181.6 & 25,600 \\
\hline Total or Index & 233.6 & 233.8 & 248.9 & 256.8 & 136,100 \\
\hline
\end{tabular}


Table A7. Direct Annual Operating Costs and Indices for Primary Oil Production in West Texas (10 Wells Producing from 8,000 Feet by Rod Lift)

\begin{tabular}{|c|c|c|c|c|c|}
\hline \multirow{2}{*}{ Component } & \multicolumn{4}{|c|}{ Index $(1976=100)$} & \multirow{2}{*}{$\begin{array}{c}1997^{\star} \\
\text { Cost } \\
\text { (dollars) }\end{array}$} \\
\hline & 1994 & 1995 & 1996 & 1997 & \\
\hline \multicolumn{6}{|l|}{ Normal Daily Expense: } \\
\hline 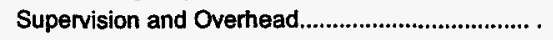 & 319.0 & 339.2 & 343.0 & 359.5 & 28,400 \\
\hline Labor (pumper) & 255.4 & 255.4 & 255.4 & 255.4 & 18,900 \\
\hline 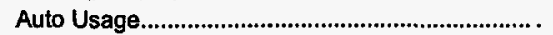 & 261.5 & 273.1 & 284.6 & 296.2 & 7,700 \\
\hline 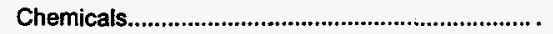 & 200.0 & 196.6 & 196.6 & 210.3 & 6,100 \\
\hline 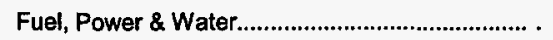 & 264.1 & 232.8 & 318.8 & 335.2 & 42,900 \\
\hline 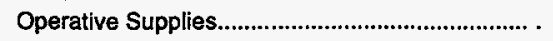 & 228.6 & 228.6 & 228.6 & 228.6 & 1,600 \\
\hline Subtotal or Index & 268.5 & 262.1 & 295.9 & 307.9 & 105,600 \\
\hline \multicolumn{6}{|l|}{ Surface Maintenance, Repair \& Services: } \\
\hline 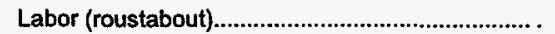 & 231.0 & 231.0 & 231.0 & 231.0 & 6,700 \\
\hline 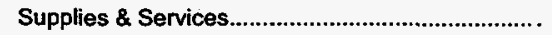 & 230.8 & 230.8 & 230.8 & 233.3 & 9,100 \\
\hline 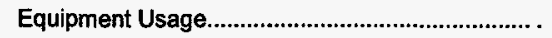 & 220.0 & 220.0 & 220.0 & 220.0 & 3,300 \\
\hline 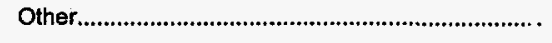 & 176.7 & 176.7 & 176.7 & 176.7 & 5,300 \\
\hline Subtotal or Index" & 215.0 & 215.0 & 215.0 & 215.9 & 24,400 \\
\hline \multicolumn{6}{|l|}{ Subsurface Maintenance, Repair \& Services: } \\
\hline 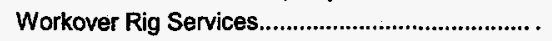 & 218.1 & 218.1 & 210.4 & 218.1 & 31,400 \\
\hline 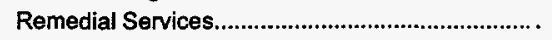 & 180.0 & 182.9 & 181.4 & 188.6 & 13,200 \\
\hline 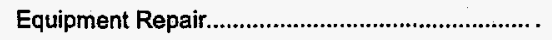 & 163.3 & 183.3 & 191.7 & 201.7 & 12,100 \\
\hline 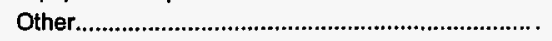 & 166.7 & 177.8 & 177.8 & 177.8 & 1,600 \\
\hline Subtotal or Index & 195.4 & 200.7 & 198.2 & 206.0 & 58,300 \\
\hline Total or Index ${ }^{* *} \ldots \ldots$ & 232.3 & 231.4 & 246.1 & 254.8 & 188,300 \\
\hline
\end{tabular}

Table A8. Direct Annual Operating Costs and Indices for Primary Oil Production in West Texas (10 Wells Producing from 12,000 Feet by Hydraulic Lift)

\begin{tabular}{|c|c|c|c|c|c|}
\hline \multirow{2}{*}{ Component } & \multicolumn{4}{|c|}{ Index $(1976=100)$} & \multirow{2}{*}{$\begin{array}{c}1997^{*} \\
\text { Cost } \\
\text { (dollars) }\end{array}$} \\
\hline & 1994 & 1995 & 1996 & 1997 & \\
\hline \multicolumn{6}{|l|}{ Normal Daily Expense: } \\
\hline 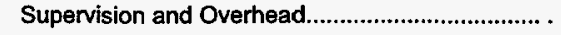 & 320.4 & 341.8 & 344.9 & 362.2 & 35,500 \\
\hline 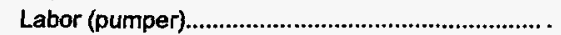 & 255.4 & 255.4 & 255.4 & 255.4 & 18,900 \\
\hline Auto Usage & 261.5 & 273.1 & 284.6 & 296.2 & 7,700 \\
\hline 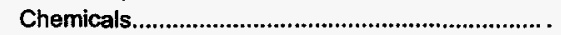 & 190.9 & 190.9 & 190.9 & 203.0 & 6,700 \\
\hline 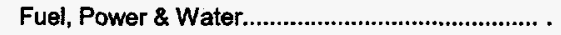 & 261.4 & 230.1 & 318.2 & 334.1 & 58,800 \\
\hline Operative Supplies....................................................... . & 280.0 & 280.0 & 280.0 & 290.0 & 2,900 \\
\hline Subtotal or Index & 269.1 & 261.6 & 300.2 & 312.9 & 130,500 \\
\hline \multicolumn{6}{|l|}{ Surface Maintenance, Repair \& Services: } \\
\hline Labor (roustabout) & 231.0 & 231.0 & 231.0 & 231.0 & 6,700 \\
\hline 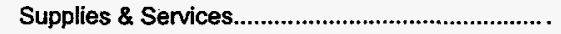 & 212.9 & 212.9 & 214.9 & 220.8 & 22,300 \\
\hline 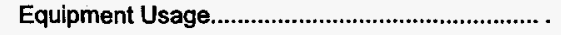 & 220.0 & 220.0 & 220.0 & 220.0 & 3,300 \\
\hline 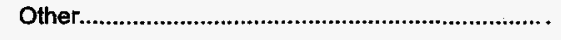 & 216.7 & 216.7 & 216.7 & 233.3 & 1,400 \\
\hline Subtotal or Index & 217.2 & 217.2 & 218.5 & 223.2 & 33,700 \\
\hline \multicolumn{6}{|l|}{ Subsurface Maintenance, Repair \& Services: } \\
\hline 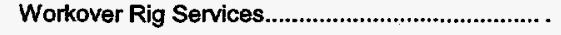 & 192.4 & 192.4 & 189.4 & 192.4 & 12,700 \\
\hline Remedial Services. & 187.4 & 189.1 & 188.2 & 195.8 & 23,300 \\
\hline 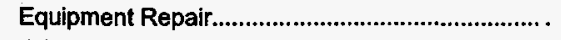 & 403.9 & 421.8 & 450.8 & 451.4 & 80,800 \\
\hline 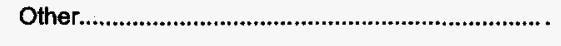 & 175.0 & 175.0 & 175.0 & 183.3 & 2,200 \\
\hline Subtotal or Index & 291.0 & 300.0 & 313.0 & 316.5 & 119,000 \\
\hline 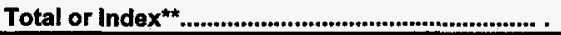 & 269.5 & 269.8 & 292.3 & 300.0 & 283,200 \\
\hline
\end{tabular}


Table A9. Additional Lease Equipment Costs and Indices for Secondary Oll Production in West Texas (10 Wells Producing from 2,000 Feet by Rod Lift and 11 Water Injection Wells)

\begin{tabular}{|c|c|c|c|c|c|}
\hline \multirow{2}{*}{ Component } & \multicolumn{4}{|c|}{ Index $(1976=100)$} & \multirow{2}{*}{$\begin{array}{c}1997^{\star} \\
\text { Cost } \\
\text { (dollars) }\end{array}$} \\
\hline & 1994 & 1995 & 1996 & 1997 & \\
\hline \multicolumn{6}{|l|}{ Injection Equipment: } \\
\hline 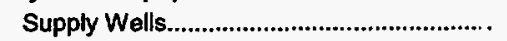 & 175.0 & 203.4 & 210.9 & 228.2 & 166,800 \\
\hline 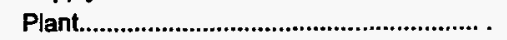 & 290.2 & 299.7 & 311.0 & 321.4 & 108,300 \\
\hline 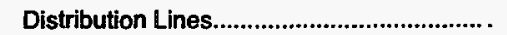 & 180.6 & 178.4 & 178.7 & 182.7 & 77,100 \\
\hline 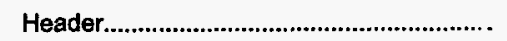 & 258.9 & 265.2 & 272.5 & 284.5 & 58,900 \\
\hline 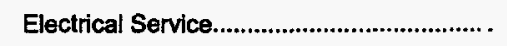 & 327.2 & 330.6 & 334.3 & 339.2 & 89,900 \\
\hline Subtotal or Index & 225.4 & 238.3 & 244.3 & 255.4 & 501,000 \\
\hline \multicolumn{6}{|l|}{ Producing Equipment: } \\
\hline Tubing Replacement................................... . & 120.4 & 143.1 & 145.7 & 157.7 & 61,800 \\
\hline 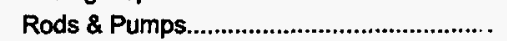 & 119.8 & 121.1 & 122.6 & 123.6 & 39,300 \\
\hline 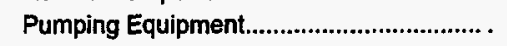 & 281.6 & 335.7 & 335.7 & 545.9 & 53,500 \\
\hline Subtotal or Index ${ }^{* *} \ldots$ & 139.7 & 157.8 & 159.7 & 191.3 & 154,600 \\
\hline \multicolumn{6}{|l|}{ injection Wells: } \\
\hline Subtotal or Index ${ }^{* *}$ & 138.3 & 157.0 & 235.5 & 270.8 & $1,434,900$ \\
\hline Total or Index ${ }^{* *} \ldots \ldots \ldots$ & 159.6 & 176.8 & 230.1 & 259.1 & $2,090,500$ \\
\hline
\end{tabular}

Table A10. Additional Lease Equipment Costs and Indices for Secondary Oil Production in West Texas (10 Wells Producing from 4,000 Feet by Rod Lift and 11 Water Injection Wells)

\begin{tabular}{|c|c|c|c|c|c|}
\hline \multirow{2}{*}{ Component } & \multicolumn{4}{|c|}{ Index $(1976=100)$} & \multirow{2}{*}{$\begin{array}{c}1997^{\star *} \\
\text { Cost } \\
\text { (dollars) }\end{array}$} \\
\hline & 1994 & 1995 & 1996 & 1997 & \\
\hline \multicolumn{6}{|l|}{ Injection Equipment: } \\
\hline 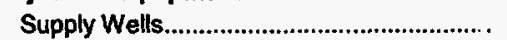 & 175.0 & 203.3 & 210.9 & 228.2 & 166,800 \\
\hline Plant & 286.4 & 296.2 & 307.1 & 317.8 & 107,400 \\
\hline 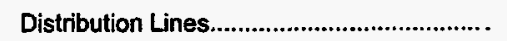 & 180.6 & 178.4 & 178.7 & 182.7 & 77,100 \\
\hline 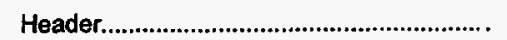 & 258.9 & 265.2 & 272.5 & 284.5 & 58,900 \\
\hline 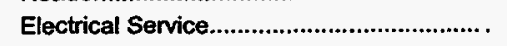 & 238.0 & 240.5 & 243.2 & 246.7 & 90,800 \\
\hline Subtotal or Index & 214.0 & 226.2 & 232.0 & 242.5 & 501,000 \\
\hline \multicolumn{6}{|l|}{ Producing Equipment: } \\
\hline 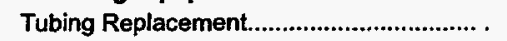 & 117.2 & 158.7 & 161.3 & 176.3 & 134,500 \\
\hline 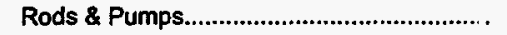 & 112.9 & 113.9 & 114.6 & 115.2 & 60,600 \\
\hline 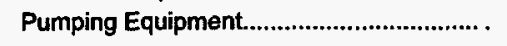 & 141.4 & 162.5 & 175.8 & 199.6 & 400,500 \\
\hline Subtotal or Index ${ }^{* *}$ & 131.2 & 153.9 & 162.7 & 180.7 & 595,600 \\
\hline \multicolumn{6}{|l|}{ Injection Wells:"tik } \\
\hline Subtotal or Index ${ }^{* *}$ & 152.8 & 173.7 & 182.4 & 228.0 & $2,589,800$ \\
\hline Total or Index & 156.1 & 176.3 & 184.7 & 220.5 & $3,685,400$ \\
\hline
\end{tabular}


Table A11. Additional Lease Equipment Costs and Indices for Secondary Oil Production in West Texas (10 Wells Producing from 8,000 Feet by Rod Lift and 11 Water Injection Wells)

\begin{tabular}{|c|c|c|c|c|c|}
\hline \multirow{2}{*}{ Component } & \multicolumn{4}{|c|}{ Index $(1976=100)$} & \multirow{2}{*}{$\begin{array}{c}\text { 1997* } \\
\text { Cost } \\
\text { (dollars) } \\
\end{array}$} \\
\hline & 1994 & 1995 & 1996 & 1997 & \\
\hline \multicolumn{6}{|l|}{ Injection Equipment: } \\
\hline 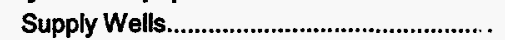 & 174.9 & 206.3 & 214.3 & 233.2 & 302,700 \\
\hline 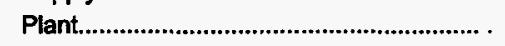 & 302.4 & 312.4 & 322.6 & 333.8 & 220,300 \\
\hline 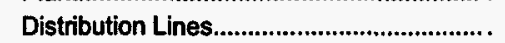 & 181.1 & 178.9 & 178.9 & 183.1 & 116,100 \\
\hline 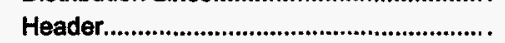 & 237.5 & 243.1 & 249.4 & 258.4 & 69,000 \\
\hline Electrical Service & 252.5 & 253.5 & 255.0 & 259.6 & 138,900 \\
\hline Subtotal or Index $x^{* *}$ & 218.0 & 232.1 & 237.9 & 249.6 & 847,000 \\
\hline \multicolumn{6}{|l|}{ Producing Equipment: } \\
\hline 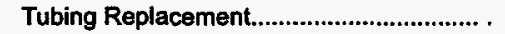 & 116.1 & 151.1 & 154.0 & 169.3 & 326,800 \\
\hline 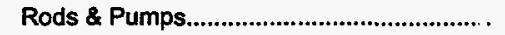 & 102.3 & 101.5 & 100.9 & 101.4 & 120,100 \\
\hline 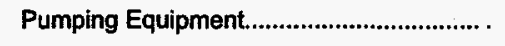 & 143.1 & 161.6 & 175.8 & 196.1 & 737,800 \\
\hline Subtotal or Index & 128.5 & 148.3 & 156.8 & 172.3 & $1,184,700$ \\
\hline \multicolumn{6}{|l|}{ Injection Wells:" } \\
\hline Subtotal or Index $x^{* *}$ & 118.3 & 135.1 & 141.9 & 177.3 & $5,285,300$ \\
\hline Total or Index ${ }^{*} \ldots$ & 128.5 & 145.6 & 152.6 & 182.6 & $7,317,000$ \\
\hline
\end{tabular}

Table A12. Direct Annual Operating Costs and Indices for Secondary Oll Production in West Texas (10 Welis Producing from 2,000 Feet by Rod Lift and 11 Water Injection Wells)

\begin{tabular}{|c|c|c|c|c|c|}
\hline \multirow{2}{*}{ Component } & \multicolumn{4}{|c|}{ Index $(1976=100)$} & \multirow{2}{*}{$\begin{array}{c}\text { 1997* } \\
\text { Cost } \\
\text { (dollars) }\end{array}$} \\
\hline & 1994 & 1995 & 1996 & 1997 & \\
\hline \multicolumn{6}{|l|}{ Normal Daily Expense: } \\
\hline 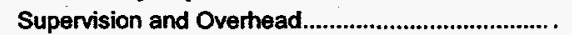 & 320.0 & 339.3 & 343.4 & 357.9 & 51,900 \\
\hline 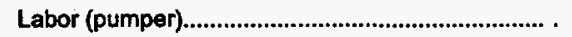 & 295.0 & 295.0 & 295.0 & 295.0 & 35,400 \\
\hline 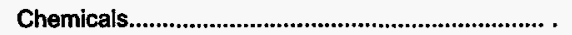 & 202.9 & 200.0 & 200.0 & 211.4 & 7,400 \\
\hline 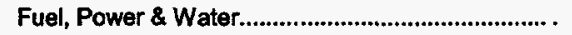 & 259.9 & 256.3 & 265.6 & 267.2 & 80,700 \\
\hline 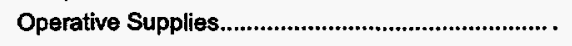 & 266.7 & 260.0 & 266.7 & 266.7 & 4,000 \\
\hline Subtotal or Index $x^{* *}$ & 277.8 & 280.2 & 285.9 & 290.8 & 179,400 \\
\hline \multicolumn{6}{|l|}{ Surface Maintenance, Repair \& Services: } \\
\hline 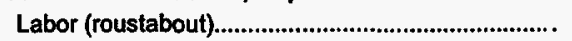 & 231.9 & 231.9 & 231.9 & 231.9 & 16,000 \\
\hline 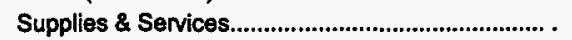 & 216.4 & 210.9 & 215.5 & 220.0 & 24,200 \\
\hline 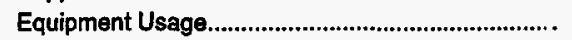 & 282.6 & 282.6 & 282.6 & 282.6 & 6,500 \\
\hline 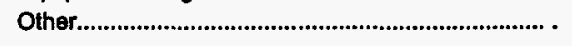 & 186.7 & 186.7 & 186.7 & 186.7 & 2,800 \\
\hline Subtotal or Index & 226.3 & 223.5 & 225.8 & 228.1 & 49,500 \\
\hline \multicolumn{6}{|l|}{ Subsurface Maintenance, Repair \& Services: } \\
\hline 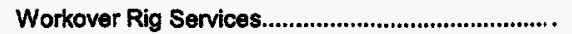 & 229.4 & 229.4 & 221.0 & 229.4 & 32,800 \\
\hline 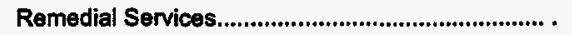 & 187.8 & 190.2 & 190.2 & 197.6 & 8,100 \\
\hline 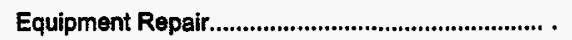 & 137.7 & 137.7 & 132.1 & 137.7 & 7,300 \\
\hline Other & 191.4 & 194.3 & 197.1 & 200.0 & 7,000 \\
\hline Subtotal or Index & 200.4 & 201.1 & 196.0 & 202.9 & 55,200 \\
\hline Total or Index $* 4$ & 248.6 & 249.6 & 252.0 & 256.9 & 284,100 \\
\hline
\end{tabular}


Table A13. Direct Annual Operating Costs and Indices for Secondary Oil Production in West Texas (10 Wells Producing from 4,000 Feet by Rod Lift and 11 Water Injection Wells)

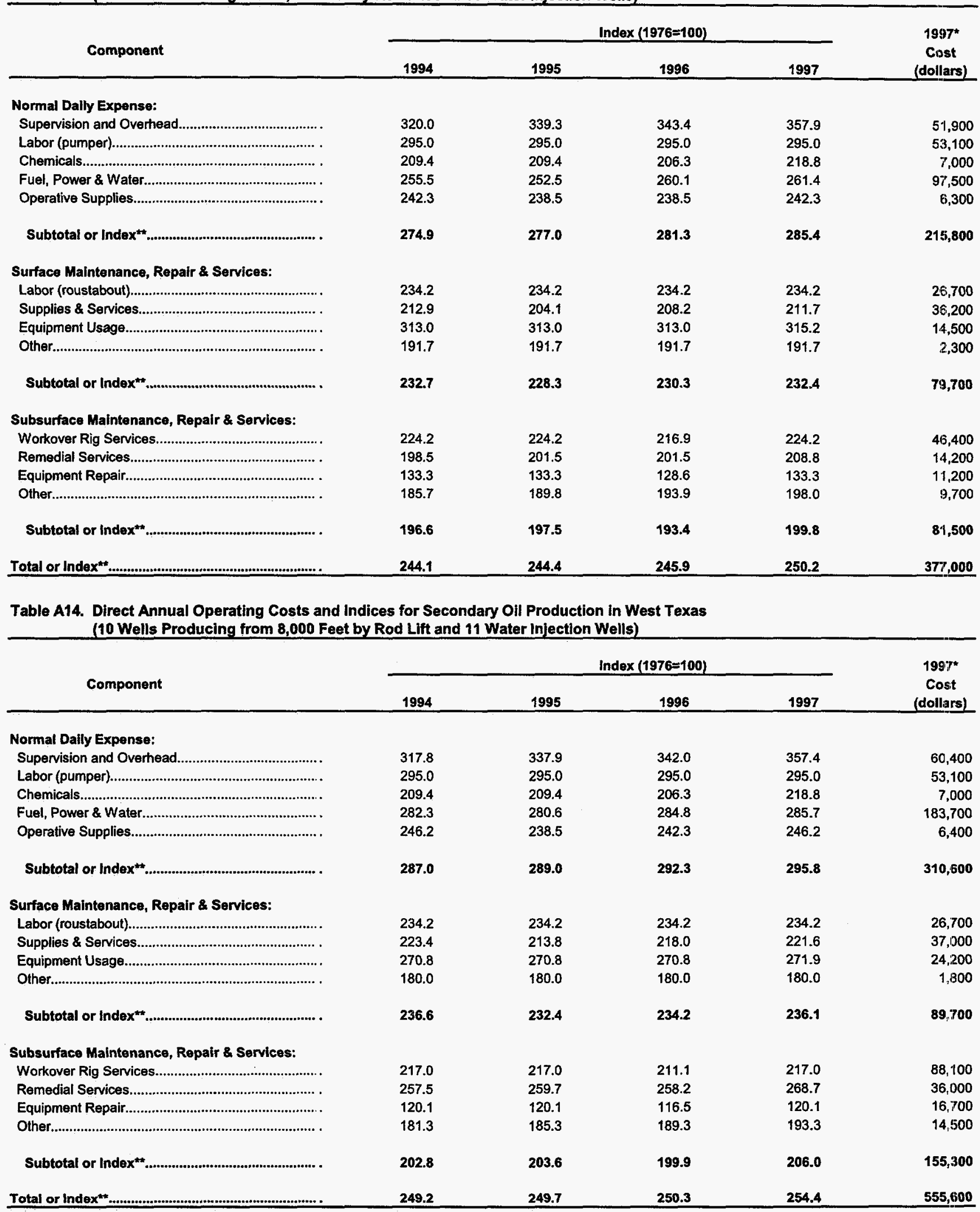


Table A15. Detailed Lease Equipment List for 2,000-Foot Wells in West Texas (10 Producing Wells)

\section{Tubing $(20,000$ feet $)$}

Size: $2-3 / 8$ inches

Weight: 4.7 pounds per foot

Grade: $\mathrm{H}-40$

\section{Sucker Rods $(20,000$ feet $)$}

Size: $5 / 8$ inches

API class: $K$

Rod Pump (10)

API type: TH

Size: 2 by $1-3 / 4$ inches by 9 feet

Pumping Unit (10)

API size: C57D-76-54

Engine: 9 horsepower single cylinder

\section{Oil Flowline $(11,500$ feet $)$}

Size: $2-3 / 8$ inches

Material: polyvinyl chloride 1120

Weight: 0.43 pounds per foot

Pressure rating: 160 pounds per square inch

\section{Manifold (1)}

Valves: 2-inch, 3-way, 2-position, electric operated (10)

\section{Production Separator (1)}

Type: vertical

Size: 30 inches by 10 feet

Capacity: 2,700 barrels of fluid per day and 5.7 million cubic feet of gas per day

Vapor Recovery Unit (1)

Capacity: $\mathbf{4 0}$ thousand cubic feet of gas per day
Test Separator (1)

Type: vertical

Size: 24 inches by $7-1 / 2$ feet

Capacity: 1.290 barrels of fluid per day

Working pressure: 125 pounds per square inch

Net oil computer: Electronic

Heater Treater (1)

Working pressure: 50 pounds per square inch

Size: 4 feet by $27-1 / 2$ feet

Oil Storage Tanks (2)

Storage capacity: 2,000 barrels

Type: 10-gauge, bolted steel

Construction: gas tight

Size: 30 feet by 16 feet

Water Disposal Pump (1)

Type: Quintuplex

Plungers: $1-1 / 2$ inches

Working pressure: 1,000 pounds per square inch

Electric motor: 20 horsepower

Water Disposal Line $(2,000$ feet $)$

Size: $2-3 / 8$ inches

Weight: 3.75 pounds per foot

Grade: $B$

Mill test: 2,500 pounds per square inch

LACT Unit (1)

Capacity: 2,000 barrels per day

Working pressure: 125 pounds per square inch

Source: Energy Information Administration, Office of Oil and Gas. 
Tubing $(40,000$ feet)

Size: $2-3 / 8$ inches

Weight: 4.7 pounds per foot

Grade: J-55

Sucker Rods $(20,000$ feet $)$

Size: $5 / 8$ inches $(24,000$ feet $)$

Size: $3 / 4$ inches $(16,000$ feet $)$

API class: $K$

Rod Pump (10)

API type: RWBC

Size: 2 by $1-1 / 2$ inches by 9 feet

Pumping Unit (10)

API size: M160D-173-74

Engine: 12 horsepower single cylinder

\section{Oil Flowline (16,000 feet)}

Size: $2-3 / 8$ inches

Material: polyvinyl chloride 1120

Weight: 0.43 pounds per foot

Pressure rating: 160 pounds per square inch

Manifold (1)

Valves: 2-inch, 3-way, 2-position, electric operated (10)

Production Separator (1)

Type: vertical

Size: 30 inches by 10 feet

Capacity: 2,700 barrels of fluid per day and 5.7 million

cubic feet of gas per day

Vapor Recovery Unit (1)

Capacity: 40 thousand cubic feet of gas per day
Test Separator (1)

Type: vertical

Size: 24 inches by 7-1/2 feet

Capacity: 1.290 barrels of fluid per day

Working pressure: 125 pounds per square inch

Net oil computer: Electronic

Heater Treater (1)

Working pressure: 50 pounds per square inch

Size: 4 feet by $27-1 / 2$ feet

Oil Storage Tanks (2)

Storage capacity: 2,000 barrels

Type: 10-gauge, bolted steel

Construction: gas tight

Size: 30 feet by 16 feet

Water Disposal Pump (1)

Type: Quintuplex

Plungers: $1-1 / 2$ inches

Working pressure: 1,000 pounds per square inch

Electric motor: 20 horsepower

Water Disposal Line (2,000 feet)

Size: $2-3 / 8$ inches

Weight: 3.75 pounds per foot

Grade: B

Mill test: 2,500 pounds per square inch

\section{LACT Unit (1)}

Capacity: 2,000 barrels per day

Working pressure: 125 pounds per square inch

Source: Energy Information Administration, Office of Oil and Gas. 
Table A17. Detailed Lease Equipment List for 8,000-Foot Wells in West Texas (10 Producing Wells)

\section{Tubing $(80,000$ feet $)$}

Size: $2-7 / 8$ inches

Weight: 6.5 pounds per foot

Grade: J-55

\section{Sucker Rods (80,000 feet)}

Size: 1 inch $(15,250$ feet $)$

Size: $7 / 8$ inches $(17,500$ feet)

Size: $3 / 4$ inches $(47,250$ feet)

API class: $K$

Rod Pump (10)

API type: RWBC

Size: $2-1 / 2$ by $1-1 / 4$ inches by 20 feet

Pumping Unit (10)

API size: M456D-305-144

Engine: 32 horsepower single cylinder

Oil Flowline $(23,200$ feet $)$

Size: $2-3 / 8$ inches

Material: polyvinyl chloride 1120

Weight: 0.43 pounds per foot

Pressure rating: 160 pounds per square inch

Manifold (1)

Valves: 2-inch, 3-way, 2-position, electric operated (10)

Production Separator (1)

Type: vertical

Size: 30 inches by 10 feet

Capacity: 2,700 barrels of fluid per day and 5.7 million

cubic feet of gas per day

\section{Vapor Recovery Unit (1)}

Capacity: 40 thousand cubic feet of gas per day
Test Separator (1)

Type: vertical

Size: 24 inches by $7-1 / 2$ feet

Capacity: 1.290 barrels of fluid per day

Working pressure: 125 pounds per square inch

Net oil computer: Electronic

Heater Treater (1)

Working pressure: 50 pounds per square inch Size: 4 feet by $27-1 / 2$ feet

Oil Storage Tanks (2)

Storage capacity: 2,000 barrels

Type: 10-gauge, bolted steel

Construction: gas tight

Size: 30 feet by 16 feet

Water Disposal Pump (1)

Type: Quintuplex

Plungers: $1-1 / 2$ inches

Working pressure: 1,000 pounds per square inch

Electric motor: 20 horsepower

Water Disposal Line $(3,400$ feet $)$

Size: $2-3 / 8$ inches

Weight: 3.75 pounds per foot

Grade: B

Mill test: 2,500 pounds per square inch

LACT Unit (1)

Capacity: 2,000 barrels per day

Working pressure: 125 pounds per square inch

Source: Energy Information Administration, Office of Oil and Gas. 
Tubing $(240,000$ feet $)$

Size: $2-7 / 8$ inches $(120,000$ feet $)$

Weight: 6.5 pounds per foot

Grade: N-80

Size: 1.66 inches $(120,000$ feet $)$

Weight: 2.4 pounds per foot

Grade: J-55

\section{Hydraulic Bottom Hole Pump (10)}

Size: 2 by $1-3 / 8$ by $1-3 / 16$ inches

Surface Pumping Equipment (4)

Type: Triplex

Engine: 6-cylinder, 100 horsepower

Power Oil Flowlines $(23,200$ feet $)$

Size: $1-1 / 2$ inches

Grade: J-55

Power Oil Tank (1)

Storage capacity: 750 barrels

Type: 10-gauge, bolted steel

Construction: gas tight

Size: $15-1 / 2$ feet by 24 feet

Oil Flowlines $(23,200$ feet)

Size: 2-3/8 inches

Material: polyvinyl chloride 1120

Weight: 0.43 pounds per foot

Pressure rating: 160 pounds per square inch

\section{Manifold (1)}

Valves: 2-inch, 3-way, 2-position, electric operated (10)

\section{Production Separator (1)}

Type: vertical

Size: 30 inches by 10 feet

Capacity: 2,700 barrels of fluid per day and 5.7 million

cubic feet of gas per day
Test Separator (1)

Type: vertical

Size: 24 inches by $7-1 / 2$ feet

Capacity: 1.290 barrels of fluid per day

Working pressure: 125 pounds per square inch

Net oil computer: Electronic

Heater Treater (1)

Working pressure: 50 pounds per square inch Size: 4 feet by $27-1 / 2$ feet

Oil Storage Tanks (2)

Storage capacity: 2,000 barrels

Type: 10-gauge, bolted steel

Construction: gas tight

Size: 30 feet by 16 feet

Water Disposal Pump (1)

Type: Quintuplex

Plungers: $1-1 / 2$ inches

Working pressure: 1,000 pounds per square inch

Electric motor: 20 horsepower

Water Disposal Line (2,000 feet)

Size: 2-3/8 inches

Weight: 3.75 pounds per foot

Grade: B

Mill test: 2,500 pounds per square inch

\section{LACT Unit (1)}

Capacity: 2,000 barrels per day

Working pressure: 125 pounds per square inch

Vapor Recovery Unit (1)

Capacity: 40 thousand cubic feet of gas per day

Source: Energy Information Administration, Office of Oil and Gas. 
Table B1. Lease Equipment Costs and Indices for Primary Oll Production in South Texas (10 Wells Producing from 2,000 Feet by Rod Lift)

\begin{tabular}{|c|c|c|c|c|c|}
\hline \multirow{2}{*}{ Component } & \multicolumn{4}{|c|}{ Index $(1976=100)$} & \multirow{2}{*}{$\begin{array}{c}1997^{*} \\
\text { Cost } \\
\text { (dollars) }\end{array}$} \\
\hline & 1994 & 1995 & 1996 & 1997 & \\
\hline \multicolumn{6}{|l|}{ Producing Equipment: } \\
\hline Tubing & 111.2 & 155.5 & 158.5 & 175.6 & 62,700 \\
\hline 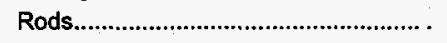 & 118.9 & 119.4 & 119.4 & 124.9 & 25,100 \\
\hline 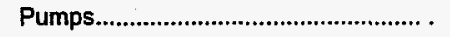 & 147.6 & 152.4 & 157.1 & 166.7 & 14,000 \\
\hline Pumping Equipment.............................. & 177.3 & 189.5 & 201.0 & 234.6 & 306,100 \\
\hline Subtotal or Index ${ }^{* k} \ldots . . . . . . . . . . . . . . . . . . . . . . .$. & 157.9 & 174.4 & 182.9 & 209.5 & 407,900 \\
\hline \multicolumn{6}{|l|}{ Gathering System: } \\
\hline 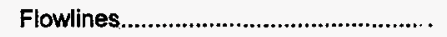 & 308.1 & 311,4 & 316.8 & 322.1 & 48,000 \\
\hline 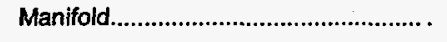 & 263.4 & 266.4 & 273.1 & 289.6 & 38,800 \\
\hline Subtotal or Index & 286.9 & 290.1 & 296.1 & 306.7 & 86,800 \\
\hline \multicolumn{6}{|l|}{ Lease Equipment: } \\
\hline 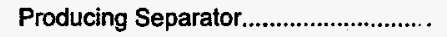 & 173.4 & 173.4 & 182.8 & 187.5 & 12,000 \\
\hline 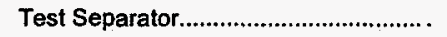 & 189.1 & 188.1 & 187.1 & 198.0 & 20,000 \\
\hline Heater Treater....................................... . & 136.8 & 145.8 & 150.3 & 154.8 & 24,000 \\
\hline 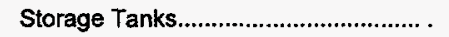 & 198.8 & 203.9 & 208.7 & 211.9 & 71,000 \\
\hline Accessory Equipment........................... & 216.3 & 223.8 & 229.9 & 229.9 & 33,800 \\
\hline 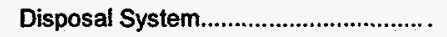 & 192.3 & 195.8 & 198.6 & 206.5 & 88,600 \\
\hline 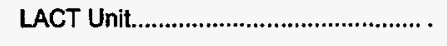 & 174.2 & 171.0 & 171.0 & 171.0 & 15,900 \\
\hline 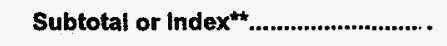 & 187.7 & 191.7 & 195.4 & 200.4 & 265,300 \\
\hline Total or Index & 179.3 & 190.1 & 196.6 & 213.8 & 760,000 \\
\hline
\end{tabular}

Table B2. Lease Equipment Costs and Indices for Primary Oil Production in South Texas (10 Wells Producing from 4,000 Feet by Gas Lift)

\begin{tabular}{|c|c|c|c|c|c|}
\hline \multirow{2}{*}{ Component } & \multicolumn{4}{|c|}{ Index $(1976=100)$} & \multirow{2}{*}{$\begin{array}{c}\text { 1997* } \\
\text { Cost } \\
\text { (dollars) }\end{array}$} \\
\hline & 1994 & 1995 & 1996 & 1997 & \\
\hline \multicolumn{6}{|l|}{ Producing Equipment: } \\
\hline Tubing & 107.5 & 151.1 & 154.0 & 170.9 & 123,700 \\
\hline Valves and Mandrels.............................. & 390.1 & 394.5 & 399.2 & 568.4 & 143,800 \\
\hline Pumping Equipment................................. . & 163.9 & 169.9 & 176.0 & 212.0 & 221,300 \\
\hline Subtotal or Index ${ }^{\star \star}$ & 172.0 & 191.3 & 196.0 & 241.9 & 488,800 \\
\hline \multicolumn{6}{|l|}{ Gathering System: } \\
\hline 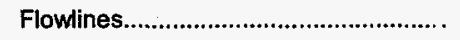 & 184.1 & 186.0 & 201.7 & 209.6 & 180,900 \\
\hline 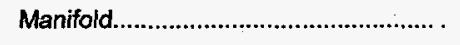 & 263.4 & 266.4 & 273.1 & 289.6 & 38,800 \\
\hline 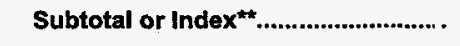 & 194.8 & 196.8 & 211.3 & 220.4 & 219,700 \\
\hline \multicolumn{6}{|l|}{ Lease Equipment: } \\
\hline Producing Separator.................................. & 173.4 & 173.4 & 182.8 & 187.5 & 12,000 \\
\hline 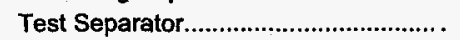 & 189.1 & 188.1 & 187.1 & 198.0 & 20,000 \\
\hline 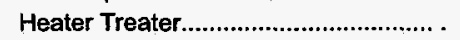 & 136.8 & 145.8 & 150.3 & 154.8 & 24,000 \\
\hline 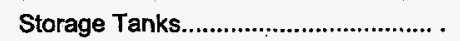 & 198.8 & 203.9 & 208.7 & 211.9 & 71,000 \\
\hline 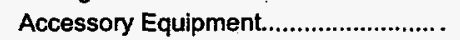 & 216.3 & 223.8 & 229.9 & 229.9 & 33,800 \\
\hline 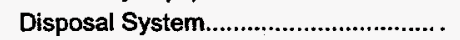 & 192.1 & 195.5 & 198.2 & 206.1 & 91,300 \\
\hline LACT Unit. & 174.2 & 171.0 & 171.0 & 171.0 & 15,900 \\
\hline 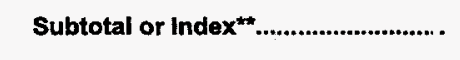 & 187.7 & 191.6 & 195.3 & 200.3 & 268,000 \\
\hline Total or Index & 182.0 & 192.7 & 199.3 & 224.2 & 976,500 \\
\hline
\end{tabular}


Table B3. Lease Equipment Costs and Indices for Primary Oil Production in South Texas (10 Wells Producing from 8,000 Feet by Gas Lift)

\begin{tabular}{|c|c|c|c|c|c|}
\hline \multirow{2}{*}{ Component } & \multicolumn{4}{|c|}{ Index $(1976=100)$} & \multirow{2}{*}{$\begin{array}{c}1997^{*} \\
\text { Cost } \\
\text { (dollars) }\end{array}$} \\
\hline & 1994 & 1995 & 1996 & 1997 & \\
\hline \multicolumn{6}{|l|}{ Producing Equipment: } \\
\hline 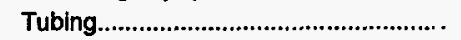 & 107.6 & 151,4 & 154.2 & 171.1 & 247,400 \\
\hline 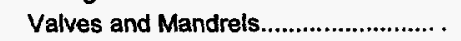 & 390.4 & 394.9 & 399.2 & 568.6 & 201,300 \\
\hline Pumping Equipment................................. & 162.2 & 168.2 & 174.1 & 209.8 & 221,300 \\
\hline Subtotal or Index $* * \ldots . . . . . . . . . . . . . . . . . . .$. & 162.8 & 187.8 & 191.9 & 234.7 & 6710,000 \\
\hline \multicolumn{6}{|l|}{ Gathering System: } \\
\hline 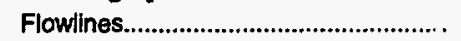 & 180.2 & 182.6 & 197.8 & 205.5 & 278,200 \\
\hline 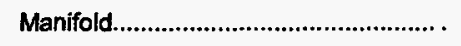 & 263.4 & 266.4 & 273.1 & 289.6 & 38,800 \\
\hline 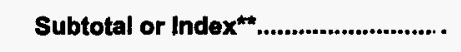 & 187.7 & 190.1 & 204.6 & 213.0 & 317,000 \\
\hline \multicolumn{6}{|l|}{ Lease Equipment: } \\
\hline 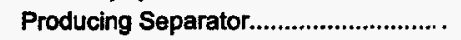 & 173.4 & 173.4 & 182.8 & 187.5 & 12,000 \\
\hline 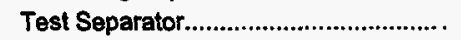 & 189.1 & 188.1 & 187.1 & 198.0 & 20,000 \\
\hline Heater Treater................................ . & 136.8 & 145.8 & 150.3 & 154.8 & 24,000 \\
\hline 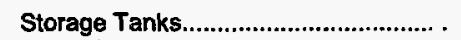 & 198.8 & 203.9 & 208.7 & 211.9 & 71,000 \\
\hline Accessory Equipment............................. & 216.3 & 223.8 & 229.9 & 229.9 & 33,800 \\
\hline 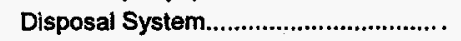 & 191.2 & 194.6 & 196.8 & 202.0 & 82,600 \\
\hline 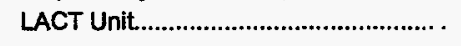 & 174.2 & 171.0 & 171.0 & 171.0 & 15,900 \\
\hline Subtotal or Index & 187.3 & 191.3 & 194.8 & 198.8 & 259,300 \\
\hline Total or index ${ }^{* 4}$ & 175.0 & 189.2 & 195.9 & 220.7 & $1,246,300$ \\
\hline
\end{tabular}

Table B4. Lease Equipment Costs and Indices for Primary Oll Production in South Texas (10 Wells Producing from 12,000 Feet by Hydraullc Lift)

\begin{tabular}{|c|c|c|c|c|c|}
\hline \multirow{2}{*}{ Component } & \multicolumn{4}{|c|}{ Index $(1976=100)$} & \multirow{2}{*}{$\begin{array}{c}1997^{*} \\
\text { Cost } \\
\text { (dollars) }\end{array}$} \\
\hline & 1994 & 1995 & 1996 & 1997 & \\
\hline \multicolumn{6}{|l|}{ Producing Equipment: } \\
\hline 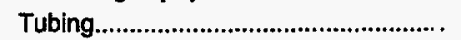 & 137.0 & 144.6 & 149.7 & 163.7 & 832,900 \\
\hline 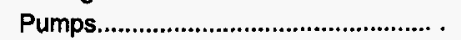 & 250.5 & 261.7 & 280.0 & 280.0 & 209,700 \\
\hline Pumping Equipment............................... . & 178.7 & 183.3 & 189.8 & 199.6 & 354,900 \\
\hline Subtotal or Index & 157.9 & 165.2 & 171.9 & 183.5 & $1,397,500$ \\
\hline \multicolumn{6}{|l|}{ Gathering System: } \\
\hline 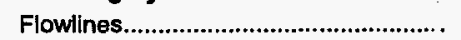 & 214.9 & 216.7 & 230.6 & 235.5 & 257,400 \\
\hline 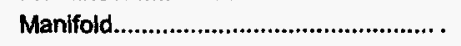 & 263.4 & 266.4 & 273.1 & 289.6 & 38,800 \\
\hline Subtotal or Index & 220.2 & 222.1 & 235.2 & 271.0 & 296,200 \\
\hline \multicolumn{6}{|l|}{ Lease Equipment: } \\
\hline Producing Separator................................ & 173.4 & 173.4 & 182.8 & 187.5 & 12,000 \\
\hline 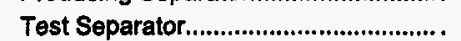 & 189.1 & 188.1 & 187.1 & 198.0 & 20,000 \\
\hline Heater Treater...................................... . & 136.8 & 145.8 & 150.3 & 154.8 & 24,000 \\
\hline 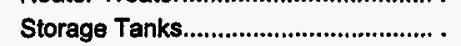 & 198.8 & 203.9 & 208.7 & 211.9 & 71,000 \\
\hline Accessory Equipment............................ & 216.3 & 223.8 & 229.9 & 229.9 & 33,1800 \\
\hline 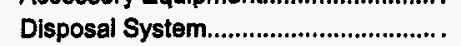 & 193.2 & 196.9 & 199.3 & 202.7 & 83,900 \\
\hline LACT Unit. & 174.2 & 171.0 & 171.0 & 171.0 & 15,900 \\
\hline Subtotal or Index & 187.9 & 192.0 & 195.6 & 199.1 & 260,300 \\
\hline Total or Index & 169.3 & 175.5 & 182.6 & 192.5 & $1,954,300$ \\
\hline
\end{tabular}


Table B5. Direct Annual Operating Costs and Indices for Primary Oil Production in South Texas (10 Wells Producing from 2,000 Feet by Rod Lift)

\begin{tabular}{|c|c|c|c|c|c|}
\hline \multirow{2}{*}{ Component } & \multicolumn{4}{|c|}{ Index $(1976=100)$} & \multirow{2}{*}{$\begin{array}{c}1997^{*} \\
\text { Cost } \\
\text { (dollars) }\end{array}$} \\
\hline & 1994 & 1995 & 1996 & 1997 & \\
\hline \multicolumn{6}{|l|}{ Normal Daily Expense: } \\
\hline 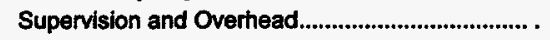 & 316.7 & 336.7 & 340.0 & 355.0 & 21,300 \\
\hline 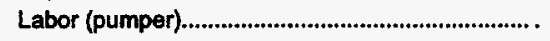 & 317.4 & 317.4 & 317.4 & 317.4 & 27,300 \\
\hline Auto Usage & 272.0 & 284.0 & 296.0 & 308.0 & 7,700 \\
\hline 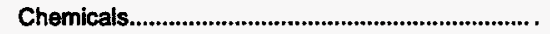 & 211.1 & 207.4 & 207.4 & 218.5 & 5,900 \\
\hline 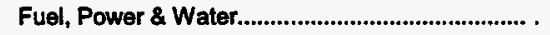 & 258.9 & 227.4 & 301.4 & 315.1 & 23,000 \\
\hline 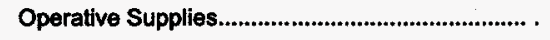 & 254.5 & 254.5 & 254.5 & 254.5 & 2,800 \\
\hline Subtotal or Index & 285.5 & 282.3 & 303.2 & 312.1 & $\mathbf{8 8 , 0 0 0}$ \\
\hline \multicolumn{6}{|l|}{ Surface Maintenance, Repair \& Services: } \\
\hline 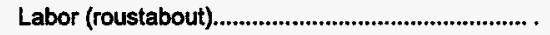 & 221.1 & 221.1 & 221.1 & 221.1 & 15,700 \\
\hline 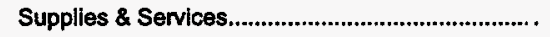 & 218.2 & 218.2 & 218.2 & 216.4 & 11,900 \\
\hline 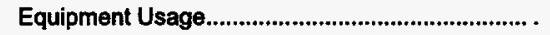 & 239.1 & 239.1 & 239.1 & 217.4 & 5,000 \\
\hline Other. & 316.7 & 316.7 & 316.7 & 300.0 & 3,600 \\
\hline Subtotal or Index & 229.8 & 229.8 & 229.8 & 224.8 & 36,200 \\
\hline \multicolumn{6}{|l|}{ Subsurface Maintenance, Repair \& Services: } \\
\hline 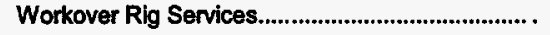 & 175.5 & 184.9 & 184.9 & 207.5 & 11,000 \\
\hline 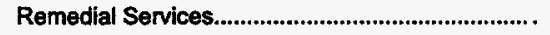 & 153.8 & 157.7 & 157.7 & 161.5 & 4,200 \\
\hline 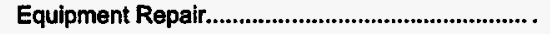 & 140.0 & 152.0 & 152.0 & 164.0 & 4,100 \\
\hline 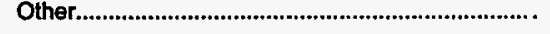 & 200.0 & 200.0 & 200.0 & 200.0 & 400 \\
\hline Subtotal or Index & 162.3 & 170.8 & 170.8 & 185.8 & 19,700 \\
\hline Total or Index & 245.4 & 245.4 & 256.1 & 262.1 & 143,900 \\
\hline
\end{tabular}

Table B6. Direct Annual Operating Costs and Indices for Primary Oil Production in South Texas (10 Wells Producing from 4,000 Feet by Gas Lift)

\begin{tabular}{|c|c|c|c|c|c|}
\hline \multirow{2}{*}{ Component } & \multicolumn{4}{|c|}{ Index $(1976=100)$} & \multirow{2}{*}{$\begin{array}{c}1997^{*} \\
\text { Cost } \\
\text { (dollars) }\end{array}$} \\
\hline & 1994 & 1995 & 1996 & 1997 & \\
\hline \multicolumn{6}{|l|}{ Normal Daily Expense: } \\
\hline Supervision and Overhead......................................... . & 316.2 & 335.3 & 339.7 & 355.9 & 24,200 \\
\hline Labor (pumper) & 317.4 & 317.4 & 317.4 & 317.4 & 27,300 \\
\hline 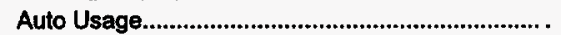 & 272.0 & 284.0 & 296.0 & 308.0 & 7,700 \\
\hline 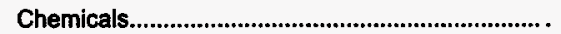 & 222.2 & 222.2 & 218.5 & 229.6 & 6,200 \\
\hline 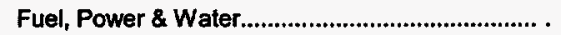 & 273.5 & 173.5 & 233.3 & 244.4 & 28,600 \\
\hline 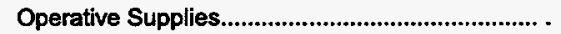 & 206.3 & 209.4 & 212.5 & 228.1 & 7,300 \\
\hline Subtotal or Index & 282.3 & 254.1 & 275.5 & 285.4 & 101,300 \\
\hline \multicolumn{6}{|l|}{ Surface Maintenance, Repair \& Services: } \\
\hline Labor (roustabout) & 221.1 & 221.1 & 221.1 & 221.1 & 15,700 \\
\hline 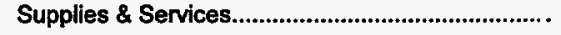 & 199.2 & 202.8 & 206.7 & 225.7 & 57,100 \\
\hline Equipment Usage & 237.5 & 237.5 & 237.5 & 216.7 & 5,200 \\
\hline 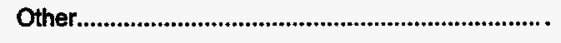 & 337.5 & 337.5 & 337.5 & 325.0 & 5,200 \\
\hline Subtotal or Index & 212.1 & 214.6 & 217.3 & 228.6 & $\mathbf{8 3 , 2 0 0}$ \\
\hline \multicolumn{6}{|l|}{ Subsurface Maintenance, Repair \& Services: } \\
\hline 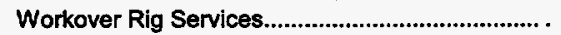 & 233.3 & 247,6 & 247.6 & 271.4 & 5,700 \\
\hline 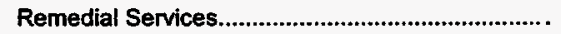 & 191.4 & 194.3 & 200.0 & 205.7 & 7,200 \\
\hline 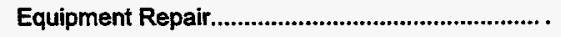 & 211.1 & 244.4 & 244.4 & 266.7 & 2,400 \\
\hline 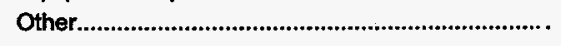 & 166.7 & 166.7 & 166.7 & 166.7 & 500 \\
\hline Subtotal or Index ${ }^{*+\ldots} \ldots$ & 205.9 & 216.2 & 219.1 & 232.4 & 15,800 \\
\hline Total or Index ${ }^{*+}$ & 243.2 & 232.5 & 243.7 & 254.5 & 200,300 \\
\hline
\end{tabular}


Table B7. Direct Annual Operating Costs and Indices for Primary Oil Production in South Texas (10 Wells Producing from 8,000 Feet by Gas Lift)

\begin{tabular}{|c|c|c|c|c|c|}
\hline \multirow{2}{*}{ Component } & \multicolumn{4}{|c|}{ Index $(1976=100)$} & \multirow{2}{*}{$\begin{array}{c}1997^{*} \\
\text { Cost } \\
\text { (dollars) }\end{array}$} \\
\hline & 1994 & 1995 & 1996 & 1997 & \\
\hline \multicolumn{6}{|l|}{ Normal Daily Expense: } \\
\hline 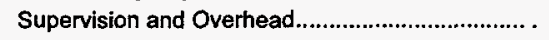 & 323.1 & 343.6 & 347.4 & 364.1 & 28,400 \\
\hline 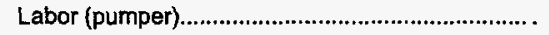 & 317.4 & 317.4 & 317.4 & 317.4 & 27,300 \\
\hline Auto Usage. & 272.0 & 284.0 & 296.0 & 308.0 & 7,700 \\
\hline 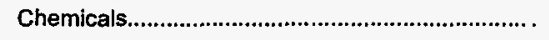 & 218.5 & 214.8 & 214.8 & 225.9 & 6,100 \\
\hline 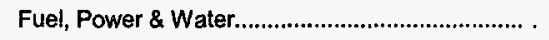 & 269.9 & 220.3 & 301.5 & 316.5 & $.42,100$ \\
\hline 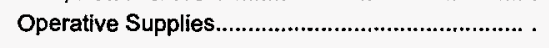 & 217.1 & 220.0 & 225.7 & 240.0 & 8,400 \\
\hline Subtotal or Index & 283.1 & 270.8 & 301.0 & 312.5 & 120,000 \\
\hline \multicolumn{6}{|l|}{ Surface Maintenance, Repair \& Services: } \\
\hline Labor (roustabout) & 221.1 & 221.1 & 221.1 & 221.1 & 15,700 \\
\hline 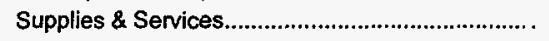 & 214.9 & 219.2 & 223.5 & 244.5 & 68,700 \\
\hline 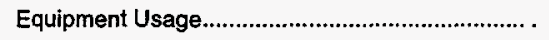 & 248.0 & 248.0 & 248.0 & 228.0 & 5,700 \\
\hline 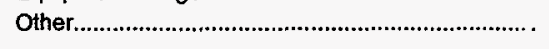 & 315.0 & 315.0 & 315.0 & 300.0 & 6,000 \\
\hline Subtotal or Index & 223.2 & 226.2 & 229.2 & 242.1 & 96,100 \\
\hline \multicolumn{6}{|l|}{ Subsurface Maintenance, Repair \& Services: } \\
\hline 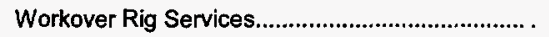 & 224.2 & 236.4 & 236.4 & 254.5 & 8,400 \\
\hline 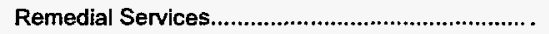 & 160.0 & 164.4 & 166.7 & 172.2 & 15,500 \\
\hline 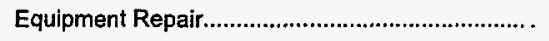 & 193.3 & 226.7 & 226.7 & 253.3 & 3,800 \\
\hline 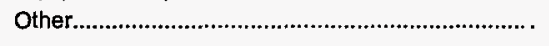 & 166.7 & 177.8 & 177.8 & 177.8 & 1,600 \\
\hline Subtotal or Index & 178.2 & 187.8 & 189.1 & 199.3 & 29,300 \\
\hline Total or Index & 240.8 & 238.6 & 252.6 & 264.4 & 245,400 \\
\hline
\end{tabular}

Table B8. Direct Annual Operating Costs and Indices for Primary Oil Production in South Texas (10 Wells Producing from 12,000 Feet by Hydraulic Lift)

\begin{tabular}{|c|c|c|c|c|c|}
\hline \multirow{2}{*}{ Component } & \multicolumn{4}{|c|}{ Index $(1976=100)$} & \multirow{2}{*}{$\begin{array}{c}1997^{*} \\
\text { Cost } \\
\text { (dollars) }\end{array}$} \\
\hline & 1994 & 1995 & 1996 & 1997 & \\
\hline \multicolumn{6}{|l|}{ Normal Daily Expense: } \\
\hline 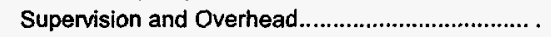 & 320.4 & 341.8 & 344.9 & 361.2 & 35,400 \\
\hline 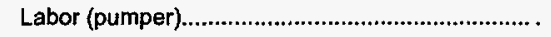 & 317.4 & 317.4 & 317.4 & 317.4 & 27,300 \\
\hline 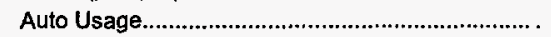 & 272.0 & 284.0 & 296.0 & 308.0 & 7,700 \\
\hline 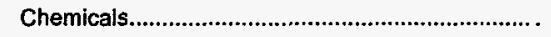 & 206.5 & 206.5 & 206.5 & 216.1 & 6,700 \\
\hline Fuel, Power \& Water........................................... . & 244.4 & 213.4 & 295.2 & 310.2 & 58,000 \\
\hline Operative Supplies............................................. . & 300.0 & 300.0 & 300.0 & 300.0 & 4,200 \\
\hline Subtotal or Index & 276.2 & 268.5 & 304.5 & 315.9 & 139,300 \\
\hline \multicolumn{6}{|l|}{ Surface Maintenance, Repair \& Services: } \\
\hline 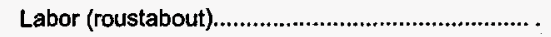 & 221.1 & 221.1 & 221.1 & 221.1 & 15,700 \\
\hline 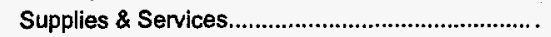 & 215.8 & 216.7 & 217.5 & 221.7 & 26,600 \\
\hline Equipment Usage & 248.0 & 248.0 & 248.0 & 228.0 & 5,700 \\
\hline 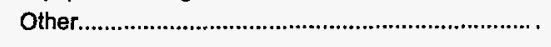 & 216.7 & 216.7 & 216.7 & 233.3 & 1,400 \\
\hline Subtotal or Index & 221.2 & 221.6 & 222.1 & 222.5 & 49,400 \\
\hline \multicolumn{6}{|l|}{ Subsurface Maintenance, Repair \& Services: } \\
\hline Workover Rig Services............................................ & 195.7 & 201.4 & 201.4 & 212.9 & 14,900 \\
\hline 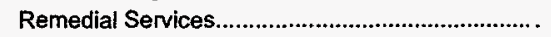 & 240.9 & 245.0 & 247.6 & 257.6 & $6 \mathrm{~S}, 300$ \\
\hline 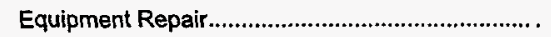 & 403.4 & 421.2 & 450.8 & 450.8 & 80,700 \\
\hline 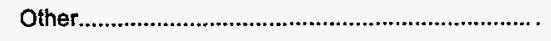 & 161.5 & 161.5 & 161.5 & 169.2 & 2,200 \\
\hline 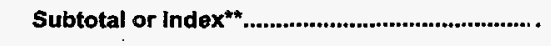 & 287.8 & 296.6 & 307.9 & 314.7 & 167,100 \\
\hline Total or Index & 271.1 & 272.3 & 290.7 & 298.0 & 355,800 \\
\hline
\end{tabular}


Table C1. Lease Equipment Costs and Indices for Primary Oil Production in South Louisiana (10 Wells Producing from 2,000 Feet by Rod Lift)

\begin{tabular}{|c|c|c|c|c|c|}
\hline \multirow{2}{*}{ Component } & \multicolumn{4}{|c|}{ Index $(1976=100)$} & \multirow{2}{*}{$\begin{array}{c}1997^{\star} \\
\text { Cost } \\
\text { (dollars) }\end{array}$} \\
\hline & 1994 & 1995 & 1996 & 1997 & \\
\hline \multicolumn{6}{|l|}{ Producing Equipment: } \\
\hline 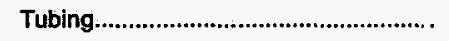 & 110.2 & 155.0 & 158.1 & 175.4 & 61,900 \\
\hline 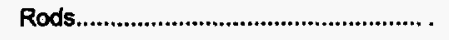 & 123.0 & 123.0 & 123.0 & 127.0 & 25,900 \\
\hline 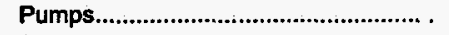 & 147.6 & 152.4 & 157.1 & 166.7 & 14,000 \\
\hline Pumping Equipment.................................. & 179.1 & 191.4 & 203.2 & 237.4 & 305,100 \\
\hline Subtotal or Index & 159.1 & 175.8 & 184.4 & 211.3 & 406,900 \\
\hline \multicolumn{6}{|l|}{ Gathering System: } \\
\hline 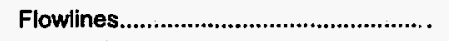 & 263.0 & 269.1 & 294.5 & 315.8 & 104,200 \\
\hline 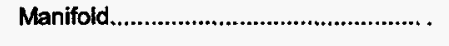 & 264.7 & 267.7 & 274.4 & 291.0 & 38,700 \\
\hline Subtotal or Index & 263.5 & 268.7 & 288.8 & 308.6 & 142,900 \\
\hline \multicolumn{6}{|l|}{ Lease Equipment: } \\
\hline Producing Separator................................. & 173.4 & 173.4 & 182.8 & 187.5 & 12,000 \\
\hline 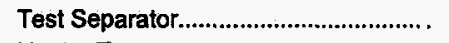 & 189.1 & 188.1 & 187.1 & 198.0 & 20,000 \\
\hline 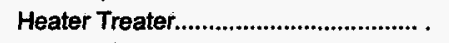 & 136.8 & 145.8 & 150.3 & 154.8 & 24,000 \\
\hline 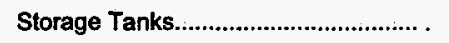 & 196.7 & 203.3 & 209.9 & 218.8 & 73,300 \\
\hline Accessory Equipment............................... & 212.9 & 220.4 & 227.2 & 227.9 & 33,500 \\
\hline Disposal System & 184.9 & 189.7 & 195.9 & 208.4 & 87,100 \\
\hline 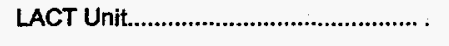 & 174.2 & 171.0 & 171.0 & 171.0 & 15,900 \\
\hline Subtotal or Index & 184.4 & 189.2 & 194.5 & 202.4 & 265,800 \\
\hline Total or Index ${ }^{\text {t+n }}$ & 181.1 & 192.2 & 201.1 & 220.3 & 815,600 \\
\hline
\end{tabular}

Table C2. Lease Equipment Costs and Indices for Primary Oil Production in South Louisiana ( 10 Wells Producing from 4,000 Feet by Gas Lift)

\begin{tabular}{|c|c|c|c|c|c|}
\hline \multirow{2}{*}{ Component } & \multicolumn{4}{|c|}{ Index $(1976=100)$} & \multirow{2}{*}{$\begin{array}{c}1997^{*} \\
\text { Cost } \\
\text { (dollars) }\end{array}$} \\
\hline & 1994 & 1995 & 1996 & 1997 & \\
\hline \multicolumn{6}{|l|}{ Producing Equipment: } \\
\hline Tubing & 107.4 & 151.5 & 154.3 & 171.3 & 122,800 \\
\hline 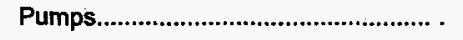 & 390.1 & 394.5 & 399.2 & 568.4 & 143,800 \\
\hline Pumping Equipment................................ . & 163.9 & 169.9 & 176.0 & 212.0 & 221,300 \\
\hline Subtotal or Index & 172.2 & 191.6 & 196.3 & 242.3 & 487,900 \\
\hline \multicolumn{6}{|l|}{ Gathering System: } \\
\hline 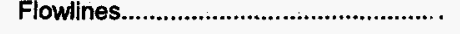 & 242.8 & 251.0 & 274.2 & 294.7 & 231,900 \\
\hline 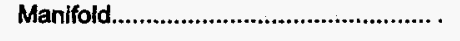 & 264.7 & 267.7 & 274.4 & 291.0 & 38,700 \\
\hline 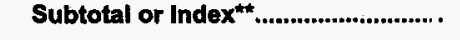 & 246.0 & 253.4 & 274.2 & 294.1 & 270,600 \\
\hline \multicolumn{6}{|l|}{ Lease Equipment: } \\
\hline Producing Separator................................. & 173.4 & 173.4 & 182.8 & 187.5 & 12,000 \\
\hline 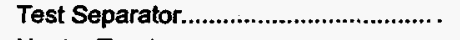 & 189.1 & 188.1 & 187.1 & 198.0 & 20,000 \\
\hline 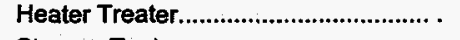 & 136.8 & 145.8 & 150.3 & 154.8 & 24,000 \\
\hline 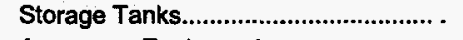 & 196.7 & 203.3 & 209.9 & 218.8 & 73,300 \\
\hline Accessory Equipment................................ & 212.9 & 220.4 & 227.2 & 227.9 & 33,500 \\
\hline Disposal System & 185.6 & 190.2 & 197.0 & 209.5 & 90,100 \\
\hline 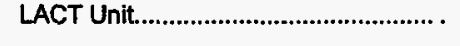 & 174.2 & 171.0 & 171.0 & 171.0 & 15,900 \\
\hline 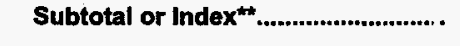 & 184.6 & 189.4 & 194.9 & 202.9 & 268,800 \\
\hline Total or Index & 192.0 & 204.2 & 212.7 & 241.2 & $1,027,300$ \\
\hline
\end{tabular}


Table C3. Lease Equipment Costs and Indices for Primary Oil Production in South Louisiana (10 Wells Producing from 8,000 Feet by Gas Lift)

\begin{tabular}{|c|c|c|c|c|c|}
\hline \multirow{2}{*}{ Component } & \multicolumn{4}{|c|}{ Index $(1976=100)$} & \multirow{2}{*}{$\begin{array}{c}\text { 1997* } \\
\text { Cost } \\
\text { (dollars) }\end{array}$} \\
\hline & 1994 & 1995 & 1996 & 1997 & \\
\hline \multicolumn{6}{|l|}{ Producing Equipment: } \\
\hline 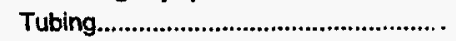 & 107.5 & 151.7 & 154.5 & 171.5 & 245,600 \\
\hline 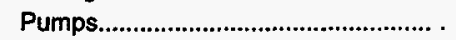 & 390.4 & 394.9 & 399.2 & 568.6 & 201,300 \\
\hline Pumping Equipment............................... & 162.2 & 168.2 & 174.1 & 209.8 & 221,300 \\
\hline Subtotal or Index $* * \ldots . . . . . . . . . . . . . . . . . . . . . .$. & 163.1 & 188.1 & 192.3 & 235.2 & 668,200 \\
\hline \multicolumn{6}{|l|}{ Gathering System: } \\
\hline 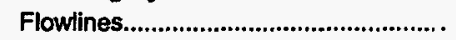 & 238.8 & 247.4 & 270.3 & 290.5 & 358,800 \\
\hline 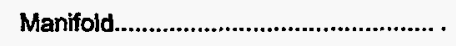 & 264.7 & 267.7 & 274.4 & 291.0 & 38,700 \\
\hline 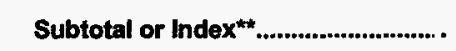 & 241.3 & 249.4 & 270.7 & 290.6 & 397,500 \\
\hline \multicolumn{6}{|l|}{ Lease Equipment: } \\
\hline 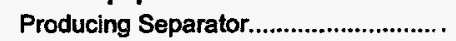 & 173.4 & 173.4 & 182.8 & 187.5 & 12,000 \\
\hline 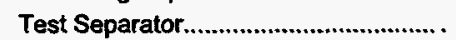 & 189.1 & 188.1 & 187.1 & 198.0 & 20,000 \\
\hline Heater Treater...................................... . & 136.8 & 145.8 & 150.3 & 154.8 & $2,4,000$ \\
\hline Storage Tanks....................................... . & 196.7 & 203.3 & 209.9 & 218.8 & 73,300 \\
\hline Accessory Equipment............................. & 212.9 & 220.4 & 227.2 & 227.9 & 33,500 \\
\hline 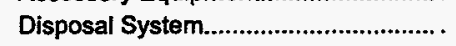 & 191.4 & 197.8 & 206.2 & 217.0 & $8: 3,100$ \\
\hline 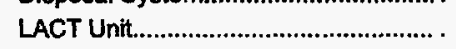 & 174.2 & 171.0 & 171.0 & 171.0 & 15,900 \\
\hline 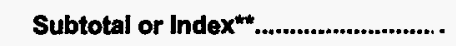 & 186.4 & 191.7 & 197.7 & 205.1 & 266,800 \\
\hline Total or Index ${ }^{* *}$ & 188.0 & 204.2 & 213.0 & 241.8 & $1,332,500$ \\
\hline \multicolumn{6}{|c|}{$\begin{array}{l}\text { Table C4. Lease Equipment Costs and Indices for Primary Oil Production in South Louisiana } \\
\text { (10 Wells Producing from } 12,000 \text { Feet by Hydraulic Lift) }\end{array}$} \\
\hline \multirow[b]{2}{*}{ Component } & \multicolumn{4}{|c|}{ Index $(1976=100)$} & \multirow{2}{*}{$\begin{array}{c}1997^{*} \\
\text { Cosit } \\
\text { (dollats) }\end{array}$} \\
\hline & 1994 & 1995 & 1996 & 1997 & \\
\hline \multicolumn{6}{|l|}{ Producing Equipment: } \\
\hline 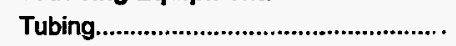 & 137.3 & 144.9 & 150.0 & 164.1 & 828,100 \\
\hline 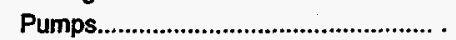 & 250.5 & 261.7 & 280.0 & 280.0 & 209,700 \\
\hline Pumping Equipment................................. & 173.8 & 179.2 & 186.5 & 203.7 & 362,200 \\
\hline Subtotal or Index ${ }^{* *} \ldots . . . . . . . . . . . . . . . . . . . . . .$. & 157.1 & 164.5 & 171.5 & 184.9 & $1,400,000$ \\
\hline \multicolumn{6}{|l|}{ Gathering System: } \\
\hline 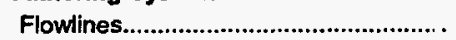 & 207.8 & 215.3 & 232.7 & 249.7 & 330,600 \\
\hline 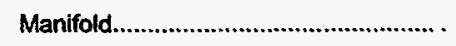 & 264.7 & 267.7 & 274.4 & 291.0 & 38,700 \\
\hline 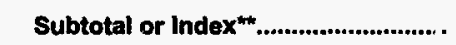 & 213.0 & 220.0 & 236.5 & 253.5 & 369,300 \\
\hline \multicolumn{6}{|l|}{ Lease Equipment: } \\
\hline Producing Separator................................. & 173.4 & 173.4 & 182.8 & 187.5 & 12,000 \\
\hline 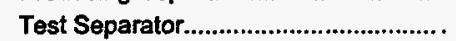 & 189.1 & 188.1 & 187.1 & 198.0 & 20,000 \\
\hline Heater Treater...................................... . & 136.8 & 145.8 & 150.3 & 154.8 & 24,000 \\
\hline 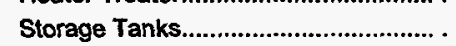 & 196.7 & 203.3 & 209.9 & 218.8 & 73,300 \\
\hline 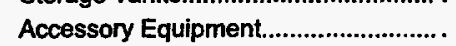 & 212.9 & 220.4 & 227.2 & 227.9 & 33,500 \\
\hline Disposal System & 204.5 & 210.3 & 218.8 & 231.4 & 92,100 \\
\hline 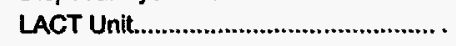 & 174.2 & 171.0 & 171.0 & 171.0 & 15,900 \\
\hline Subtotal or Index ${ }^{*+} \ldots$ & 190.4 & 195.5 & 201.5 & 209.4 & 270,800 \\
\hline Total or Index & 169.1 & 176.3 & 184.4 & 197.6 & $2,040,100$ \\
\hline
\end{tabular}


Table C5. Direct Annual Operating Costs and Indices for Primary Oil Production in South Louislana (10 Wells Producing from 2,000 Feet by Rod Lift)

\begin{tabular}{|c|c|c|c|c|c|}
\hline \multirow{2}{*}{ Component } & \multicolumn{4}{|c|}{ Index $(1976=100)$} & \multirow{2}{*}{$\begin{array}{c}1997^{*} \\
\text { Cost } \\
\text { (dollars) }\end{array}$} \\
\hline & 1994 & 1995 & 1996 & 1997 & \\
\hline \multicolumn{6}{|l|}{ Normal Daily Expense: } \\
\hline 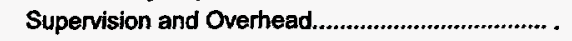 & 305.0 & 323.3 & 326.7 & 341.7 & 20,500 \\
\hline 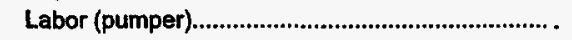 & 257.0 & 261.0 & 265.1 & 295.9 & 50,900 \\
\hline 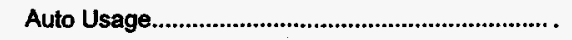 & 272.0 & 284.0 & 296.0 & 308.0 & 7,700 \\
\hline 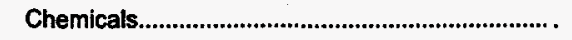 & 203.7 & 203.7 & 203.7 & 218.5 & 5,900 \\
\hline Fuel, Power \& Water................................................. . & 376.0 & 292.0 & 418.0 & 438.0 & 21,900 \\
\hline Operative Supplies............................................... . & 228.6 & 228.6 & 228.6 & 257.1 & 1,800 \\
\hline Subtotal or Index $* * \ldots$ & 279.2 & 273.0 & 295.0 & 318.8 & 108,700 \\
\hline \multicolumn{6}{|l|}{ Surface Maintenance, Repair \& Services: } \\
\hline Labor (roustabout) & 205.3 & 210.5 & 215.8 & 244.7 & $\mathbf{9 , 3 0 0}$ \\
\hline 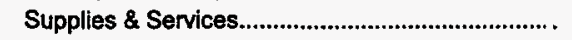 & 216.7 & 222.2 & 227.8 & 255.6 & 9,200 \\
\hline 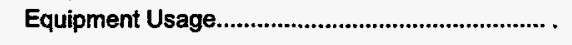 & 331.3 & 350.0 & 350.0 & 393.8 & 6,300 \\
\hline Subtotal or Index $+*+\ldots$ & 232.2 & 240.0 & 244.4 & 275.6 & 24,800 \\
\hline \multicolumn{6}{|l|}{ Subsurface Maintenance, Repair \& Services: } \\
\hline 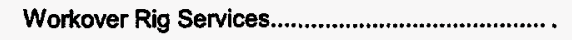 & 206.7 & 206.7 & 206.7 & 225.0 & 13,500 \\
\hline 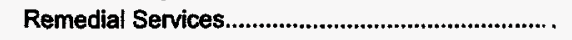 & 142.3 & 146.2 & 150.0 & 153.8 & 4,000 \\
\hline 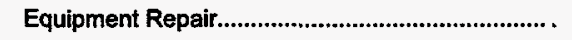 & 144.0 & 152.0 & 156.0 & 168.0 & 4,200 \\
\hline 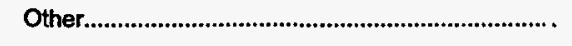 & 200.0 & 200.0 & 200.0 & 200.0 & 400 \\
\hline Subtotal or Index & 177.9 & 180.5 & 182.3 & 195.6 & 22,100 \\
\hline 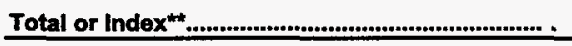 & 250.4 & 248.3 & 263.2 & 286.0 & 155,600 \\
\hline \multicolumn{6}{|c|}{$\begin{array}{l}\text { Table C6. Direct Annual Operating Costs and Indices for Primary Oil Production in South Louislana } \\
\text { (10 Wells Producing from 4,000 Feet by Gas Lift) }\end{array}$} \\
\hline \multirow[b]{2}{*}{ Component } & \multicolumn{4}{|c|}{ Index $(1976=100)$} & $1997^{*}$ \\
\hline & 1994 & 1995 & 1996 & 1997 & $\begin{array}{c}\text { Cost } \\
\text { (dollars) }\end{array}$ \\
\hline \multicolumn{6}{|l|}{ Normal Daily Expense: } \\
\hline 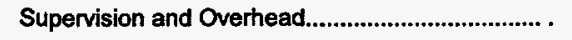 & 305.9 & 325.0 & 327.9 & 342.6 & 23,300 \\
\hline 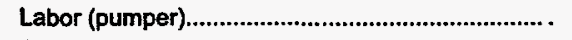 & 257.0 & 261.0 & 265.1 & 295.9 & 50,900 \\
\hline 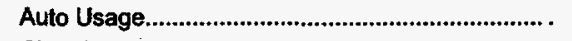 & 272.0 & 284.0 & 296.0 & 308.0 & 7,700 \\
\hline 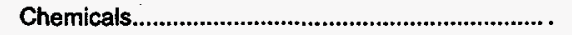 & 214.8 & 214.8 & 214.8 & 229.6 & 6,200 \\
\hline 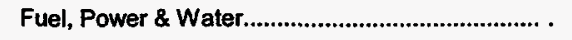 & 425.3 & 326.6 & 473.4 & 497.5 & 39,300 \\
\hline 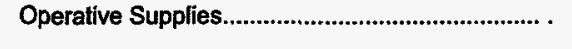 & 196.6 & 203.4 & 206.9 & 244.8 & 7,100 \\
\hline Subtotal or Index & 292.3 & 279.0 & 311.3 & 336.3 & 134,500 \\
\hline \multicolumn{6}{|l|}{ Surface Maintenance, Repair \& Services: } \\
\hline 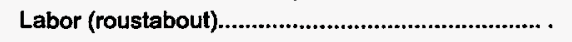 & 205.3 & 210.5 & 215.8 & 244.7 & 9,300 \\
\hline 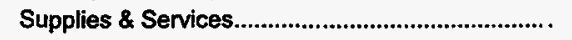 & 193.7 & 199.6 & 205.5 & 245.5 & 62,100 \\
\hline 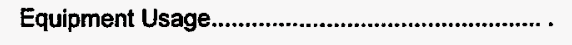 & 323.5 & 341.2 & 341.2 & 388.2 & 6,600 \\
\hline 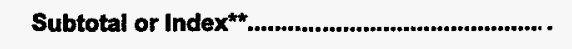 & 202.3 & 208.8 & 214.3 & 253.2 & 78,000 \\
\hline \multicolumn{6}{|l|}{ Subsurface Maintenance, Repair \& Services: } \\
\hline 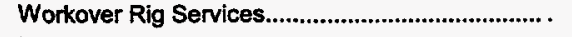 & 211.1 & 211.1 & 211.1 & 225.9 & 6,100 \\
\hline 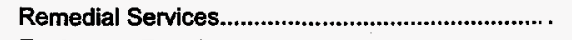 & 160.0 & 162.9 & 165.7 & 171.4 & 6,000 \\
\hline 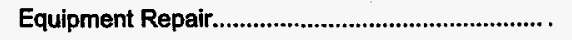 & 211.1 & 244.4 & 244.4 & 266.7 & 2,400 \\
\hline 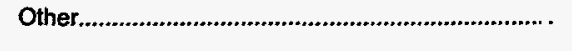 & 166.7 & 166.7 & 166.7 & 166.7 & 500 \\
\hline Subtotal or Index & 185.1 & 190.5 & 191.9 & 202.7 & 15,000 \\
\hline (.......................................................... . . & 246.7 & 243.0 & 261.8 & 290.9 & 227,500 \\
\hline
\end{tabular}


Table C7. Direct Annual Operating Costs and Indices for Primary Oil Production in South Louisiana (10 Wells Producing from 8,000 Feet by Gas Lift)

\begin{tabular}{|c|c|c|c|c|c|}
\hline \multirow{2}{*}{ Component } & \multicolumn{4}{|c|}{ Index $(1976=100)$} & \multirow{2}{*}{$\begin{array}{c}1997^{*} \\
\text { Cost } \\
\text { (dollars) }\end{array}$} \\
\hline & 1994 & 1995 & 1996 & 1997 & \\
\hline \multicolumn{6}{|l|}{ Normal Dally Expense: } \\
\hline 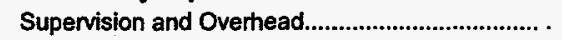 & 310.1 & 330.4 & 332.9 & 349.4 & 27,600 \\
\hline Labor (pumper) & 257.0 & 261.0 & 265.1 & 295.9 & 50,900 \\
\hline Auto Usage & 272.0 & 284.0 & 296.0 & 308.0 & 7,700 \\
\hline 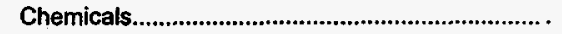 & 259.1 & 259.1 & 259.1 & 277.3 & 6,100 \\
\hline 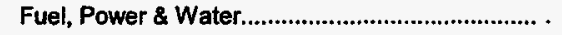 & 422.2 & 324.4 & 471.1 & 495.6 & 44,600 \\
\hline 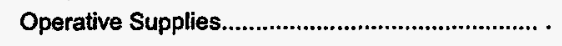 & 212.5 & 218.8 & 225.0 & 268.8 & 8,600 \\
\hline Subtotal or Index & 300.0 & 285.7 & 320.5 & 346.4 & 145,500 \\
\hline \multicolumn{6}{|l|}{ Surface Maintenance, Repair \& Services: } \\
\hline Labor (roustabout). & 205.3 & 210.5 & 215.8 & 244.7 & 9,300 \\
\hline 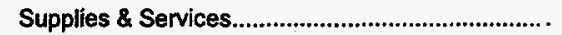 & 213.9 & 220.3 & 226.7 & 271.5 & 76,300 \\
\hline Equipment Usage & 344.4 & 361.1 & 366.7 & 416.7 & 7,500 \\
\hline Subtotal or Index & 219.9 & 226.7 & 232.9 & 276.3 & 93,100 \\
\hline \multicolumn{6}{|l|}{ Subsurface Maintenance, Repair \& Services: } \\
\hline 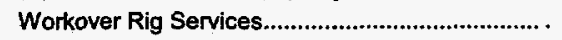 & 287.2 & 287.2 & 287.2 & 310.3 & 12,100 \\
\hline 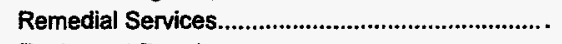 & 158.9 & 160.0 & 162.2 & 172.2 & 15,500 \\
\hline 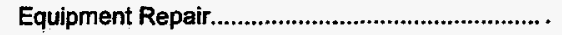 & 207.1 & 242.9 & 242.9 & 271.4 & 3,800 \\
\hline Other & 166.7 & 177.8 & 177.8 & 177.8 & 1,600 \\
\hline Subtotal or index & 196.7 & 201.3 & 202.6 & 217.1 & 33,000 \\
\hline Total or Index ${ }^{*+} \ldots \ldots$ & 253.0 & 249.7 & 268.3 & 298.8 & 271,600 \\
\hline
\end{tabular}

Table C8. Direct Annual Operating Costs and Indices for Primany Oil Production in South Louisiana (10 Wells Producing from 12,000 Feet by Hydraulic Lift)

\begin{tabular}{|c|c|c|c|c|c|}
\hline \multirow{2}{*}{ Component } & \multicolumn{4}{|c|}{ Index $(1976=100)$} & \multirow{2}{*}{$\begin{array}{c}1997^{\star} \\
\text { Cost } \\
\text { (dollars) }\end{array}$} \\
\hline & 1994 & 1995 & 1996 & 1997 & \\
\hline \multicolumn{6}{|l|}{ Normal Daily Expense: } \\
\hline Supervision and Overhead.................................... & 313.3 & 334.7 & 336.7 & 354.1 & 34,700 \\
\hline Labor (pumper) & 257.0 & 261.0 & 265.1 & 295.9 & 50,900 \\
\hline Auto Usage & 272.0 & 284.0 & 296.0 & 308.0 & 7,700 \\
\hline 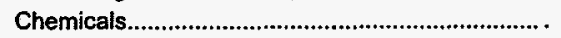 & 196.4 & 196.4 & 196.4 & 210.7 & 5,900 \\
\hline 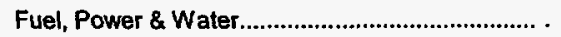 & 415.3 & 317.8 & 462.7 & 488.1 & 57,600 \\
\hline Operative Supplies................................................... . & 214.3 & 214.3 & 221.4 & 235.7 & 3,300 \\
\hline 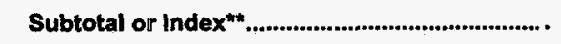 & 305.9 & 287.5 & 327.9 & 351.9 & 160,100 \\
\hline \multicolumn{6}{|l|}{ Surface Maintenance, Repair \& Services: } \\
\hline 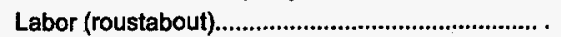 & 205.3 & 210.5 & 215.8 & 244.7 & 9,300 \\
\hline 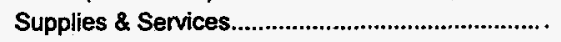 & 206.6 & 209.4 & 211.3 & 227.4 & 24,100 \\
\hline Equipment Usage................................................... . & 344.4 & 361.1 & 366.7 & 416.7 & 7,500 \\
\hline Subtotal or Index & 221.6 & 226.5 & 229.6 & 252.5 & 40,900 \\
\hline \multicolumn{6}{|l|}{ Subsurface Maintenance, Repair \& Services: } \\
\hline Workover Rig Services.............................................. & 193.4 & 193.4 & 193.4 & 202.6 & 15,400 \\
\hline 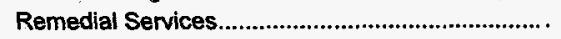 & 183.3 & 184.4 & 185.5 & 199.3 & 53,600 \\
\hline 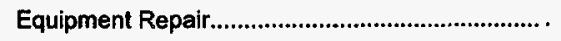 & 423.0 & 441.4 & 472.4 & 472.4 & 82,200 \\
\hline 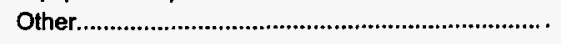 & 175.0 & 175.0 & 175.0 & 183.3 & 2,200 \\
\hline Subtotal or Index"*................................................ & 263.1 & 269.7 & 280.4 & 288.9 & 153,400 \\
\hline Total or Index ${ }^{* * \ldots} .$. & 274.2 & 270.6 & 292.1 & 308.7 & 354,400 \\
\hline
\end{tabular}


Table D1. Lease Equipment Costs and Indices for Primary Oil Production in Oklahoma (10 Wells Producing from 2,000 Feet by Rod Lift)

\begin{tabular}{|c|c|c|c|c|c|}
\hline \multirow{2}{*}{ Component } & \multicolumn{4}{|c|}{ Index $(1976=100)$} & \multirow{2}{*}{$\begin{array}{c}1997^{*} \\
\text { Cost } \\
\text { (dollars) }\end{array}$} \\
\hline & 1994 & 1995 & 1996 & 1997 & \\
\hline \multicolumn{6}{|l|}{ Producing Equipment: } \\
\hline 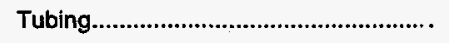 & 109.3 & 154.0 & 157.1 & 174.3 & 61,700 \\
\hline 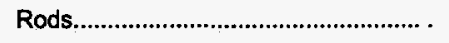 & 112.3 & 112.3 & 113.2 & 114.7 & 23,400 \\
\hline Pumps....................................... & 147.6 & 152.4 & 157.1 & 166.7 & 14,000 \\
\hline Pumping Equipment.............................. . & 176.1 & 188.3 & 199.8 & 234.1 & 305,300 \\
\hline 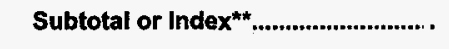 & 156.0 & 172.5 & 181.1 & 207.8 & 404,400 \\
\hline \multicolumn{6}{|l|}{ Gathering System: } \\
\hline 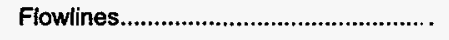 & 259.5 & 264.3 & 271.4 & 278.6 & 35,100 \\
\hline 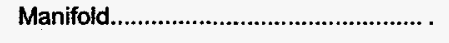 & 264.4 & 268.2 & 274.2 & 290.9 & 38,400 \\
\hline 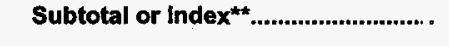 & 262.0 & 266.3 & 272.9 & 284.9 & 73,500 \\
\hline \multicolumn{6}{|l|}{ Lease Equipment: } \\
\hline Producing Separator.................................... & 173.4 & 173.4 & 182.8 & 187.5 & 12,000 \\
\hline 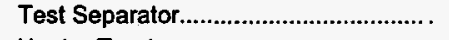 & 189.1 & 188.1 & 187.1 & 198.0 & 20,000 \\
\hline Heater Treater.......................................... . & 136.8 & 145.8 & 150.3 & 154.8 & 24,000 \\
\hline 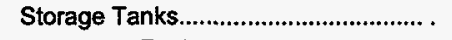 & 195.2 & 200.9 & 205.4 & 215.5 & 72,200 \\
\hline Accessory Equipment............................... & 212.9 & 220.4 & 227.2 & 227.9 & 33,500 \\
\hline 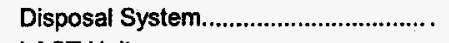 & 184.0 & 187.7 & 191.7 & 202.0 & 80,600 \\
\hline 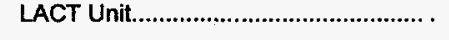 & 174.2 & 171.0 & 171.0 & 171.0 & 15,900 \\
\hline Subtotal or Index $x^{\star \hbar}$ & 183.7 & 187.9 & 192.0 & 199.5 & 258,200 \\
\hline 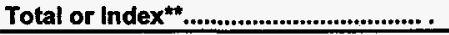 & 174.1 & 185.1 & 191.9 & 210.4 & 736,100 \\
\hline
\end{tabular}

Table D2. Lease Equipment Costs and Indices for Primary Oil Production in Oklahoma ( 10 Wells Producing from 4,000 Feet by Rod Lift)

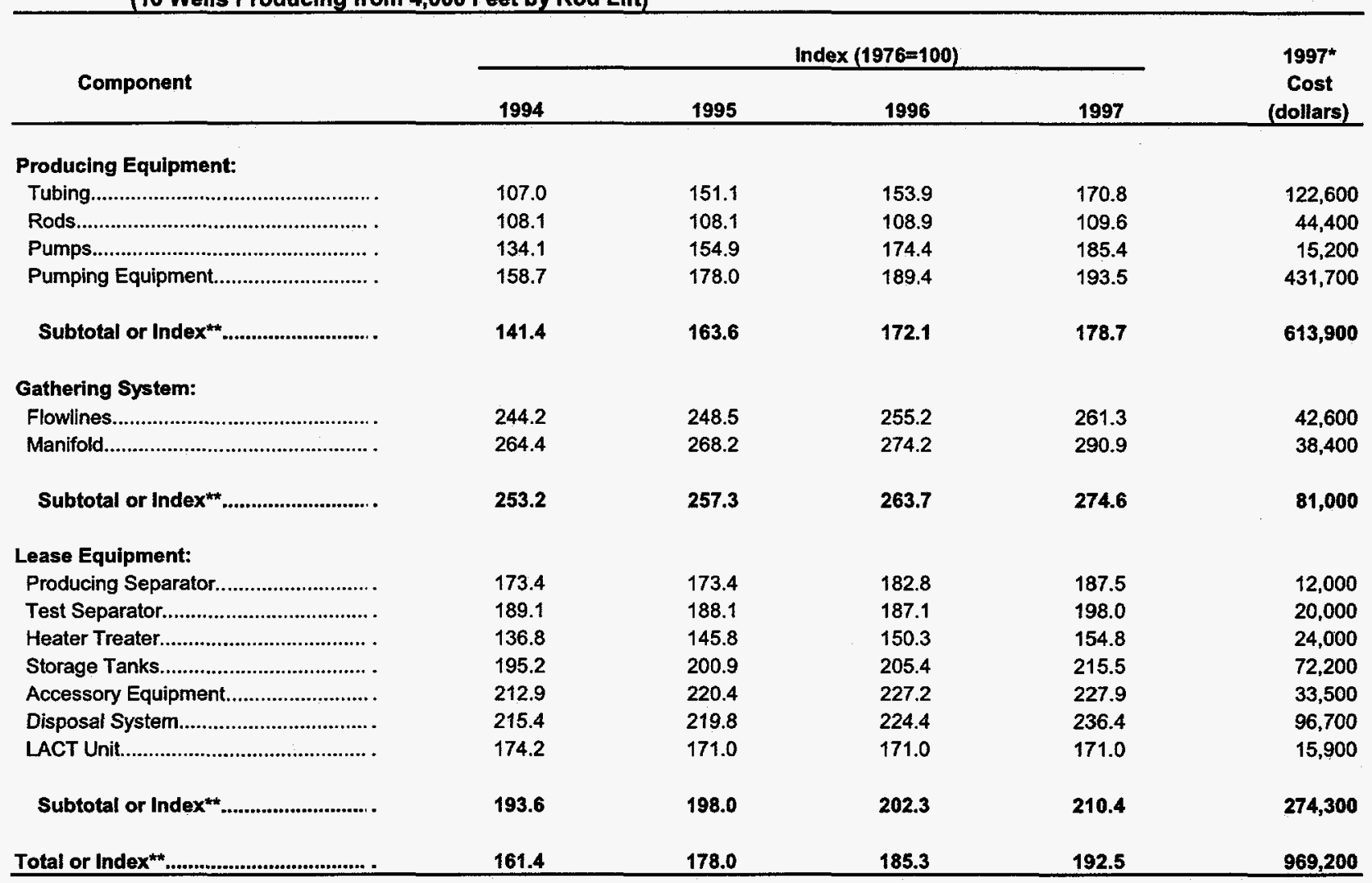


Table D3. Lease Equipment Costs and Indices for Primary Oil Production in Oklahoma (10 Wells Producing from 8,000 Feet by Hydraulic Lift)

\begin{tabular}{|c|c|c|c|c|c|}
\hline \multirow{2}{*}{ Component } & \multicolumn{4}{|c|}{ Index $(1976=100)$} & \multirow{2}{*}{$\begin{array}{c}1997^{\prime \prime} \\
\text { Cost } \\
\text { (dollars) }\end{array}$} \\
\hline & 1994 & 1995 & 1996 & 1997 & \\
\hline \multicolumn{6}{|l|}{ Producing Equipment: } \\
\hline 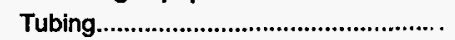 & 133.2 & 163.8 & 169.8 & 188.6 & 537,200 \\
\hline 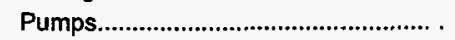 & 250.5 & 261.7 & 280.0 & 280.0 & 209,700 \\
\hline Pumping Equipment.................................. & 183.9 & 189.0 & 196.5 & 213.5 & 326,300 \\
\hline Subtotal or Index ${ }^{* *} \ldots$ & 165.5 & 185.6 & 193.9 & 209.4 & $1,073,200$ \\
\hline \multicolumn{6}{|l|}{ Gathering System: } \\
\hline 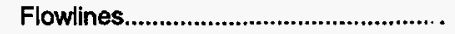 & 193.1 & 197.0 & 202.3 & 203.2 & 150,600 \\
\hline 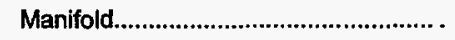 & 264.4 & 268.2 & 274.2 & 290.9 & 38,400 \\
\hline Subtotal or Index ${ }^{\star \star *}$ & 203.9 & 207.8 & 213.2 & 216.5 & 189,1000 \\
\hline \multicolumn{6}{|l|}{ Lease Equipment: } \\
\hline Producing Separator.................................. & 173.4 & 173.4 & 182.8 & 187.5 & 12,000 \\
\hline 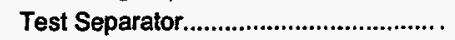 & 189.1 & 188.1 & 187.1 & 198.0 & 20,000 \\
\hline 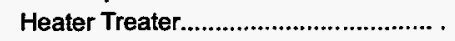 & 136.8 & 145.8 & 150.3 & 154.8 & 24,000 \\
\hline 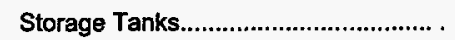 & 195.2 & 200.9 & 205.4 & 215.5 & 72,200 \\
\hline 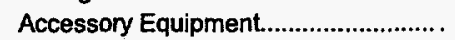 & 212.9 & 220.4 & 227.2 & 227.9 & 33,500 \\
\hline 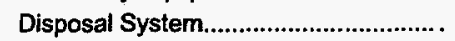 & 213.6 & 217.5 & 221.9 & 233.2 & 101,200 \\
\hline 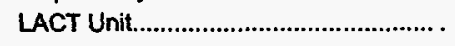 & 174.2 & 171.0 & 171.0 & 171.0 & 15,900 \\
\hline Subtotal or Index ${ }^{* *} \ldots \ldots . . . . . . . . . . . . . . . . . . . . . .$. & 193.4 & 197.7 & 201.9 & 209.8 & 278,800 \\
\hline Total or Index & 175.1 & 190.4 & 197.6 & 210.3 & $1,541,000$ \\
\hline
\end{tabular}

Table D4. Lease Equipment Costs and Indices for Primary Oil Production in Oklahoma (10 Wells Producing from 12,000 Feet by Hydraulic Lift)

\begin{tabular}{|c|c|c|c|c|c|}
\hline \multirow{2}{*}{ Component } & \multicolumn{4}{|c|}{ Index $(1976=100)$} & \multirow{2}{*}{$\begin{array}{c}1997^{*} \\
\text { Cost } \\
\text { (dollars) }\end{array}$} \\
\hline & 1994 & 1995 & 1996 & 1997 & \\
\hline \multicolumn{6}{|l|}{ Producing Equipment: } \\
\hline 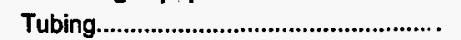 & 136.7 & 144.3 & 149.4 & 163.5 & 826,900 \\
\hline 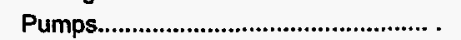 & 250.5 & 261.7 & 280.0 & 280.0 & 209,700 \\
\hline Pumping Equipment................................. & 169.7 & 174.3 & 180.8 & 196.3 & 349,100 \\
\hline 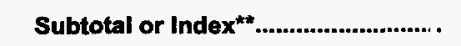 & 155.7 & 162.9 & 169.7 & 182.7 & $1,385,700$ \\
\hline \multicolumn{6}{|l|}{ Gathering System: } \\
\hline 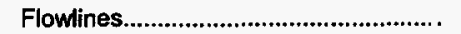 & 193.1 & 197.0 & 202.3 & 203.2 & 150,600 \\
\hline 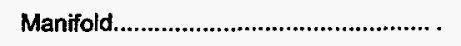 & 264.4 & 268.2 & 274.2 & 290.9 & 38,400 \\
\hline Subtotal or Index ${ }^{\star \star k} \ldots \ldots . . . . . . . . . . . . . . . . . . . .$. & 203.9 & 207.8 & 213.2 & 216.5 & 189,000 \\
\hline \multicolumn{6}{|l|}{ Lease Equipment: } \\
\hline 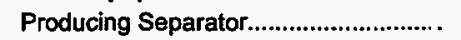 & 173.4 & 173.4 & 182.8 & 187.5 & 12,000 \\
\hline 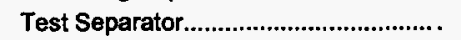 & 189.1 & 188.1 & 187.1 & 198.0 & 20,000 \\
\hline 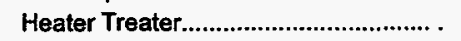 & 136.8 & 145.8 & 150.3 & 154.8 & 24,000 \\
\hline 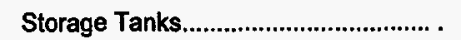 & 195.2 & 200.9 & 205.4 & 215.5 & 72,200 \\
\hline 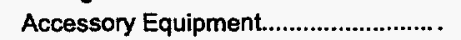 & 212.9 & 220.4 & 227.2 & 227.9 & 33,500 \\
\hline 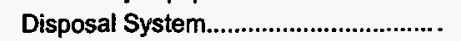 & 187.2 & 190.8 & 194.2 & 201.4 & 72,500 \\
\hline 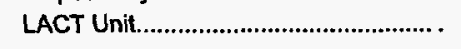 & 174.2 & 171.0 & 171.0 & 171.0 & 15,900 \\
\hline Subtotal or Index $x^{* \ldots} \ldots \ldots . . . . . . . . . . . . . . . . .$. & 184.6 & 188.8 & 192.7 & 199.3 & 250,100 \\
\hline 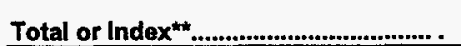 & 163.8 & 170.3 & 176.5 & 187.9 & $1,824,800$ \\
\hline
\end{tabular}


Table D5. Direct Annual Operating Costs and Indices for Primary Oil Production in Oklahoma (10 Wells Producing from 2,000 Feet by Rod Lift)

\begin{tabular}{|c|c|c|c|c|c|}
\hline \multirow{2}{*}{ Component } & \multicolumn{4}{|c|}{ Index $(1976=100)$} & \multirow{2}{*}{$\begin{array}{c}1997^{*} \\
\text { Cost } \\
\text { (dollars) }\end{array}$} \\
\hline & 1994 & 1995 & 1996 & 1997 & \\
\hline \multicolumn{6}{|l|}{ Normal Daily Expense: } \\
\hline 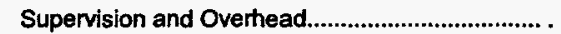 & 300.0 & 320.0 & 331.7 & 346.7 & 20,800 \\
\hline Labor (pumper) & 223.0 & 223.0 & 223.0 & 248.6 & 18,400 \\
\hline 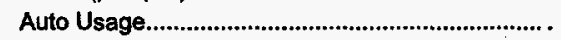 & 272.0 & 284.0 & 296.0 & 308.0 & 7,700 \\
\hline Chemicals & 207.4 & 203.7 & 203.7 & 218.5 & 5,900 \\
\hline Fuel, Power \& Water.............................................. . & 492.6 & 451.9 & 585.2 & 613.0 & 33,100 \\
\hline 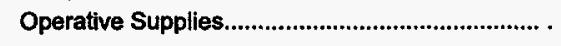 & 233.3 & 233.3 & 233.3 & 250.0 & 1,500 \\
\hline Subtotal or Index & 304.5 & 301.2 & 334.6 & 355.3 & 87,400 \\
\hline \multicolumn{6}{|l|}{ Surface Maintenance, Repair \& Services: } \\
\hline 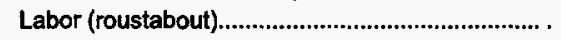 & 220.7 & 220.7 & 220.7 & 244.8 & 7,100 \\
\hline Supplies \& Services & 234.4 & 234.4 & 234.4 & 259.4 & 8,300 \\
\hline 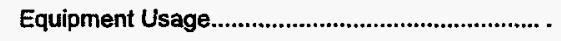 & 284.6 & 292.3 & 284.6 & 346.2 & 4,500 \\
\hline 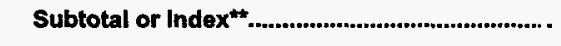 & 237.8 & 239.2 & 237.8 & 268.9 & 19,900 \\
\hline \multicolumn{6}{|l|}{ Subsurface Maintenance, Repair \& Services: } \\
\hline 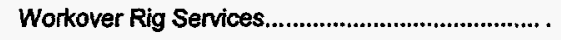 & 140.3 & 140.3 & 145.2 & 153.2 & 9,500 \\
\hline 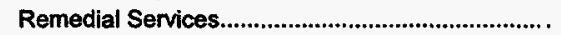 & 150.0 & 156.3 & 156.3 & 162.5 & 2,600 \\
\hline 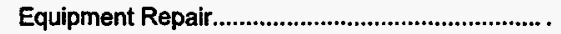 & 140.0 & 146.7 & 151.1 & 160.0 & 7,200 \\
\hline Other & 200.0 & 200.0 & 200.0 & 200.0 & 400 \\
\hline Subtotal or Index $* *$ & 142.4 & 145.6 & 149.6 & 157.6 & 19,700 \\
\hline Total or Index ${ }^{\star k}$ & 247.9 & 247.2 & 266.5 & 285.4 & 127,000 \\
\hline
\end{tabular}

Table D6. Direct Annual Operating Costs and Indices for Primary Oil Production in Oklahoma (10. Wells Producing from 4,000 Feet by Rod Lift)

\begin{tabular}{|c|c|c|c|c|c|}
\hline \multirow{2}{*}{ Component } & \multicolumn{4}{|c|}{ Index $(1976=100)$} & \multirow{2}{*}{$\begin{array}{c}1997^{*} \\
\text { Cost } \\
\text { (dollars) }\end{array}$} \\
\hline & 1994 & 1995 & 1996 & 1997 & \\
\hline \multicolumn{6}{|l|}{ Normal Dally Expense: } \\
\hline 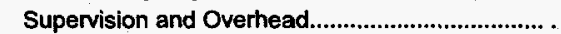 & 297.1 & 317.6 & 330.9 & 345.6 & 23,500 \\
\hline Labor (pumper) & 223.0 & 223.0 & 223.0 & 248.6 & 18,400 \\
\hline 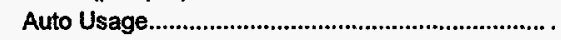 & 272.0 & 284.0 & 296.0 & 308.0 & 7,700 \\
\hline 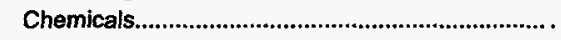 & 248.1 & 244.4 & 244.4 & 263.0 & 7,100 \\
\hline 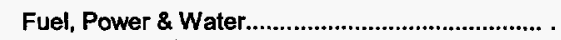 & 465.2 & 422.7 & 559.1 & 589.4 & 38,900 \\
\hline 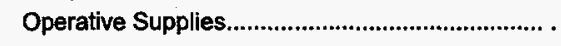 & 233.3 & 233.3 & 233.3 & 266.7 & 1,600 \\
\hline Subtotal or Index & 309.4 & 304.9 & 343.2 & 365.4 & 97,200 \\
\hline \multicolumn{6}{|l|}{ Surface Maintenance, Repair \& Services: } \\
\hline 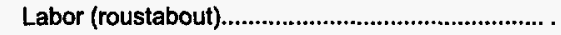 & 220.7 & 220.7 & 220.7 & 244.8 & 7,100 \\
\hline 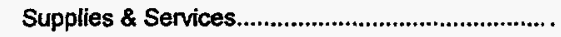 & 235.3 & 238.2 & 238.2 & 261.8 & 8,900 \\
\hline 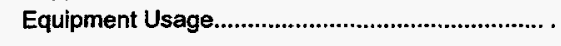 & 300.0 & 300.0 & 300.0 & 361.5 & 4,700 \\
\hline Subtotal or Index ${ }^{* *}$ & 240.8 & 242.1 & 242.1 & 272.4 & 20,700 \\
\hline \multicolumn{6}{|l|}{ Subsurface Maintenance, Repair \& Services: } \\
\hline 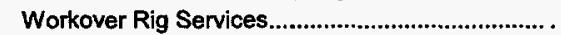 & 161.7 & 161.7 & 167.0 & 175.5 & 16,500 \\
\hline 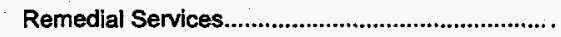 & 158.3 & 162.5 & 170.8 & 175.0 & 4,200 \\
\hline 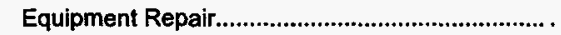 & 126.0 & 146.0 & 158.0 & 168.0 & 8,400 \\
\hline 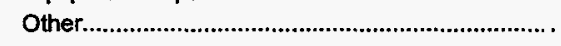 & 250.0 & 250.0 & 250.0 & 250.0 & 500 \\
\hline Subtotal or Index & 151.8 & 158.2 & 165.9 & $\mathbf{1 7 4 . 1}$ & 29,600 \\
\hline Total or Index $x^{* *} \ldots$ & 246.9 & 246.9 & 269.3 & 288.1 & 147,500 \\
\hline
\end{tabular}


Table D7. Direct Annual Operating Costs and Indices for Primary Oll Production in Oklahoma (10 Wells Producing from 8,000 Feet by Hydraulic Lift)

\begin{tabular}{|c|c|c|c|c|c|}
\hline \multirow{2}{*}{ Component } & \multicolumn{4}{|c|}{ Index $(1976=100)$} & \multirow{2}{*}{$\begin{array}{c}1997^{*} \\
\text { Cost } \\
\text { (dolllars) }\end{array}$} \\
\hline & 1994 & 1995 & 1996 & 1997 & \\
\hline \multicolumn{6}{|l|}{ Normal Dally Expense: } \\
\hline 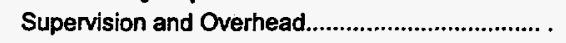 & 298.7 & 320.3 & 332.9 & 348.1 & 27,500 \\
\hline Labor (pumper) & 223.0 & 223.0 & 223.0 & 248.6 & 113,400 \\
\hline 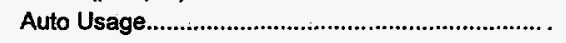 & 272.0 & 284.0 & 296.0 & 308.0 & 3,700 \\
\hline 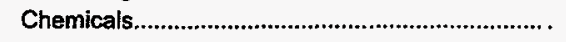 & $\uparrow 79.3$ & 179.3 & 179.3 & 193.1 & 5,600 \\
\hline 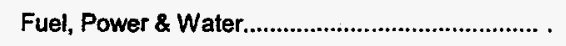 & 425.5 & 380.9 & 522.3 & 553.2 & 52,000 \\
\hline Operative Supplies.................................................. . & 300.0 & 300.0 & 300.0 & 312.5 & 2,500 \\
\hline Subtotal or Index & 305.8 & 298.7 & $\mathbf{3 4 6 . 0}$ & 368.0 & 113,700 \\
\hline \multicolumn{6}{|l|}{ Subsurface Maintenance, Repair \& Services: } \\
\hline 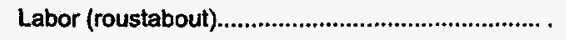 & 220.7 & 220.7 & 220.7 & 244.8 & $7^{\prime}, 100$ \\
\hline 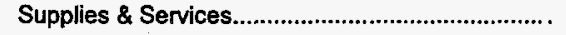 & 222.1 & 223.4 & 224.7 & 237.7 & 18,300 \\
\hline 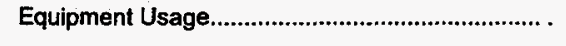 & 286.7 & 293.3 & 286.7 & 346.7 & 5,200 \\
\hline Subtotal or Index & 229.8 & 231.4 & 231.4 & 252.9 & 30,600 \\
\hline \multicolumn{6}{|l|}{ Subsurface Maintenance, } \\
\hline 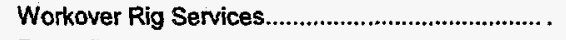 & 183.6 & 183.6 & 185.2 & 191.8 & 11,700 \\
\hline Remedial Services.................................................... & 179.7 & 182.3 & 191.1 & 200.0 & 15,800 \\
\hline Equipment Repair................................................................ & 404.5 & 423.6 & 453.4 & 453.4 & 80,700 \\
\hline 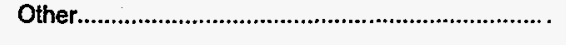 & 214.3 & 228.6 & 228.6 & 228.6 & 1,600 \\
\hline Subtotal or Index & 304.3 & 315.7 & 334.5 & 337.8 & 109,800 \\
\hline Total or Index ${ }^{* *}$ & 293.0 & 295.2 & 322.6 & 336.6 & 254,100 \\
\hline \multirow{2}{*}{ Component } & \multicolumn{4}{|c|}{ Index $(1976=100)$} & $1997^{*}$ \\
\hline & 1994 & 1995 & 1996 & 1997 & (dollars) \\
\hline \multicolumn{6}{|l|}{ Normal Daily Expense: } \\
\hline 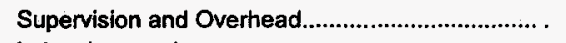 & 298.0 & 320.4 & 333.7 & 349.0 & 34,200 \\
\hline Labor (pumper) & 223.0 & 223.0 & 223.0 & 248.6 & 18,400 \\
\hline Auto Usage & 272.0 & 284.0 & 296.0 & 308.0 & 7,700 \\
\hline 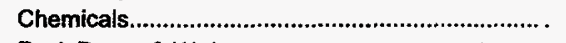 & 193.9 & 190.9 & 190.9 & 206.1 & 6,800 \\
\hline 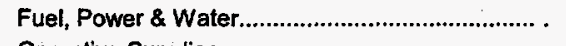 & 403.1 & 356.7 & 500.8 & 533.1 & 67,700 \\
\hline Operative Supplies.............................................. & 333.3 & 333.3 & 333.3 & 355.6 & 3,200 \\
\hline Subtotal or Index & 309.0 & 299.5 & 353.8 & 377.0 & 138,000 \\
\hline \multicolumn{6}{|l|}{ Surface Maintenance, Repair \& Services: } \\
\hline 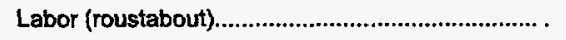 & 220.7 & 220.7 & 220.7 & 244.8 & 7,100 \\
\hline 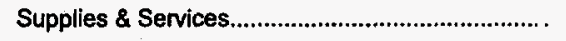 & 232.0 & 233.0 & 233.0 & 245.6 & 25,300 \\
\hline 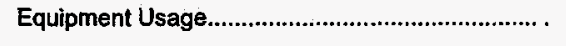 & 286.7 & 293.3 & 286.7 & 346.7 & 5,200 \\
\hline Subtotal or Index & 235.4 & 236.7 & 236.1 & 255.8 & 37,600 \\
\hline \multicolumn{6}{|l|}{ Subsurface Maintenance, Repair \& Services: } \\
\hline 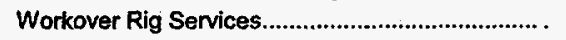 & 186.8 & 186.8 & 190.1 & 195.6 & 17,800 \\
\hline 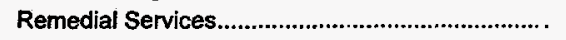 & 241.7 & 244.7 & 260.2 & 268.9 & 27,700 \\
\hline 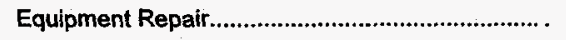 & 403.4 & 421.8 & 450.8 & 451.4 & 80,800 \\
\hline 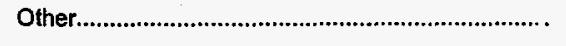 & 233.3 & 233.3 & 233.3 & 244.4 & 2,200 \\
\hline Subtotal or Index & 304.2 & 313.6 & 332.2 & 336.4 & 128,500 \\
\hline Total or Index ${ }^{ \pm \pm} \ldots$ & 294.9 & 295.2 & 325.3 & 339.8 & 304,100 \\
\hline
\end{tabular}


Table E1. Lease Equipment Costs and Indices for Primary Oil Production in the Rocky Mountains (10 Wells Producing from 2,000 Feet by Rod Lift)

\begin{tabular}{|c|c|c|c|c|c|}
\hline \multirow{2}{*}{ Component } & \multicolumn{4}{|c|}{ Index $(1976=100)$} & \multirow{2}{*}{$\begin{array}{c}1997^{*} \\
\text { Cost } \\
\text { (dollars) }\end{array}$} \\
\hline & 1994 & 1995 & 1996 & 1997. & \\
\hline \multicolumn{6}{|l|}{ Producing Equipment: } \\
\hline Tubing & 104.3 & 146.4 & 149.1 & 165.3 & 62,000 \\
\hline 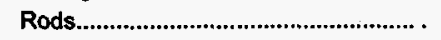 & 90.9 & 91.7 & 92.5 & 94.5 & 24,000 \\
\hline 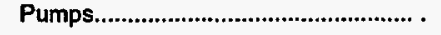 & 147.6 & 152.4 & 157.1 & 166.7 & 14,000 \\
\hline 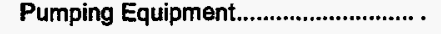 & 160.7 & 172.9 & 190.0 & 247.9 & 243,400 \\
\hline Subtotal or Index & 137.1 & 153.9 & 164.7 & 202.6 & 343,400 \\
\hline \multicolumn{6}{|l|}{ Gathering System: } \\
\hline 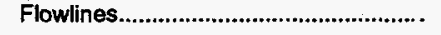 & 261.8 & 265.1 & 271.1 & 294.1 & 44,700 \\
\hline 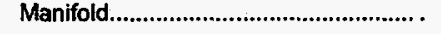 & 261.7 & 264.7 & 271.4 & 288.0 & 38,300 \\
\hline Subtotal or Index & 261.8 & 264.9 & 271.2 & 291.2 & 83,000 \\
\hline \multicolumn{6}{|l|}{ Lease Equipment: } \\
\hline 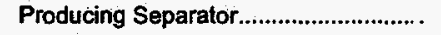 & 173.4 & 173.4 & 182.8 & 187.5 & 12,000 \\
\hline 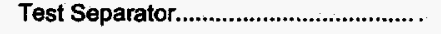 & 189.1 & 188.1 & 187.1 & 198.0 & 20,000 \\
\hline 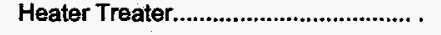 & 102.3 & 108.8 & 112.0 & 116.6 & 25,300 \\
\hline 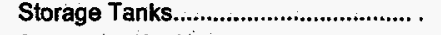 & 189.9 & 194.9 & 199.4 & 208.1 & 69,700 \\
\hline 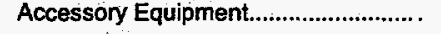 & 213.6 & 221.1 & 227.9 & 229.3 & 33,700 \\
\hline Disposal System & 219.1 & 224.0 & 228.3 & 234.4 & 81,100 \\
\hline 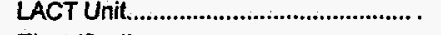 & 174.2 & 171.0 & 171.0 & 171.0 & 15,900 \\
\hline 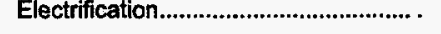 & 244.4 & 247.3 & 250.6 & 254.8 & 61,400 \\
\hline 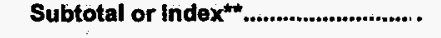 & 193.2 & 197.2 & 201.1 & 206.7 & 319,100 \\
\hline Total or Index $x^{+4}$ & 171.8 & 181.8 & 189.3 & 211.5 & 745,500 \\
\hline \multirow{2}{*}{ Component } & \multicolumn{4}{|c|}{ Index $(1976=100)$} & $\begin{array}{l}1997^{*} \\
\text { Cost }\end{array}$ \\
\hline & 1994 & 1995 & 1996 & 1997 & (dollars) \\
\hline \multicolumn{6}{|l|}{ Producing Equipment: } \\
\hline 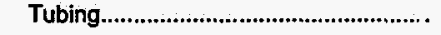 & 101.8 & 143.6 & 146.2 & 162.2 & 123,300 \\
\hline 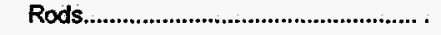 & 98.0 & 98.7 & 99.3 & 100.4 & 45,100 \\
\hline Pumps. & 134.1 & 154.9 & 174.4 & 185.4 & 15,200 \\
\hline 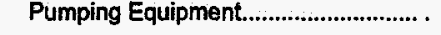 & 137.9 & 159.5 & 172.2 & 200.2 & 372,100 \\
\hline Subtotal or Index & 123.4 & 146.9 & 155.6 & 176.4 & 555,700 \\
\hline \multicolumn{6}{|l|}{ Gathering System: } \\
\hline 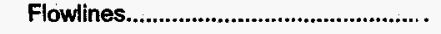 & 250.5 & 253.5 & 258.6 & 281.3 & 55,700 \\
\hline 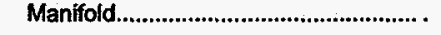 & 261.7 & 264.7 & 271.4 & 288.0 & 38,300 \\
\hline Subtotal or Index & 255.0 & 258.0 & 263.7 & 284.0 & 94,000 \\
\hline \multicolumn{6}{|l|}{ Lease Equipment: } \\
\hline 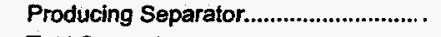 & 173.4 & 173.4 & 182.8 & 187.5 & 12,000 \\
\hline 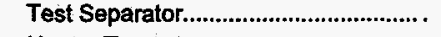 & 189.1 & 188.1 & 187.1 & 198.0 & 20,000 \\
\hline 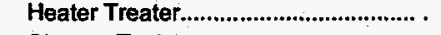 & 102.3 & 108.8 & 112.0 & 116.6 & 25,300 \\
\hline 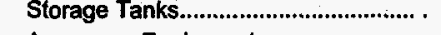 & 189.9 & 194.9 & 199.4 & 208.1 & 69,700 \\
\hline 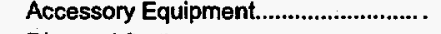 & 213.6 & 221.1 & 227.9 & 229.3 & 33,700 \\
\hline Disposal System & 221.9 & 226.8 & 230.8 & 237.3 & 83,300 \\
\hline 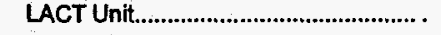 & 174.2 & 171.0 & 171.0 & 171.0 & 15,900 \\
\hline 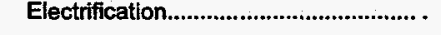 & 242.5 & 245.6 & 249.8 & 253.0 & 72,600 \\
\hline Subtotal or Index ${ }^{* *}$............................... & 195.0 & 199.1 & 203.0 & 208.5 & 332,500 \\
\hline Total or Index & 154.5 & 170.5 & 177.6 & 193.5 & 982,200 \\
\hline
\end{tabular}


Table E3. Lease Equipment Costs and Indices for Primary OII Production in the Rocky Mountains (10 Wells Producing from 8,000 Feet by Rod Lift)

\begin{tabular}{|c|c|c|c|c|c|}
\hline \multirow{2}{*}{ Component } & \multicolumn{4}{|c|}{ Index $(1976=100)$} & \multirow{2}{*}{$\begin{array}{c}1997^{*} \\
\text { Cost } \\
\text { (dollars) }\end{array}$} \\
\hline & 1994 & 1995 & 1996 & 1997 & \\
\hline \multicolumn{6}{|l|}{ Producing Equipment: } \\
\hline 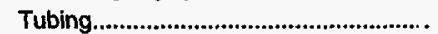 & 105.6 & 139.9 & 142.8 & 158.5 & 311,200 \\
\hline 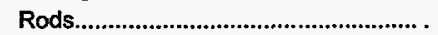 & 100.5 & 100.8 & 101.1 & 101.7 & 111,300 \\
\hline 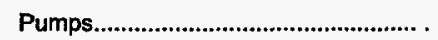 & 157.8 & 172.2 & 187.8 & 197.8 & 17,800 \\
\hline 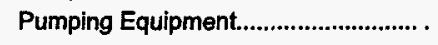 & 142.0 & 155.4 & 168.1 & 195.0 & 721,500 \\
\hline Subtotal or Index $x^{* *}$ & 125.1 & 142.5 & 150.4 & 169.7 & $1,161,800$ \\
\hline \multicolumn{6}{|l|}{ Gathering System: } \\
\hline 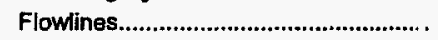 & 240.7 & 243.2 & 247.3 & 270.7 & 73,900 \\
\hline 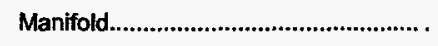 & 261.7 & 264.7 & 271.4 & 288.0 & 38,300 \\
\hline 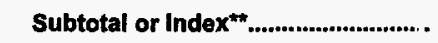 & 247.5 & 250.2 & 255.2 & 276.4 & 112,200 \\
\hline \multicolumn{6}{|l|}{ Lease Equipment: } \\
\hline 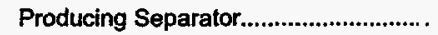 & 173.4 & 173.4 & 182.8 & 187.5 & 12,0100 \\
\hline Test Separator............................................. & 189.1 & 188.1 & 187.1 & 198.0 & 20,000 \\
\hline 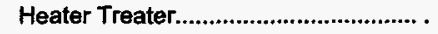 & 102.3 & 108.8 & 112.0 & 116.6 & 25,300 \\
\hline 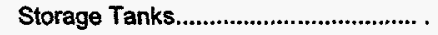 & 189.9 & 194.9 & 199.4 & 208.1 & 69,700 \\
\hline Accessory Equipment................................... & 213.6 & 221.1 & 227.9 & 229.3 & 33,700 \\
\hline 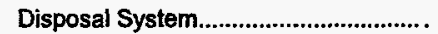 & 220.4 & 224.7 & 228.6 & 235.5 & 88,800 \\
\hline 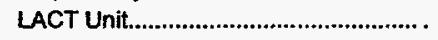 & 174.2 & 171.0 & 171.0 & 171.0 & 15,900 \\
\hline 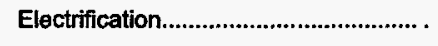 & 245.7 & 248.0 & 254.0 & 254.3 & 99,700 \\
\hline Subtotal or Index ${ }^{* *}$ & 198.7 & 202.4 & 206.1 & 211.5 & 365,100 \\
\hline Total or Index $x^{*+\ldots}$ & 144.8 & 158.9 & 165.9 & 182.5 & $1,639,100$ \\
\hline
\end{tabular}

Table E4. Lease Equipment Costs and Indices for Primary Oll Production in the Rocky Mountains (10 Wells Producing from 12,000 Feet by Hydraulic Lift)

\begin{tabular}{|c|c|c|c|c|c|}
\hline \multirow{2}{*}{ Component } & \multicolumn{4}{|c|}{ Index $(1976=100)$} & \multirow{2}{*}{$\begin{array}{c}1997^{\prime \prime} \\
\text { Cost } \\
\text { (dollars) }\end{array}$} \\
\hline & 1994 & 1995 & 1996 & 1997 & \\
\hline \multicolumn{6}{|l|}{ Producing Equipment: } \\
\hline 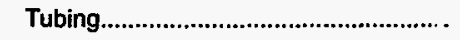 & 131.1 & 138.4 & 143.2 & 156.7 & 829,300 \\
\hline 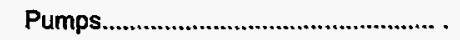 & 250.5 & 261.7 & 280.0 & 280.0 & 209,700 \\
\hline Pumping Equipment................................ & 205.3 & 213.0 & 223.2 & 226.3 & 312,300 \\
\hline Subtotal or Index $x^{* *}$ & 157.0 & 164.7 & 171.9 & 182.1 & $1,351,300$ \\
\hline \multicolumn{6}{|l|}{ Gathering System: } \\
\hline 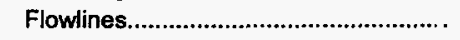 & 189.7 & 192.6 & 197.1 & 205.2 & 161,100 \\
\hline 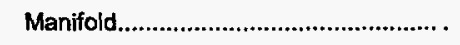 & 261.7 & 264.7 & 271.4 & 288.0 & 38,300 \\
\hline Subtotal or Index ${ }^{* *} \ldots$ & 200.1 & 203.1 & 207.8 & 217.2 & 199,400 \\
\hline \multicolumn{6}{|l|}{ Lease Equipment: } \\
\hline 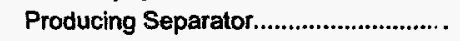 & 173.4 & 173.4 & 182.8 & 187.5 & 12,000 \\
\hline 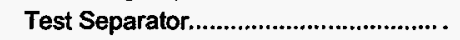 & 189.1 & 188.1 & 187.1 & 198.0 & 20,000 \\
\hline 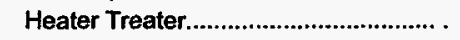 & 102.3 & 108.8 & 112.0 & 116.6 & 25,300 \\
\hline 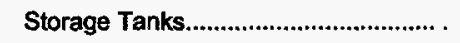 & 189.9 & 194.9 & 199.4 & 208.1 & 69,700 \\
\hline 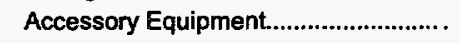 & 213.6 & 221.1 & 227.9 & 229.3 & 33,700 \\
\hline Disposal System & 223.3 & 228.1 & 231.6 & 238.5 & 89,900 \\
\hline 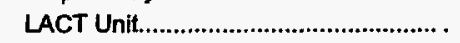 & 174.2 & 171.0 & 171.0 & 171.0 & 15,900 \\
\hline 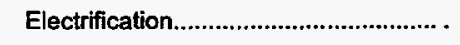 & 262.0 & 262.7 & 264.6 & 269.0 & 42,500 \\
\hline 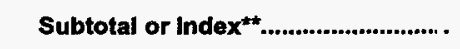 & 193.8 & 197.7 & 201.2 & 207.1 & 309,000 \\
\hline 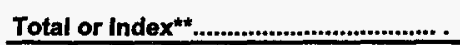 & 166.6 & 173.3 & 179.7 & 189.1 & $1,859,700$ \\
\hline
\end{tabular}


Table E5. Direct Annual Operating Costs and Indices for Primary Oll Production in the Rocky Mountains (10 Wells Producing from 2,000 Feet by Rod Lift)

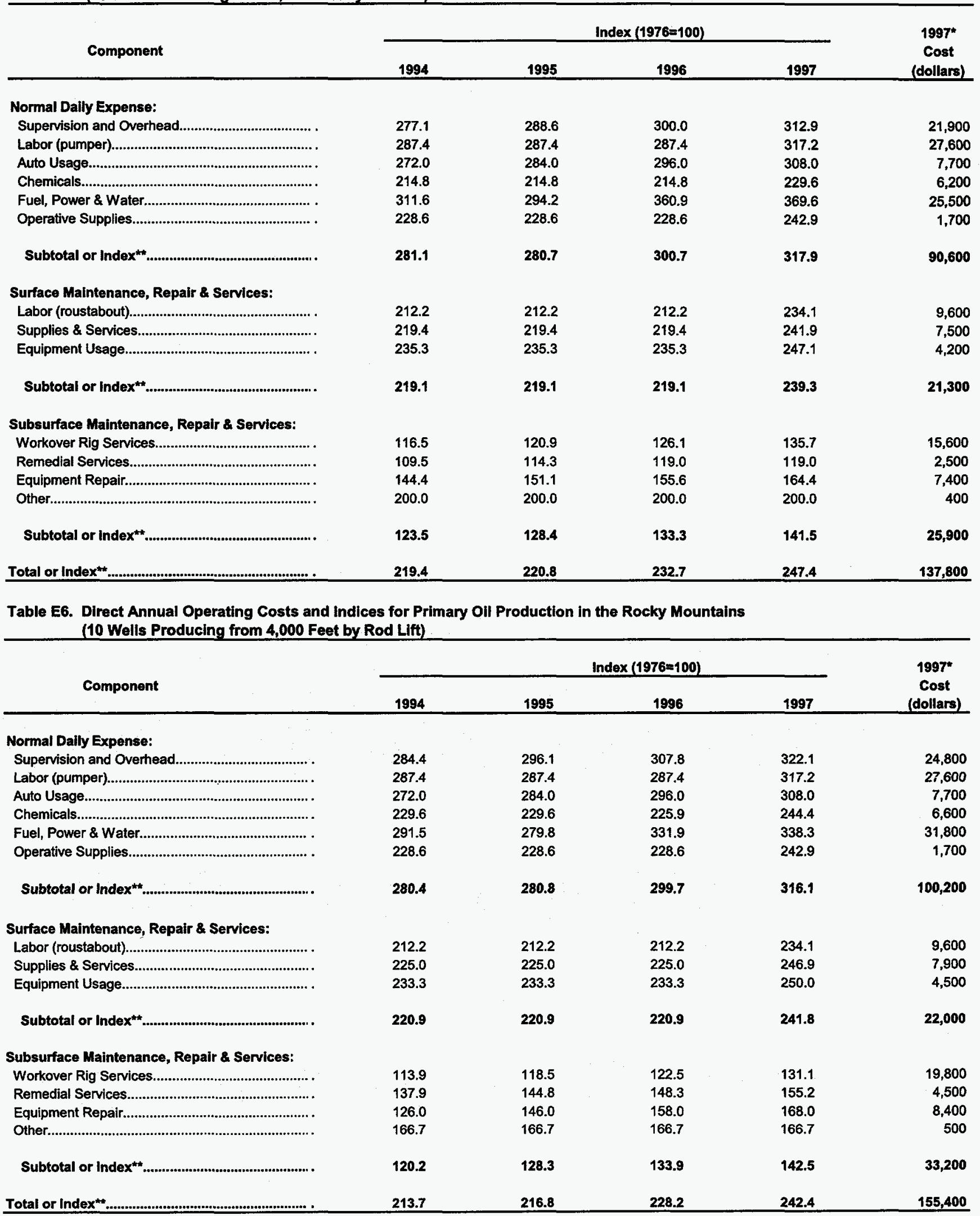


Table E7. Direct Annual Operating Costs and Indices for Primary Oil Production in the Rocky Mountains (10 Wells Producing from 8,000 Feet by Rod Lift)

\begin{tabular}{|c|c|c|c|c|c|}
\hline \multirow{2}{*}{ Component } & \multicolumn{4}{|c|}{ Index $(1976=100)$} & \multirow{2}{*}{$\begin{array}{c}19917^{*} \\
\text { Cost } \\
\text { (dollars) }\end{array}$} \\
\hline & 1994 & 1995 & 1996 & 1997 & \\
\hline \multicolumn{6}{|l|}{ Normal Dally Expense: } \\
\hline 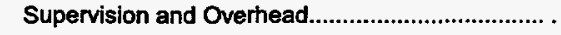 & 284.4 & 296.7 & 308.9 & 323.3 & 29,100 \\
\hline 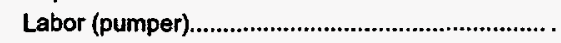 & 287.4 & 287.4 & 287.4 & 317.2 & 27,600 \\
\hline Auto Usage & 272.0 & 284.0 & 296.0 & 308.0 & 7,700 \\
\hline 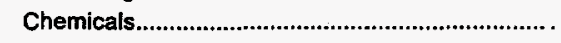 & 210.3 & 206.9 & 206.9 & 220.7 & $6 i, 400$ \\
\hline Fuel, Power \& Water........................................... . & 282.1 & 274.5 & 313.1 & 317.2 & 46,000 \\
\hline 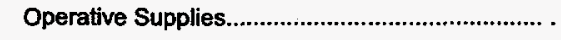 & 228.6 & 228.6 & 228.6 & 257.1 & 1,800 \\
\hline Subtotal or Index & 276.8 & 277.3 & 295.6 & 309.7 & 118,600 \\
\hline \multicolumn{6}{|l|}{ Surface Maintenance, Repair \& Services: } \\
\hline 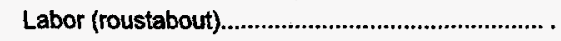 & 212.2 & 212.2 & 212.2 & 234.1 & 9,600 \\
\hline 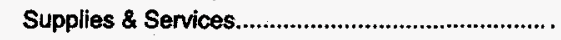 & 224.2 & 224.2 & 224.2 & 245.5 & 8,100 \\
\hline 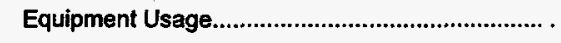 & 242.1 & 242.1 & 242.1 & 263.2 & 5,000 \\
\hline Subtotal or Index ${ }^{* \hbar}$ & 222.6 & 222.6 & 222.6 & 244.1 & 22,700 \\
\hline \multicolumn{6}{|l|}{ Subsurface Maintenance, Repair \& Services: } \\
\hline 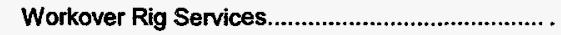 & 120.5 & 124.4 & 128.3 & 136.8 & 35,300 \\
\hline 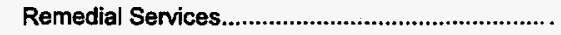 & 205.0 & 215.0 & 227.5 & 238.8 & 19,100 \\
\hline 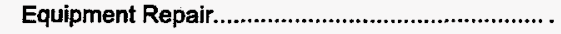 & 163.3 & 183.3 & 191.7 & 201.7 & 12,100 \\
\hline Other & 150.0 & 160.0 & 160.0 & 160.0 & 1,600 \\
\hline Subtotal or Index & 144.1 & 151.7 & 157.8 & 166.9 & 63,100 \\
\hline 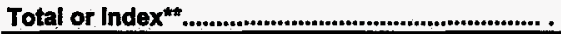 & 209.8 & 213.6 & 224.3 & 236.9 & 209,400 \\
\hline
\end{tabular}

Table E8. Direct Annual Operating Costs and Indices for Primary Oil Production in the Rocky Mountains (10 Wells Producing from 12,000 Feet by Hydraulic Lift)

\begin{tabular}{|c|c|c|c|c|c|}
\hline \multirow{2}{*}{ Component } & \multicolumn{4}{|c|}{ Index $(1976=100)$} & \multirow{2}{*}{$\begin{array}{c}1997^{*} \\
\text { Coskt } \\
\text { (dollars) }\end{array}$} \\
\hline & 1994 & 1995 & 1996 & 1997 & \\
\hline \multicolumn{6}{|l|}{ Normal Daily Expense: } \\
\hline Supervision and Overhead..................................... & 283.9 & 297.3 & 308.9 & 324.1 & 36,300 \\
\hline 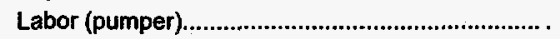 & 287.4 & 287.4 & 287.4 & 317.2 & 27,600 \\
\hline 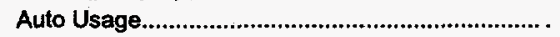 & 272.0 & 284.0 & 296.0 & 308.0 & 7,700 \\
\hline 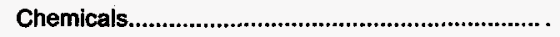 & 200.0 & 197.0 & 197.0 & 212.1 & 7,000 \\
\hline Fuel, Power \& Water................................................ . & 299.5 & 294.0 & 322.1 & 325.1 & 64,700 \\
\hline Operative Supplies........................................................ . & 230.0 & 230.0 & 230.0 & 240.0 & 2,400 \\
\hline Subtotal or Index & 283.5 & 284.8 & 300.2 & 312.7 & 145,700 \\
\hline \multicolumn{6}{|l|}{ Surface Maintenance, Repair \& Services: } \\
\hline Labor (roustabout) & 212.2 & 212.2 & 212.2 & 234.1 & 9,600 \\
\hline Supplies \& Services................................................ & 231.7 & 233.3 & 235.0 & 246.7 & 14,800 \\
\hline 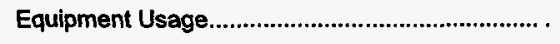 & 242.1 & 242.1 & 242.1 & 263.2 & 5,000 \\
\hline 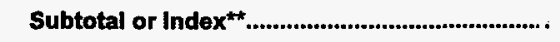 & 226.7 & 227.5 & 228.3 & 245.0 & 29,400 \\
\hline \multicolumn{6}{|l|}{ Subsurface Maintenance, Repair \& Services: } \\
\hline Workover Rig Services.............................................. & 133.3 & 135.3 & 138.2 & 144.1 & 14,700 \\
\hline 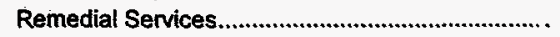 & 167.7 & 178.0 & 188.2 & 196.9 & 25.000 \\
\hline 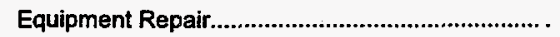 & 403.9 & 421.8 & 450.8 & 451.4 & 80,800 \\
\hline 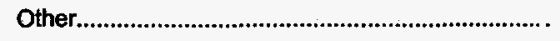 & 175.0 & 175.0 & 175.0 & 183.3 & 2,200 \\
\hline Subtotal or Index & 260.2 & 271.4 & 287.6 & 292.1 & 122,700 \\
\hline Total or Index & 267.0 & 272.4 & 286.4 & 296.0 & 297,800 \\
\hline
\end{tabular}


Table F1. Lease Equipment Costs and Indices for Primary Oil Production in California (10 Wells Producing from 2,000 Feet by Rod Lift)

\begin{tabular}{|c|c|c|c|c|c|}
\hline \multirow{2}{*}{ Component } & \multicolumn{4}{|c|}{ Index $(1976=100)$} & \multirow{2}{*}{$\begin{array}{c}1997^{*} \\
\text { Cost } \\
\text { (dollars) }\end{array}$} \\
\hline & 1994 & 1995 & 1996 & 1997 & \\
\hline \multicolumn{6}{|l|}{ Producing Equipment: } \\
\hline 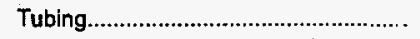 & 107.9 & 143.0 & 145.9 & 162.0 & 77,900 \\
\hline 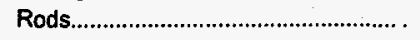 & 110.8 & 111.2 & 111.5 & 112.9 & 32,300 \\
\hline 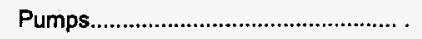 & 153.8 & 147.9 & 142.0 & 150.4 & 17,900 \\
\hline Pumping Equipment............................... & 127.6 & 146.5 & 158.1 & 187.9 & 316,300 \\
\hline Subtotal or Index $x^{* *} . . . . . . . . . . . . . . . . . . . . . .$. & 123.3 & 142.0 & 149.9 & 173.0 & 444,400 \\
\hline \multicolumn{6}{|l|}{ Gathering System: } \\
\hline 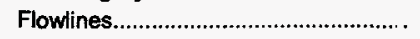 & 242.3 & 224.9 & 247.7 & 250.2 & 70,300 \\
\hline Manifold................................................. & 261.9 & 264.9 & 271.6 & 288.1 & 38,600 \\
\hline 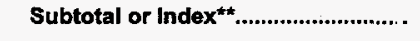 & 248.7 & 237.8 & 255.4 & 262.4 & 108,900 \\
\hline \multicolumn{6}{|l|}{ Lease Equipment: } \\
\hline Producing Separator.............................. & 165.7 & 165.7 & 174.6 & 179.1 & 12,000 \\
\hline 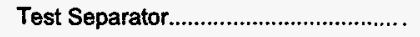 & 189.1 & 188.1 & 187.1 & 198.0 & 20,000 \\
\hline Free water knockout & 133.3 & 134.7 & 141.3 & 146.7 & 11,000 \\
\hline Heater Treater......................................... . & 231.7 & 243.2 & 249.0 & 256.4 & 151,300 \\
\hline Storage Tanks........................................ & 181.0 & 185.9 & 190.2 & 194.8 & 67,600 \\
\hline 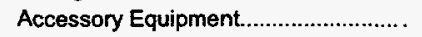 & 212.9 & 220.4 & 227.2 & 227.9 & 33,500 \\
\hline 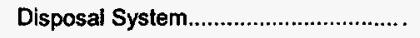 & 183.6 & 187.0 & 193.5 & 202.3 & 71,400 \\
\hline 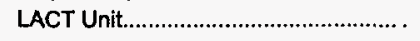 & 174.2 & 171.0 & 171.0 & 171.0 & 15,900 \\
\hline Electrification..................................... & 247.3 & 250.8 & 255.3 & 258.0 & 67,600 \\
\hline Subtotal or Index ${ }^{* *} \ldots$ & 204.8 & 210.4 & 215.5 & 221.3 & 450,300 \\
\hline Total or Index & 166.7 & 177.7 & 185.2 & 200.0 & $1,003,600$ \\
\hline
\end{tabular}

Table F2. Lease Equipment Costs and Indices for Primary Oil Production in California (10 Wells Producing from 4,000 Feet by Rod Lift)

\begin{tabular}{|c|c|c|c|c|c|}
\hline \multirow{2}{*}{ Component } & \multicolumn{4}{|c|}{ Index $(1976=100)$} & \multirow{2}{*}{$\begin{array}{c}1997^{*} \\
\text { Cost } \\
\text { (dollars) }\end{array}$} \\
\hline & 1994 & 1995 & 1996 & 1997 & \\
\hline \multicolumn{6}{|l|}{ Producing Equipment: } \\
\hline 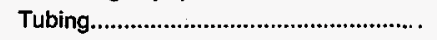 & 105.5 & 140.0 & 142.9 & 158.6 & 155,000 \\
\hline Rods & 105.6 & 105.8 & 106.1 & 107.0 & 59,500 \\
\hline 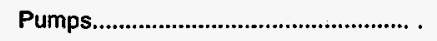 & 153.1 & 146.2 & 140.0 & 146.9 & 19,100 \\
\hline 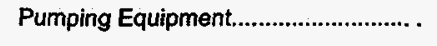 & 108.9 & 124.4 & 134.3 & 155.3 & 422,800 \\
\hline Subtotal or Index ${ }^{* \star}$ & 109.1 & 126.1 & 132.8 & 149.7 & 656,400 \\
\hline \multicolumn{6}{|l|}{ Gathering System: } \\
\hline 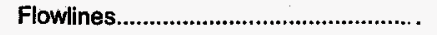 & 228.9 & 210.8 & 233.2 & 235.1 & 91,200 \\
\hline 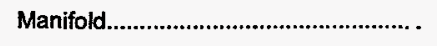 & 261.9 & 264.9 & 271.6 & 288.1 & 38,600 \\
\hline 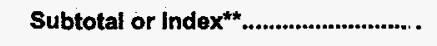 & 237.4 & 224.7 & 243.1 & 248.7 & 129,800 \\
\hline \multicolumn{6}{|l|}{ Lease Equipment: } \\
\hline Producing Separator.................................... & 165.7 & 165.7 & 174.6 & 179.1 & 12,000 \\
\hline 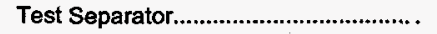 & 189.1 & 188.1 & 187.1 & 198.0 & 20,000 \\
\hline Free water knockout.................................. & 133.3 & 134.7 & 141.3 & 146.7 & 11,000 \\
\hline 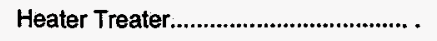 & 231.7 & 243.2 & 249.0 & 256.4 & 151,300 \\
\hline 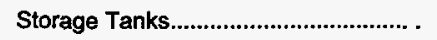 & 181.0 & 185.9 & 190.2 & 194.8 & 67,600 \\
\hline 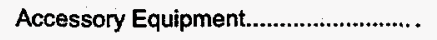 & 212.9 & 220.4 & 227.2 & 227.9 & 33,500 \\
\hline 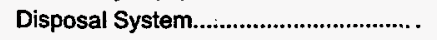 & 180.8 & 183.5 & 190.2 & 198.6 & 73,300 \\
\hline 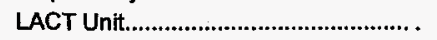 & 174.2 & 171.0 & 171.0 & 171.0 & 15,900 \\
\hline 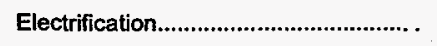 & 225.9 & 229.3 & 232.9 & 235.1 & 77,100 \\
\hline Subtotal or Index $x^{*+\ldots}$ & 202.2 & 207.6 & 212.6 & 218.1 & 461,700 \\
\hline 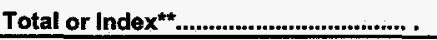 & 146.7 & 158.0 & 165.0 & 177.7 & $1,247,900$ \\
\hline
\end{tabular}


Table F3. Lease Equipment Costs and Indices for Primary Oil Production in California (10 Wells Producing from 8,000 Feet by Hydraulic Lift)

\begin{tabular}{|c|c|c|c|c|c|}
\hline \multirow{2}{*}{ Component } & \multicolumn{4}{|c|}{ Index $(1976=100)$} & \multirow{2}{*}{$\begin{array}{c}1997^{*} \\
\text { Cost } \\
\text { (dollars) }\end{array}$} \\
\hline & 1994 & 1995 & 1996 & 1997 & \\
\hline \multicolumn{6}{|l|}{ Producing Equipment: } \\
\hline 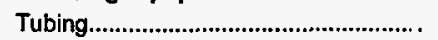 & 107.0 & 112.7 & 114.2 & 129.5 & 506,600 \\
\hline 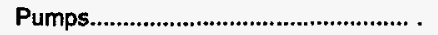 & 250.5 & 261.7 & 280.0 & 280.0 & 209,700 \\
\hline Pumping Equipment................................. . & 196.5 & 203.8 & 213.9 & 215.7 & 297,600 \\
\hline Subtotal or Index & 145.2 & 152.0 & 157.5 & 167.8 & $1,013,900$ \\
\hline \multicolumn{6}{|l|}{ Gathering System: } \\
\hline 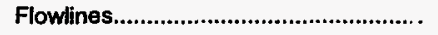 & 206.7 & 209.2 & 203.6 & 203.9 & 196,600 \\
\hline 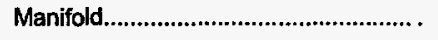 & 261.9 & 264.9 & 271.6 & 288.1 & 38,600 \\
\hline Subtotal or Index & 213.5 & 216.0 & 211.9 & 214.2 & 235,200 \\
\hline \multicolumn{6}{|l|}{ Lease Equipment: } \\
\hline 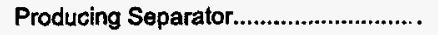 & 165.7 & 165.7 & 174.6 & 179.1 & 12,000 \\
\hline 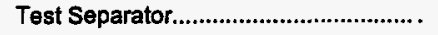 & 189.1 & 188.1 & 187.1 & 198.0 & 20,0100 \\
\hline 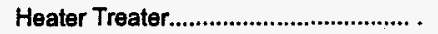 & 133.3 & 134.7 & 141.3 & 146.7 & 11,000 \\
\hline 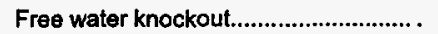 & 231.7 & 243.2 & 249.0 & 256.4 & 151,300 \\
\hline 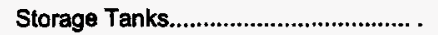 & 181.0 & 185.9 & 190.2 & 194.8 & 67,600 \\
\hline 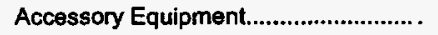 & 212.9 & 220.4 & 227.2 & 227.9 & 33,500 \\
\hline 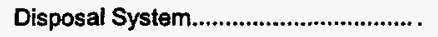 & 180.5 & 182.0 & 189.6 & 197.7 & 78,100 \\
\hline 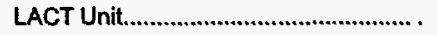 & 174.2 & 171.0 & 171.0 & 171.0 & 15,900 \\
\hline 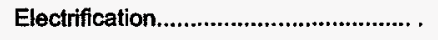 & 250.6 & 251.7 & 253.4 & 256.9 & 44,700 \\
\hline Subtotal or Index & 202.2 & 207.2 & 212.4 & 218.3 & 434,100 \\
\hline Total or Index ${ }^{m+\ldots}$ & 165.9 & 171.7 & 176.0 & 184.4 & $1,683,200$ \\
\hline
\end{tabular}

Table F4. Lease Equipment Costs and Indices for Primary Oil Production in California (10 Wells Producing from 12,000 Feet by Hydraulic Lift)

\begin{tabular}{|c|c|c|c|c|c|}
\hline \multirow{2}{*}{ Component } & \multicolumn{4}{|c|}{ Index $(1976=100)$} & \multirow{2}{*}{$\begin{array}{c}1997^{*} \\
\text { Cost } \\
\text { (dollars) }\end{array}$} \\
\hline & 1994 & 1995 & 1996 & 1997 & \\
\hline \multicolumn{6}{|l|}{ Producing Equipment: } \\
\hline 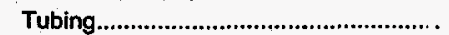 & 118.4 & 125.0 & 129.4 & 141.5 & 828,900 \\
\hline Pumps. & 250.5 & 261.7 & 280.0 & 280.0 & 209,700 \\
\hline Pumping Equipment................................ . & 202.9 & 210.5 & 220.7 & 222.5 & 340,600 \\
\hline 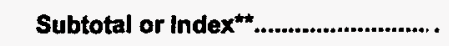 & 146.4 & 153.6 & 160.4 & 169.5 & $1,379,2,00$ \\
\hline \multicolumn{6}{|l|}{ Gathering System: } \\
\hline 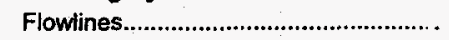 & 206.7 & 209.2 & 203.6 & 203.9 & 196,600 \\
\hline 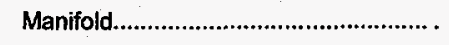 & 261.9 & 264.9 & 271.6 & 288.1 & 38,600 \\
\hline Subtotal or Index ${ }^{* *}$ & 213.5 & 216.0 & 211.9 & 214.2 & 235,200 \\
\hline \multicolumn{6}{|l|}{ Lease Equipment: } \\
\hline 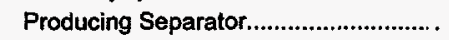 & 165.7 & 165.7 & 174.6 & 179.1 & 12,000 \\
\hline 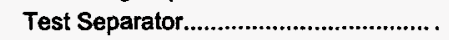 & 189.1 & 188.1 & 187.1 & 198.0 & 20,000 \\
\hline 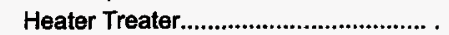 & 133.3 & 134.7 & 141.3 & 146.7 & 11,000 \\
\hline Free water knockout................................. & 231.7 & 243.2 & 249.0 & 256.4 & 151,300 \\
\hline 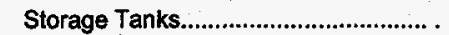 & 181.0 & 185.9 & 190.2 & 194.8 & 67,600 \\
\hline 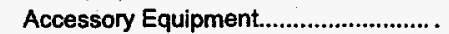 & 212.9 & 220.4 & 227.2 & 227.9 & 33,500 \\
\hline 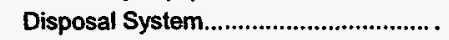 & 180.5 & 182.0 & 189.6 & 197.7 & 78,100 \\
\hline 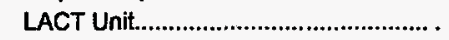 & 174.2 & 171.0 & 171.0 & 171.0 & 15,900 \\
\hline 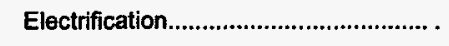 & 258.7 & 258.3 & 258.3 & 265.0 & 54,600 \\
\hline Subtotal or Index ${ }^{* *}$ & 203.8 & 208.6 & 213.5 & 219.7 & 444,000 \\
\hline Total or Index $x^{*+\ldots} \ldots$ & 163.3 & 169.6 & 175.0 & 182.8 & $2,058,400$ \\
\hline
\end{tabular}


Table F5. Direct Annual Operating Costs and Indices for Primary Oll Production in Callfornla (10 Wells Producing from 2,000 Feet by Rod Lift)

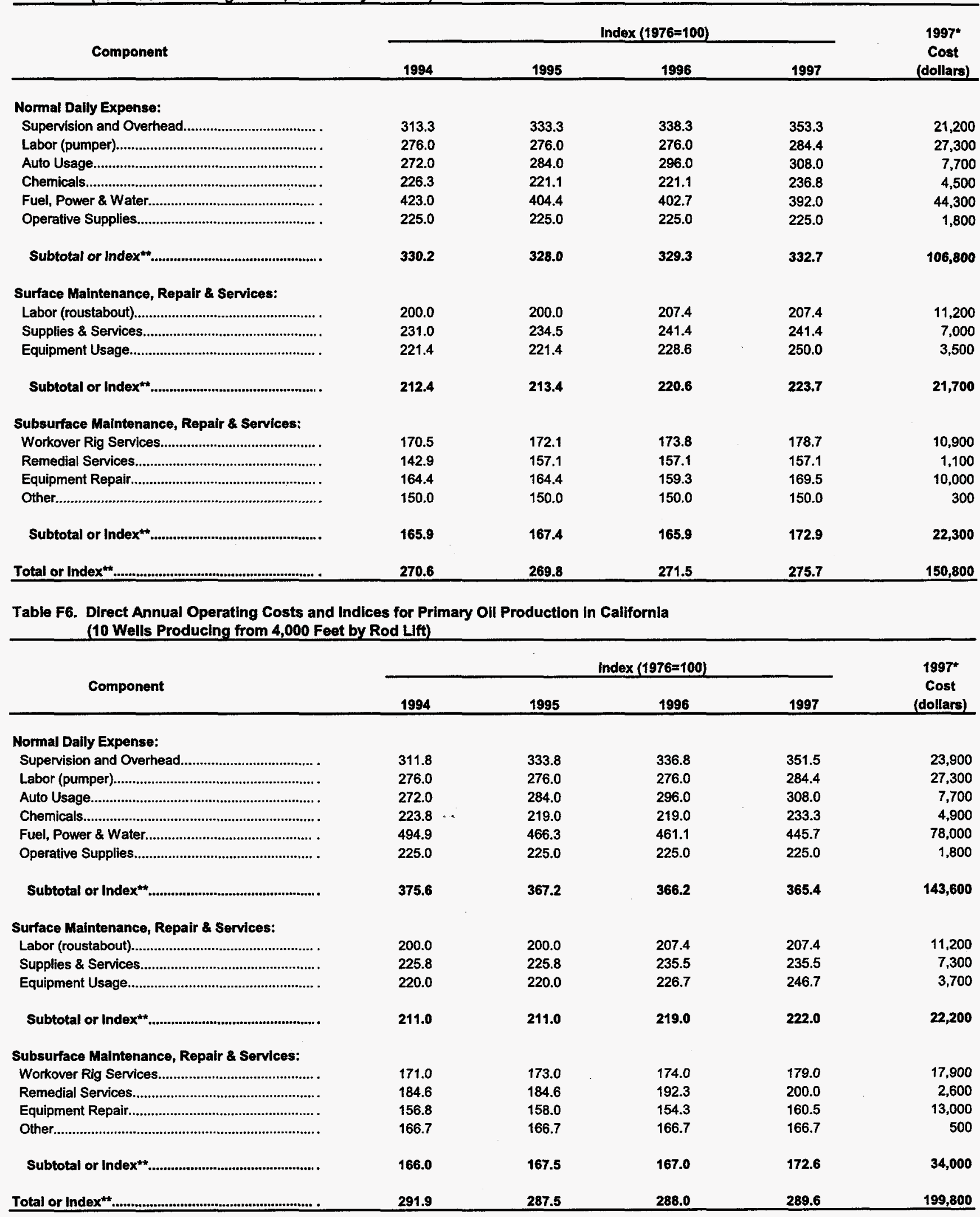


Table F7. Direct Annual Operating Costs and Indices for Primary Oil Production in California (10 Wells Producing from 8,000 Feet by Hydraulic Lift)

\begin{tabular}{|c|c|c|c|c|c|}
\hline \multirow{2}{*}{ Component } & \multicolumn{4}{|c|}{ Index $(1976=100)$} & \multirow{2}{*}{$\begin{array}{c}1997^{*} \\
\text { Cost } \\
\text { (clollars) }\end{array}$} \\
\hline & 1994 & 1995 & 1996 & 1997 & \\
\hline \multicolumn{6}{|l|}{ Normal Daily Expense: } \\
\hline 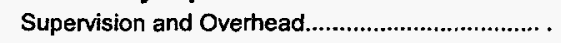 & 312.7 & 335.4 & 339.2 & 354.4 & 28,000 \\
\hline Labor (pumper) & 276.0 & 276.0 & 276.0 & 284.4 & 27,300 \\
\hline 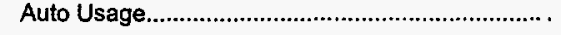 & 272.0 & 284.0 & 296.0 & 308.0 & 7,700 \\
\hline 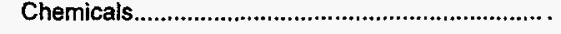 & 220.0 & 220.0 & 216.7 & 230.0 & 6,900 \\
\hline 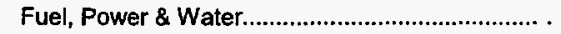 & 551.8 & 515.0 & 503.1 & 482.2 & 173,100 \\
\hline 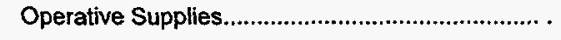 & 200.0 & 200.0 & 209.1 & 209.1 & 2,300 \\
\hline Subtotal or Index & 441.5 & 423.0 & 416.8 & 408.8 & 245,300 \\
\hline \multicolumn{6}{|l|}{ Surface Maintenance, Repair \& Services: } \\
\hline 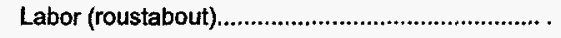 & 200.0 & 200.0 & 207.4 & 207.4 & 11,200 \\
\hline 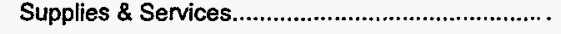 & 255.9 & 258.8 & 266.2 & 266.2 & 18,100 \\
\hline Equipment Usage & 231.3 & 231.3 & 237.5 & 262.5 & 4,200 \\
\hline Subtotal or Index & 231.2 & 232.6 & 239.9 & 242.8 & 33,500 \\
\hline \multicolumn{6}{|l|}{ Subsurface Maintenance, Repair \& Services: } \\
\hline 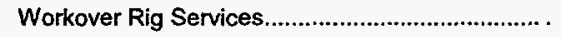 & 184.2 & 189.5 & 189.5 & 189.5 & 3,600 \\
\hline 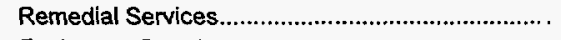 & 214.0 & 219.3 & 224.6 & 229.8 & 13,100 \\
\hline 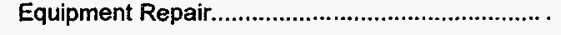 & 368.9 & 392.2 & 418.4 & 421.4 & 43,400 \\
\hline 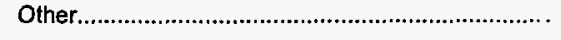 & 166.7 & 166.7 & 166.7 & 166.7 & 1,500 \\
\hline Subtotal or Index ${ }^{\star *}$ & 293.6 & 308.5 & 324.5 & 327.7 & 61,600 \\
\hline Total or Index ${ }^{* \pm} \ldots$ & 380.1 & 371.4 & 371.7 & 367.6 & 340,400 \\
\hline \multirow{2}{*}{ Component } & \multicolumn{4}{|c|}{ Index $(1976=100)$} & $1997^{*}$ \\
\hline & 1994 & 1995 & 1996 & 1997 & (dollars) \\
\hline \multicolumn{6}{|l|}{ Normal Daily Expense: } \\
\hline 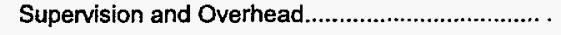 & 313.3 & 336.7 & 339.8 & 356.1 & 34,900 \\
\hline Labor (pumper). & 276.0 & 276.0 & 276.0 & 284.4 & 27,300 \\
\hline Auto Usage & 272.0 & 284.0 & 296.0 & 308.0 & 7,700 \\
\hline 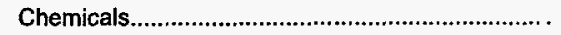 & 208.6 & 208.6 & 208.6 & 220.0 & 7,700 \\
\hline 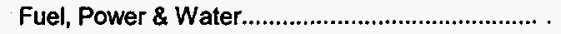 & 552.0 & 513.7 & 503.4 & 482.0 & 268,000 \\
\hline 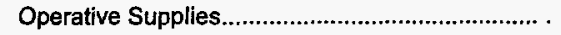 & 191.7 & 200.0 & 208.3 & 208.3 & 2,500 \\
\hline Subtotal or Index ${ }^{\star \star} \ldots$ & 462.9 & 440.3 & 434.2 & 423.5 & 348,100 \\
\hline \multicolumn{6}{|l|}{ Surface Maintenance, Repair \& Services: } \\
\hline 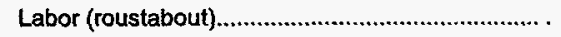 & 200.0 & 200.0 & 207.4 & 207.4 & 11,200 \\
\hline 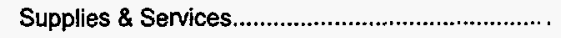 & 236.0 & 238.4 & 244.2 & 245.3 & 21,100 \\
\hline 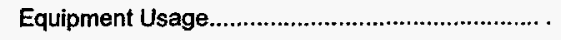 & 231.3 & 231.3 & 237.5 & 262.5 & 4,200 \\
\hline Subtotal or Index ${ }^{\star *}$ & 223.1 & 224.4 & 230.8 & 234.0 & 36,500 \\
\hline \multicolumn{6}{|l|}{ Subsurface Maintenance, Repair \& Services: } \\
\hline 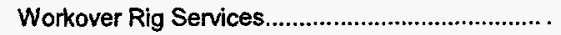 & 192.9 & 192.9 & 196.4 & 196.4 & 5,500 \\
\hline 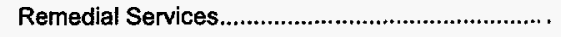 & 179.0 & 183.8 & 187.6 & 191.4 & 20,100 \\
\hline 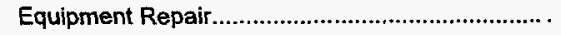 & 411.7 & 430.2 & 460.3 & 460.3 & 82,400 \\
\hline 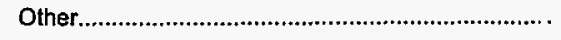 & 153.8 & 153.8 & 153.8 & 153.8 & 2,000 \\
\hline Subtotal or Index ${ }^{* *} \ldots$ & 307.4 & 319.1 & 337.2 & 338.5 & 110,000 \\
\hline Total or Index ${ }^{* \star} \ldots \ldots$ & 395.4 & $\mathbf{3 8 4 . 2}$ & 385.6 & 379.6 & 494,600 \\
\hline
\end{tabular}


Table G1. Annual Operating Costs and Indices for a 12-Slot Platform in the Gulf of Mexico 100-Foot Water Depth

\begin{tabular}{|c|c|c|c|c|c|}
\hline \multirow{2}{*}{ Component } & \multicolumn{4}{|c|}{ Index $(1976=100)$} & \multirow{2}{*}{$\begin{array}{c}1997^{*} \\
\text { Cost } \\
\text { (dollars) }\end{array}$} \\
\hline & 1994 & 1995 & 1996 & 1997 & \\
\hline 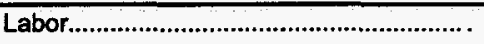 & 261.9 & 272.4 & 272.4 & 283.3 & 654,500 \\
\hline Supervision ..................................................... & 262.4 & 272.8 & 272.8 & 283.8 & 98,200 \\
\hline Payroll Overhead........................................... . & 349.3 & 363.2 & 363.2 & 377.8 & 301,100 \\
\hline Food Expense............................................. & 153.8 & 183.7 & 189.7 & 199.2 & 79,300 \\
\hline Labor Transportation................................. & 259.4 & 259.4 & 259.4 & 280.0 & 495,100 \\
\hline Surface Equipment................................. . & 227.7 & 224.6 & 229.3 & 233.9 & 115,800 \\
\hline Operating Supplies.................................... . & 227.3 & 224.2 & 229.3 & 234.3 & 23,200 \\
\hline 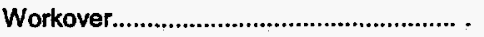 & 282.8 & 286.4 & 313.6 & 312.8 & 855,300 \\
\hline Communications.......................................... . & 479.1 & 479.1 & 479.1 & 479.1 & 20,600 \\
\hline Administrative & 255.3 & 263.0 & 264.0 & 273.6 & 317,400 \\
\hline 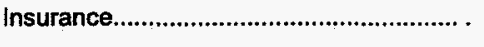 & 123.3 & 144.9 & 144.9 & 101.4 & 217,500 \\
\hline 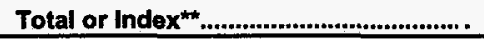 & 242.7 & 252.0 & 258.5 & 258.5 & $3,178,000$ \\
\hline
\end{tabular}

Table G2. Annual Operating Costs and Indices for a 12-Slot Platform in the Gulf of Mexico 300-Foot Water Depth

\begin{tabular}{|c|c|c|c|c|c|}
\hline \multirow{2}{*}{ Component } & \multicolumn{4}{|c|}{ Index $(1976=100)$} & \multirow{2}{*}{$\begin{array}{c}1997^{*} \\
\text { Cost } \\
\text { (dollars) }\end{array}$} \\
\hline & 1994 & 1995 & 1996 & 1997 & \\
\hline 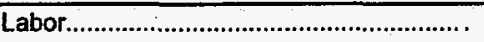 & 261.9 & 272.4 & 272.4 & 283.3 & 654,500 \\
\hline 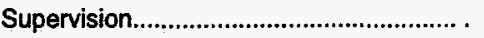 & 262.4 & 272.8 & 272.8 & 283.8 & 98,200 \\
\hline Payroll Overhead........................................ . & 349.3 & 363.2 & 363.2 & 377.8 & 301,100 \\
\hline 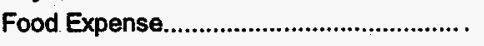 & 153.8 & 183.7 & 189.7 & 199.2 & 79,300 \\
\hline Labor Transportation................................... & 263.2 & 263.2 & 263.2 & 282.2 & 541,900 \\
\hline Surface Equipment..................................... . & 227.8 & 224.6 & 229.4 & 234.0 & 117,000 \\
\hline Operating Supplies...................................... & 228.0 & 225.0 & 229.0 & 234.0 & 23,400 \\
\hline 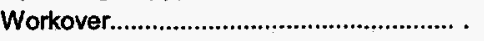 & 288.3 & 291.8 & 319.5 & 318.7 & 911,600 \\
\hline 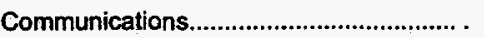 & 93.1 & 93.1 & 93.1 & 93.1 & 28,400 \\
\hline 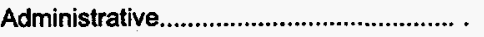 & 255.1 & 262.8 & 263.8 & 273.4 & 318,000 \\
\hline 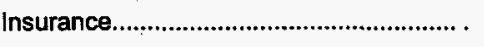 & 122.4 & 143.9 & 143.9 & 100.7 & 245,200 \\
\hline Total or Index & 238.0 & 247.1 & 253.6 & 252.7 & $3,318,600$ \\
\hline
\end{tabular}

Table G3. Annual Operating Costs and Indices for a 18-Slot Platform in the Gulf of Mexico 100-Foot Water Depth

\begin{tabular}{|c|c|c|c|c|c|}
\hline \multirow{2}{*}{ Component } & \multicolumn{4}{|c|}{ Index $(1976=100)$} & \multirow{2}{*}{$\begin{array}{c}1997^{\star} \\
\text { Cost } \\
\text { (dollars) }\end{array}$} \\
\hline & 1994 & 1995 & 1996 & 1997 & \\
\hline 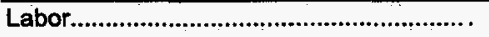 & 253.6 & 263.8 & 263.8 & 274.3 & 721,400 \\
\hline 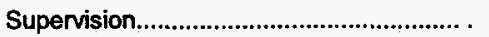 & 254.1 & 264.2 & 264.2 & 274.6 & 108,200 \\
\hline Payroll Overhead........................................ & 337.9 & 351.4 & 351.4 & 365.4 & 331,800 \\
\hline 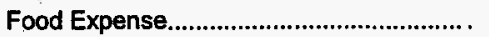 & 153.6 & 183.5 & 189.5 & 199.1 & 90,600 \\
\hline Labor Transportation.................................... & 259.4 & 259.4 & 259.4 & 259.4 & 458,600 \\
\hline Surface Equipment..................................... . & 205.8 & 203.1 & 207.2 & 211.6 & 117,000 \\
\hline 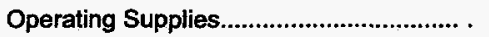 & 205.4 & 202.7 & 206.3 & 210.8 & 23,400 \\
\hline 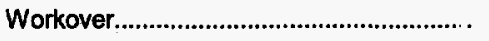 & 287.2 & 290.9 & 318.5 & 317.7 & $1,282,900$ \\
\hline 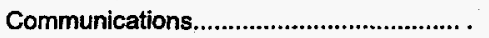 & 167.5 & 167.5 & 167.5 & 167.5 & 20,600 \\
\hline 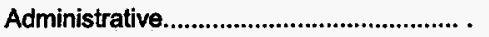 & 244.6 & 252.2 & 253.0 & 262.3 & 344,700 \\
\hline 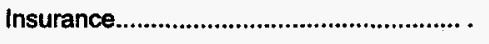 & 122.3 & 143.6 & 143.6 & 100.6 & 318,900 \\
\hline Total or Index & 234.6 & 244.2 & 251.8 & 246.9 & $3,818,100$ \\
\hline
\end{tabular}


Table G4. Annual Operating Costs and Indices for a 18-Slot Platform in the Gulf of Mexico 300-Foot Water Depth

\begin{tabular}{|c|c|c|c|c|c|}
\hline \multirow{2}{*}{ Component } & \multicolumn{4}{|c|}{ Index $(1976=100)$} & \multirow{2}{*}{$\begin{array}{c}1997^{*} \\
\text { Cost } \\
\text { (dollars) }\end{array}$} \\
\hline & 1994 & 1995 & 1996 & 1997 & \\
\hline 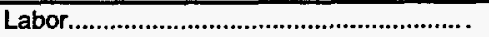 & 253.6 & 263.8 & 263.8 & 274.3 & 721,400 \\
\hline 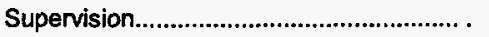 & 254.1 & 264.2 & 264.2 & 274.6 & 108,200 \\
\hline Payroll Overhead.......................................... & 338.3 & 351.8 & 351.8 & 365.8 & 331,800 \\
\hline 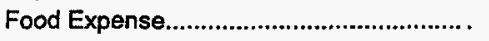 & 153.6 & 183.5 & 189.5 & 199.1 & 90,600 \\
\hline 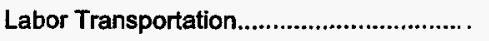 & 213.1 & 213.1 & 213.1 & 213.1 & 409,200 \\
\hline Surface Equipment.................................... & 203.6 & 200.9 & 205.0 & 209.3 & 117,000 \\
\hline Operating Supplies......................................... . & 203.6 & 200.9 & 204.5 & 208.9 & 23,400 \\
\hline 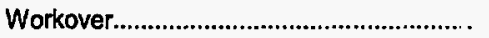 & 292.5 & 296.0 & 324.1 & 323.3 & $1,367,300$ \\
\hline 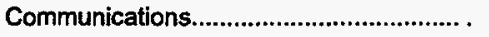 & 93.1 & 93.1 & 93.1 & 93.1 & 28,400 \\
\hline 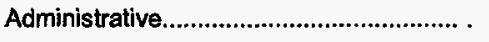 & 243.9 & 251.4 & 252.3 & 261.5 & 344,700 \\
\hline 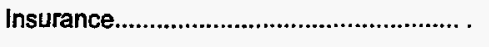 & 121.1 & 142.3 & 142.3 & 99.6 & 340,300 \\
\hline Total or Index & 227.1 & 236.5 & 244.2 & 239.0 & $3,882,300$ \\
\hline
\end{tabular}

Table G5. Annual Operating Costs and Indices for a 18-Slot Platform in the Gulf of Mexico 600-Foot Water Depth

\begin{tabular}{|c|c|c|c|c|c|}
\hline \multirow{2}{*}{ Component } & \multicolumn{4}{|c|}{ Index $(1976=100)$} & \multirow{2}{*}{$\begin{array}{c}1997^{*} \\
\text { Cost } \\
\text { (dollars) }\end{array}$} \\
\hline & 1994 & 1995 & 1996 & 1997 & \\
\hline 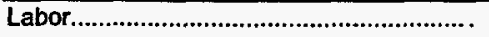 & 253.6 & 263.8 & 263.8 & 274.3 & 721,400 \\
\hline 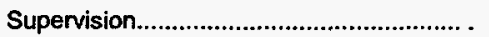 & 254.1 & 264.2 & 264.2 & 274.6 & 108,200 \\
\hline 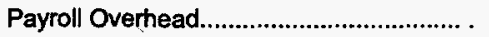 & 338.3 & 351.8 & 351.8 & 365.8 & 331,800 \\
\hline 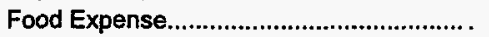 & 153.6 & 183.5 & 189.5 & 199.1 & 90,600 \\
\hline Labor Transportation.................................... & 255.7 & 255.7 & 255.7 & 255.7 & 523,080 \\
\hline Surface Equipment..................................... & 230.0 & 227.0 & 231.6 & 236.3 & 133,300 \\
\hline Operating Supplies....................................... & 229.2 & 226.5 & 231.0 & 236.3 & 26,700 \\
\hline 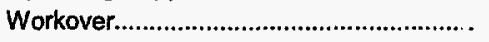 & 282.2 & 286.6 & 313.5 & 313.1 & $1,403,500$ \\
\hline 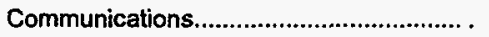 & 88.3 & 88.3 & 88.3 & 88.3 & 32,300 \\
\hline Administrative & 249.1 & 256.5 & 257.5 & 266.9 & 352,600 \\
\hline 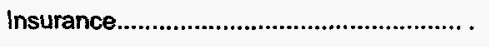 & 128.6 & 151.1 & 151.1 & 105.7 & 547,200 \\
\hline Total or Index & 222.9 & 233.8 & 240.7 & 231.4 & $4,270,680$ \\
\hline
\end{tabular}




\section{Section II}

\section{Appendices H Through M}

Costs and Indices for Domestic Gas Field

Equipment and Production Operations 



\section{Appendices H Through M}

\section{Costs and Indices for Domestic Gas Field Equipment and Production Operations}

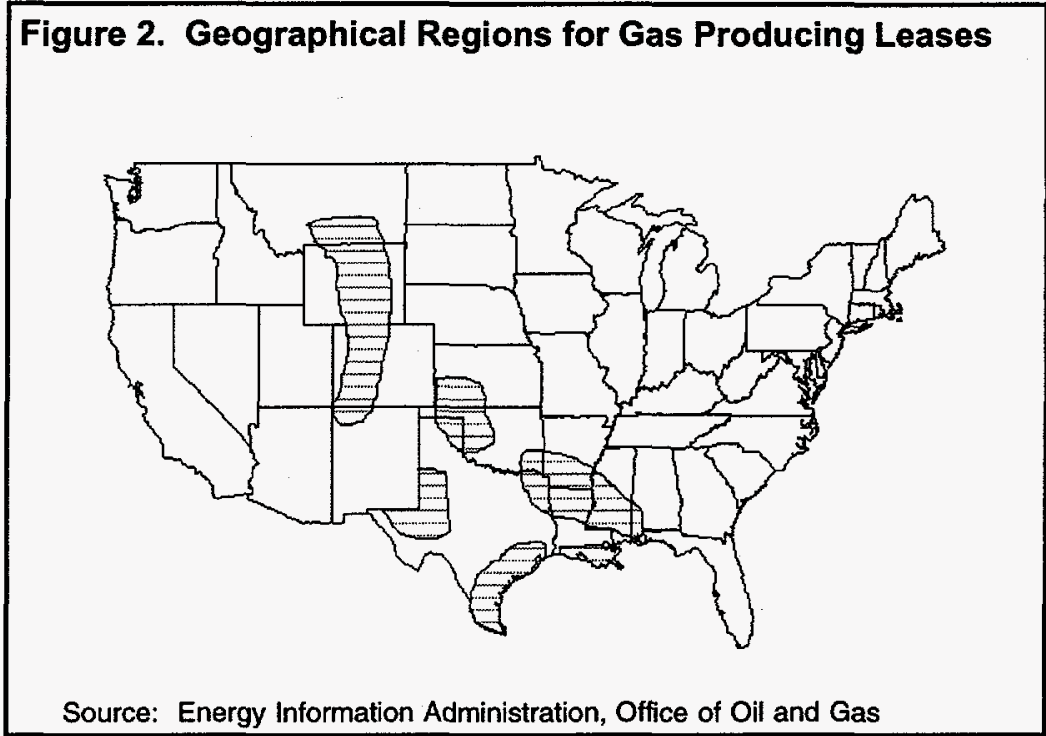

Appendices $\mathrm{H}$ through $\mathrm{M}$ contain details for gas leases. A detailed breakdown of 1997 costs and indices for 1994 through 1997 is shown in each of the gas lease tables. The tables are arranged by region with each region identified by an alpha character. Each table within the appendix is for a different depth. For example, Table $\mathrm{H} 1$ contains equipping cost data for west Texas gas leases at 2,000-foot depths; Table $\mathrm{H} 2$ contains equipping cost data for gas leases at 4,000-foot depths; Table H5 contains equipping cost data for 16,000-foot wells. Tables $\mathrm{H} 6$ through $\mathrm{H} 10$ contain operating costs for gas wells at 2,000,4,000, 8,000, 12,000 and 16,000 -foot depths, respectively. Each table is further divided into costs associated with different flow rates. For example, Table $\mathrm{H} 1$ has equipping costs for production rates of 50 and 250 thousand cubic feet per day only. Table H11 is a typical equipment list for a 12,000-foot gas well produc ing 1 million cubic feet per day in west Texas.

The remaining tables of costs and indices for gas leases by region are arranged in similar order. These appendices are: Appendix I--south Texas, Appendix J--south Louisiana, Appendix K--north Louisiana, Appendix L-- Mid-Continent, and Appendix M--Rocky Mountain Region.

Notes: • 1997 data are preliminary and are marked with a single asterisk $\left({ }^{*}\right)$. - All prior data were revised. • Indices marked with a double asterisk $\left({ }^{* *}\right)$ are composite indices. $\bullet$ Other indices are pure cost. 
Table H1. Lease Equipment Costs and Indices for Gas Production in West Texas (1 Well Producing from 2,000 Feet)

\begin{tabular}{|c|c|c|c|c|c|}
\hline \multirow{2}{*}{ Component } & \multicolumn{4}{|c|}{ Index $(1976=100)$} & \multirow{2}{*}{$\begin{array}{c}1997^{\star} \\
\text { Cost } \\
\text { (dollars) }\end{array}$} \\
\hline & 1994 & 1995 & 1996 & 1997 & \\
\hline & \multicolumn{5}{|c|}{50 Thousand Cubic Feet Per Day } \\
\hline Flowlines and Connections............................. & 261.5 & 269.2 & 269.2 & 284.6 & 3,700 \\
\hline Production Package & 170.6 & 170.6 & 114.7 & 114.7 & 3,900 \\
\hline 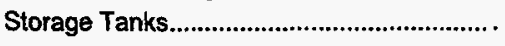 & 166.7 & 174.1 & 177.8 & 183.3 & 9,900 \\
\hline \multirow[t]{2}{*}{ Total or Index } & 180.2 & 185.1 & 168.3 & 173.3 & 17,500 \\
\hline & \multicolumn{5}{|c|}{250 Thousand Cubic Feet Per Day } \\
\hline Flowlines and Connections.............................. & 261.5 & 269.2 & 269.2 & 284.6 & 3,700 \\
\hline Production Package. & 170.6 & 170.6 & 114.7 & 114.7 & 3,900 \\
\hline 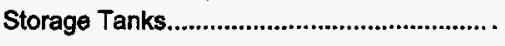 & 166.7 & 174.1 & 177.8 & 183.3 & 9,900 \\
\hline 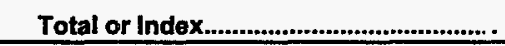 & 180.2 & 185.1 & 168.3 & 173.3 & 17,500 \\
\hline
\end{tabular}

Table H2. Lease Equipment Costs and Indices for Gas Production in West Texas (1 Well Producing from 4,000 Feet)

\begin{tabular}{|c|c|c|c|c|c|}
\hline \multirow{2}{*}{ Component } & \multicolumn{4}{|c|}{ Index $(1976=100)$} & \multirow{2}{*}{$\begin{array}{c}1997^{*} \\
\text { Cost } \\
\text { (dollars) }\end{array}$} \\
\hline & 1994 & 1995 & 1996 & 1997 & \\
\hline & \multicolumn{5}{|c|}{50 Thousand Cubic Feet Per Day } \\
\hline Flowlines and Connections........................... & 261.5 & 269.2 & 269.2 & 284.6 & 3,700 \\
\hline 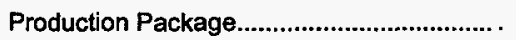 & 170.6 & 170.6 & 114.7 & 114.7 & 3,900 \\
\hline 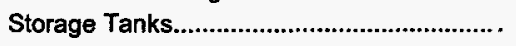 & 166.7 & 174.1 & 177.8 & 183.3 & 9,900 \\
\hline \multirow[t]{2}{*}{ 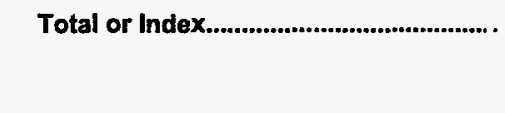 } & 180.2 & 185.1 & 168.3 & 173.3 & 17,500 \\
\hline & \multicolumn{5}{|c|}{250 Thousand Cubic Feet Per Day } \\
\hline Flowlines and Connections............................. & 211.1 & 222.2 & 233.3 & 244.4 & 11,000 \\
\hline 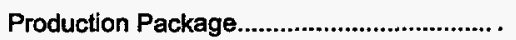 & 214.3 & 211.4 & 160.0 & 160.0 & 5,600 \\
\hline 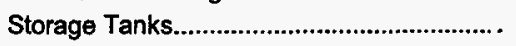 & 166.7 & 174.1 & 177.8 & 183.3 & 9,900 \\
\hline Total or Index. & 194.0 & 200.0 & 191.8 & 197.8 & 26,500 \\
\hline
\end{tabular}


Table H3. Lease Equipment Costs and Indices for Gas Production in West Texas (1 Well Producing from 8,000 Feet)

\begin{tabular}{|c|c|c|c|c|c|}
\hline \multirow{2}{*}{ Component } & \multicolumn{4}{|c|}{ Index $(1976=100)$} & \multirow{2}{*}{$\begin{array}{c}1997^{*} \\
\text { Cost } \\
\text { (dollars) }\end{array}$} \\
\hline & 1994 & 1995 & 1996 & 1997 & \\
\hline & \multicolumn{5}{|c|}{50 Thousand Cubic Feet Per Day } \\
\hline Flowlines and Connections............................. . & 208.3 & 220.8 & 231.3 & 241.7 & 11,600 \\
\hline 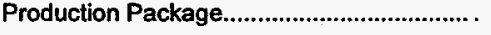 & 200.0 & 200.0 & 134.5 & 134.5 & 3,900 \\
\hline Storage Tanks................................................. & 166.7 & 174.1 & 177.8 & 183.3 & 9,900 \\
\hline \multirow[t]{2}{*}{ 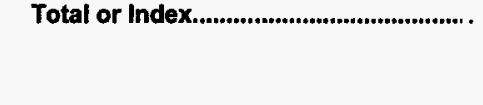 } & 189.3 & 196.9 & 187.8 & 193.9 & 25,400 \\
\hline & \multicolumn{5}{|c|}{250 Thousand Cubic Feet Per Day } \\
\hline Flowlines and Connections............................ . & 208.3 & 220.8 & 231.3 & 241.7 & 11,600 \\
\hline 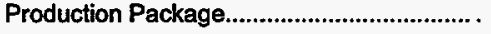 & 214.3 & 211.4 & 160.0 & 160.0 & 5,600 \\
\hline 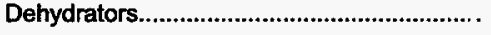 & 158.1 & 157.0 & 165.6 & 174.2 & 16,200 \\
\hline 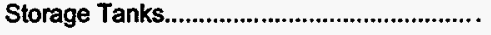 & 166.7 & 174.1 & 177.8 & 183.3 & 9,900 \\
\hline \multirow[t]{2}{*}{ 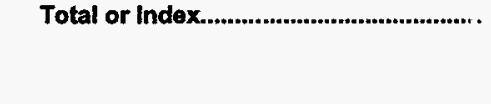 } & 179.1 & 182.6 & 181.3 & 188.3 & 43,300 \\
\hline & \multicolumn{5}{|c|}{500 Thousand Cubic Feet Per Day } \\
\hline Flowlines and Connections.... & 202.4 & 217.1 & 229.3 & 241.5 & 9,900 \\
\hline Production Package............ & 187.5 & 185.0 & 140.0 & 140.0 & 5,600 \\
\hline Dehydrators....................... & 158.1 & 157.0 & 165.6 & 174.2 & 16,200 \\
\hline Storage Tanks..................... & 166.7 & 174.1 & 177.8 & 183.3 & 9,900 \\
\hline 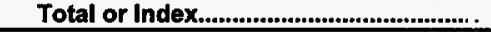 & 173.2 & 176.8 & 175.4 & 182.5 & 41,600 \\
\hline
\end{tabular}

Table H4. Lease Equipment Costs and Indices for Gas Production in West Texas (1 Well Producing from 12,000 Feet)

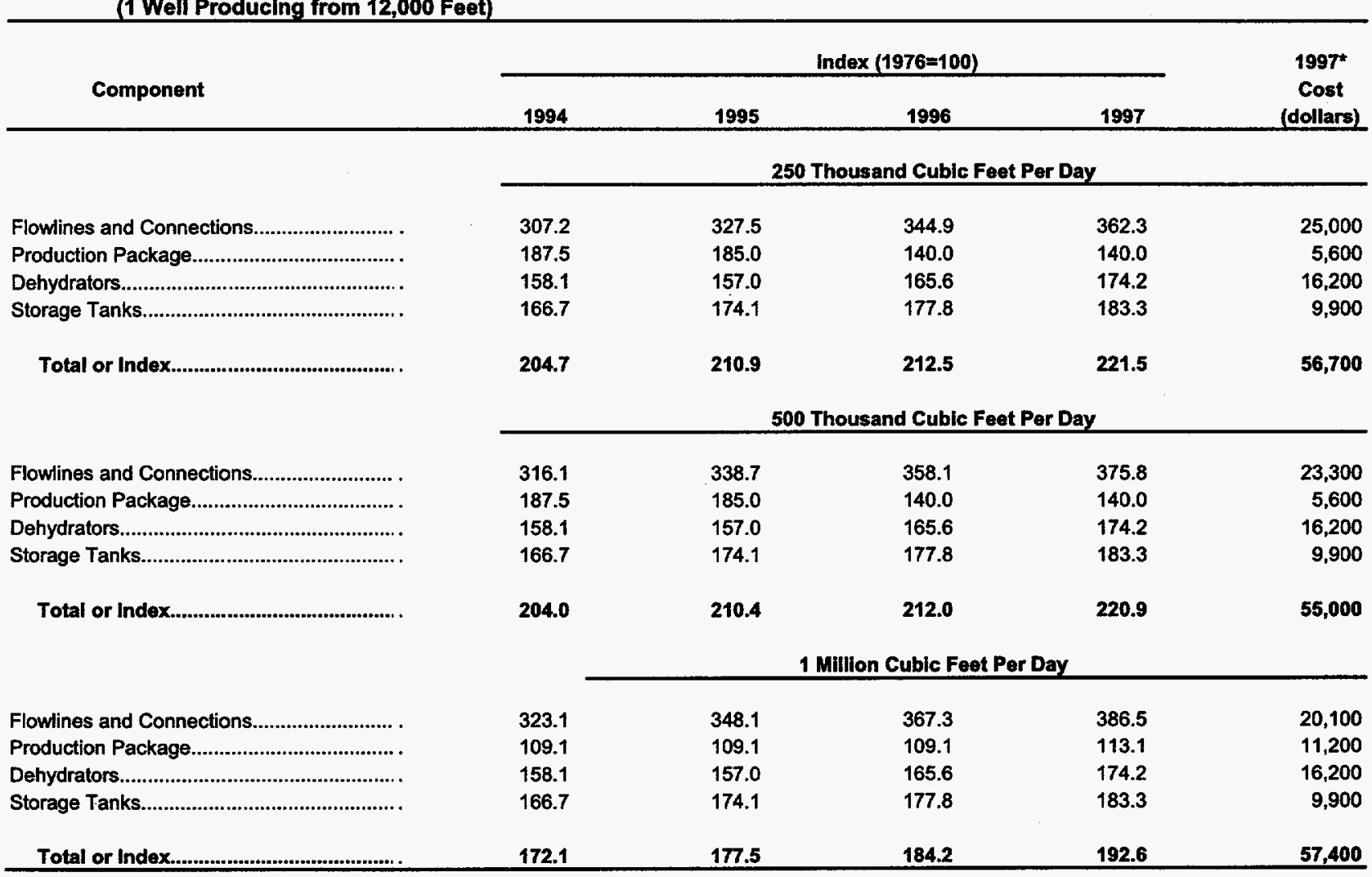


Table H5. Lease Equipment Costs and Indices for Gas Production in West Texas (1 Well Producing from 16,000 Feet)

\begin{tabular}{|c|c|c|c|c|c|}
\hline \multirow{2}{*}{ Component } & \multicolumn{4}{|c|}{ Index $(1976=100)$} & \multirow{2}{*}{$\begin{array}{c}1997^{\star} \\
\text { Cost } \\
\text { (dollars) }\end{array}$} \\
\hline & 1994 & 1995 & 1996 & 1997 & \\
\hline & \multicolumn{5}{|c|}{500 Thousand Cubic Feet Per Day } \\
\hline Flowlines and Connections.......................... . & 323.1 & 348.1 & 367.3 & 386.5 & 20,100 \\
\hline Production Package..................................... . & 109.1 & 109.1 & 109.1 & 113.1 & 11,200 \\
\hline 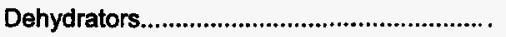 & 158.1 & 157.0 & 165.6 & 174.2 & 16,200 \\
\hline Storage Tanks................................................... & 166.7 & 174.1 & 177.8 & 183.3 & 9,900 \\
\hline \multirow[t]{2}{*}{ Total or Index............................................ . } & 172.1 & 177.5 & 184.2 & 192.6 & 57,400 \\
\hline & \multicolumn{5}{|c|}{1 Million Cubic Feet Per Day } \\
\hline Flowlines and Connections.......................... & 323.1 & 348.1 & 367.3 & 386.5 & 20,100 \\
\hline Production Package ....................................... & 109.1 & 109.1 & 109.1 & 113.1 & 11,200 \\
\hline 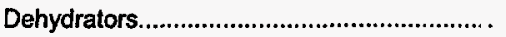 & 158.1 & 157.0 & 165.6 & 174.2 & 16,200 \\
\hline 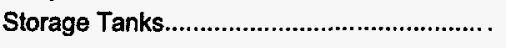 & 166.7 & 174.1 & 177.8 & 183.3 & 9,900 \\
\hline \multirow[t]{2}{*}{ 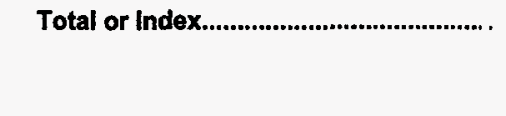 } & 172.1 & 177.5 & 184.2 & 192.6 & 57,400 \\
\hline & \multicolumn{5}{|c|}{5 Million Cubic Feet Per Day } \\
\hline Flowlines and Connections........................... . & 301.2 & 323.8 & 342.7 & 359.8 & 59,000 \\
\hline Production Package...................................... . & 129.6 & 123.1 & 120.4 & 124.1 & 13,400 \\
\hline 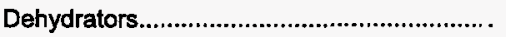 & 187.6 & 187.6 & 187.6 & 205.3 & 23,200 \\
\hline Storage Tanks............................................... & 166.7 & 174.1 & 177.8 & 183.3 & 9,900 \\
\hline Total or Index............................................... . & 213.2 & 221.0 & 227.8 & 240.3 & 105,500 \\
\hline
\end{tabular}

Table H6. Direct Annual Operating Costs and Indices for Gas Production in West Texas (1 Well Producing from 2,000 Feet)

\begin{tabular}{|c|c|c|c|c|c|}
\hline \multirow{2}{*}{ Component } & \multicolumn{4}{|c|}{ Index $(1976=100)$} & \multirow{2}{*}{$\begin{array}{c}1997^{\star} \\
\text { Cost } \\
\text { (dollars) }\end{array}$} \\
\hline & 1994 & 1995 & 1996 & 1997 & \\
\hline & \multicolumn{5}{|c|}{50 Thousand Cubic Feet Per Day } \\
\hline Direct Labor \& Overhead................................ & 283.3 & 291.7 & 291.7 & 300.0 & 3,600 \\
\hline Fuel, Chemicals \& Disposal......................... . & 300.0 & 300.0 & 300.0 & 300.0 & 600 \\
\hline Surface Maintenance.................................... . & 240.0 & 240.0 & 233.3 & 233.3 & 3,500 \\
\hline Subsurface Maintenance................................ . & 180.0 & 180.0 & 180.0 & 180.0 & 900 \\
\hline \multirow[t]{2}{*}{ 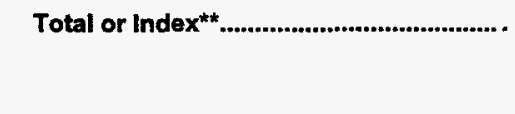 } & 250.0 & 252.9 & 250.0 & 252.9 & $B, 600$ \\
\hline & \multicolumn{5}{|c|}{250 Thousand Cubic Feet Per Day } \\
\hline Direct Labor \& Overhead................................. . & 283.3 & 291.7 & 291.7 & 300.0 & 3,600 \\
\hline Fuel, Chemicals \& Disposal......................... . & 220.0 & 220.0 & 220.0 & 240.0 & 2,400 \\
\hline Surface Maintenance....................................... . & 240.0 & 240.0 & 233.3 & 233.3 & 3,500 \\
\hline Subsurface Maintenance................................ & 180.0 & 180.0 & 180.0 & 180.0 & 900 \\
\hline 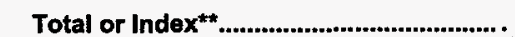 & 240.5 & 242.9 & 240.5 & 247.6 & 10,400 \\
\hline
\end{tabular}


Table H7. Direct Annual Operating Costs and Indices for Gas Production in West Texas (1 Well Producing from 4,000 Feet)

\begin{tabular}{|c|c|c|c|c|c|}
\hline \multirow{2}{*}{ Component } & \multicolumn{4}{|c|}{ Index $(1976=100)$} & \multirow{2}{*}{$\begin{array}{c}1997^{*} \\
\text { Cost } \\
\text { (dollars) }\end{array}$} \\
\hline & 1994 & 1995 & 1996 & 1997 & \\
\hline & \multicolumn{5}{|c|}{50 Thousand Cubic Feet Per Day } \\
\hline Direct Labor \& Overhead.................................. . & 271.4 & 278.6 & 285.7 & 292.9 & 4,100 \\
\hline Fuel, Chemicals \& Disposal.......................... & 220.0 & 220.0 & 220.0 & 240.0 & 1,200 \\
\hline Surface Maintenance..................................... & 266.7 & 266.7 & 266.7 & 266.7 & 4,000 \\
\hline Subsurface Maintenance............................... & 185.7 & 185.7 & 185.7 & 185.7 & 1,300 \\
\hline \multirow[t]{2}{*}{ Total or Index ${ }^{\star \star}$} & 248.8 & 251.2 & 253.7 & 258.5 & 10,600 \\
\hline & \multicolumn{5}{|c|}{250 Thousand Cubic Feet Per Day } \\
\hline Direct Labor \& Overhead................................ . & 271.4 & 278.6 & 285.7 & 292.9 & 4,100 \\
\hline Fuel, Chemicals \& Disposal......................... & 220.0 & 220.0 & 220.0 & 235.0 & 4,700 \\
\hline Surface Maintenance...................................... & 225.0 & 230.0 & 225.0 & 225.0 & 4,500 \\
\hline Subsurface Maintenance............................... . & 185.7 & 185.7 & 185.7 & 185.7 & 1,300 \\
\hline 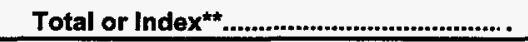 & 229.5 & 232.8 & 232.8 & 239.3 & 14,600 \\
\hline
\end{tabular}

Table H8. Direct Annual Operating Costs and Indices for Gas Production in West Texas (1 Well Producing from 8,000 Feet)

\begin{tabular}{|c|c|c|c|c|c|}
\hline \multirow{2}{*}{ Component } & \multicolumn{4}{|c|}{ Index $(1976=100)$} & \multirow{2}{*}{$\begin{array}{c}\text { 1997* } \\
\text { Cost } \\
\text { (dollars) }\end{array}$} \\
\hline & 1994 & 1995 & 1996 & 1997 & \\
\hline & \multicolumn{5}{|c|}{50 Thousand Cubic Feet Per Day } \\
\hline Direct Labor \& Overhead................................ . & 275.0 & 287.5 & 287.5 & 300.0 & 4,800 \\
\hline Fuel, Chemicals \& Disposal........................ . & 220.0 & 220.0 & 220.0 & 240.0 & 2,400 \\
\hline Surface Maintenance..................................... . & 225.0 & 225.0 & 220.0 & 225.0 & 4,500 \\
\hline Subsurface Maintenance............................... . & 166.7 & 166.7 & 166.7 & 175.0 & 2,100 \\
\hline \multirow[t]{2}{*}{ Total or Index ${ }^{* *} \ldots \ldots \ldots \ldots \ldots \ldots \ldots$} & 225.9 & 229.3 & 227.6 & 237.9 & 13,800 \\
\hline & \multicolumn{5}{|c|}{250 Thousand Cubic Feet Per Day } \\
\hline Direct Labor \& Overhead................................ . & 275.0 & 287.5 & 287.5 & 300.0 & 4,800 \\
\hline Fuel, Chemicals \& Disposal.......................... & 228.2 & 228.2 & 228.2 & 243.6 & 9,500 \\
\hline Surface Maintenance..................................... . & 220.5 & 223.1 & 220.5 & 223.1 & 8,700 \\
\hline Subsurface Maintenance................................. . & 166.7 & 166.7 & 166.7 & 175.0 & 2,100 \\
\hline \multirow[t]{2}{*}{ Total or Index } & 225.5 & 228.3 & 227.4 & 236.8 & 25,100 \\
\hline & \multicolumn{5}{|c|}{500 Thousand Cublc Feet Per Day } \\
\hline Direct Labor \& Overhead................................. . & 275.0 & 287.5 & 287.5 & 300.0 & 4,800 \\
\hline Fuel, Chemicals \& Disposal........................ . & 238.1 & 233.3 & 238.1 & 242.9 & 5,100 \\
\hline Surface Maintenance...................................... . & 220.6 & 220.6 & 220.6 & 220.6 & 7,500 \\
\hline Subsurface Maintenance................................ . & 166.7 & 166.7 & 166.7 & 175.0 & 2,100 \\
\hline 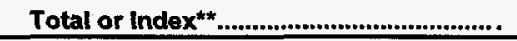 & 227.7 & 228.9 & 230.1 & 234.9 & 19,500 \\
\hline
\end{tabular}


Table H9. Direct Annual Operating Costs and Indices for Gas Production in West Texas (1 Well Producing from 12,000 Feet)

\begin{tabular}{|c|c|c|c|c|c|}
\hline \multirow{2}{*}{ Component } & \multicolumn{4}{|c|}{ Index $(1976=100)$} & \multirow{2}{*}{$\begin{array}{c}1997^{*} \\
\text { Cost } \\
\text { (dollars) }\end{array}$} \\
\hline & 1994 & 1995 & 1996 & 1997 & \\
\hline & \multicolumn{5}{|c|}{250 Thousand Cubic Feet Per Day } \\
\hline Direct Labor \& Overhead..................................... . & 278.9 & 294.7 & 294.7 & 305.3 & 5,800 \\
\hline Fuel, Chemicals \& Disposal........................... & 222.0 & 222.0 & 222.0 & 238.0 & 11,900 \\
\hline 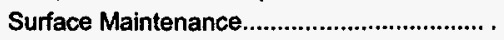 & 215.0 & 217.5 & 215.0 & 217.5 & 8,700 \\
\hline Subsurface Maintenance................................... . & 180.8 & 184.6 & 184.6 & 188.5 & 4,900 \\
\hline \multirow[t]{2}{*}{ Total or Index ${ }^{* *} \ldots$} & 220.0 & 223.7 & 223.0 & 231.9 & 31,300 \\
\hline & \multicolumn{5}{|c|}{500 Thousand Cubic Feet Per Day } \\
\hline Direct Labor \& Overhead.............................. . & 278.9 & 294.7 & 294.7 & 305.3 & 5,800 \\
\hline Fuel, Chemicals \& Disposal & 220.0 & 220.0 & 224.0 & 228.0 & 5,700 \\
\hline 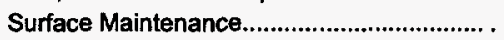 & 214.3 & 214.3 & 214.3 & 214.3 & 7,500 \\
\hline Subsurface Maintenance................................... . & 180.8 & 184.6 & 184.6 & 188.5 & 4,900 \\
\hline \multirow[t]{2}{*}{ Total or Index $x^{* *}$} & 219.0 & 222.9 & 223.8 & 227.6 & 23,900 \\
\hline & \multicolumn{5}{|c|}{1 Million Cubic Feet Per Day } \\
\hline Direct Labor \& Overhead............................... . & 278.9 & 294.7 & 294.7 & 305.3 & 5,800 \\
\hline Fuel, Chemicals \& Disposal.......................... & 225.0 & 223.1 & 228.8 & 234.6 & 12,200 \\
\hline 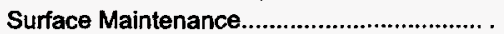 & 200.0 & 200.0 & 200.0 & 202.6 & 7,900 \\
\hline Subsurface Maintenance................................... . & 180.8 & 184.6 & 184.6 & 188.5 & 4,900 \\
\hline Total or Index & 216.9 & 219.1 & 221.3 & 226.5 & 30,800 \\
\hline
\end{tabular}

Table H10. Direct Annual Operating Costs and Indices for Gas Production in West Texas (1 Well Producing from 16,000 Feet)

\begin{tabular}{|c|c|c|c|c|c|}
\hline \multirow{2}{*}{ Component } & \multicolumn{4}{|c|}{ Index $(1976=100)$} & \multirow{2}{*}{$\begin{array}{c}\text { 1997* } \\
\text { Cost } \\
\text { (dollars) }\end{array}$} \\
\hline & 1994 & 1995 & 1996 & 1997 & \\
\hline & \multicolumn{5}{|c|}{500 Thousand Cubic Feet Per Day } \\
\hline Direct Labor \& Overhead.............................. . & 278.9 & 294.7 & 294.7 & 305.3 & 5,800 \\
\hline Fuel, Chemicals \& Disposal.......................... . & 222.6 & 219.4 & 225.8 & 232.3 & 7,200 \\
\hline Surface Maintenance .......................................... . & 215.4 & 217.9 & 217.9 & 220.5 & 8,600 \\
\hline Subsurface Maintenance................................ . & 177.4 & 180.6 & 180.6 & 183.9 & 5,700 \\
\hline \multirow[t]{2}{*}{ Total or Index } & 217.5 & 220.8 & 222.5 & 227.5 & 27,300 \\
\hline & \multicolumn{5}{|c|}{1 Million Cubic Feet Per Day } \\
\hline Direct Labor \& Overhead............................... . & 278.9 & 294.7 & 294.7 & 305.3 & 5,800 \\
\hline Fuel, Chemicals \& Disposal.......................... . & 219.7 & 219.7 & 223.0 & 229.5 & 14,000 \\
\hline Surface Maintenance...................................... . & 215.4 & 217.9 & 217.9 & 220.5 & 8,600 \\
\hline Subsurface Maintenance................................. & 177.4 & 180.6 & 180.6 & 183.9 & 5,700 \\
\hline \multirow[t]{2}{*}{ Total or Index } & 217.3 & 220.7 & 222.0 & 227.3 & 34,100 \\
\hline & \multicolumn{5}{|c|}{5 Million Cubic Feet Per Day } \\
\hline Direct Labor \& Overhead................................. . & 278.9 & 294.7 & 294.7 & 305.3 & 5,800 \\
\hline Fuel, Chemicals \& Disposal.......................... . & 153.5 & 153.5 & 163.6 & 167.7 & 16,600 \\
\hline Surface Maintenance...................................... . & 230.6 & 234.7 & 238.8 & 244.9 & 12,000 \\
\hline Subsurface Maintenance................................ . & 127.3 & 130.3 & 130.3 & 136.4 & 4,500 \\
\hline Total or Index & 180.0 & 183.0 & 189.0 & 194.5 & 38,900 \\
\hline
\end{tabular}


Table H11. Detailed Lease Equipment List for 12,000-Foot Gas Wells in West Texas Producing 1 Million Cubic Feet per Day

\section{Safety Valve}

Size: 2 inches

Working Pressure: 10,000 pounds per square inch

Actuates: High/low pressures

Production Package

Choke: Built in, inlet

Coils: 2 inch $\mathrm{XH}$

Heater rating: 250,000 BTU per hour

Size: 16 inches by 8 feet

Working pressure: 1,000 pounds per square inch
Dehydrator/Reconcentrator

Type: Glycol absorption

Size: $12-3 / 4$ inches

Working pressure: 1,440 pounds per square inch

Storage Tanks (2)

Size: 10 feet by 15 feet

Capacity 210 barrels

Construction: Welded steel

Source: Energy Information Administration, Office of Oil and Gas 
Table 11. Lease Equipment Costs and Indices for Gas Production in South Texas (1 Well Producing from 2,000 Feet)

\begin{tabular}{|c|c|c|c|c|c|}
\hline \multirow{2}{*}{ Component } & \multicolumn{4}{|c|}{ Index $(1976=100)$} & \multirow{2}{*}{$\begin{array}{c}1997^{*} \\
\text { Cost } \\
\text { (dolliars) }\end{array}$} \\
\hline & 1994 & 1995 & 1996 & 1997 & \\
\hline & \multicolumn{5}{|c|}{50 Thousand Cubic Feet Per Day } \\
\hline Flowlines and Connections............................. & 281.5 & 269.2 & 276.9 & 284.6 & 3,700 \\
\hline Production Package & 160.0 & 160.0 & 171.4 & 177.1 & 6,200 \\
\hline 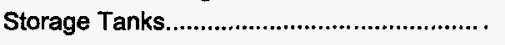 & 163.6 & 170.9 & 174.5 & 180.0 & 9,900 \\
\hline \multirow[t]{2}{*}{ 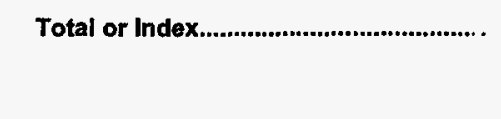 } & 174.8 & 179.6 & 186.4 & 192.2 & 19,800 \\
\hline & \multicolumn{5}{|c|}{250 Thousand Cubic Feet Per Day } \\
\hline Flowlines and Connections............................ . & 261.5 & 269.2 & 276.9 & 284.6 & 3,700 \\
\hline 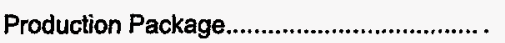 & 160.0 & 160.0 & 171.4 & 177.1 & 6,200 \\
\hline 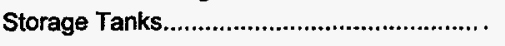 & 163.6 & 170.9 & 174.5 & 180.0 & 9,900 \\
\hline 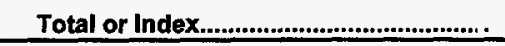 & 174.8 & 179.6 & 186.4 & 192.2 & 19,800 \\
\hline
\end{tabular}

Table 12. Lease Equipment Costs and Indices for Gas Production in South Texas (1 Well Producing from 4,000 Feet)

\begin{tabular}{|c|c|c|c|c|c|}
\hline \multirow{2}{*}{ Component } & \multicolumn{4}{|c|}{ Index $(1976=100)$} & \multirow{2}{*}{$\begin{array}{c}1997^{*} \\
\text { Cost } \\
\text { (dollars) }\end{array}$} \\
\hline & 1994 & 1995 & 1996 & 1997 & \\
\hline & \multicolumn{5}{|c|}{50 Thousand Cubic Feet Per Day } \\
\hline Flowlines and Connections............................... . & 261.5 & 269.2 & 276.9 & 284.6 & 3,700 \\
\hline Production Package & 160.0 & 160.0 & 171.4 & 177.1 & 6,200 \\
\hline 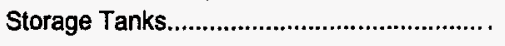 & 163.6 & 170.9 & 174.5 & 180.0 & 9,900 \\
\hline \multirow[t]{2}{*}{ 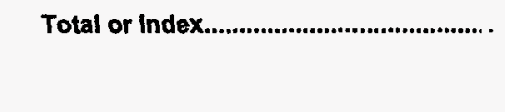 } & 174.8 & 179.6 & 186.4 & 192.2 & 19,800 \\
\hline & \multicolumn{5}{|c|}{250 Thousand Cubic Feet Per Day } \\
\hline Flowlines and Connections............................... & 208.7 & 219.6 & 230.4 & 239.1 & 11,000 \\
\hline Production Package....................................... & 197.3 & 197.3 & 210.8 & 216.2 & 8,000 \\
\hline 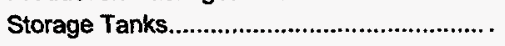 & 163.6 & 170.9 & 174.5 & 180.0 & 9,900 \\
\hline 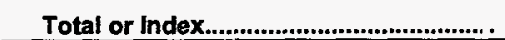 & 187.7 & 194.2 & 202.9 & 209.4 & 28,900 \\
\hline
\end{tabular}


Table 13. Lease Equipment Costs and Indices for Gas Production in South Texas (1 Well Producing from 8,000 Feet)

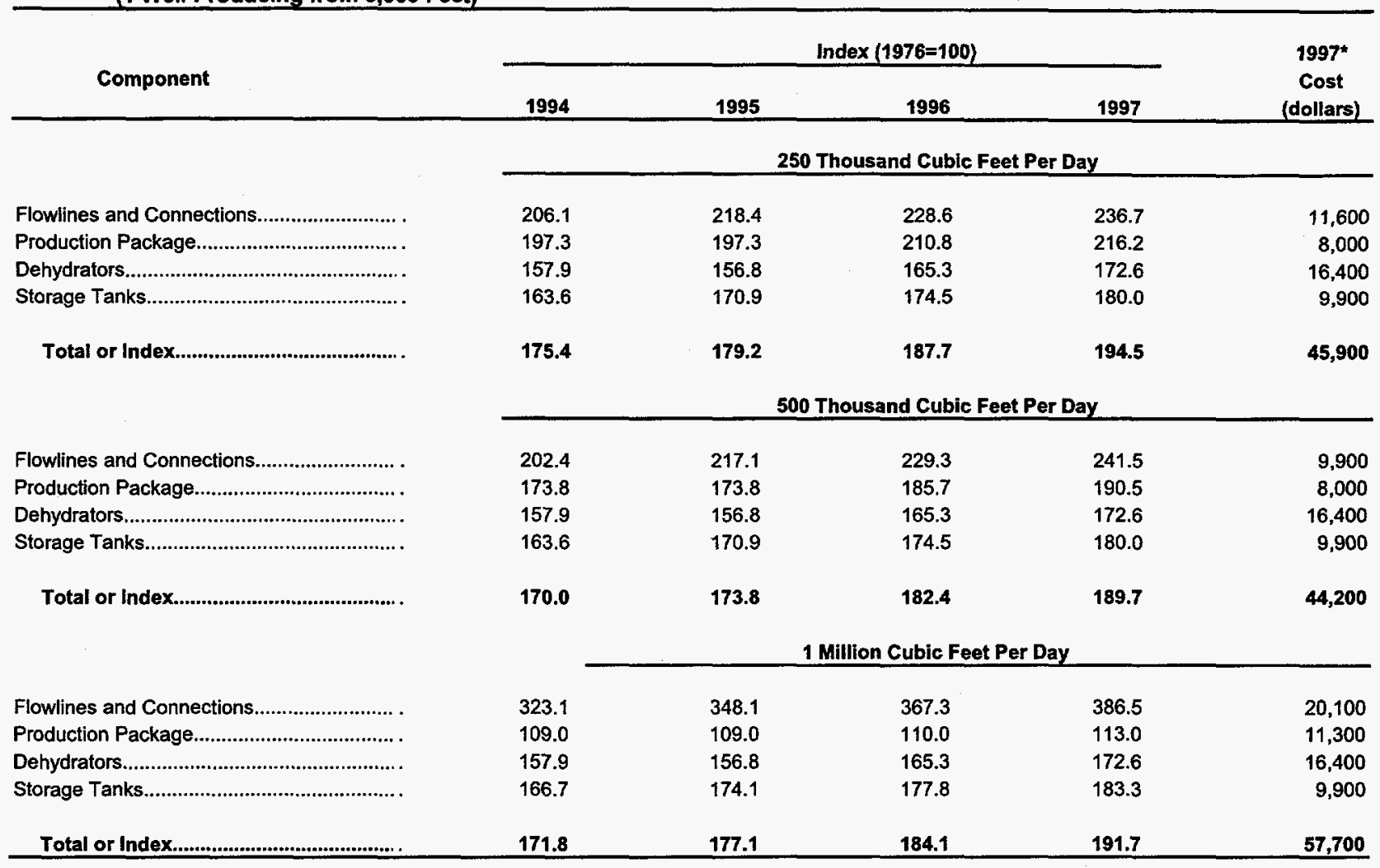

Table I4. Lease Equipment Costs and Indices for Gas Production in South Texas (1 Well Producing from 12,000 Feet)

\begin{tabular}{|c|c|c|c|c|c|}
\hline \multirow{2}{*}{ Component } & \multicolumn{4}{|c|}{ Index $(1976=100)$} & \multirow{2}{*}{$\begin{array}{c}\text { 1997* } \\
\text { Cost } \\
\text { (dollars) }\end{array}$} \\
\hline & 1994 & 1995 & 1996 & 1997 & \\
\hline & \multicolumn{5}{|c|}{500 Thousand Cubic Feet Per Day } \\
\hline Flowlines and Connections........................... . & 316.1 & 338.7 & 358.1 & 375.8 & 23,300 \\
\hline Production Package ..................................... & 173.8 & 173.8 & 185.7 & 190.5 & 8,000 \\
\hline 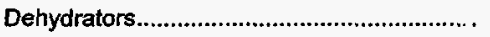 & 157.9 & 156.8 & 165.3 & 172.6 & 16,400 \\
\hline 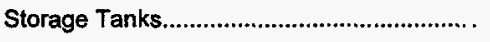 & 163.6 & 170.9 & 174.5 & 180.0 & 9,900 \\
\hline \multirow[t]{2}{*}{ 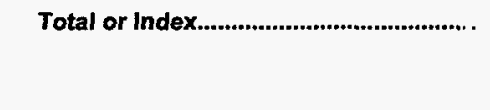 } & 200.4 & 207.1 & 217.7 & 226.8 & 57,600 \\
\hline & \multicolumn{5}{|c|}{1 Million Cubic Feet Per Day } \\
\hline Flowlines and Connections........................... . & 323.1 & 348.1 & 367.3 & 386.5 & 20,100 \\
\hline Production Package & 109.0 & 109.0 & 110.0 & 113.0 & 11,300 \\
\hline 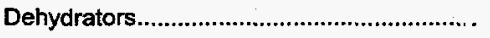 & 157.9 & 156.8 & 165.3 & 172.6 & 16,400 \\
\hline 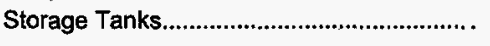 & 163.6 & 170.9 & 174.5 & 180.0 & 9,900 \\
\hline \multirow[t]{2}{*}{ Total or Index. } & 171.2 & 176.5 & 183.4 & 191.1 & 57,700 \\
\hline & \multicolumn{5}{|c|}{5 Million Cubic Feet Per Day } \\
\hline Flowlines and Connections.............................. & 301.2 & 323.8 & 342.7 & 359.8 & 59,000 \\
\hline Production Package & 129.1 & 123.6 & 120.9 & 124.5 & 13,700 \\
\hline 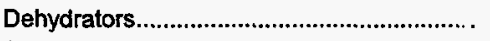 & 187.0 & 187.0 & 187.0 & 203.5 & 23,400 \\
\hline 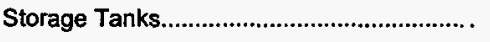 & 163.6 & 170.9 & 174.5 & 180.0 & 9,900 \\
\hline 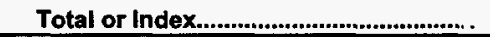 & 211.9 & 219.8 & 226.6 & 238.7 & 106,000 \\
\hline
\end{tabular}


Table 15. Direct Annual Operating Costs and Indices for Gas Production in South Texas (1 Well Producing from 2,000 Feet)

\begin{tabular}{|c|c|c|c|c|c|}
\hline \multirow{2}{*}{ Component } & \multicolumn{4}{|c|}{ Index $(1976=100)$} & \multirow{2}{*}{$\begin{array}{c}\text { 1997 } \\
\text { Cost } \\
\text { (dollars) }\end{array}$} \\
\hline & 1994 & 1995 & 1996 & 1997 & \\
\hline & \multicolumn{5}{|c|}{50 Thousand Cubic Feet Per Day } \\
\hline Direct Labor \& Overhead................................. . & 276.9 & 292.3 & 292.3 & 300.0 & 3,900 \\
\hline Fuel, Chemicals \& Disposal............................ & 300.0 & 300.0 & 300.0 & 300.0 & 600 \\
\hline 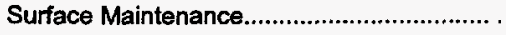 & 236.8 & 242.1 & 236.8 & 231.6 & 4,400 \\
\hline Subsurface Maintenance.................................... & 200.0 & 200.0 & 200.0 & 220.0 & 1,100 \\
\hline \multirow[t]{2}{*}{ Total or Index $x^{* *}$} & 248.7 & 256.4 & 253.8 & 256.4 & 10,000 \\
\hline & \multicolumn{5}{|c|}{250 Thousand Cubic Feet Per Day } \\
\hline Direct Labor \& Overhead................................... . & 276.9 & 292.3 & 292.3 & 300.0 & 3,900 \\
\hline Fuel, Chemicals \& Disposal............................ & 220.0 & 220.0 & 220.0 & 240.0 & 2,400 \\
\hline Surface Maintenance. & 236.8 & 242.1 & 236.8 & 231.6 & 4,400 \\
\hline 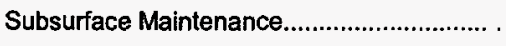 & 200.0 & 200.0 & 200.0 & 220.0 & 1,100 \\
\hline Total or Index & 240.4 & 246.8 & 244.7 & 251.1 & 11,800 \\
\hline
\end{tabular}

Table 16. Direct Annual Operating Costs and Indices for Gas Production in South Texas (1 Well Producing from 4,000 Feet)

\begin{tabular}{|c|c|c|c|c|c|}
\hline \multirow{2}{*}{ Component } & \multicolumn{4}{|c|}{ Index $(1976=100)$} & \multirow{2}{*}{$\begin{array}{c}1997^{\star} \\
\text { Cost } \\
\text { (dollars) }\end{array}$} \\
\hline & 1994 & 1995 & 1996 & 1997 & \\
\hline & \multicolumn{5}{|c|}{50 Thousand Cubic Feet Per Day } \\
\hline Direct Labor \& Overhead................................ . & 292.9 & 300.0 & 307.1 & 314.3 & 4,400 \\
\hline Fuel, Chemicals \& Disposal.......................... & 220.0 & 220.0 & 220.0 & 240.0 & 1,200 \\
\hline Surface Maintenance...................................... & 236.8 & 242.1 & 236.8 & 231.6 & 4,400 \\
\hline Subsurface Maintenance .................................... & 200.0 & 200.0 & 200.0 & 214.3 & 1,500 \\
\hline \multirow[t]{2}{*}{ 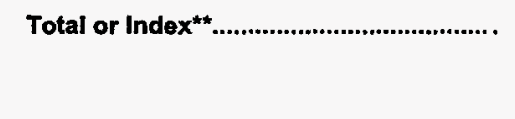 } & 246.7 & 251.1 & 251.1 & 255.6 & 11,500 \\
\hline & \multicolumn{5}{|c|}{250 Thousand Cubic Feet Per Day } \\
\hline Direct Labor \& Overhead.................................. . & 292.9 & 300.0 & 307.1 & 314.3 & 4,400 \\
\hline Fuel, Chemicals \& Disposal............................ & 220.0 & 220.0 & 220.0 & 235.0 & 4,700 \\
\hline 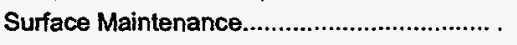 & 216.7 & 220.8 & 216.7 & 212.5 & 5,100 \\
\hline Subsurface Maintenance.................................... . & 200.0 & 200.0 & 200.0 & 214.3 & 1,500 \\
\hline Total or Index ${ }^{* *}$ & 232.3 & 235.4 & 235.4 & 241.5 & 15,700 \\
\hline
\end{tabular}


Table 17. Direct Annual Operating Costs and Indices for Gas Production in South Texas (1 Well Producing from 8,000 Feet)

\begin{tabular}{|c|c|c|c|c|c|}
\hline \multirow{2}{*}{ Component } & \multicolumn{4}{|c|}{ Index $(1976=100)$} & \multirow{2}{*}{$\begin{array}{c}1997^{\star} \\
\text { Cost } \\
\text { (dollars) }\end{array}$} \\
\hline & 1994 & 1995 & 1996 & 1997 & \\
\hline & \multicolumn{5}{|c|}{250 Thousand Cubic Feet Per Day } \\
\hline Direct Labor \& Overhead................................. . & 276.5 & 288.2 & 294.1 & 300.0 & 5,100 \\
\hline Fuel, Chemicals \& Disposal......................... . & 225.6 & 225.6 & 225.6 & 243.6 & 9,500 \\
\hline Surface Maintenance...................................... . & 210.6 & 212.8 & 210.6 & 206.4 & 9,700 \\
\hline Subsurface Maintenance................................... . & 200.0 & 208.3 & 208.3 & 216.7 & 2,600 \\
\hline \multirow[t]{2}{*}{ Total or Index ${ }^{* *}$} & 224.3 & 227.8 & 227.8 & 233.9 & 26,900 \\
\hline & \multicolumn{5}{|c|}{500 Thousand Cubic Feet Per Day } \\
\hline Direct Labor \& Overhead................................ . & 276.5 & 288.2 & 294.1 & 300.0 & 5,100 \\
\hline Fuel, Chemicals \& Disposal.......................... . & 137.5 & 137.5 & 140.0 & 137.5 & 5,500 \\
\hline Surface Maintenance...................................... . & 209.8 & 209.8 & 209.8 & 204.9 & 8,400 \\
\hline Subsurface Maintenance.................................. . & 200.0 & 208.3 & 208.3 & 216.7 & 2,600 \\
\hline \multirow[t]{2}{*}{ Total or Index } & 192.7 & 195.5 & 197.3 & 196.4 & 21,600 \\
\hline & \multicolumn{5}{|c|}{1 Million Cubic Feet Per Day } \\
\hline Direct Labor \& Overhead.............................. . & 276.5 & 288.2 & 294.1 & 300.0 & 5,100 \\
\hline Fuel, Chemicals \& Disposal........................... . & 286.5 & 284.6 & 288.5 & 292.3 & 15,200 \\
\hline Surface Maintenance.......................................... . & 206.5 & 208.7 & 208.7 & 206.5 & 9,500 \\
\hline Subsurface Maintenance................................ . & 200.0 & 208.3 & 208.3 & 216.7 & 2,600 \\
\hline Total or Index & 248.0 & 250.4 & 252.8 & 255.1 & 32,400 \\
\hline
\end{tabular}

Table 18. Direct Annual Operating Costs and indices for Gas Production in South Texas (1 Well Producing from 12,000 Feet)

\begin{tabular}{|c|c|c|c|c|c|}
\hline \multirow{2}{*}{ Component } & \multicolumn{4}{|c|}{ Index $(1976=100)$} & \multirow{2}{*}{$\begin{array}{c}\text { 1997* } \\
\text { Cost } \\
\text { (dollars) }\end{array}$} \\
\hline & 1994 & 1995 & 1996 & 1997 & \\
\hline & \multicolumn{5}{|c|}{500 Thousand Cubic Feet Per Day } \\
\hline Direct Labor \& Overhead................................. . & 285.0 & 295.0 & 300.0 & 310.0 & 6,200 \\
\hline Fuel, Chemicals \& Disposal........................... . & 217.2 & 213.8 & 217.2 & 217.2 & 6,300 \\
\hline Surface Maintenance..................................... & 219.0 & 221.4 & 221.4 & 216.7 & 9,100 \\
\hline Subsurface Maintenance................................. . & 208.3 & 216.7 & 216.7 & 229.2 & 5,500 \\
\hline \multirow[t]{2}{*}{ Total or Index } & 229.8 & 233.3 & 235.1 & 237.7 & 27,100 \\
\hline & \multicolumn{5}{|c|}{1 Million Cubic Feet Per Day } \\
\hline Direct Labor \& Overhead.................................. . & 285.0 & 295.0 & 300.0 & 310.0 & 6,200 \\
\hline Fuel, Chemicals \& Disposal.......................... . & 178.3 & 176.7 & 181.7 & 180.0 & 10,800 \\
\hline 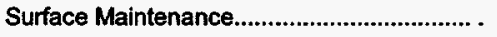 & 206.5 & 208.7 & 208.7 & 206.5 & 9,500 \\
\hline Subsurface Maintenance................................. . & 208.3 & 216.7 & 216.7 & 229.2 & 5,500 \\
\hline \multirow[t]{2}{*}{ Total or Index } & 207.4 & 210.1 & 212.8 & 214.8 & 32,000 \\
\hline & \multicolumn{5}{|c|}{5 Million Cubic Feet Per Day } \\
\hline Direct Labor \& Overhead............................... . & 285.0 & 295.0 & 300.0 & 310.0 & 6,200 \\
\hline Fuel, Chemicals \& Disposal........................... . & 163.2 & 163.2 & 177.2 & 180.7 & 10,300 \\
\hline Surface Maintenance........................................ . & 198.4 & 201.6 & 204.8 & 204.8 & 12,900 \\
\hline Subsurface Maintenance................................... . & 192.3 & 200.0 & 200.0 & 211.5 & 5,500 \\
\hline Total or Index ${ }^{\star *} \ldots \ldots \ldots \ldots$ & 195.8 & 199.4 & 206.0 & 210.2 & 34,900 \\
\hline
\end{tabular}


Table J1. Lease Equipment Costs and Indices for Gas Production in South Louisiana (1 Well Producing from 2,000 Feet)

\begin{tabular}{|c|c|c|c|c|c|}
\hline \multirow{2}{*}{ Component } & \multicolumn{4}{|c|}{ Index $(1976=100)$} & \multirow{2}{*}{$\begin{array}{c}1997^{*} \\
\text { Cost } \\
\text { (dollars) }\end{array}$} \\
\hline & 1994 & 1995 & 1996 & 1997 & \\
\hline & \multicolumn{5}{|c|}{50 Thousand Cubic Feet Per Day } \\
\hline Flowlines and Connections............................... & 242.9 & 250.0 & 257.1 & 278.6 & 3,900 \\
\hline 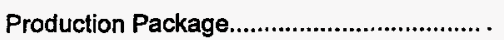 & 160.0 & 162.9 & 174.3 & 182.9 & 6,400 \\
\hline 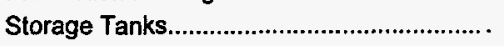 & 162.5 & 169.6 & 175.0 & 182.1 & 10,200 \\
\hline \multirow[t]{2}{*}{ 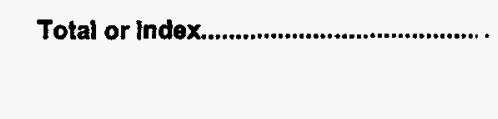 } & 172.4 & 178.1 & 185.7 & 195.2 & 20,500 \\
\hline & \multicolumn{5}{|c|}{250 Thousand Cubic Feet Per Day } \\
\hline Flowlines and Connections............................ & 242.9 & 250.0 & 257.1 & 278.6 & 3,900 \\
\hline 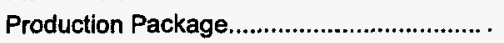 & 160.0 & 162.9 & 174.3 & 182.9 & 6,400 \\
\hline 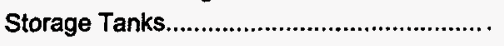 & 162.5 & 169.6 & 175.0 & 182.1 & 10,200 \\
\hline Total or Index & 172.4 & 178.1 & 185.7 & 195.2 & 20,500 \\
\hline
\end{tabular}

Table J2. Lease Equipment Costs and Indices for Gas Production in South Louisiana (1 Well Producing from 4,000 Feet)

\begin{tabular}{|c|c|c|c|c|c|}
\hline \multirow{2}{*}{ Component } & \multicolumn{4}{|c|}{ Index $(1976=100)$} & \multirow{2}{*}{$\begin{array}{c}\text { 1997* } \\
\text { Cost } \\
\text { (dollars) }\end{array}$} \\
\hline & 1994 & 1995 & 1996 & 1997 & \\
\hline & \multicolumn{5}{|c|}{50 Thousand Cubic Feet Per Day } \\
\hline Flowlines and Connections........................... . & 242.9 & 250.0 & 257.1 & 278.6 & 3,900 \\
\hline Production Package....................................... & 160.0 & 162.9 & 174.3 & 182.9 & 6,400 \\
\hline 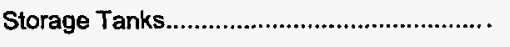 & 162.5 & 169.6 & 175.0 & 182.1 & 10,200 \\
\hline \multirow[t]{2}{*}{ 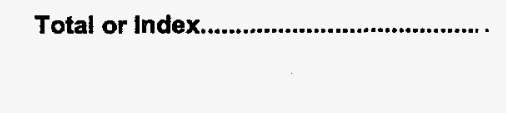 } & 172.4 & 178.1 & 185.7 & 195.2 & 220,500 \\
\hline & \multicolumn{5}{|c|}{250 Thousand Cubic Feet Per Day } \\
\hline Flowlines and Connections.......................... & 206.5 & 219.6 & 230.4 & 243.5 & 11,200 \\
\hline Production Package & 197.3 & 200.0 & 213.5 & 224.3 & 8,300 \\
\hline 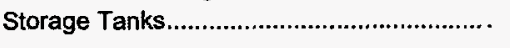 & 162.5 & 169.6 & 175.0 & 182.1 & 10,200 \\
\hline Total or index & 186.3 & 194.2 & 203.6 & 213.7 & 29,700 \\
\hline
\end{tabular}


Table J3. Lease Equipment Costs and Indices for Gas Production in South Louisiana (1 Well Producing from 8,000 Feet)

\begin{tabular}{|c|c|c|c|c|c|}
\hline \multirow{2}{*}{ Component } & \multicolumn{4}{|c|}{ Index $(1976=100)$} & \multirow{2}{*}{$\begin{array}{c}1997 \\
\text { Cost } \\
\text { (dollars) }\end{array}$} \\
\hline & 1994 & 1995 & 1996 & 1997 & \\
\hline & \multicolumn{5}{|c|}{250 Thousand Cubic Feet Per Day } \\
\hline Flowlines and Connections........................... . & 204.1 & 218.4 & 228.6 & 240.8 & 11,800 \\
\hline Production Package....................................... & 197.3 & 200.0 & 213.5 & 224.3 & 8,300 \\
\hline 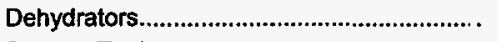 & 156.3 & 155.2 & 163.5 & 175.0 & 16,800 \\
\hline 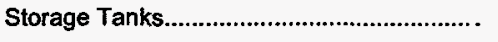 & 162.5 & 169.6 & 175.0 & 182.1 & 10,200 \\
\hline \multirow[t]{2}{*}{ 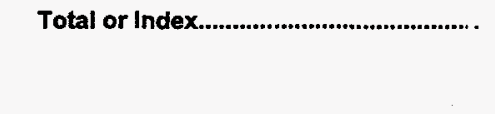 } & 173.9 & 178.6 & 187.4 & 197.9 & 47,100 \\
\hline & \multicolumn{5}{|c|}{500 Thousand Cubic Feet Per Day } \\
\hline Flowlines and Connections............................. & 202.4 & 217.1 & 229.3 & 241.5 & 9,900 \\
\hline Production Package ..................................... & 173.8 & 176.2 & 188.1 & 197.6 & 8,300 \\
\hline 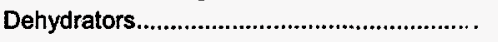 & 156.3 & 155.2 & 163.5 & 175.0 & 16,800 \\
\hline 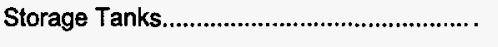 & 162.5 & 169.6 & 175.0 & 182.1 & 10,200 \\
\hline \multirow[t]{2}{*}{ 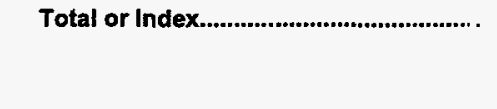 } & 168.9 & 173.2 & 182.1 & 192.3 & 45,200 \\
\hline & \multicolumn{5}{|c|}{1 Million Cubic Feet Per Day } \\
\hline Flowlines and Connections.......................... . & 323.1 & 348.1 & 367.3 & 386.5 & 20,100 \\
\hline Production Package & 109.0 & 109.0 & 110.0 & 115.0 & 11,500 \\
\hline 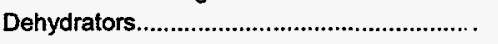 & 156.3 & 155.2 & 163.5 & 175.0 & 16,800 \\
\hline 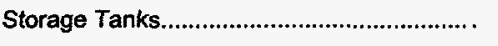 & 162.5 & 169.6 & 175.0 & 182.1 & 10,200 \\
\hline 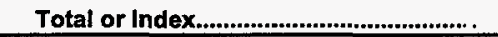 & 170.4 & 175.7 & 182.9 & 192.8 & 58,600 \\
\hline
\end{tabular}

Table J4. Lease Equipment Costs and Indices for Gas Production in South Louisiana (1 Well Producing from 12,000 Feet)

\begin{tabular}{|c|c|c|c|c|c|}
\hline \multirow{2}{*}{ Component } & \multicolumn{4}{|c|}{ Index $(1976=100)$} & \multirow{2}{*}{$\begin{array}{c}1997^{\star} \\
\text { Cost } \\
\text { (dollars) }\end{array}$} \\
\hline & 1994 & 1995 & 1996 & 1997 & \\
\hline & \multicolumn{5}{|c|}{500 Thousand Cubic Feet Per Day } \\
\hline Flowtines and Connections............................ & 316.1 & 338.7 & 358.1 & 375.8 & 23,300 \\
\hline Production Package....................................... . & 173.8 & 176.2 & 188.1 & 197.6 & 8,300 \\
\hline 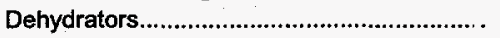 & 156.3 & 155.2 & 163.5 & 175.0 & 16,800 \\
\hline 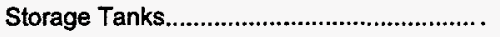 & 162.5 & 169.6 & 175.0 & 182.1 & 10,200 \\
\hline \multirow[t]{2}{*}{ 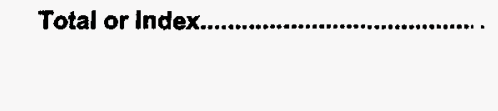 } & 199.2 & 206.3 & 217.2 & 228.9 & 58,600 \\
\hline & \multicolumn{5}{|c|}{1 Million Cubic Feet Per Day } \\
\hline Flowlines and Connections.......................... & 323.1 & 348.1 & 367.3 & 386.5 & 20,100 \\
\hline 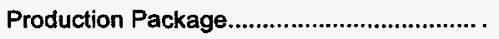 & 109.0 & 109.0 & 110.0 & 115.0 & 11,500 \\
\hline 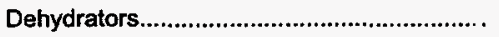 & 156.3 & 155.2 & 163.5 & 175.0 & 16,800 \\
\hline 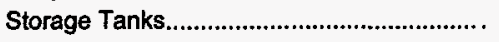 & 162.5 & 169.6 & 175.0 & 182.1 & 10,200 \\
\hline \multirow[t]{2}{*}{ 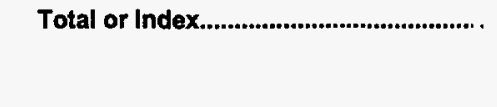 } & 170.4 & 175.7 & 182.9 & 192.8 & 58,600 \\
\hline & \multicolumn{5}{|c|}{5 Million Cubic Feet Per Day } \\
\hline Flowlines and Connections............................ . & 301.2 & 323.8 & 342.7 & 359.8 & 59,000 \\
\hline Production Package....................................... . & 129.1 & 123.6 & 121.8 & 128.2 & 14,100 \\
\hline 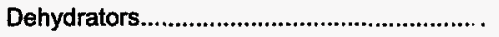 & 185.3 & 185.3 & 185.3 & 205.2 & 23,800 \\
\hline 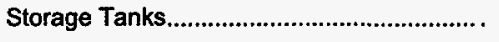 & 162.5 & 169.6 & 175.0 & 182.1 & 10,200 \\
\hline 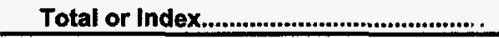 & 211.2 & 219.1 & 226.2 & 240.1 & 107,100 \\
\hline
\end{tabular}


Table J5. Lease Equipment Costs and Indices for Gas Production in South Louisiana (1 Well Producing from 16,000 Feet)

\begin{tabular}{|c|c|c|c|c|c|}
\hline \multirow{2}{*}{ Component } & \multicolumn{4}{|c|}{ Index $(1976=100)$} & \multirow{2}{*}{$\begin{array}{c}1997^{*} \\
\text { Const } \\
\text { (dollars) }\end{array}$} \\
\hline & 1994 & 1995 & 1996 & 1997 & \\
\hline . & \multicolumn{5}{|c|}{500 Thousand Cubic Feet Per Day } \\
\hline Flowlines and Connections........................ & 316.1 & 338.7 & 358.1 & 375.8 & 23,300 \\
\hline Production Package ...................................... . & 173.8 & 176.2 & 188.1 & 197.6 & 8,300 \\
\hline 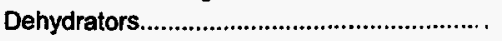 & 156.3 & 155.2 & 163.5 & 175.0 & 16,800 \\
\hline 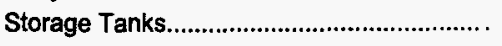 & 162.5 & 169.6 & 175.0 & 182.1 & 10,200 \\
\hline \multirow[t]{2}{*}{ 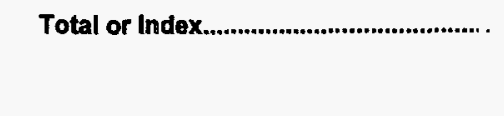 } & 199.2 & 206.3 & 217.2 & 228.9 & 58,600 \\
\hline & \multicolumn{5}{|c|}{1 Million Cubic Feet Per Day } \\
\hline Flowlines and Connections.......................... . & 323.1 & 348.1 & 367.3 & 386.5 & 20,100 \\
\hline Production Package....................................... . & 109.0 & 109.0 & 110.0 & 115.0 & 11,500 \\
\hline 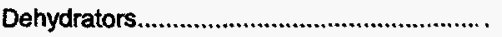 & 156.3 & 155.2 & 163.5 & 175.0 & 16,800 \\
\hline 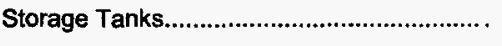 & 162.5 & 169.6 & 175.0 & 182.1 & 10,200 \\
\hline \multirow[t]{2}{*}{ Total or Index................................................ } & 170.4 & 175.7 & 182.9 & 192.8 & 58,600 \\
\hline & \multicolumn{5}{|c|}{5 Million Cubic Feet Per Day } \\
\hline Flowlines and Connections........................... & 301.2 & 323.8 & 342.7 & 359.8 & 59,000 \\
\hline Production Package.................................... & 129.1 & 123.6 & 121.8 & 128.2 & 14,100 \\
\hline 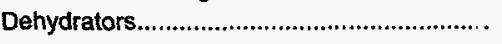 & 185.3 & 185.3 & 185.3 & 205.2 & 23,800 \\
\hline 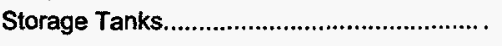 & 162.5 & 169.6 & 175.0 & 182.1 & 10,200 \\
\hline 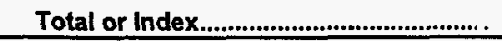 & 211.2 & 219.1 & 226.2 & 240.1 & 107,100 \\
\hline
\end{tabular}

Table J6. Direct Annual Operating Costs and Indices for Gas Production in South Louisiana (1 Well Producing from 2,000 Feet)

\begin{tabular}{|c|c|c|c|c|c|}
\hline \multirow{2}{*}{ Component } & \multicolumn{4}{|c|}{ Index $(1976=100)$} & \multirow{2}{*}{$\begin{array}{c}19197^{\star} \\
\text { Cost } \\
\text { (dollars) }\end{array}$} \\
\hline & 1994 & 1995 & 1996 & 1997 & \\
\hline & \multicolumn{5}{|c|}{50 Thousand Cubic Feet Per Day } \\
\hline Direct Labor \& Overhead................................. . & 269.2 & 284.6 & 284.6 & 307.7 & 4,000 \\
\hline 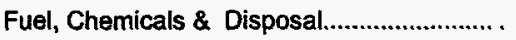 & 300.0 & 300.0 & 300.0 & 300.0 & 600 \\
\hline 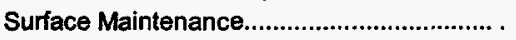 & 205.0 & 210.0 & 210.0 & 235.0 & 4,700 \\
\hline Subsurface Maintenance.................................. . & 166.7 & 166.7 & 166.7 & 166.7 & 1,000 \\
\hline \multirow[t]{2}{*}{ Total or Index $x^{* \star} \ldots$} & 224.4 & 231.7 & 231.7 & 251.2 & 10,300 \\
\hline & \multicolumn{5}{|c|}{250 Thousand Cubic Feet Per Day } \\
\hline Direct Labor \& Overhead................................. . & 269.2 & 284.6 & 284.6 & 307.7 & 4,000 \\
\hline Fuel, Chemicals \& Disposal............................ . & 220.0 & 220.0 & 220.0 & 240.0 & 2,400 \\
\hline Surface Maintenance......................................... . & 205.0 & 210.0 & 210.0 & 235.0 & 4,700 \\
\hline Subsurface Maintenance.................................. . & 166.7 & 166.7 & 166.7 & 166.7 & 1,000 \\
\hline Total or Index & 220.4 & 226.5 & 226.5 & 246.9 & 12,100 \\
\hline
\end{tabular}


Table J7. Direct Annual Operating Costs and Indices for Gas Production in South Louisiana (1 Well Producing from 4,000 Feet)

\begin{tabular}{|c|c|c|c|c|c|}
\hline \multirow{2}{*}{ Component } & \multicolumn{4}{|c|}{ Index $(1976=100)$} & \multirow{2}{*}{$\begin{array}{c}1997^{\star} \\
\text { Cost } \\
\text { (dollars) }\end{array}$} \\
\hline & 1994 & 1995 & 1996 & 1997 & \\
\hline & \multicolumn{5}{|c|}{50 Thousand Cubic Feet Per Day } \\
\hline Direct Labor \& Overhead.................................... . & 285.7 & 292.9 & 300.0 & 321.4 & 4,500 \\
\hline Fuel, Chemicals \& Disposal.......................... & 220.0 & 220.0 & 220.0 & 240.0 & 1,200 \\
\hline 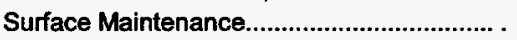 & 205.0 & 210.0 & 210.0 & 235.0 & 4,700 \\
\hline Subsurface Maintenance................................... & 175.0 & 187.5 & 187.5 & 187.5 & 1,500 \\
\hline \multirow[t]{2}{*}{ 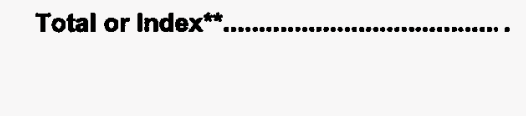 } & 225.5 & 231.9 & 234.0 & 253.2 & 11,900 \\
\hline & \multicolumn{5}{|c|}{250 Thousand Cubic Feet Per Day } \\
\hline Direct Labor \& Overhead................................ . . & 285.7 & 292.9 & 300.0 & 321.4 & 4,500 \\
\hline Fuel, Chemicals \& Disposal.......................... & 220.0 & 220.0 & 220.0 & 235.0 & 4,700 \\
\hline Surface Maintenance..................................... & 204.0 & 212.0 & 212.0 & 240.0 & 6,000 \\
\hline Subsurface Maintenance................................... . & 175.0 & 187.5 & 187.5 & 187.5 & 1,500 \\
\hline Total or Index $x^{* \star \ldots}$ & 222.4 & 228.4 & 229.9 & 249.3 & 16,700 \\
\hline
\end{tabular}

Table J8. Direct Annual Operating Costs and Indices for Gas Production in South Louisiana (1 Well Producing from 8,000 Feet)

\begin{tabular}{|c|c|c|c|c|c|}
\hline \multirow{2}{*}{ Component } & \multicolumn{4}{|c|}{ Index $(1976=100)$} & \multirow{2}{*}{$\begin{array}{c}1997^{*} \\
\text { Cost } \\
\text { (dollars) }\end{array}$} \\
\hline & 1994 & 1995 & 1996 & 1997 & \\
\hline & \multicolumn{5}{|c|}{250 Thousand Cubic Feet Per Day } \\
\hline Direct Labor \& Overhead................................ . & 270.6 & 282.4 & 288.2 & 305.9 & 5,200 \\
\hline Fuel, Chemicals \& Disposal.......................... & 225.6 & 225.6 & 225.6 & 243.6 & 9,500 \\
\hline Surface Maintenance....................................... . & 196.0 & 200.0 & 202.0 & 226.0 & 11,300 \\
\hline Subsurface Maintenance................................ . & 191.7 & 200.0 & 200.0 & 200.0 & 2,400 \\
\hline \multirow[t]{2}{*}{ Total or Index } & 216.1 & 220.3 & 222.0 & 240.7 & 28,400 \\
\hline & \multicolumn{5}{|c|}{500 Thousand Cubic Feet Per Day } \\
\hline Direct Labor \& Overhead................................ . & 270.6 & 282.4 & 288.2 & 305.9 & 5,200 \\
\hline Fuel, Chemicals \& Disposal......................... . & 211.5 & 211.5 & 215.4 & 242.3 & 6,300 \\
\hline Surface Maintenance....................................... & 198.0 & 202.0 & 204.1 & 228.6 & 11,200 \\
\hline Subsurface Maintenance............................... . & 191.7 & 200.0 & 200.0 & 200.0 & 2,400 \\
\hline \multirow[t]{2}{*}{ Total or Index ${ }^{* *}$} & 212.5 & 217.3 & 220.2 & 241.3 & 25,100 \\
\hline & \multicolumn{5}{|c|}{1 Million Cubic Feet Per Day } \\
\hline Direct Labor \& Overhead............................. . & 270.6 & 282.4 & 288.2 & 305.9 & 5,200 \\
\hline Fuel, Chemicals \& Disposal.......................... . & 213.0 & 213.0 & 218.5 & 244.4 & 13,200 \\
\hline Surface Maintenance...................................... . & 195.8 & 200.0 & 204.2 & 227.1 & 10,900 \\
\hline Subsurface Maintenance................................ . & 191.7 & 200.0 & 200.0 & 200.0 & 2,400 \\
\hline Total or Index & 212.2 & 216.0 & 220.6 & 242.0 & 31,700 \\
\hline
\end{tabular}


Table J9. Direct Annual Operating Costs and Indices for Gas Production in South Louislana (1 Well Producing from 12,000 Feet)

\begin{tabular}{|c|c|c|c|c|c|}
\hline \multirow{2}{*}{ Component } & \multicolumn{4}{|c|}{ Index $(1976=100)$} & \multirow{2}{*}{$\begin{array}{c}1997^{\star} \\
\text { Cost } \\
\text { (dollars) }\end{array}$} \\
\hline & 1994 & 1995 & 1996 & 1997 & \\
\hline & \multicolumn{5}{|c|}{500 Thousand Cuble Feet Per Day } \\
\hline Direct Labor \& Overhead....................................... . & 280.0 & 290.0 & 295.0 & 315.0 & 6,300 \\
\hline Fuel, Chemicals \& Disposal.......................... . & 206.7 & 206.7 & 213.3 & 233.3 & 7,000 \\
\hline 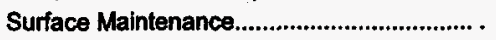 & 206.8 & 211.4 & 213.6 & 238.6 & 10,500 \\
\hline Subsurface Maintenance................................... . & 187.5 & 191.7 & 191.7 & 195.8 & 4,700 \\
\hline \multirow[t]{2}{*}{ Total or Index } & 215.3 & 219.5 & 222.9 & 241.5 & 28,500 \\
\hline & \multicolumn{5}{|c|}{1 Million Cubic Foet Per Day } \\
\hline Direct Labor \& Overhead................................. . & 280.0 & 290.0 & 295.0 & 315.0 & 6,300 \\
\hline Fuel, Chemicals \& Disposal............................... & 209.8 & 209.8 & 216.4 & 239.3 & 14,600 \\
\hline Surface Maintenance........................................... & 195.8 & 200.0 & 204.2 & 227.1 & 10,900 \\
\hline Subsurface Maintenance ................................... . & 204.2 & 212.5 & 212.5 & 216.7 & 5,200 \\
\hline \multirow[t]{2}{*}{ Total or Index $=\ldots$} & 213.7 & 217.6 & 222.2 & 241.8 & 37,1000 \\
\hline & \multicolumn{5}{|c|}{5 Million Cubic Foet Per Day } \\
\hline 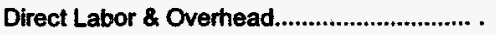 & 280.0 & 290.0 & 295.0 & 315.0 & 6,300 \\
\hline Fuel, Chemicals \& Disposal............................. & 178.0 & 168.3 & 190.2 & 195.1 & 8,000 \\
\hline Surface Maintenance.......................................... . & 205.3 & 212.3 & 217.5 & 238.6 & 13,600 \\
\hline Subsurface Maintenance ...................................... & 188.5 & 196.2 & 196.2 & 200.0 & 5,200 \\
\hline Total or Index $x^{n+\ldots}$ & 204.8 & 207.6 & 216.7 & 229.9 & 33,100 \\
\hline
\end{tabular}

Table J10. Direct Annual Operating Costs and Indices for Gas Production in South Louisiana (1 Well Producing from 16,000 Feet)

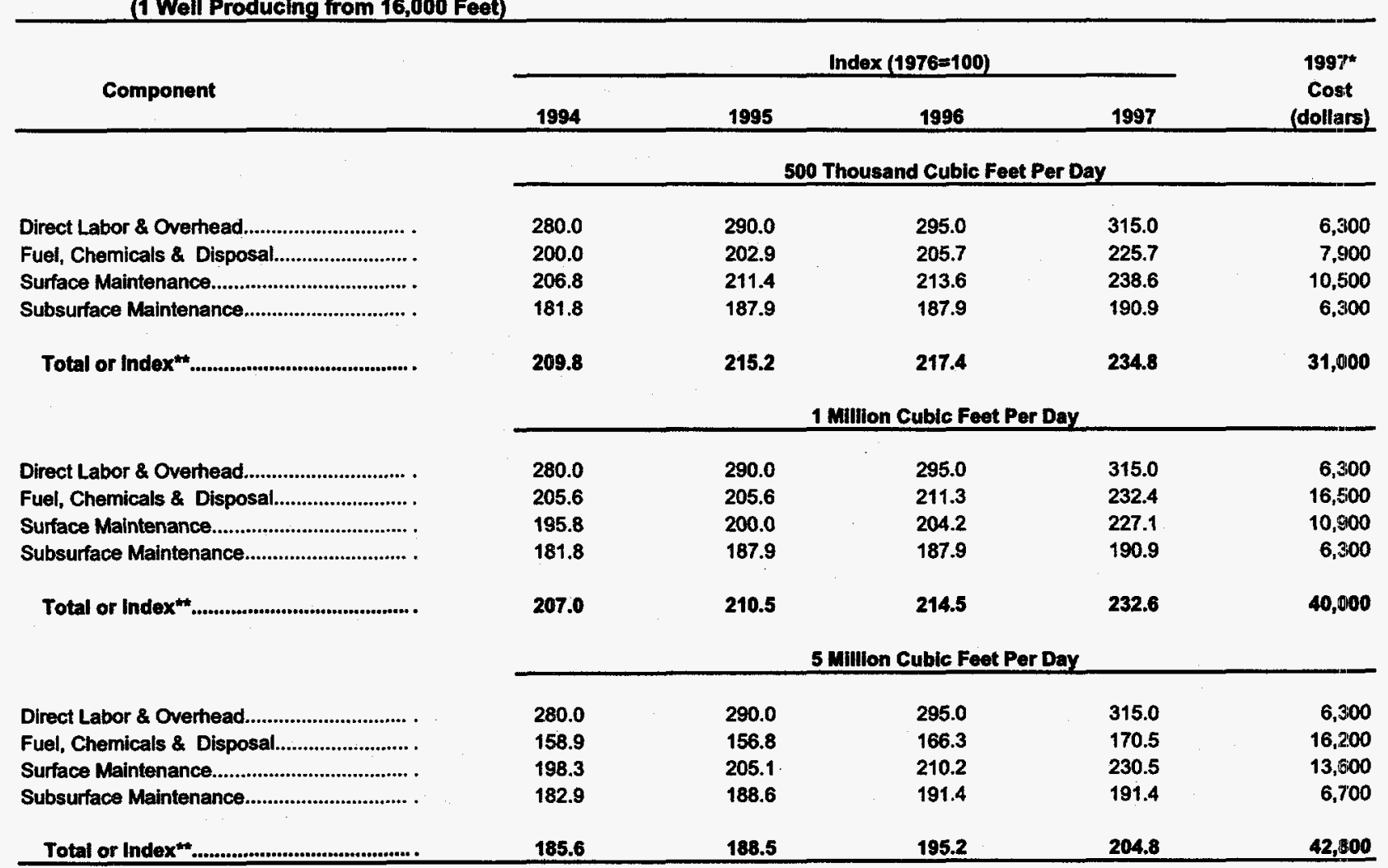


Table K1. Lease Equipment Costs and Indices for Gas Production in North Louisiana (1 Well Producing from 2,000 Feet)

\begin{tabular}{|c|c|c|c|c|c|}
\hline \multirow{2}{*}{ Component } & \multicolumn{4}{|c|}{ Index $(1976=100)$} & \multirow{2}{*}{$\begin{array}{c}1997^{\star} \\
\text { Cost } \\
\text { (dollars) }\end{array}$} \\
\hline & 1994 & 1995 & 1996 & 1997 & \\
\hline & \multicolumn{5}{|c|}{50 Thousand Cubic Feet Per Day } \\
\hline Flowlines and Connections......................... & 242.9 & 250.0 & 257.1 & 278.6 & 3,900 \\
\hline Production Package ...................................... & 160.0 & 162.9 & 174.3 & 182.9 & 6,400 \\
\hline Storage Tanks................................................ & 162.5 & 169.6 & 175.0 & 182.1 & 10,200 \\
\hline 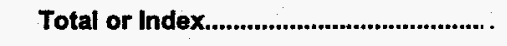 & 172.4 & 178.1 & 185.7 & 195.2 & 20,500 \\
\hline & \multicolumn{5}{|c|}{250 Thousand Cubic Feet Per Day } \\
\hline Flowlines and Connections........................ . & 242.9 & 250.0 & 257.1 & 278.6 & 3,900 \\
\hline Production Package.................................... . & 160.0 & 162.9 & 174.3 & 182.9 & 6,400 \\
\hline Storage Tanks................................................. & 162.5 & 169.6 & 175.0 & 182.1 & 10,200 \\
\hline 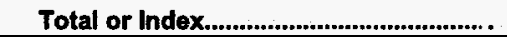 & 172.4 & 178.1 & 185.7 & 195.2 & 20,500 \\
\hline
\end{tabular}

Table K2. Lease Equipment Costs and Indices for Gas Production in North Louisiana (1 Well Producing from 4,000 Feet)

\begin{tabular}{|c|c|c|c|c|c|}
\hline \multirow{2}{*}{ Component } & \multicolumn{4}{|c|}{ Index $(1976=100)$} & \multirow{2}{*}{$\begin{array}{c}1997^{\star} \\
\text { Cost } \\
\text { (dollars) }\end{array}$} \\
\hline & 1994 & 1995 & 1996 & 1997 & \\
\hline & \multicolumn{5}{|c|}{250 Thousand Cubic Feet Per Day } \\
\hline Flowlines and Connections............................ . & 206.5 & 219.6 & 230.4 & 243.5 & 11,200 \\
\hline 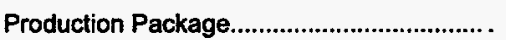 & 208.1 & 213.5 & 218.9 & 229.7 & 8,500 \\
\hline 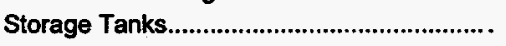 & 162.5 & 169.6 & 175.0 & 182.1 & 10,200 \\
\hline \multirow[t]{2}{*}{ 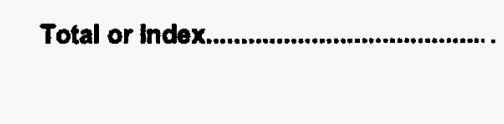 } & 189.2 & 197.8 & 205.0 & 215.1 & 29,900 \\
\hline & \multicolumn{5}{|c|}{500 Thousand Cubic Feet Per Day } \\
\hline Flowlines and Connections............................. & 283.3 & 300.0 & 316.7 & 333.3 & 2,000 \\
\hline 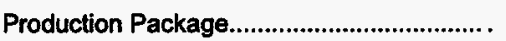 & 173.8 & 176.2 & 188.1 & 197.6 & 8,300 \\
\hline 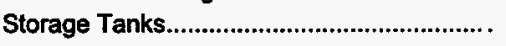 & 162.5 & 169.6 & 175.0 & 182.1 & 10,200 \\
\hline Total or Index & 174.0 & 179.8 & 188.5 & 197.1 & 20,500 \\
\hline
\end{tabular}


Table K3. Lease Equipment Costs and Indlces for Gas Production in North Louisiana (1 Well Producing from 8,000 Feet)

\begin{tabular}{|c|c|c|c|c|c|}
\hline \multirow{2}{*}{ Component } & \multicolumn{4}{|c|}{ Index $(1976=100)$} & \multirow{2}{*}{$\begin{array}{c}1997^{*} \\
\text { Cost } \\
\text { (dlollars) }\end{array}$} \\
\hline & 1994 & 1995 & 1996 & 1997 & \\
\hline & \multicolumn{5}{|c|}{250 Thousand Cubic Feet Por Day } \\
\hline Flowlines and Connections............................ . . & 204.1 & 218.4 & 228.6 & 240.8 & 11,800 \\
\hline Production Package........................................ . & 191.9 & 194.6 & 200.0 & 210.8 & 7,800 \\
\hline 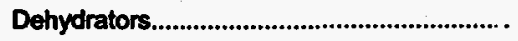 & 156.3 & 155.2 & 163.5 & 175.0 & 16,800 \\
\hline 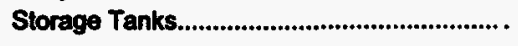 & 162.5 & 169.6 & 175.0 & 182.1 & 10,200 \\
\hline \multirow[t]{2}{*}{ 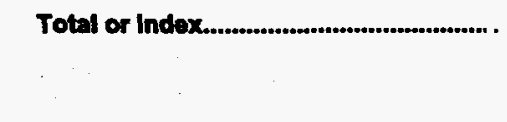 } & 173.1 & 177.7 & 185.3 & 195.8 & 46,600 \\
\hline & \multicolumn{5}{|c|}{500 Thousand Cubic Feet Per Day } \\
\hline Flowlines and Connections............................ . & 202.4 & 217.1 & 229.3 & 241.5 & 9,900 \\
\hline 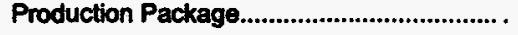 & 173.8 & 176.2 & 188.1 & 197.6 & 8,300 \\
\hline 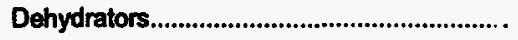 & 156.3 & 155.2 & 163.5 & 175.0 & 16,800 \\
\hline 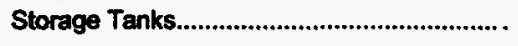 & 162.5 & 169.6 & 175.0 & 182.1 & 10,200 \\
\hline Total or Index & 168.9 & 173.2 & 182.1 & 192.3 & 45,200 \\
\hline
\end{tabular}

Table K4. Lease Equipment Costs and Indices for Gas Production in North Louisiana (1 Well Producing from 12,000 Feet)

\begin{tabular}{|c|c|c|c|c|c|}
\hline \multirow{2}{*}{ Component } & \multicolumn{4}{|c|}{ Index $(1976=100)$} & \multirow{2}{*}{$\begin{array}{c}1997^{\star} \\
\text { Cost } \\
\text { (clollars) }\end{array}$} \\
\hline & 1994 & 1995 & 1996 & 1997 & \\
\hline & \multicolumn{5}{|c|}{500 Thousand Cubic Feet Per Day } \\
\hline Flowlines and Connections............................ . & 316.1 & 338.7 & 358.1 & 375.8 & 23,300 \\
\hline 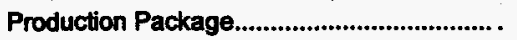 & 173.8 & 176.2 & 188.1 & 197.6 & 8,300 \\
\hline 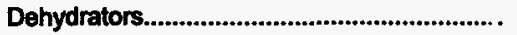 & 156.3 & 155.2 & 163.5 & 175.0 & 16,800 \\
\hline 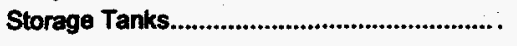 & 162.5 & 169.6 & 175.0 & 182.1 & 10,200 \\
\hline \multirow{2}{*}{ 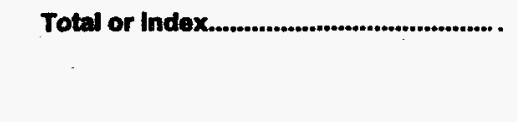 } & 199.2 & 206.3 & 217.2 & 228.9 & 58,600 \\
\hline & \multicolumn{5}{|c|}{1 Million Cubic Feet Per Day } \\
\hline Flowlines and Connections.............................. . & 323.1 & 348.1 & 367.3 & 386.5 & 20,100 \\
\hline 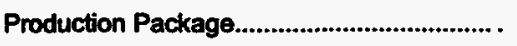 & 109.0 & 109.0 & 110.0 & 115.0 & 11,500 \\
\hline 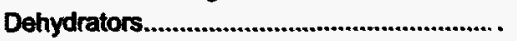 & 156.3 & 155.2 & 163.5 & 175.0 & 16,800 \\
\hline 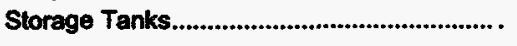 & 162.5 & 169.6 & 175.0 & 182.1 & 10,200 \\
\hline Total or Index & 170.4 & 175.7 & 182.9 & 192.8 & 58,600 \\
\hline
\end{tabular}


Table K5. Lease Equipment Costs and Indices for Gas Production in North Louisiana (1 Well Producing from 16,000 Feet)

\begin{tabular}{|c|c|c|c|c|c|}
\hline \multirow{2}{*}{ Component } & \multicolumn{4}{|c|}{ Index $(1976=100)$} & \multirow{2}{*}{$\begin{array}{c}1997^{*} \\
\text { Cost } \\
\text { (dollars) }\end{array}$} \\
\hline & 1994 & 1995 & 1996 & 1997 & \\
\hline & \multicolumn{5}{|c|}{1 Million Cubic Foet Por Day } \\
\hline Flowlines and Connections................................ & 323.1 & 348.1 & 367.3 & 386.5 & 20,100 \\
\hline 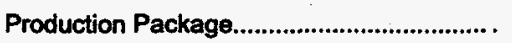 & 109.0 & 109.0 & 110.0 & 115.0 & 11,500 \\
\hline 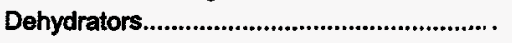 & 156.3 & 155.2 & 163.5 & 175.0 & 16,800 \\
\hline 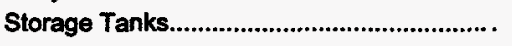 & 162.5 & 169.6 & 175.0 & 182.1 & 10,200 \\
\hline \multirow[t]{2}{*}{ 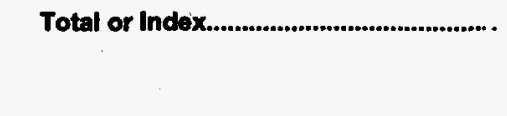 } & 170.4 & 175.7 & 182.9 & 192.8 & 58,600 \\
\hline & \multicolumn{5}{|c|}{5 Million Cubic Feet Per Day } \\
\hline Flowlines and Connections.......................... . & 301.2 & 323.8 & 342.7 & 359.8 & 59,000 \\
\hline Production Package........................................ & 129.1 & 123.6 & 121.8 & 128.2 & 14,100 \\
\hline 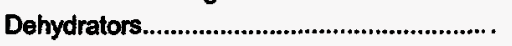 & 185.3 & 185.3 & 185.3 & 205.2 & 23,800 \\
\hline 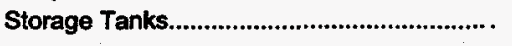 & 162.5 & 169.6 & 175.0 & 182.1 & 10,200 \\
\hline \multirow[t]{2}{*}{ 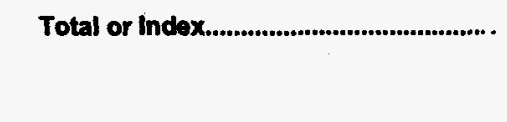 } & 211.2 & 219.1 & 226.2 & 240.1 & 107,100 \\
\hline & \multicolumn{5}{|c|}{10 Million Cubic Feet Per Day } \\
\hline Flowlines and Connections............................. . & 301.2 & 323.8 & 342.7 & 359.8 & 59,000 \\
\hline 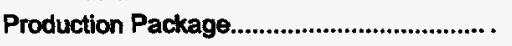 & 129.1 & 123.6 & 121.8 & 128.2 & 14,100 \\
\hline 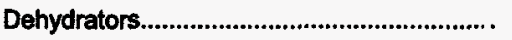 & 208.4 & 208.7 & 211.4 & 222.4 & 58,500 \\
\hline 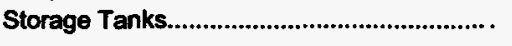 & 162.5 & 169.6 & 175.0 & 182.1 & 10,200 \\
\hline 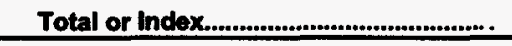 & 215.0 & 221.1 & 227.7 & 239.1 & 141,800 \\
\hline
\end{tabular}

Table K6. Direct Annual Operating Costs and Indices for Gas Production in North Louisiana (1 Well Producing from 2,000 Feet)

\begin{tabular}{|c|c|c|c|c|c|}
\hline \multirow{2}{*}{ Component } & \multicolumn{4}{|c|}{ Index $(1976=100)$} & \multirow{2}{*}{$\begin{array}{c}1997^{*} \\
\text { Cost } \\
\text { (dollars) }\end{array}$} \\
\hline & 1994 & 1995 & 1996 & 1997 & \\
\hline & \multicolumn{5}{|c|}{50 Thousand Cubic Feet Per Day } \\
\hline Direct Labor \& Overhead.................................. . & 269.2 & 284.6 & 284.6 & 307.7 & 4,000 \\
\hline Fuel, Chemicals \& Disposal.......................... . & 250.0 & 250.0 & 250.0 & 300.0 & 600 \\
\hline Surface Maintenance......................................... , & 205.0 & 210.0 & 210.0 & 235.0 & 4,700 \\
\hline Subsurface Maintenance..................................... . & 166.7 & 166.7 & 166.7 & 166.7 & 1,000 \\
\hline \multirow[t]{2}{*}{ Total or Index ${ }^{\text {th }}$} & 222.0 & 229.3 & 229.3 & 251.2 & 10,300 \\
\hline & \multicolumn{5}{|c|}{250 Thousand Cuble Feet Per Day } \\
\hline Direct Labor \& Overhead................................... . . & 269.2 & 284.6 & 284.6 & 307.7 & 4,000 \\
\hline Fuel, Chemicals \& Disposal........................... . & 210.0 & 210.0 & 210.0 & 220.0 & 2,200 \\
\hline Surface Maintenance....................................... . & 205.0 & 210.0 & 210.0 & 235.0 & 4,700 \\
\hline Subsurface Maintenance.................................... . & 166.7 & 166.7 & 166.7 & 166.7 & 1,000 \\
\hline Total or Index $x^{* \ldots}$ & 218.4 & 224.5 & 224.5 & 242.9 & 11,900 \\
\hline
\end{tabular}


Table K7. Direct Annual Operating Costs and Indices for Gas Production in North Louisiana (1 Well Producing from 4,000 Feet)

\begin{tabular}{|c|c|c|c|c|c|}
\hline \multirow{2}{*}{ Component } & \multicolumn{4}{|c|}{ Index $(1976=100)$} & \multirow{2}{*}{$\begin{array}{c}1997^{*} \\
\text { Cost } \\
\text { (diollars) }\end{array}$} \\
\hline & 1994 & 1995 & 1996 & 1997 & \\
\hline & \multicolumn{5}{|c|}{250 Thousand Cubic Feet Per Day } \\
\hline Direct Labor \& Overhead.................................... . & 285.7 & 292.9 & 300.0 & 321.4 & 4,500 \\
\hline Fuel, Chemicals \& Disposal............................ & 205.0 & 205.0 & 205.0 & 220.0 & 4,400 \\
\hline Surface Maintenance...................................... . & 204.0 & 208.0 & 216.0 & 240.0 & 6,000 \\
\hline Subsurface Maintenance................................. & 175.0 & 187.5 & 187.5 & 187.5 & 1,500 \\
\hline \multirow[t]{2}{*}{ Total or Index ${ }^{* *} \ldots$} & 217.9 & 222.4 & 226.9 & 244.8 & 16,400 \\
\hline & \multicolumn{5}{|c|}{500 Thousand Cubic Feet Per Day } \\
\hline Direct Labor \& Overhead................................. . & 285.7 & 292.9 & 300.0 & 321.4 & 4,500 \\
\hline Fuel, Chemicals \& Disposal........................ . & 200.0 & 200.0 & 204.2 & 229.2 & 5,500 \\
\hline Surface Maintenance....................................... . & 204.0 & 208.0 & 208.0 & 236.0 & 5,900 \\
\hline Subsurface Maintenance................................... . & 175.0 & 187.5 & 187.5 & 187.5 & 1,500 \\
\hline 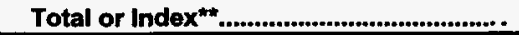 & 215.5 & 219.7 & 222.5 & 245.1 & 17,400 \\
\hline
\end{tabular}

Table K8. Direct Annual Operating Costs and Indices for Gas Production in North Louisiana (1 Well Producing from 8,000 Feet)

\begin{tabular}{|c|c|c|c|c|c|}
\hline \multirow{2}{*}{ Component } & \multicolumn{4}{|c|}{ Index $(1976=100)$} & \multirow{2}{*}{$\begin{array}{c}1997^{\star} \\
\text { Cost } \\
\text { (dollars) }\end{array}$} \\
\hline & 1994 & 1995 & 1996 & 1997 & \\
\hline & \multicolumn{5}{|c|}{250 Thousand Cubic Feet Per Day } \\
\hline Direct Labor \& Overhead............................... . & 270.6 & 282.4 & 288.2 & 305.9 & 5,200 \\
\hline Fuel, Chemicals \& Disposal......................... . & 212.8 & 212.8 & 212.8 & 228.2 & 8,900 \\
\hline Surface Maintenance.................................... & 196.0 & 200.0 & 202.0 & 228.0 & 11,400 \\
\hline Subsurface Maintenance............................... & 216.7 & 225.0 & 225.0 & 225.0 & 2,700 \\
\hline \multirow[t]{2}{*}{ Total or lndex } & 214.4 & 218.6 & 220.3 & 239.0 & 28,200 \\
\hline & \multicolumn{5}{|c|}{500 Thousand Cubic Feet Per Day } \\
\hline Direct Labor \& Overhead................................ . & 270.6 & 282.4 & 288.2 & 305.9 & 5,200 \\
\hline Fuel, Chemicals \& Disposal........................ . & 188.5 & 188.5 & 192.3 & 215.4 & 5,600 \\
\hline Surface Maintenance.................................... . & 198.0 & 202.0 & 204.1 & 228.6 & 11,200 \\
\hline Subsurface Maintenance................................ . & 216.7 & 225.0 & 225.0 & 225.0 & 2,700 \\
\hline Total or Index & 209.6 & 214.4 & 217.3 & 237.5 & 24,700 \\
\hline
\end{tabular}


Table K9. Direct Annual Operating Costs and Indices for Gas Production in North Louisiana (1 Well Producing from 12,000 Feet)

\begin{tabular}{|c|c|c|c|c|c|}
\hline \multirow{2}{*}{ Component } & \multicolumn{4}{|c|}{ Index $(1976=100)$} & \multirow{2}{*}{$\begin{array}{c}1997^{*} \\
\text { Cost } \\
\text { (dollars) }\end{array}$} \\
\hline & 1994 & 1995 & 1996 & 1997 & \\
\hline & \multicolumn{5}{|c|}{500 Thousand Cubic Feet Per Day } \\
\hline Direct Labor \& Overhead................................. . & 280.0 & 290.0 & 295.0 & 315.0 & 6,300 \\
\hline Fuel, Chemicals \& Disposal.......................... . & 200.0 & 203.3 & 206.7 & 226.7 & 6,800 \\
\hline Surface Maintenance..................................... . & 206.8 & 211.4 & 213.6 & 238.6 & 10,500 \\
\hline Subsurface Maintenance............................... & 145.8 & 154.2 & 154.2 & 158.3 & 3,800 \\
\hline \multirow[t]{2}{*}{ Total or Index } & 205.1 & 211.0 & 213.6 & 232.2 & 27,400 \\
\hline & \multicolumn{5}{|c|}{1 Million Cubic Feet Per Day } \\
\hline Direct Labor \& Overhead............................... . & 280.0 & 290.0 & 295.0 & 315.0 & 6,300 \\
\hline Fuel, Chemicals \& Disposal......................... . & 206.6 & 206.6 & 213.1 & 236.1 & 14,400 \\
\hline Surface Maintenance.................................. . & 195.8 & 200.0 & 204.2 & 227.1 & 10,900 \\
\hline Subsurface Maintenance................................ . & 145.8 & 154.2 & 154.2 & 158.3 & 3,800 \\
\hline Total or Index ${ }^{* k} \ldots \ldots \ldots \ldots \ldots \ldots \ldots \ldots$ & 203.3 & 207.2 & 211.8 & 231.4 & 35,400 \\
\hline
\end{tabular}

Table K10. Direct Annual Operating Costs and Indices for Gas Production in North Louisiana (1 Well Producing from 16,000 Feet)

\begin{tabular}{|c|c|c|c|c|c|}
\hline \multirow{2}{*}{ Component } & \multicolumn{4}{|c|}{ Index $(1976=100)$} & \multirow{2}{*}{$\begin{array}{c}1997^{\star} \\
\text { Cost } \\
\text { (dollars) }\end{array}$} \\
\hline & 1994 & 1995 & 1996 & 1997 & \\
\hline & \multicolumn{5}{|c|}{1 Million Cubic Feet Per Day } \\
\hline Direct Labor \& Overhead.................................. . & 280.0 & 290.0 & 295.0 & 315.0 & 6,300 \\
\hline Fuel, Chemicals \& Disposal.......................... . & 202.8 & 202.8 & 208.5 & 229.6 & 16,300 \\
\hline Surface Maintenance.................................... & 193.8 & 197.9 & 202.1 & 225.0 & 10,800 \\
\hline Subsurface Maintenance.................................. . & 181.8 & 187.9 & 187.9 & 190.9 & 6,300 \\
\hline \multirow[t]{2}{*}{ Total or Index ${ }^{* *}$} & 205.2 & 208.7 & 212.8 & 230.8 & 39,700 \\
\hline & \multicolumn{5}{|c|}{5 Million Cubic Feot Per Day } \\
\hline Direct Labor \& Overhead............................... . & 280.0 & 290.0 & 295.0 & 315.0 & 6,300 \\
\hline Fuel, Chemicals \& Disposal.......................... . & 160.4 & 157.3 & 168.8 & 172.9 & 16,600 \\
\hline Surface Maintenance.................................... . & 196.6 & 203.4 & 208.5 & 230.5 & 13,600 \\
\hline Subsurface Maintenance.................................. . & 182.9 & 188.6 & 191.4 & 191.4 & 6,700 \\
\hline \multirow[t]{2}{*}{ 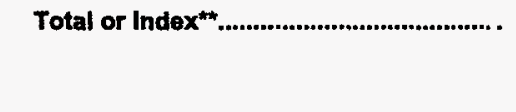 } & 185.7 & 188.1 & 195.7 & 205.7 & 43,200 \\
\hline & \multicolumn{5}{|c|}{10 Million Cubic Feet Per Day } \\
\hline Direct Labor \& Overhead................................ . & 280.0 & 290.0 & 295.0 & 315.0 & 6,300 \\
\hline Fuel, Chemicals \& Disposal........................... & 155.8 & 152.1 & 163.8 & 168.1 & 27,400 \\
\hline Surface Maintenance....................................... . & 207.2 & 213.0 & 217.4 & 237.7 & 16,400 \\
\hline Subsurface Maintenance................................. . & 182.9 & 188.6 & 191.4 & 191.4 & 6,700 \\
\hline Total or Index & 180.1 & 180.8 & 189.2 & 197.9 & 56,800 \\
\hline
\end{tabular}


Table L1. Lease Equipment Costs and Indices for Gas Production in the Mid-Continent (1 Well Producing from 2,000 Feet)

\begin{tabular}{|c|c|c|c|c|c|}
\hline \multirow{2}{*}{ Component } & \multicolumn{4}{|c|}{ Index $(1976=100)$} & \multirow{2}{*}{$\begin{array}{c}1997^{*} \\
\text { Cos:t } \\
\text { (dollars) }\end{array}$} \\
\hline & 1994 & 1995 & 1996 & 1997 & \\
\hline & \multicolumn{5}{|c|}{50 Thousand Cubic Foet Per Day } \\
\hline Flowlines and Connections.............................. & 269.2 & 276.9 & 284.6 & 300.0 & 3,900 \\
\hline 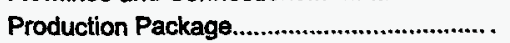 & 164.7 & 164.7 & 176.5 & 185.3 & 6,300 \\
\hline 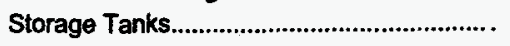 & 170.4 & 177.8 & 183.3 & 190.7 & 10,300 \\
\hline \multirow[t]{2}{*}{ 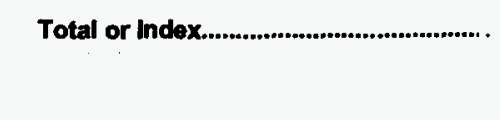 } & 181.2 & 186.1 & 194.1 & 203.0 & 20,500 \\
\hline & \multicolumn{5}{|c|}{250 Thousand Cubic Feot Per Day } \\
\hline Flowlines and Connections............................ & 269.2 & 276.9 & 284.6 & 300.0 & 3,900 \\
\hline 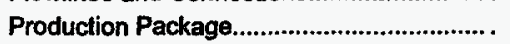 & 185.0 & 185.0 & 195.0 & 205.0 & $\mathbf{8 , 2 0 0}$ \\
\hline 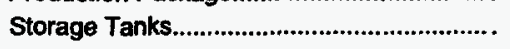 & 170.4 & 177.8 & 183.3 & 190.7 & 10,300 \\
\hline Total or Index & 187.9 & 192.5 & 200.0 & 209.3 & 22,400 \\
\hline
\end{tabular}

Table L2. Lease Equipment Costs and Indices for Gas Production in the Mid-Continent (1 Woll Producing from 4,000 Feet)

\begin{tabular}{|c|c|c|c|c|c|}
\hline \multirow{2}{*}{ Component } & \multicolumn{4}{|c|}{ Index $(1976=100)$} & \multirow{2}{*}{$\begin{array}{c}\text { 1997 } \\
\text { Cost } \\
\text { (dollairs) }\end{array}$} \\
\hline & 1994 & 1995 & 1996 & 1997 & \\
\hline & \multicolumn{5}{|c|}{50 Thousand Cubic Feet Per Day } \\
\hline Flowines and Connections............................ . & 269.2 & 276.9 & 284.6 & 300.0 & 3,900 \\
\hline Production Package....................................... . & 164.7 & 164.7 & 176.5 & 185.3 & 6,300 \\
\hline Storage Tanks.............................................. & 170.4 & 177.8 & 183.3 & 190.7 & 10,300 \\
\hline \multirow[t]{2}{*}{ 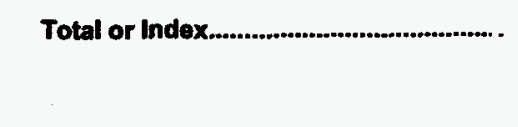 } & 181.2 & 186.1 & 194.1 & 203.0 & 20,500 \\
\hline & \multicolumn{5}{|c|}{250 Thousand Cubic Feet Per Day } \\
\hline Flowlines and Connections........................... & 213.3 & 226.7 & 235.6 & 248.9 & 11,200 \\
\hline Production Package...................................... . & 202.9 & 208.6 & 208.6 & 220.0 & 7,700 \\
\hline 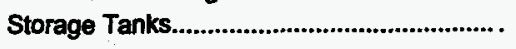 & 170.4 & 177.8 & 183.3 & 190.7 & 10,300 \\
\hline \multirow[t]{2}{*}{ Total or Index........................................... } & 193.3 & 202.2 & 207.5 & 217.9 & 29,200 \\
\hline & \multicolumn{5}{|c|}{500 Thousand Cubic Feet Per Day } \\
\hline Flowlines and Connections........................... . & 205.3 & 221.1 & 231.6 & 244.7 & 9,300 \\
\hline Production Package...................................... . & 202.9 & 208.6 & 208.6 & 220.0 & 7,7010 \\
\hline 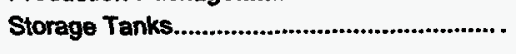 & 170.4 & 177.8 & 183.3 & 190.7 & 10,300 \\
\hline 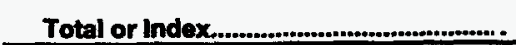 & 189.8 & 199.2 & 204.7 & 215.0 & 27,300 \\
\hline
\end{tabular}


Table L3. Lease Equipment Costs and Indices for Gas Production in the Mid-Continent (1 Well Producing from 8,000 Feet)

\begin{tabular}{|c|c|c|c|c|c|}
\hline \multirow{2}{*}{ Component } & \multicolumn{4}{|c|}{ Index (1976-100) } & \multirow{2}{*}{$\begin{array}{c}1997^{*} \\
\text { Cost } \\
\text { (dollars) }\end{array}$} \\
\hline & 1994 & 1995 & 1996 & 1997 & \\
\hline & \multicolumn{5}{|c|}{250 Thousand Cubic Feet Per Day } \\
\hline Flowlines and Connections............................ . & 210.4 & 222.9 & 233.3 & 245.8 & 11,800 \\
\hline 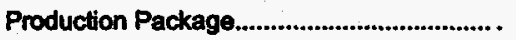 & 202.9 & 208.6 & 208.6 & 220.0 & 7,700 \\
\hline 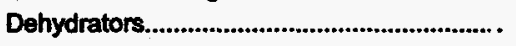 & 163.4 & 161.3 & 171.0 & 181.7 & 16,900 \\
\hline 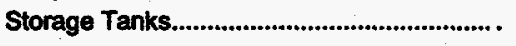 & 170.4 & 177.8 & 183.3 & 190.7 & 10,300 \\
\hline \multirow[t]{2}{*}{ 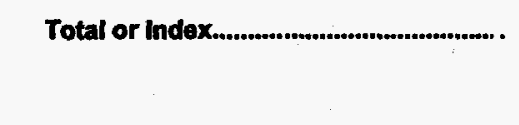 } & 180.9 & 185.2 & 192.6 & 203.0 & 46,700 \\
\hline & \multicolumn{5}{|c|}{500 Thousand Cubic Feet Per Day } \\
\hline Flowlines and Connections............................. . & 202.4 & 217.1 & 229.3 & 241.5 & 9,900 \\
\hline 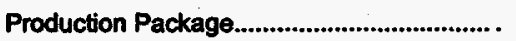 & 185.0 & 185.0 & 185.0 & 185.0 & 7,400 \\
\hline 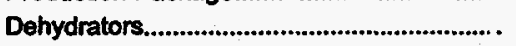 & 163.4 & 161.3 & 171.0 & 181.7 & 16,900 \\
\hline 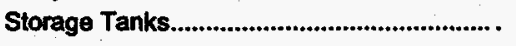 & 170.4 & 177.8 & 183.3 & 190.7 & 10,300 \\
\hline 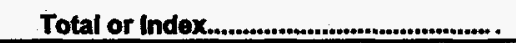 & 175.9 & 179.4 & 186.8 & 195.2 & 44,500 \\
\hline
\end{tabular}

Table L4. Lease Equipment Costs and Indices for Gas Production in the Mid-Continent (1 Woll Producing from 12,000 Foet)

\begin{tabular}{|c|c|c|c|c|c|}
\hline \multirow{2}{*}{ Component } & \multicolumn{4}{|c|}{ Index $(1976=100)$} & \multirow{2}{*}{$\begin{array}{c}1997^{\star} \\
\text { Cost } \\
\text { (dollars) }\end{array}$} \\
\hline & 1994 & 1995 & 1996 & 1997 & \\
\hline & \multicolumn{5}{|c|}{250 Thousand Cubic Feet Per Day } \\
\hline Flowlines and Connections.......................... . & 308.7 & 330.4 & 347.8 & 365.2 & 25,200 \\
\hline Production Package......................................... . & 185.0 & 185.0 & 195.0 & 205.0 & 8,200 \\
\hline 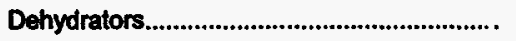 & 163.4 & 161.3 & 171.0 & 181.7 & 16,900 \\
\hline 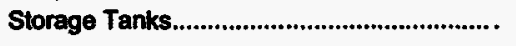 & 170.4 & 177.8 & 183.3 & 190.7 & 10,300 \\
\hline \multirow[t]{2}{*}{ 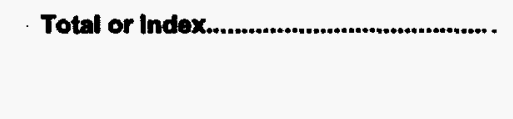 } & 207.4 & 214.1 & 225.0 & 236.7 & 60,600 \\
\hline & \multicolumn{5}{|c|}{500 Thousand Cubic Fest Per Day } \\
\hline Flowlines and Connections............................ . & 316.1 & 338.7 & 358.1 & 375.8 & 23,300 \\
\hline 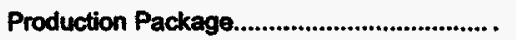 & 185.0 & 185.0 & 195.0 & 205.0 & 8,200 \\
\hline 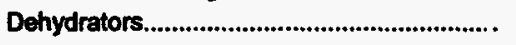 & 163.4 & 161.3 & 171.0 & 181.7 & 16,900 \\
\hline 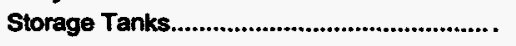 & 170.4 & 177.8 & 183.3 & 190.7 & 10,300 \\
\hline \multirow[t]{2}{*}{ 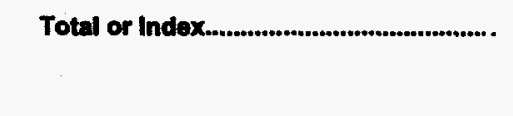 } & 206.4 & 212.9 & 224.1 & 235.7 & 58,700 \\
\hline & \multicolumn{5}{|c|}{1 million Cubic Feet Per Day } \\
\hline Flowlines and Connections........................... . & 323.1 & 348.1 & 367.3 & 386.5 & 20,100 \\
\hline Production Package...................................... . & 110.1 & 110.1 & 110.1 & 115.2 & 11,400 \\
\hline 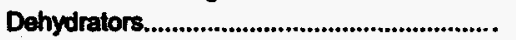 & 163.4 & 161.3 & 171.0 & 181.7 & 16,900 \\
\hline 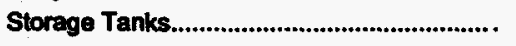 & 170.4 & 177.8 & 183.3 & 190.7 & 10,300 \\
\hline 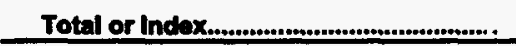 & 174.8 & 179.9 & 187.2 & 197.0 & 58,700 \\
\hline
\end{tabular}


Table L5. Lease Equipment Costs and Indices for Gas Production in the Mid-Continent (1 Well Producing from 16,000 Feet)

\begin{tabular}{|c|c|c|c|c|c|}
\hline \multirow{2}{*}{ Component } & \multicolumn{4}{|c|}{ Index $(1976=100)$} & \multirow{2}{*}{$\begin{array}{c}1997^{\text {rtk }} \\
\text { Cost } \\
\text { (dollars) }\end{array}$} \\
\hline & 1994 & 1995 & 1996 & 1997 & \\
\hline & \multicolumn{5}{|c|}{500 Thousand Cuble Foet Per Day } \\
\hline Flowlines and Connections............................. & 323.1 & 348.1 & 367.3 & 386.5 & 20,100 \\
\hline Production Package ......................................... & 110.1 & 110.1 & 110.1 & 115.2 & 11,400 \\
\hline 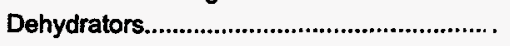 & 163.4 & 161.3 & 171.0 & 181.7 & 16,900 \\
\hline 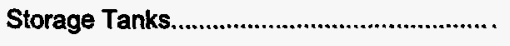 & 170.4 & 177.8 & 183.3 & 190.7 & 10,300 \\
\hline \multirow[t]{2}{*}{ Total or Index } & 174.8 & 179.9 & 187.2 & 197.0 & 58,700 \\
\hline & \multicolumn{5}{|c|}{1 Million Cubic Feet Per Day } \\
\hline Flowlines and Connections.............................. . & 323.1 & 348.1 & 367.3 & 386.5 & 20,100 \\
\hline Production Package & 110.1 & 110.1 & 110.1 & 115.2 & 11,400 \\
\hline 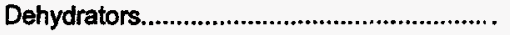 & 163.4 & 161.3 & 171.0 & 181.7 & 16,900 \\
\hline 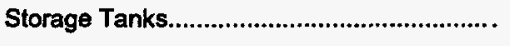 & 170.4 & 177.8 & 183.3 & 190.7 & 10,300 \\
\hline \multirow[t]{2}{*}{ 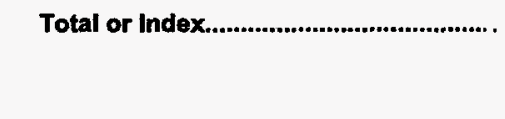 } & 174.8 & 179.9 & 187.2 & 197.0 & 58,700 \\
\hline & \multicolumn{5}{|c|}{5 Million Cubic Feet Per Day } \\
\hline Flowlines and Connections............................ . & 301.2 & 323.8 & 342.7 & 359.8 & 59,000 \\
\hline 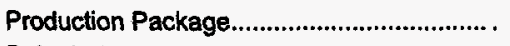 & 131.5 & 125.0 & 123.1 & 127.8 & 13,800 \\
\hline 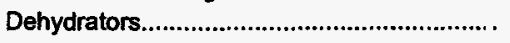 & 192.0 & 192.0 & 192.0 & 211.5 & 23,900 \\
\hline 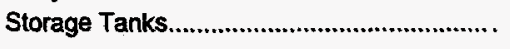 & 170.4 & 177.8 & 183.3 & 190.7 & 10,300 \\
\hline Total or Index & 215.3 & 223.0 & 230.3 & 243.7 & 107,000 \\
\hline
\end{tabular}

Table L6. Direct Annual Operating Costs and Indices for Gas Production in the Mid-Continent 1 Well Producing from 2,000 Feet)

\begin{tabular}{|c|c|c|c|c|c|}
\hline \multirow{2}{*}{ Component } & \multicolumn{4}{|c|}{ Index $(1976=100)$} & \multirow{2}{*}{$\begin{array}{c}\text { 1997' } \\
\text { Cost } \\
\text { (dollars) }\end{array}$} \\
\hline & 1994 & 1995 & 1996 & 1997 & \\
\hline & \multicolumn{5}{|c|}{50 Thousand Cubic Feet Per Day } \\
\hline Direct Labor \& Overhead............................... . & 258.3 & 275.0 & 283.3 & 300.0 & 3,600 \\
\hline Fuel, Chemicals \& Disposal......................... . & 300.0 & 300.0 & 300.0 & 300.0 & 600 \\
\hline 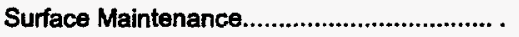 & 300.0 & 300.0 & 300.0 & 333.3 & 5,000 \\
\hline Subsurface Maintenance............................... . & 200.0 & 200.0 & 220.0 & 220.0 & 1,100 \\
\hline \multirow[t]{2}{*}{ Total or Index } & 270.6 & 276.5 & 282.4 & 302.9 & 10,3010 \\
\hline & \multicolumn{5}{|c|}{250 Thousand Cubic Feet Per Day } \\
\hline Direct Labor \& Overhead............................... . & 258.3 & 275.0 & 283.3 & 300.0 & 3,600 \\
\hline Fuel, Chemicals \& Disposal......................... . & 220.0 & 220.0 & 220.0 & 240.0 & 2,400 \\
\hline Surface Maintenance....................................... . & 300.0 & 300.0 & 295.0 & 330.0 & 6,600 \\
\hline Subsurface Maintenance................................. & 200.0 & 200.0 & 220.0 & 220.0 & 1,100 \\
\hline Total or Index & 261.7 & 266.0 & 268.1 & 291.5 & 13,700 \\
\hline
\end{tabular}


Table L7. Direct Annual Operating Costs and Indices for Gas Production in the Mid-Continent (1 Well Producing from 4,000 Feet)

\begin{tabular}{|c|c|c|c|c|c|}
\hline \multirow{2}{*}{ Component } & \multicolumn{4}{|c|}{ Index $(1976=100)$} & \multirow{2}{*}{$\begin{array}{c}\text { 1997* } \\
\text { Cost } \\
\text { (dollars) }\end{array}$} \\
\hline & 1994 & 1995 & 1996 & 1997 & \\
\hline & \multicolumn{5}{|c|}{50 Thousand Cubic Feet Per Day } \\
\hline Direct Labor \& Overhead................................ . & 250.0 & 264.3 & 271.4 & 285.7 & 4,000 \\
\hline Fuel, Chemicals \& Disposal.......................... & 220.0 & 220.0 & 220.0 & 240.0 & 1,200 \\
\hline Surface Maintenance...................................... & 300.0 & 300.0 & 300.0 & 333.3 & 5,000 \\
\hline Subsurface Maintenance................................ . & 175.0 & 175.0 & 187.5 & 187.5 & 1,500 \\
\hline \multirow[t]{2}{*}{ Total or Index } & 250.0 & 254.8 & 259.5 & 278.6 & 11,700 \\
\hline & \multicolumn{5}{|c|}{250 Thousand Cubic Feet Per Day } \\
\hline Direct Labor \& Overhead................................... . & 250.0 & 264.3 & 271.4 & 285.7 & 4,000 \\
\hline Fuel, Chemicals \& Disposal.......................... . & 220.0 & 220.0 & 220.0 & 235.0 & 4,700 \\
\hline Surface Maintenance..................................... & 291.7 & 295.8 & 295.8 & 329.2 & 7,900 \\
\hline Subsurface Maintenance.............................. . & 175.0 & 175.0 & 187.5 & 187.5 & 1,500 \\
\hline \multirow[t]{2}{*}{ Total or index $x^{* *}$} & 247.0 & 251.5 & 254.5 & 274.2 & 18,100 \\
\hline & \multicolumn{5}{|c|}{ 500 Thousand Cubic Feet Per Day } \\
\hline Direct Labor \& Overhead................................ . & 250.0 & 264.3 & 271.4 & 285.7 & 4,000 \\
\hline Fuel, Chemicals \& Disposal.......................... . & 278.9 & 278.9 & 278.9 & 305.3 & 5,800 \\
\hline Surface Maintenance....................................... . & 294.7 & 294.7 & 294.7 & 326.3 & 6,200 \\
\hline Subsurface Maintenance.............................. . & 175.0 & 175.0 & 187.5 & 187.5 & 1,500 \\
\hline Total or Index & 263.3 & 266.7 & 270.0 & 291.7 & 17,500 \\
\hline
\end{tabular}

Table L8. Direct Annual Operating Costs and Indices for Gas Production in the Mid-Continent (1 Well Producing from 8,000 Feet)

\begin{tabular}{|c|c|c|c|c|c|}
\hline \multirow{2}{*}{ Component } & \multicolumn{4}{|c|}{ Index $(1976=100)$} & \multirow{2}{*}{$\begin{array}{c}1997^{\star} \\
\text { Cost } \\
\text { (dollars) }\end{array}$} \\
\hline & 1994 & 1995 & 1996 & 1997 & \\
\hline & \multicolumn{5}{|c|}{250 Thousand Cubic Feet Per Day } \\
\hline Direct Labor \& Overhead................................. . & 256.3 & 268.8 & 275.0 & 293.8 & 4,700 \\
\hline Fuel, Chemicals \& Disposal........................... . & 228.2 & 225.6 & 228.2 & 243.6 & $\mathbf{9 , 5 0 0}$ \\
\hline Surface Maintenance.................................. & 274.4 & 276.9 & 276.9 & 305.1 & 11,900 \\
\hline Subsurface Maintenance................................. . & 175.0 & 183.3 & 183.3 & 191.7 & 2,300 \\
\hline \multirow[t]{2}{*}{ Total or Index } & 243.4 & 246.2 & 248.1 & 267.9 & 28,400 \\
\hline & \multicolumn{5}{|c|}{500 Thousand Cubic Feet Per Day } \\
\hline Direct Labor \& Overhead................................ . & 256.3 & 268.8 & 275.0 & 293.8 & 4,700 \\
\hline Fuel, Chemicals \& Disposal......................... . & 281.0 & 281.0 & 281.0 & 309.5 & 6,500 \\
\hline Surface Maintenance...................................... . & 273.5 & 273.5 & 273.5 & 300.0 & 10,200 \\
\hline Subsurface Maintenance................................ & 175.0 & 183.3 & 183.3 & 191.7 & 2,300 \\
\hline Total or Index & 257.8 & 261.4 & 262.7 & 285.5 & 23,700 \\
\hline
\end{tabular}


Table L9. Direct Annual Operating Costs and Indices for Gas Production in the Mid-Continent (1 Well Producing from 12,000 Feet)

\begin{tabular}{|c|c|c|c|c|c|}
\hline \multirow{2}{*}{ Component } & \multicolumn{4}{|c|}{ Index $(1976=100)$} & \multirow{2}{*}{$\begin{array}{c}1997^{*} \\
\text { Cost } \\
\text { (dollars) }\end{array}$} \\
\hline & 1994 & 1995 & 1996 & 1997 & \\
\hline & \multicolumn{5}{|c|}{250 Thousand Cubic Feet Per Day } \\
\hline Direct Labor \& Overhead.............................. . & 257.9 & 273.7 & 278.8 & 300.0 & 5,700 \\
\hline Fuel, Chemicals \& Disposal.......................... . & 222.0 & 222.0 & 222.0 & 238.0 & 11,900 \\
\hline Surface Maintenance...................................... & 282.5 & 285.0 & 285.0 & 315.0 & 12,600 \\
\hline Subsurface Maintenance.............................. . & 175.0 & 175.0 & 179.2 & 183.3 & 4,400 \\
\hline \multirow[t]{2}{*}{ Total or Index } & 236.8 & 239.8 & 241.4 & 260.2 & 34,600 \\
\hline & \multicolumn{5}{|c|}{500 Thousand Cubic Feet Per Day } \\
\hline Direct Labor \& Overhead.............................. . & 257.9 & 273.7 & 278.9 & 300.0 & 5,700 \\
\hline Fuel, Chemicals \& Disposal......................... . & 264.0 & 264.0 & 264.0 & 288.0 & 7,200 \\
\hline Surface Maintenance ...................................... & 282.9 & 285.7 & 285.7 & 314.3 & 11,000 \\
\hline Subsurface Maintenance................................ . & 175.0 & 175.0 & 179.2 & 183.3 & 4,400 \\
\hline \multirow[t]{2}{*}{ Total or Index } & 248.5 & 252.4 & 254.4 & 274.8 & 28,300 \\
\hline & \multicolumn{5}{|c|}{1 Million Cubic Feet Per Day } \\
\hline Direct Labor \& Overhead............................... . & 257.9 & 273.7 & 278.9 & 300.0 & 5,700 \\
\hline Fuel, Chemicals \& Disposal........................... . & 268.6 & 266.7 & 272.5 & 298.0 & 15,200 \\
\hline 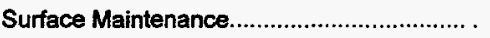 & 261.5 & 261.5 & 264.1 & 289.7 & 11,300 \\
\hline Subsurface Maintenance................................ & 175.0 & 175.0 & 179.2 & 183.3 & 4,400 \\
\hline Total or Index ${ }^{\star *}, \ldots \ldots \ldots \ldots$ & 248.1 & 249.6 & 254.1 & 275.2 & 36,600 \\
\hline
\end{tabular}

Table L10. Direct Annual Operating Costs and Indices for Gas Production in the Mid-Continent (1 Well Producing from 16,000 Feet)

\begin{tabular}{|c|c|c|c|c|c|}
\hline \multirow{2}{*}{ Component } & \multicolumn{4}{|c|}{ Index $(1976=100)$} & \multirow{2}{*}{$\begin{array}{c}\text { 1997* } \\
\text { Cost } \\
\text { (dollars) }\end{array}$} \\
\hline & 1994 & 1995 & 1996 & 1997 & \\
\hline & \multicolumn{5}{|c|}{500 Thousand Cubic Feet Per Day } \\
\hline Direct Labor \& Overhead.................................. . & 257.9 & 273.7 & 278.9 & 300.0 & 5,700 \\
\hline 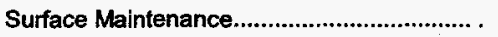 & 261.5 & 261.5 & 264.1 & 289.7 & 11,300 \\
\hline Subsurface Maintenance ................................. . & 175.9 & 175.9 & 182.8 & 186.2 & 5,400 \\
\hline \multirow[t]{2}{*}{ Total or Index } & 237.3 & 239.0 & 244.1 & 262.7 & 31,000 \\
\hline & \multicolumn{5}{|c|}{1 Million Cubic Feet Per Day } \\
\hline Fuel, Chemicals \& Disposal......................... . & 252.5 & 252.5 & 257.4 & 280.3 & 17,100 \\
\hline Surface Maintenance....................................... . & 261.5 & 261.5 & 264.1 & 289.7 & 11,300 \\
\hline Subsurface Maintenance................................. . & 175.9 & 175.9 & 182.8 & 186.2 & 5,400 \\
\hline \multirow[t]{2}{*}{ Total or Index } & 240.5 & 242.6 & 247.3 & 266.9 & 39,500 \\
\hline & \multicolumn{5}{|c|}{5 Million Cubic Feet Per Day } \\
\hline Direct Labor \& Overhead................................. . & 257.9 & 273.7 & 278.9 & 300.0 & 5,700 \\
\hline Fuel, Chemicals \& Disposal........................... . & 152.6 & 152.6 & 163.9 & 169.1 & 16,400 \\
\hline Surface Maintenance .......................................... . & 269.4 & 273.5 & 277.6 & 304.1 & 14,900 \\
\hline Subsurface Maintenance............................... . & 122.6 & 122.6 & 129.0 & 132.3 & 4,100 \\
\hline Total or Index & 187.2 & 189.8 & 198.0 & 209.7 & 41,100 \\
\hline
\end{tabular}


Table M1. Lease Equipment Costs and Indices for Gas Production in the Rocky Mountains (1 Well Producing from 2,000 Feet)

\begin{tabular}{|c|c|c|c|c|c|}
\hline \multirow{2}{*}{ Component } & \multicolumn{4}{|c|}{ Index $(1976=100)$} & \multirow{2}{*}{$\begin{array}{c}1997^{\star} \\
\text { Cost } \\
\text { (dollars) }\end{array}$} \\
\hline & 1994 & 1995 & 1996 & 1997 & \\
\hline & \multicolumn{5}{|c|}{50 Thousand Cuble Feet Per Day } \\
\hline Flowlines and Connections............................ . & 250.0 & 257.1 & 264.3 & 278.6 & 3,900 \\
\hline Production Package...................................... & 176.2 & 176.2 & 185.7 & 195.2 & 8,200 \\
\hline Storage Tanks............................................. & 160.7 & 167.9 & 173.2 & 178.6 & 10,000 \\
\hline \multirow[t]{2}{*}{ Total or Index.......................................... . } & 177.7 & 182.1 & 189.3 & 197.3 & 22,100 \\
\hline & \multicolumn{5}{|c|}{250 Thousand Cubic Feet Per Day } \\
\hline Flowlines and Connections........................... . & 250.0 & 257.1 & 264.3 & 278.6 & 3,900 \\
\hline Production Package...................................... . & 176.2 & 176.2 & 185.7 & 195.2 & 8,200 \\
\hline 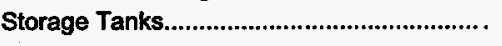 & 160.7 & 167.9 & 173.2 & 178.6 & 10,000 \\
\hline 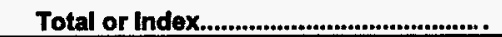 & 177.7 & 182.1 & 189.3 & 197.3 & 22,100 \\
\hline
\end{tabular}

Table M2. Lease Equipment Costs and Indices for Gas Production in the Rocky Mountains (1 Well Producing from 4,000 Feet)

\begin{tabular}{|c|c|c|c|c|c|}
\hline \multirow{2}{*}{ Component } & \multicolumn{4}{|c|}{ Index $(1976=100)$} & \multirow{2}{*}{$\begin{array}{c}1997^{*} \\
\text { Cost } \\
\text { (dollars) }\end{array}$} \\
\hline & 1994 & 1995 & 1996 & 1997 & \\
\hline & \multicolumn{5}{|c|}{50 Thousand Cubic Feet Per Day } \\
\hline Flowlines and Connections............................. & 250.0 & 257.1 & 264.3 & 278.6 & 3,900 \\
\hline Production Package ...................................... & 176.2 & 176.2 & 185.7 & 195.2 & 8,200 \\
\hline 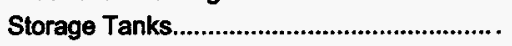 & 160.7 & 167.9 & 173.2 & 178.6 & 10,000 \\
\hline \multirow[t]{2}{*}{ Total or Index. } & 177.7 & 182.1 & 189.3 & 197.3 & 22,100 \\
\hline & \multicolumn{5}{|c|}{250 Thousand Cubic Feet Per Day } \\
\hline Flowlines and Connections........................... . & 208.7 & 221.7 & 230.4 & 243.5 & 11,200 \\
\hline 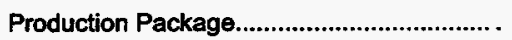 & 191.9 & 194.6 & 197.3 & 208.1 & 7,700 \\
\hline 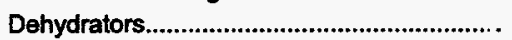 & 157.3 & 156.3 & 164.6 & 175.0 & 16,800 \\
\hline 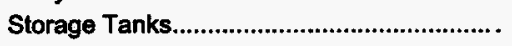 & 160.7 & 167.9 & 173.2 & 178.6 & 10,000 \\
\hline \multirow[t]{2}{*}{ 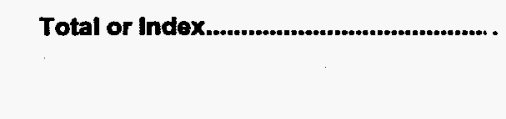 } & 173.6 & 177.9 & 184.7 & 194.5 & 45,700 \\
\hline & \multicolumn{5}{|c|}{500 Thousand Cubic Feet Per Day } \\
\hline Flowlines and Connections........................... . & 185.3 & 200.0 & 208.8 & 220.6 & 7,500 \\
\hline Production Package......................................... & 109.0 & 109.0 & 110.0 & 114.0 & 11,400 \\
\hline 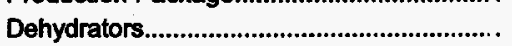 & 157.3 & 156.3 & 164.6 & 175.0 & 16,800 \\
\hline 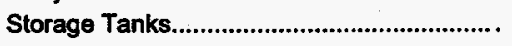 & 160.7 & 167.9 & 173.2 & 178.6 & 10,000 \\
\hline 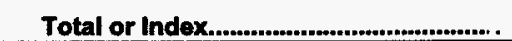 & 144.4 & 147.2 & 152.4 & 159.8 & 45,700 \\
\hline
\end{tabular}


Table M3. Lease Equipment Costs and Indices for Gas Production in the Rocky Mountains (1 Well Producing from 8,000 Feet)

\begin{tabular}{|c|c|c|c|c|c|}
\hline \multirow{2}{*}{ Component } & \multicolumn{4}{|c|}{ Index $(1976=100)$} & \multirow{2}{*}{$\begin{array}{c}1907^{*} \\
\text { Coot } \\
\text { (dollare) }\end{array}$} \\
\hline & 1994 & 1995 & 1996 & 1997 & \\
\hline & \multicolumn{5}{|c|}{250 Thousand Cuble Foet Per Day } \\
\hline Flowlines and Connections.............................. . & 193.2 & 202.3 & 213.6 & 225.0 & 9,900 \\
\hline Production Package............................................ & 109.0 & 109.0 & 110.0 & 114.0 & 11,400 \\
\hline 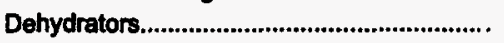 & 157.3 & 156.3 & 164.6 & 175.0 & 16,800 \\
\hline 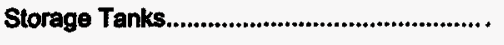 & 160.7 & 167.9 & 173.2 & 178.6 & 10,000 \\
\hline \multirow[t]{2}{*}{ 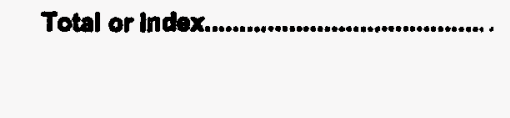 } & 147.0 & 149.3 & 155.1 & 162.5 & 48,100 \\
\hline & \multicolumn{5}{|c|}{500 Thousand Cublc Feat Per Day } \\
\hline Flowlines and Connections.............................. . & 186.1 & 200.0 & 211.1 & 222.2 & 8,000 \\
\hline 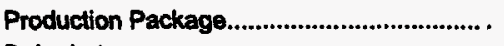 & 109.0 & 109.0 & 110.0 & 114.0 & 11,400 \\
\hline 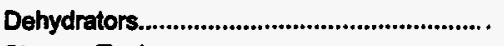 & 157.3 & 156.3 & 164.6 & 175.0 & 16,800 \\
\hline 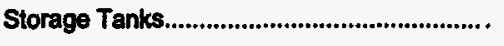 & 160.7 & 167.9 & 173.2 & 178.6 & 10,000 \\
\hline 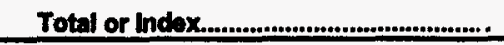 & 144.8 & 147.6 & 153.1 & 160.4 & 46,200 \\
\hline
\end{tabular}

Table M4. Lease Equipment Costs and Indices for Gas Production in the Rocky Mountains (1 Well Producing from 12,000 Feot)

\begin{tabular}{|c|c|c|c|c|c|}
\hline \multirow{2}{*}{ Component } & \multicolumn{4}{|c|}{ Index (1976=100) } & \multirow{2}{*}{$\begin{array}{c}1997 \\
\text { Cost } \\
\text { (dollars) }\end{array}$} \\
\hline & 1994 & 1995 & 1996 & 1997 & \\
\hline & \multicolumn{5}{|c|}{250 Thousand Cubic Feet Per Day } \\
\hline Flowlines and Connections.......................... . & 310.0 & 330.0 & 348.3 & 366.7 & 32,000 \\
\hline Production Package....................................... . & 109.0 & 109.0 & 110.0 & 114.0 & 11,400 \\
\hline Dehydrators...................................................... . & 157.3 & 156.3 & 164.6 & 175.0 & 16,800 \\
\hline Storage Tanks................................................ & 160.7 & 167.9 & 173.2 & 178.6 & 10,000 \\
\hline \multirow[t]{2}{*}{ Total or Index........................................ } & 171.8 & 176.6 & 184.0 & 192.9 & 00,200 \\
\hline & \multicolumn{5}{|c|}{500 Thousand Cuble Feet Per Day } \\
\hline Flowines and Connections........................... . & 323.1 & 348.1 & 367.3 & 386.5 & 20,100 \\
\hline Production Package & 109.0 & 109.0 & 110.0 & 114.0 & 11,400 \\
\hline 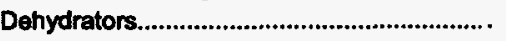 & 157.3 & 156.3 & 164.6 & 175.0 & 16,800 \\
\hline 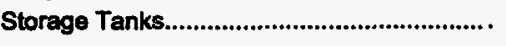 & 160.7 & 167.9 & 173.2 & 178.6 & $\because 0,000$ \\
\hline \multirow[t]{2}{*}{ Total or Index........................................... . } & 170.4 & 175.7 & 182.9 & 191.8 & 38,300 \\
\hline & \multicolumn{5}{|c|}{1 Mttlion Cubic Feet Per Day } \\
\hline Flowtines and Connections.............................. . & 323.1 & 348.1 & 367.3 & 386.5 & 20,100 \\
\hline Production Package & 109.0 & 109.0 & 110.0 & 114.0 & 11,400 \\
\hline Dehydrators.................................................... & 157.3 & 156.3 & 164.6 & 175.0 & 16,800 \\
\hline 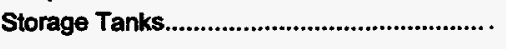 & 160.7 & 167.9 & 173.2 & 178.6 & 10,000 \\
\hline 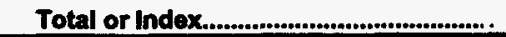 & 170.4 & 175.7 & 182.9 & 191.8 & 58,300 \\
\hline
\end{tabular}


Table M5. Direct Annual Operating Costs and Indlces for Gas Production In the Rocky Mountains (1 Well Producing from 2,000 Feet)

\begin{tabular}{|c|c|c|c|c|c|}
\hline \multirow{2}{*}{ Component } & \multicolumn{4}{|c|}{ Index $(1976=100)$} & \multirow{2}{*}{$\begin{array}{c}1997^{\star} \\
\text { Cost } \\
\text { (dollers) }\end{array}$} \\
\hline & 1994 & 1995 & 1996 & 1997 & \\
\hline & \multicolumn{5}{|c|}{50 Thousand Cubje Feet Per Day } \\
\hline Direct Labor \& Overhead.................................. . & 261.5 & 269.2 & 276.9 & 292.3 & 3,800 \\
\hline Fuel, Chemicals \& Disposal.............................. . & 300.0 & 300.0 & 300.0 & 300.0 & 600 \\
\hline Surface Maintenance....................................... . & 214.8 & 214.8 & 211.1 & 233.3 & 6,300 \\
\hline Subsurface Maintenance................................... . & 133.3 & 133.3 & 150.0 & 150.0 & 900 \\
\hline \multirow[t]{2}{*}{ Total or Index $x^{*+\ldots}$} & 220.8 & 222.9 & 225.0 & 241.7 & 11,600 \\
\hline & \multicolumn{5}{|c|}{250 Thousand Cuble Feet Per Day } \\
\hline Direct Labor \& Overhead.................................. . & 261.5 & 269.2 & 276.9 & 292.3 & 3,800 \\
\hline Fuel, Chemicals \& Disposal........................... & 220.0 & 220.0 & 220.0 & 240.0 & 2,400 \\
\hline Surface Maintenance........................................... . & 214.8 & 214.8 & 211.1 & 233.3 & 6,300 \\
\hline Subsurface Maintenance................................ . & 133.3 & 133.3 & 150.0 & 150.0 & $\mathbf{9 0 0}$ \\
\hline Total or Index & 217.9 & 219.6 & 221.4 & 239.3 & 13,400 \\
\hline
\end{tabular}

Table M6. Direct Annual Operating Costs and Indices for Gas Production in the Rocky Mountains (1 Well Producing from 4,000 Feot)

\begin{tabular}{|c|c|c|c|c|c|}
\hline \multirow{2}{*}{ Component } & \multicolumn{4}{|c|}{ Index $(1976=100)$} & \multirow{2}{*}{$\begin{array}{c}\text { 1997 } \\
\text { Cost } \\
\text { (dollars) }\end{array}$} \\
\hline & 1994 & 1995 & 1996 & 1997 & \\
\hline & \multicolumn{5}{|c|}{50 Thousand Cubic Feet Per Day } \\
\hline Direct Labor \& Overhead..................................... . & 253.3 & 266.7 & 273.3 & 286.7 & 4,300 \\
\hline Ful, Chemicals \& Disposal:............................ . & 220.0 & 220.0 & 220.0 & 240.0 & 1,200 \\
\hline Surface Maintenance............................................ . & 214.8 & 214.8 & 211.1 & 233.3 & 6,300 \\
\hline Subsurface Maintenance.................................. . & 155.6 & 166.7 & 166.7 & 177.8 & 1,600 \\
\hline \multirow[t]{2}{*}{ Total or Index } & 216.1 & 221.4 & 221.4 & 239.3 & 13,400 \\
\hline & \multicolumn{5}{|c|}{250 Thousand Cubic Feet Per Day } \\
\hline Direct Labor \& Overhead.................................... . & 253.3 & 266.7 & 273.3 & 286.7 & 4,300 \\
\hline Fuel, Chemicals \& Disposal............................. . & 225.0 & 225.0 & 225.0 & 240.0 & 4,800 \\
\hline Surface Maintenance.......................................... . & 212.2 & 212.2 & 212.2 & 230.6 & 11,300 \\
\hline Subsurface Maintenance.................................... . & 155.6 & 166.7 & 166.7 & 177.8 & 1,600 \\
\hline \multirow[t]{2}{*}{ Total or Index } & 216.1 & 219.4 & 220.4 & 236.6 & 22,000 \\
\hline & \multicolumn{5}{|c|}{500 Thousand Cublc Feet Per Day } \\
\hline Direct Labor \& Overhead................................... . & 253.3 & 266.7 & 273.3 & 286.7 & 4,300 \\
\hline Fuel, Chemicals \& Disposal.......................... . & 236.0 & 232.0 & 244.0 & 264.0 & 6,600 \\
\hline Surface Maintenance........................................ . & 195.7 & 195.7 & 195.7 & 213.0 & 9,800 \\
\hline Subsurface Maintenance................................. . & 155.6 & 166.7 & 166.7 & 177.8 & 1,600 \\
\hline Total or Index ${ }^{4+\ldots}$ & 211.6 & 213.7 & 217.9 & 234.7 & 22,300 \\
\hline
\end{tabular}


Table M7. Direct Annual Operating Costs and Indices for Gas Production in the Rocky Mountains (1 Well Producing from 8,000 Feet)

\begin{tabular}{|c|c|c|c|c|c|}
\hline \multirow{2}{*}{ Component } & \multicolumn{4}{|c|}{ Index $(1976=100)$} & \multirow{2}{*}{$\begin{array}{c}1997^{*} \\
\text { Cost } \\
\text { (dollars) }\end{array}$} \\
\hline & 1994 & 1995 & 1996 & 1997 & \\
\hline & \multicolumn{5}{|c|}{250 Thousand Cubic Feet Per Day } \\
\hline Direct Labor \& Overhead..................................... . & 258.8 & 270.6 & 276.5 & 294.1 & 5,000 \\
\hline Fuel, Chemicals \& Disposal............................ . & 231.7 & 229.3 & 236.6 & 251.2 & 10,300 \\
\hline Surface Maintenance................................... & 201.9 & 201.9 & 201.9 & 220.8 & 11,700 \\
\hline Subsurface Maintenance.................................. . & 164.3 & 164.3 & 171.4 & 178.6 & 2,500 \\
\hline \multirow[t]{2}{*}{ Total or Index $x^{\star \star} \ldots$} & 215.2 & 216.0 & 220.0 & 236.0 & 29,500 \\
\hline & \multicolumn{5}{|c|}{500 Thousand Cubic Feet Per Day } \\
\hline Direct Labor \& Overhead................................... . & 258.8 & 270.6 & 276.5 & 294.1 & 5,000 \\
\hline Fuel, Chemicals \& Disposal........................... . & 235.7 & 232.1 & 246.4 & 267.9 & 7,500 \\
\hline 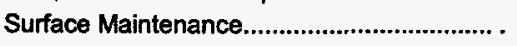 & 200.0 & 202.2 & 202.2 & 219.6 & 10,100 \\
\hline Subsurface Maintenance.................................. . & 164.3 & 164.3 & 171.4 & 178.6 & 2,500 \\
\hline Total or Index $x^{* \star}$ & 214.3 & 216.2 & 221.9 & 239.0 & 25,100 \\
\hline
\end{tabular}

Table M8. Direct Annual Operating Costs and Indices for Gas Production in the Rocky Mountains (1 Well Producing from 12,000 Feet)

\begin{tabular}{|c|c|c|c|c|c|}
\hline \multirow{2}{*}{ Component } & \multicolumn{4}{|c|}{ Index $(1976=100)$} & \multirow{2}{*}{$\begin{array}{c}1997^{\star} \\
\text { Cost } \\
\text { (dollars) }\end{array}$} \\
\hline & 1994 & 1995 & 1996 & 1997 & \\
\hline & \multicolumn{5}{|c|}{250 Thousand Cubic Feet Per Day } \\
\hline Direct Labor \& Overhead................................ . & 284.2 & 294.7 & 300.0 & 321.1 & 6,100 \\
\hline Fuel, Chemicals \& Disposal........................ & 231.4 & 229.4 & 233.3 & 251.0 & 12,800 \\
\hline Surface Maintenance................................... & 209.3 & 211.1 & 211.1 & 229.6 & 12,400 \\
\hline Subsurface Maintenance............................... . & 162.1 & 165.5 & 169.0 & 175.9 & 5,100 \\
\hline \multirow[t]{2}{*}{ Total or Index } & 217.0 & 219.0 & 221.6 & 237.9 & 36,400 \\
\hline & \multicolumn{5}{|c|}{500 Thousand Cubic Feet Per Day } \\
\hline Direct Labor \& Overhead................................ . & 284.2 & 294.7 & 300.0 & 321.1 & 6,100 \\
\hline Fuel, Chemicals \& Disposal......................... & 231.3 & 225.0 & 237.5 & 256.3 & 8,200 \\
\hline Surface Maintenance.................................... . & 210.6 & 212.8 & 212.8 & 231.9 & 10,900 \\
\hline Subsurface Maintenance.............................. . & 162.1 & 165.5 & 169.0 & 175.9 & 5,100 \\
\hline \multirow[t]{2}{*}{ Total or Index } & 215.7 & 217.3 & 222.0 & 238.6 & 30,300 \\
\hline & \multicolumn{5}{|c|}{1 Million Cubic Feet Per Day } \\
\hline Direct Labor \& Overhead............................... . & 284.2 & 294.7 & 300.0 & 321.1 & 6,100 \\
\hline Fuel, Chemicals \& Disposal.......................... & 234.4 & 231.1 & 241.0 & 260.7 & 15,900 \\
\hline Surface Maintenance.................................... . & 210.6 & 212.8 & 212.8 & 231.9 & 10,900 \\
\hline Subsurface Maintenance............................... . & 162.1 & 165.5 & 169.0 & 175.9 & 5,100 \\
\hline Total or Index & 219.9 & 221.2 & 226.3 & 243.6 & 38,000 \\
\hline
\end{tabular}




\title{
Section III
}

\author{
Appendix $\mathbf{N}$ \\ Epuiping and Operating Cost Indices \\ and Other Economic Indicators
}





\section{Appendix N}

\section{Equipping and Operating Cost Indices and Other Economic Indicators}

Appendix $\mathrm{N}$ contains a general overview of oil and gas economics from 1976 through 1997.

Unweighted aggregates of equipping and operating costs from the summary tables were indexed, with 1976 as the base year. The Gross Domestic Product (GDP) Implicit Price Deflator was used to deflate these indices and the Producer Price Indices (PPI). Each deflated index would equal 100 if the change in cost matched the change in the GDP for that index. The results appear in Tables $\mathrm{N} 1$ and $\mathrm{N} 2$ and are illustrated in Figures N1 and N2.

Although the aggregate average costs may not be the average costs for all oil and gas wells in the United States, it is possible to make some meaningful observations.

The deflated indices for oil lease equipment peaked in 1981 at 122.8 and continued in a general decline to a low of 70.8 in 1994. Volatile tubing prices have been the largest part of the changing equipment costs for much of the time but are less so than non-tubing equipment costs in the period 1994-1997. Gas well equipment prices, as shown in Figure N1, changed less than oil equipment prices, but only slightly. Equipment price trends are upward from 1994 to 1997.

The deflated indices for operating costs for both oil and gas leases peaked in 1982 at 145.2 and 122.8 , respectively, and declined until 1992 for oil leases and 1996 for gas leases, followed by increases through 1997 . The decline was primarily a reflection of the decrease in drilling activity and workovers which caused service companies to cut prices and their own costs drastically to stay in business. During 1997 , both drilling and well service activity reached levels believed by some observers to be limited by either equipment or personnel. 
Table N1. Indices and Gross Domestic Product Deflated Indices of the Aggregate Average Equipping Costs for Oil and Gas Fields and the Producer Price Index (PPI) (Capital Equipment)

\begin{tabular}{|c|c|c|c|c|c|c|c|}
\hline \multirow[b]{2}{*}{ Year } & \multicolumn{4}{|c|}{ Indices } & \multicolumn{3}{|c|}{$\begin{array}{c}\text { Gross Domestic Product } \\
\text { Deflated Indices }\end{array}$} \\
\hline & OII & Gas & $\mathbf{P P |}^{\mathbf{a}}$ & Deflator & OII & Gas & PP I \\
\hline $\begin{array}{l}1976 \\
1977 \\
1978 \\
1979 \\
1980 \\
1981 \\
1982 \\
1983 \\
1984 \\
1985 \\
1986 \\
1987 \\
1988 \\
1989 \\
1990 \\
1991 \\
1992 \\
1993 \\
1994 \\
1995 \\
1996 \\
1997\end{array}$ & $\begin{array}{l}100.0 \\
110.2 \\
120.7 \\
133.0 \\
154.4 \\
181.8 \\
191.6 \\
170.2 \\
190.0 \\
165.4 \\
147.1 \\
170.9 \\
169.6 \\
178.0 \\
170.9 \\
169.6 \\
178.0 \\
169.9 \\
166.6 \\
177.1 \\
183.9 \\
197.7\end{array}$ & $\begin{array}{l}100.0 \\
116.1 \\
127.3 \\
142.2 \\
161.4 \\
176.7 \\
183.4 \\
168.9 \\
160.5 \\
159.3 \\
153.0 \\
162.4 \\
172.6 \\
176.1 \\
189.3 \\
192.3 \\
200.9 \\
206.4 \\
184.2 \\
190.2 \\
196.6 \\
206.0\end{array}$ & $\begin{array}{l}100.0 \\
106.4 \\
114.8 \\
124.8 \\
138.2 \\
152.3 \\
161.0 \\
165.5 \\
169.4 \\
173.1 \\
176.7 \\
179.9 \\
184.1 \\
191.3 \\
197.9 \\
204.0 \\
207.9 \\
211.6 \\
215.9 \\
220.1 \\
222.7 \\
222.5\end{array}$ & $\begin{array}{l}100.0 \\
106.4 \\
114.1 \\
123.9 \\
135.3 \\
148.0 \\
157.3 \\
164.0 \\
170.2 \\
176.0 \\
180.6 \\
186.2 \\
193.0 \\
201.0 \\
209.8 \\
218.1 \\
224.3 \\
230.1 \\
235.4 \\
241.7 \\
247.2 \\
252.3\end{array}$ & $\begin{array}{r}100.0 \\
103.6 \\
105.7 \\
107.4 \\
114.1 \\
122.8 \\
121.8 \\
103.8 \\
111.7 \\
94.0 \\
81.4 \\
91.8 \\
87.9 \\
88.5 \\
81.4 \\
77.8 \\
79.4 \\
73.8 \\
70.8 \\
73.3 \\
74.4 \\
78.3\end{array}$ & $\begin{array}{r}100.0 \\
109.1 \\
111.5 \\
114.8 \\
119.3 \\
119.4 \\
116.6 \\
103.0 \\
94.3 \\
90.5 \\
84.7 \\
87.2 \\
89.4 \\
87.6 \\
90.2 \\
88.2 \\
89.6 \\
89.7 \\
78.2 \\
78.7 \\
79.5 \\
81.6\end{array}$ & $\begin{array}{r}100.0 \\
100.0 \\
100.6 \\
100.7 \\
102.1 \\
102.9 \\
102.4 \\
100.9 \\
99.6 \\
98.3 \\
97.8 \\
96.6 \\
95.4 \\
95.2 \\
94.3 \\
93.5 \\
92.7 \\
92.0 \\
91.7 \\
91.1 \\
90.1 \\
88.2\end{array}$ \\
\hline
\end{tabular}

"Producer Price Index (Capital Equipm ent) obtained from the Bureau of Labor Statistics, U.S. Departm ent of Labor.

'Grass Domestic implicit Price Deflators were obtained from the Bureau of Economic Analysis, U.S. Department of Commerce. Notes: The aggregate average costs are the average of the costs from summary Tables 1 and 6 and do not represent the

average costs of all wells in the United States.

Source: Energy Inform ation Administration, Office of Oil and Gas.

Table N2. The Gross Domestic Product Implicit Price Deflatorand the Gross Domestic Product Deflated Indices of Operating Costs for Oil and $G$ as Fields

\begin{tabular}{|c|c|c|c|c|c|}
\hline \multirow[b]{2}{*}{ Yoar } & \multirow{2}{*}{$\begin{array}{l}\text { GDP Implicit } \\
\text { Price Deflatora }\end{array}$} & \multicolumn{2}{|c|}{ Indices } & \multicolumn{2}{|c|}{$\begin{array}{l}\text { Gross Domestic Product } \\
\text { Deflated Indices } \\
\end{array}$} \\
\hline & & Oil & Gas & 011 & Gas \\
\hline 1976 & 100.0 & 100.0 & 100.0 & 100.0 & 100.0 \\
\hline 1977 & 106.4 & 117.5 & 114.6 & 110.4 & 107.7 \\
\hline 1978 & 114.1 & 130.3 & 121.8 & 114.1 & 106.7 \\
\hline 1979 & 123.9 & 144.0 & 135.8 & 116.2 & 109.6 \\
\hline 1980 & 135.3 & 174.2 & 156.4 & 128.7 & 115.6 \\
\hline 1981 & 148.0 & 204.2 & 181.5 & 138.0 & 122.6 \\
\hline 1982 & 157.3 & 228.4 & 193.2 & 145.2 & 122.8 \\
\hline 1983 & 164.0 & 226.2 & 190.8 & 137.9 & 116.3 \\
\hline 1984 & 170.2 & 230.1 & 192.0 & 135.2 & 112.8 \\
\hline 1985 & 176.0 & 232.2 & 190.7 & 131.9 & 108.3 \\
\hline 1986 & 180.6 & 212.9 & 181.1 & 117.9 & 100.3 \\
\hline 1987 & 186.2 & 210.5 & 177.4 & 113.1 & 95.3 \\
\hline 1988 & 193.0 & 220.1 & 184.9 & 114.0 & 95.8 \\
\hline 1989 & 201.0 & 229.1 & 189.6 & 114.0 & 94.3 \\
\hline 1990 & 209.8 & 239.6 & 200.9 & 114.2 & 95.8 \\
\hline 1991 & 218.1 & 242.9 & 204.7 & 111.4 & 93.9 \\
\hline 1992 & 224.3 & 247.9 & 208.5 & 110.5 & 93.0 \\
\hline 1993 & 230.1 & 257.8 & 216.0 & 112.1 & 93.9 \\
\hline 1994 & 235.4 & 270.7 & 216.0 & 115.0 & 91.8 \\
\hline 1995 & 241.7 & 268.8 & 218.9 & 111.2 & 90.6 \\
\hline 1996 & 247.2 & 282.9 & 222.6 & 114.4 & 90.1 \\
\hline 1997 & 252.3 & 293.8 & 235.8 & 116.4 & 93.5 \\
\hline
\end{tabular}

"Gross Domestic Product Implicit Price Deflators were obtained from the Bureau of Economic Analysis.

U.S. Departm ent of Comm erce.

Notes: The aggregate average costs are the average of the costs from summary Tables 3 and 14

and do not represent the average costs of all wells in the United $S$ tates

Source: Energy Inform ation Adm in istration, Office of $O$ il and $G$ as 
Figure N1. Gross Domestic Product Deflated Producer Price Indices, and Oil and Gas Field Equiping Cost Indices

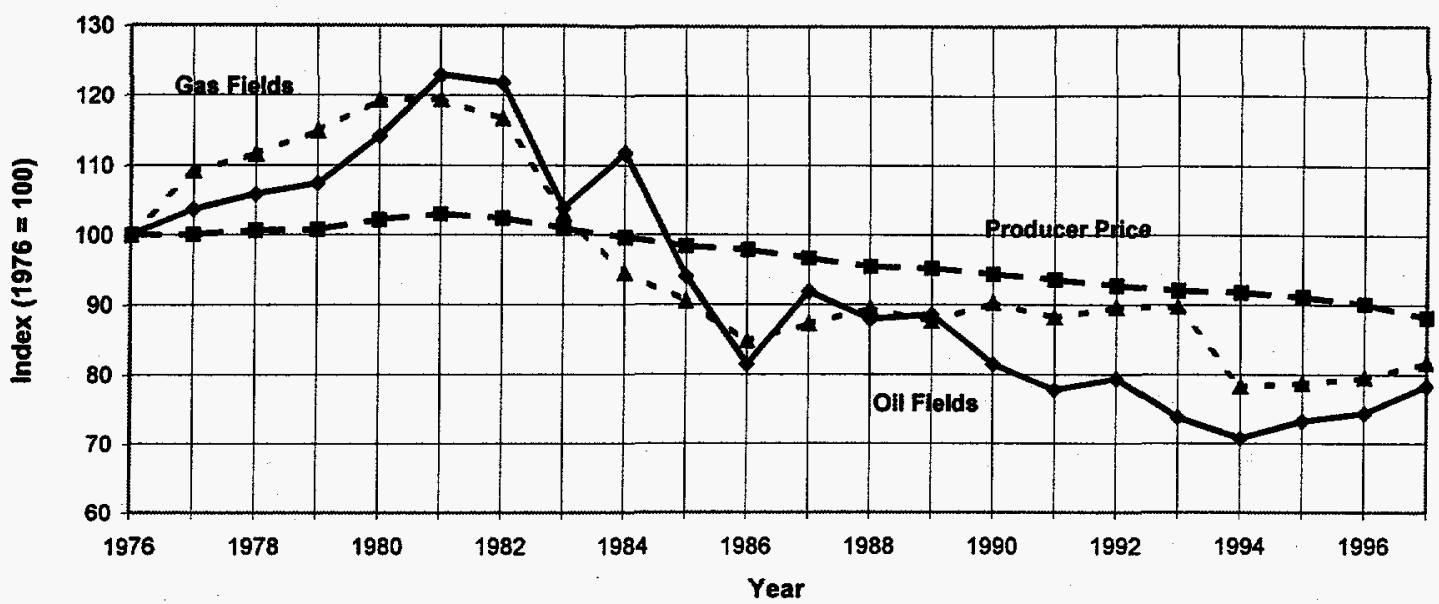

Source: Table N1.

Figure N2. Gross Domestic Product Deflated Operating Cost Indices for Oil and Gas Fields

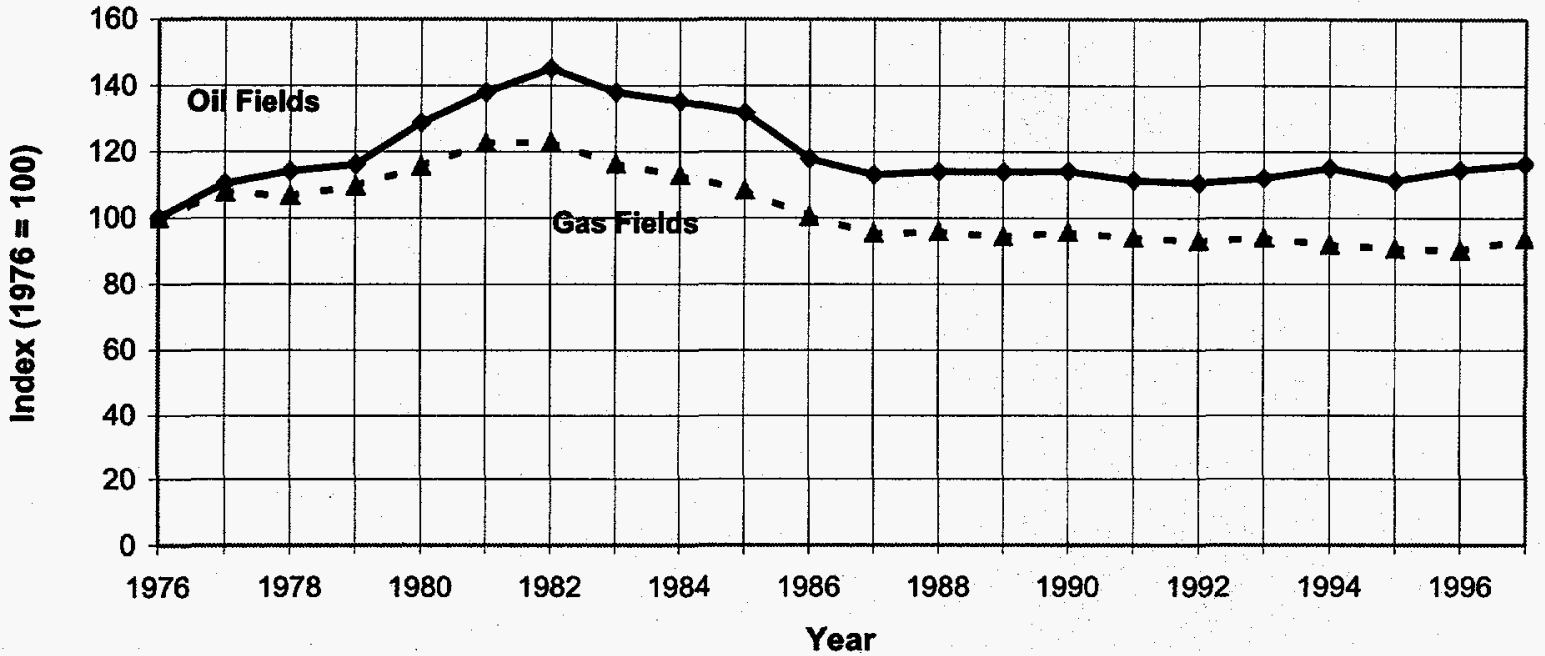

Source: Table N2. 

Glossary 



\section{Glossary}

Additional oil recovery: Recovery which follows primary, or natural depletion recovery, and is usually based on the application of processes which involve capital expenditures.

ad valorem: The basis for taxation of oil and gas operating properties, usually computed by expert assessment of current value.

API: American Petroleum Institute.

EIA: Energy Information Administration.

IPAA: Independent Petroleum Association of America.

JAS: Joint Association Survey, a survey of the cost of drilling wells in the U.S., conducted by the API, IPAA and MCOGA.

LACT: Lease automatic custody transfer, generally refers to unattended metering of oil sales from leases.

Mef: One thouand (standard) cubic feet.
MCOGA: Mid-Continent Oil and Gas Association, one of a number of regional associations of independent oil and gas operators.

Natural depletion: Means of recovering oil or gas relying on natural pressure in the reservoir rocks to expel substances to surface facilities for treatment and sale.

Secondary recovery: See additional recovery. One common type is by means of water injection (waterflood).

Stripper well: A well that produces $90 \mathrm{Mcf}$ per day or less of gas-well gas for a period of three consecutive months while producing at its maximum rate of flow or an oil well which produces less than 15 barrels of oil per day at its maximum rate of production for a period of three consecutive months.

WSU: Well service unit. Equipment used to maintain oil and gas wells. Usually mounted on vehicles for movement over roads. 